\title{
A Series of Crystallographically Characterized Linear and Branched $\sigma$-Alkane Complexes of Rhodium: From Propane to 3-Methylpentane
}

\author{
Alexander J. Bukvic, ${ }^{a, b}$ Arron L. Burnage, ${ }^{c}$ Graham J. Tizzard, ${ }^{d}$ Antonio J. Martínez- \\ Martínez, ${ }^{b}$ Alasdair I. McKay, ${ }^{b}$ Nicholas H. Rees, ${ }^{b}$ Bengt E. Tegner, ${ }^{c}$ Tobias Krämer, $^{c}$ \\ Heather Fish, ${ }^{a}$ Mark Warren, ${ }^{e}$ Simon J. Coles, ${ }^{d}$ Stuart A. Macgregor ${ }^{c *}$ and \\ Andrew S. Weller. ${ }^{\text {a* }}$ \\ ${ }^{a}$ Department of Chemistry, University of York, Heslington, York, YO10 5DD, UK. \\ Email: andrew.weller@york.ac.uk \\ ${ }^{\mathrm{b}}$ Department of Chemistry, Chemistry Research Laboratories, University of Oxford, \\ Oxford, OX1 3TA, UK. \\ ${ }^{\mathrm{c}}$ Institute of Chemical Sciences, Heriot-Watt University, Edinburgh, EH14 4AS, UK. \\ Email: S.A.Macgregor@hw.ac.uk
}

${ }^{\mathrm{d}}$ UK National Crystallography Service, University of Southampton, Highfield, Southampton, SO17 1BJ, UK.

e Diamond Light Source Ltd., Diamond House, Harwell Science and Innovation Campus, Didcot, OX11 0DE, UK. 


\section{TABLE OF CONTENTS}

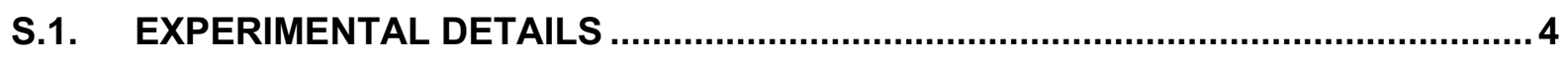

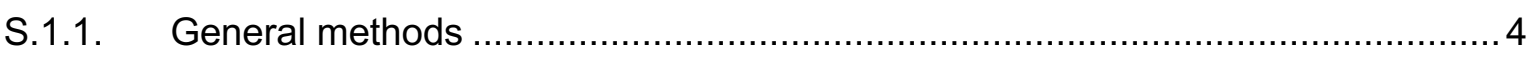

S.2. SYNTHETIC PROCEDURES .......................................................................... 6

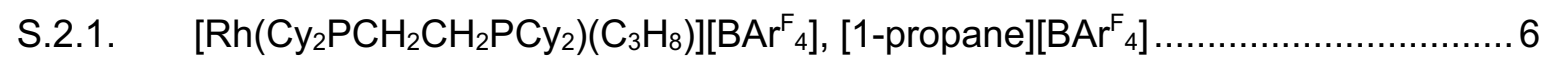

S.2.1.1. Synthesis of a precursor propene complex, [1-propene][BAr$\left.{ }_{4}{ }_{4}\right] \ldots \ldots \ldots \ldots \ldots . . . . .6$

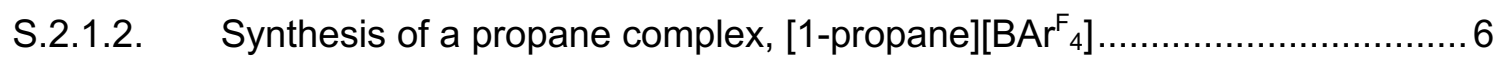

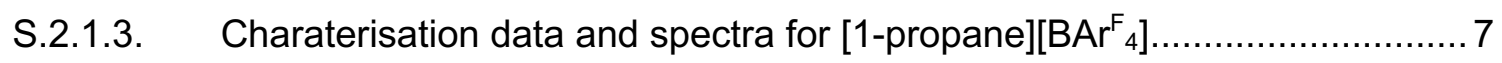

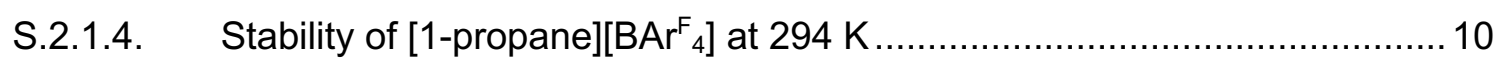

S.2.2. $\left[\mathrm{Rh}\left(\mathrm{Cy}_{2} \mathrm{PCH}_{2} \mathrm{CH}_{2} \mathrm{PCy}_{2}\right)\left(\mathrm{C}_{5} \mathrm{H}_{12}\right)\right]\left[\mathrm{BAr}{ }_{4}\right],[$ [1-(2-methylbutane $\left.)\right]\left[\mathrm{BAr} \mathrm{F}_{4}\right] \ldots \ldots \ldots \ldots \ldots . . . . . .11$

S.2.2.1. Synthesis of a precursor 2-methylbutane complex, [1-isoprene][BAr $\left.{ }_{4}\right] \ldots 11$

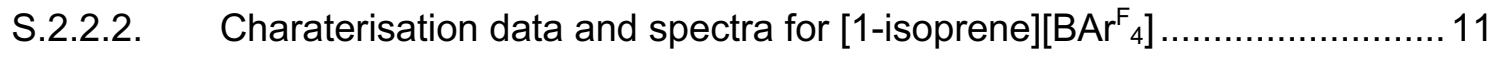

S.2.2.3. Synthesis of a 2-methylbutane complex, [1-(2-methylbutane)][BAr $\left.{ }_{4}\right] \ldots . . .16$

S.2.2.4. Charaterisation data and spectra for [1-(2-methylbutane $)]\left[B A r^{F}{ }_{4}\right] \ldots \ldots \ldots \ldots . . . .16$

S.2.3. $\left[\mathrm{Rh}\left(\mathrm{Cy}_{2} \mathrm{PCH}_{2} \mathrm{CH}_{2} \mathrm{PCy}_{2}\right)\left(\mathrm{C}_{5} \mathrm{H}_{10}\right)\right]\left[\mathrm{BAr}_{4}\right]$, [1-methylbutenes] $\left[\mathrm{BAr}{ }_{4}\right] \ldots \ldots \ldots \ldots \ldots . . . . . . .18$

S.2.3.1. Synthesis of a methylbutenes complex, [1-methylbutenes][BAr$\left.{ }_{4}\right] \ldots \ldots \ldots . . .18$

S.2.3.2. Charaterisation data and spectra for [1-methylbutenes] $\left[\mathrm{BAr} \mathrm{F}_{4}\right] \ldots \ldots \ldots \ldots \ldots$

S.2.3.3. Liberation of bound methylbutenes from [1-methylbutenes] $\left[\mathrm{BAr}_{4}{ }_{4}\right] \ldots \ldots \ldots .26$

S.2.3.4. Reaction kinetics of the dehydrogenation of [1-(2-methylbutane)][BArF$\left.{ }_{4}\right] .27$

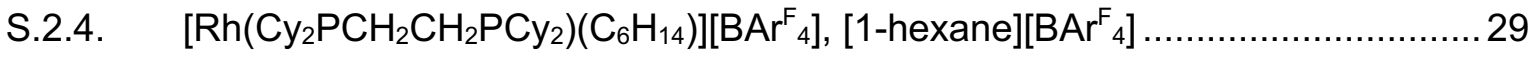

S.2.4.1. Synthesis of a precursor hexane complex, [1-hexadiene][BAr$\left.{ }_{4}\right] \ldots \ldots \ldots \ldots .29$

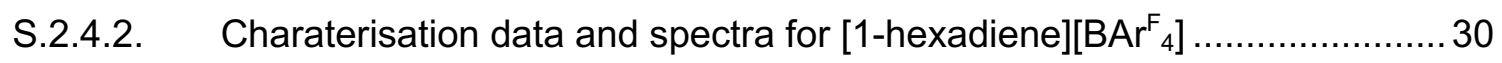

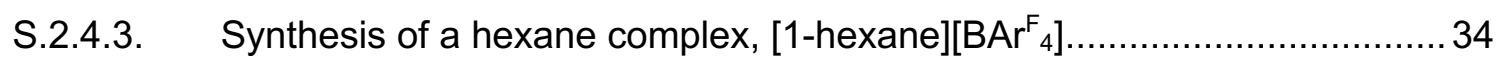

S.2.4.4. Charaterisation data and spectra for [1-hexane][BAr$\left.{ }_{4}\right] \ldots \ldots \ldots \ldots \ldots \ldots \ldots . . . . . . . . . . . .34$

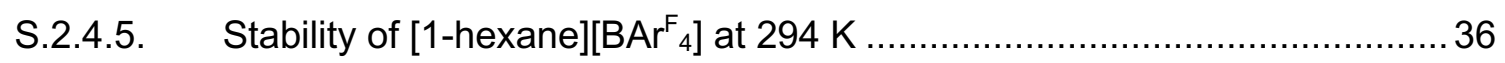

S.2.5. $\left[\mathrm{Rh}\left(\mathrm{Cy}_{2} \mathrm{PCH}_{2} \mathrm{CH}_{2} \mathrm{PCy}_{2}\right)\left(\mathrm{C}_{6} \mathrm{H}_{14}\right)\right]\left[\mathrm{BAr}_{4}{ }_{4}\right],[$ 1-(3-methypentane $\left.)\right]\left[\mathrm{BAr}_{4}{ }_{4}\right] \ldots \ldots \ldots \ldots . . . . .37$

S.2.5.1. Synthesis of a precursor 3-methylpentane complex, [1-( $\left.\left(\mathrm{C}_{6} \mathrm{H}_{10}\right)\right]\left[\mathrm{BAr}_{4}\right]$

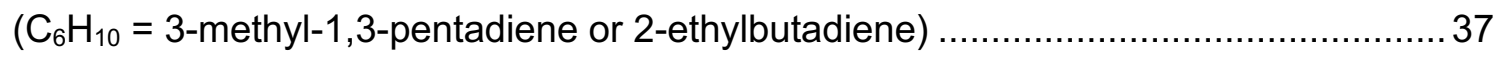

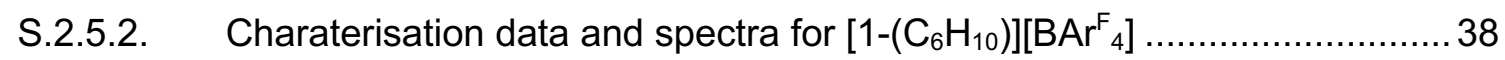

S.2.5.3. Liberation of bound $\mathrm{C}_{6}$-isomers from [1-( $\left.\left.\mathrm{C}_{6} \mathrm{H}_{10}\right)\right]\left[\mathrm{BAr}_{4}{ }_{4}\right] \quad\left(\mathrm{C}_{6} \mathrm{H}_{10}=\right.$ 3-methyl-1,3-pentadiene or 2-ethylbutadiene) ....................................................... 42

S.2.5.4. Synthesis of a 3-methylpentane complex, [1-(3-methylpentane)][BAr $\left.{ }_{4}\right]$... 44

S.2.5.5. Charaterisation data and spectra for [1-(3-methylpentane)][BArF $\left.{ }_{4}\right] \ldots \ldots \ldots . . . .44$

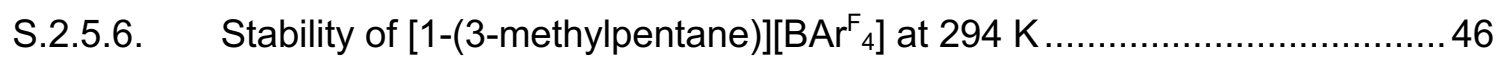

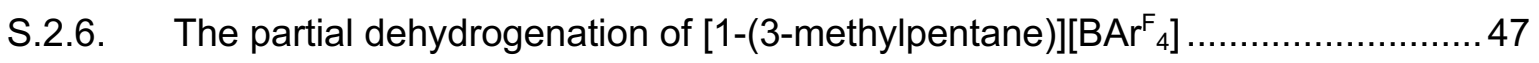

S.2.6.1. Proposed methylpentenes complex.................................................. 47 
S.2.6.2. Charaterisation data and spectra ....................................................... 48

S.2.6.3. Liberation of bound methylpentenes ................................................... 50

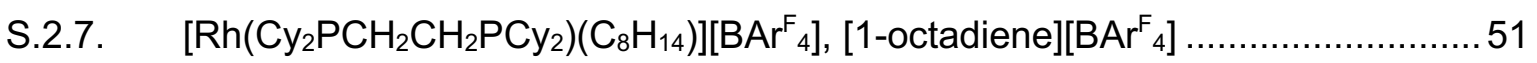

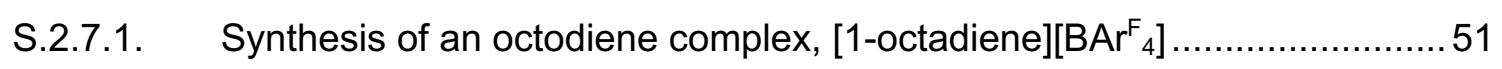

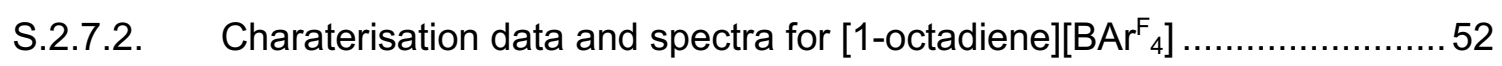

S.2.7.3. Attempted synthesis of ethane and octane complexes..............................54

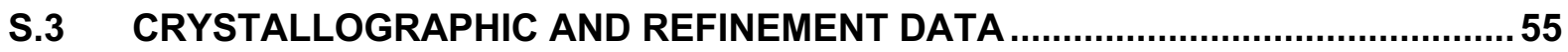

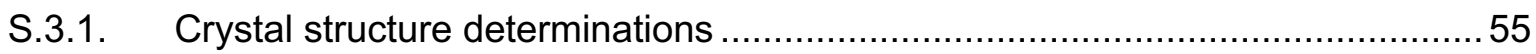

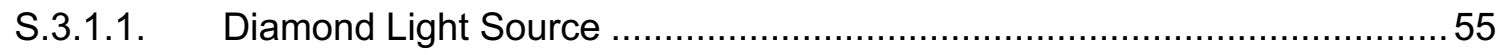

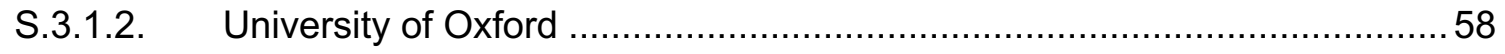

S.3.1.3. National Crystallography Service, Southampton, UK .............................58

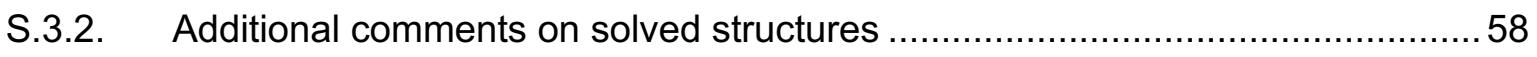

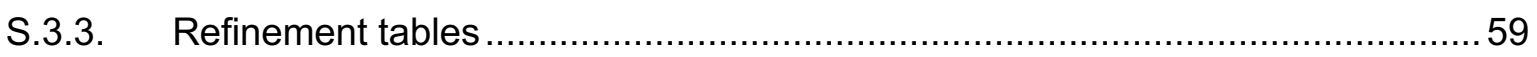

S.3.4. Structural images from single crystal X-ray diffraction experiments ..................62 62

S.3.5. Comparison of $\mathrm{Rh} \cdots \mathrm{C}$ distances from selected $\sigma$-alkane complexes................71

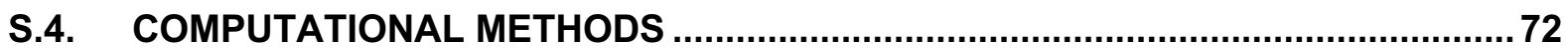

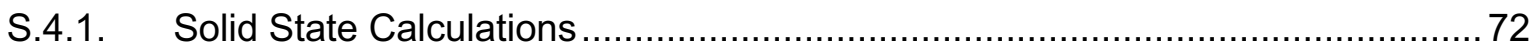

S.4.2. Molecular Calculations …..................................................................... 73

S.5. ELECTRONIC STRUCTURE ANALYSIS ..................................................... 76

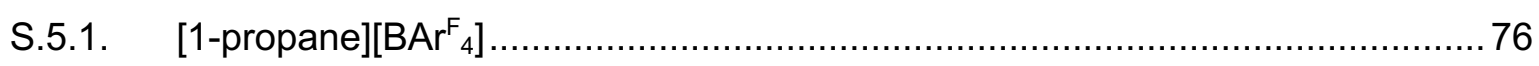

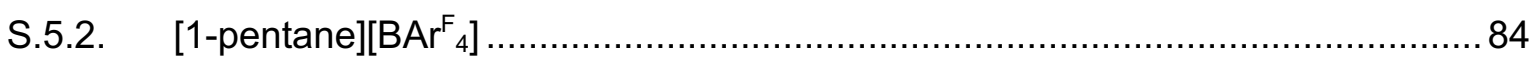

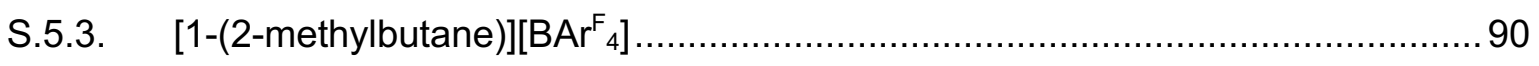

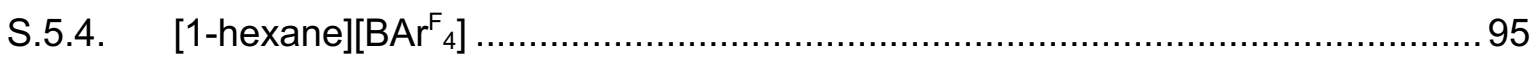

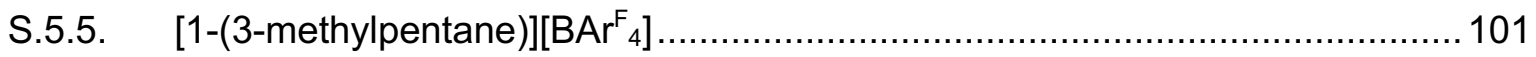

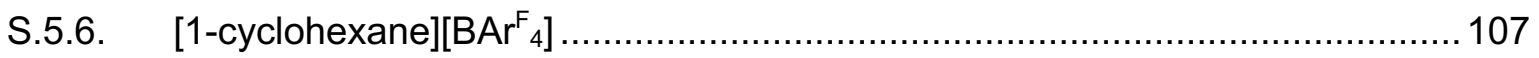

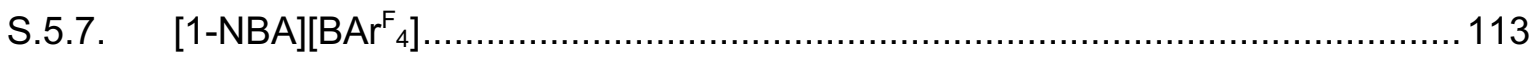

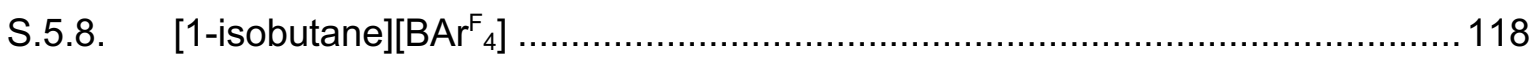

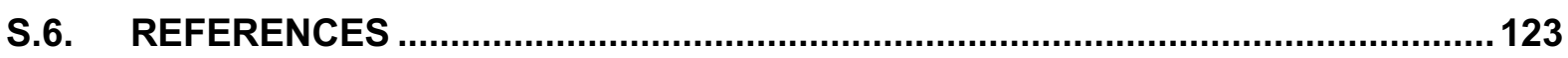




\section{S.1. Experimental details}

\section{S.1.1. General methods}

All manipulations (unless otherwise stated) were performed under an atmosphere of argon, using standard Schlenk techniques on a dual vacuum/inlet manifold or by employment of an MBraun glovebox. Glassware was dried in an oven at $130{ }^{\circ} \mathrm{C}$ overnight prior to use. Pentane, benzene and dichloromethane (abbreviated as $\mathrm{CH}_{2} \mathrm{Cl}_{2}$ ) were dried using an MBraun SPS-800 solvent purification system and degassed by three freeze-pump-thaw cycles. 1,2-difluorobenzene (abbreviated as $\mathrm{F}_{2} \mathrm{C}_{6} \mathrm{H}_{4}$ ) and 1,2-dichloroethene were prepared by stirring over $\mathrm{Al}_{2} \mathrm{O}_{3}$ for two hours then over $\mathrm{CaH}_{2}$ overnight before being vacuum distilled and subsequently degassed by three freeze-pump-thaw cycles. Dichloromethane- $d_{2}$ (abbreviated to $\mathrm{CD}_{2} \mathrm{Cl}_{2}$ ), acetonitrile- $d_{3}$ (abbreviated to MeCN- $d_{3}$ ) and acetone- $d_{6}$ were dried by stirring over $\mathrm{CaH}_{2}$ overnight before being vacuum distilled onto $3 \AA$ molecular sieves and subsequently degassed by three freeze-pump-thaw cycles. Benzene- $d_{6}$ (abbreviated as $\mathrm{C}_{6} \mathrm{D}_{6}$ ) was stirred over Na pieces overnight before being vacuum distilled onto $3 \AA$ molecular sieves and subsequently degassed by three freeze-pump-thaw cycles. Propene gas was purchased from CK Gases and used as received. All other chemicals were purchased from commercial vendors and used as received.

$\left[\mathrm{Rh}\left(\mathrm{Cy}_{2} \mathrm{PCH}_{2} \mathrm{CH}_{2} \mathrm{PCy}\right)\left(1,2-\mathrm{F}_{2} \mathrm{C}_{6} \mathrm{H}_{4}\right)\right]\left[\mathrm{BAr}_{4}\right]$

$\left[1-\mathrm{F}_{2} \mathrm{C}_{6} \mathrm{H}_{4}\right]\left[\mathrm{BAr}_{4}\right]^{\mathrm{S} 1}$, $\left[\mathrm{Rh}\left(\mathrm{Cy}_{2} \mathrm{PCH}_{2} \mathrm{CH}_{2} \mathrm{PCy}\right)(\mathrm{NBD})\right]\left[\mathrm{BAr}{ }_{4}\right] \quad(\mathrm{NBD}=$ norbornadiene $)$ [1-NBD][BArF $\left.{ }_{4}\right]^{\mathrm{S} 2}$ and $\left[\mathrm{Rh}\left(\mathrm{Cy}_{2} \mathrm{PCH}_{2} \mathrm{CH}_{2} \mathrm{PCy}_{2}\right)(\mathrm{NBA})\right]\left[\mathrm{BAr}_{4}{ }_{4}\right](\mathrm{NBA}=$ norbornane $)[1-\mathrm{NBA}]\left[\mathrm{BAr}_{4}\right]^{\mathrm{S2}}$ were prepared by the literature procedures.

Solution NMR data were collected on either a Bruker AVD $500 \mathrm{MHz}$ or a Bruker Ascend 400 $\mathrm{MHz}$ spectrometer at room temperature unless otherwise started. Non-deuterated solvents were locked to standard external $\mathrm{CD}_{2} \mathrm{Cl}_{2}$ solutions. Residual protio solvent resonances were used as a reference for ${ }^{1} \mathrm{H}$ NMR spectra. ${ }^{31} \mathrm{P}\left\{{ }^{1} \mathrm{H}\right\}$ NMR spectra were referenced externally to $85 \% \mathrm{H}_{3} \mathrm{PO}_{4}\left(\mathrm{D}_{2} \mathrm{O}\right)$. All chemical shifts $(\delta)$ are quoted in ppm and coupling constants in $\mathrm{Hz}$.

Solid state NMR (SSNMR) samples were prepared packing powdered microcrystalline sample into either a $3.2 \mathrm{~mm}$ or $4 \mathrm{~mm}$ zirconia solid state rotor inside an argon filled glove box. SSNMR spectra were obtained on a Bruker AVANCE III HD spectrometer equipped with a 9.4 Tesla magnet operating at $100.6 \mathrm{MHz}$ for ${ }^{13} \mathrm{C}$ and $162 \mathrm{MHz}$ for ${ }^{31} \mathrm{P}$ respectively, with a magic-angle spinning (MAS) rate of $10 \mathrm{kHz}$. Relaxation time for ${ }^{1} \mathrm{H}$ and contact time for ${ }^{31} \mathrm{P}\left\{{ }^{1} \mathrm{H}\right\}$ CP/MAS and ${ }^{13} \mathrm{C}\left\{{ }^{1} \mathrm{H}\right\}$ CP/MAS NMR experiments were optimised for each compound as appropriate. All ${ }^{13} \mathrm{C}\left\{{ }^{1} \mathrm{H}\right\} \mathrm{CP} / \mathrm{MAS}$ spectra were referenced to adamantane (up 
field methine resonance, $\delta 29.5)^{\mathrm{S} 3}$ on a scale where $\delta(T M S)=0$ as a secondary reference. The temperature for Variable Temperature (VT) NMR experiments at low temperatures was externally calibrated using lead nitrate $\left(\mathrm{PbNO}_{3}\right)$.

Electrospray ionization mass spectrometry (ESI-MS) was carried out using a Bruker MicrOTOF instrument directly connected to a modified Innovative Technology glovebox. ${ }^{\text {S4 }}$

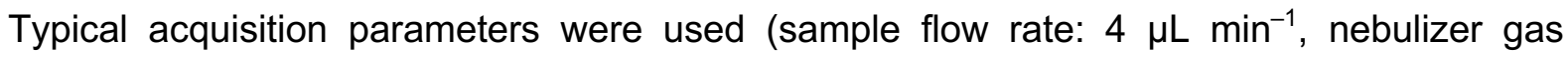
pressure: 0.4 bar, drying gas: Nitrogen at $333 \mathrm{~K}$ flowing at $4 \mathrm{~L} \mathrm{~min}{ }^{-1}$, capillary voltage: 4.5 $\mathrm{kV}$, exit voltage: $60 \mathrm{~V}$ ). The spectrometer was calibrated using a mixture of tetraalkyl ammonium bromides $\left[\mathrm{N}\left(\mathrm{C}_{\mathrm{n}} \mathrm{H}_{2 n+1}\right)_{4}\right] \mathrm{Br}(\mathrm{n}=2-8,12,16$ and 18). Samples were diluted to a concentration of $1 \times 10^{-6} \mathrm{M}$ in the appropriate solvent before sampling by ESI-MS.

Elemental analyses were conducted by Mr. Stephan Boyer at London Metropolitan University. 


\section{S.2. Synthetic Procedures}

\section{S.2.1. \\ $\left[\mathrm{Rh}\left(\mathrm{Cy}_{2} \mathrm{PCH}_{2} \mathrm{CH}_{2} \mathrm{PCy}_{2}\right)\left(\mathrm{C}_{3} \mathrm{H}_{8}\right)\right]\left[\mathrm{BAr}{ }_{4}\right]$, [1-propane][BArF $\left.{ }_{4}\right]$}

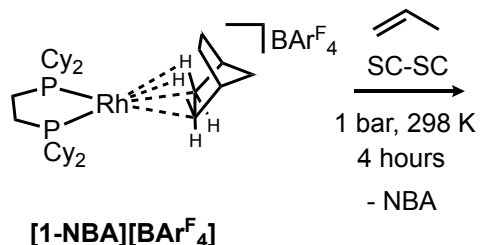

$[1-\mathrm{NBA}]\left[\mathrm{BArF}_{4}\right]$

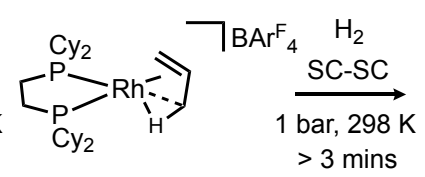

$\left[1-{\text { propene }]\left[B A r^{F}\right.}_{4}\right]$

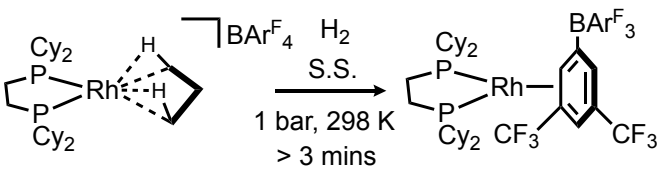

$[1-p r o p a n e]\left[\mathrm{BAr}_{4}{ }_{4}\right]$

$\left[1-\mathrm{BArF}_{4}\right]$

\section{S.2.1.1. Synthesis of a precursor propene complex, [1-propene][BAr $\left.{ }_{4}\right]$}

Note: [1-propene][BAr ${ }_{4}$ ] has been previously reported ${ }^{S 5}$ and the synthesis is repeated below. There has been no change in the synthetic route compared to that previously reported.

A J. Young flask ( $\sim 50 \mathrm{~mL}$ ) was loaded with crystals of [1-NBA][BAr ${ }_{4}$ ] (750 mgs), evacuated $\left(<2 \times 10^{-2} \mathrm{mbar}\right.$ ) and re-charged with propene gas (1 bar, $\left.298 \mathrm{~K}\right)$. The flask was left to stand for 4 hours. The excess propene and liberated norbornane could be removed under vacuum $\left(<2 \times 10^{-2}\right.$ mbar, 18 hours $)$ to quantitively yield $\left[\mathrm{Rh}\left(\mathrm{Cy}_{2} \mathrm{PCH}_{2} \mathrm{CH}_{2} \mathrm{PCy}_{2}\right)\left(\mathrm{C}_{3} \mathrm{H}_{6}\right)\right]\left[\mathrm{BAr}_{4}\right]$, [1-propene][BAr $\left.{ }_{4}{ }_{4}\right]$. The crystalline material is stored in an argon filled glove box in a freezer operating at $-25^{\circ} \mathrm{C}$.

\section{S.2.1.2. Synthesis of a propane complex, [1-propane][BAr ${ }_{4}$ ]}

For Single-Crystal X-Ray Diffraction: Conducted at Diamond Light Source synchrotron facility, ${ }^{\mathrm{S} 6}$ a single orange coloured crystal of [1-propene][BAr${ }_{4}{ }_{4}$ ] was affixed upon a $50 \mu \mathrm{m}$ MiTeGen Micromount using epoxy resin. The mount was inserted and affixed inside a 100 $\mu \mathrm{m}$ quartz capillary. This in turn was supported into a stainless-steel gas-line adapter fitted with Swagelok adapters, forming the gas cell. The above steps were performed inside an argon filled glove box. Each gas cell could be transferred and cycled onto the specially adapted gas-rig connected directly to the diffractometer upon Beamline I19 at Diamond Light Source. A unit-cell check was performed on each crystal to ensure the correct starting material had been mounted. Further details on this procedure and equipment is shown in Section S.3.1.1.

The crystal of [1-propene][BAr ${ }_{4}$ ] was then treated with $\mathrm{H}_{2}(1$ bar, $293 \mathrm{~K}$ ) as the cryostream was set to cool to $150 \mathrm{~K}$ at a rate of $360 \mathrm{~K} /$ hour. This gave an approximate time of $\sim 3$ minutes between $293 \mathrm{~K}$ and $275 \mathrm{~K}$. The colour of the crystals slowly turned from orange to 
dark red in this time and no reactivity with $\mathrm{H}_{2}$ was observed below this temperature. When the cryostream reached $150 \mathrm{~K}$, an X-ray diffraction study was undertaken and the structure of $\left[\mathrm{Rh}\left(\mathrm{Cy}_{2} \mathrm{PCH}_{2} \mathrm{CH}_{2} \mathrm{PCy}_{2}\right)\left(\mathrm{C}_{3} \mathrm{H}_{8}\right)\right]\left[\mathrm{BAr}_{4}\right]$ [1-propane][BAr $\left.{ }_{4}\right]$ was refined. Further details of this structural refinement are shown in Section S.3.

For Solid-State NMR Spectroscopy: A powdered microcrystalline sample of [1-propene][BAr ${ }_{4}$ ] (45 mgs) was packed in a $4.0 \mathrm{~mm}$ SSNMR rotor inside an argon filled glove box. The rotor was then placed in a custom-built glass $\mathrm{J}$. Young flask ${ }^{\mathrm{S5}}$ and the flask was placed under a vacuum $\left(<2 \times 10^{-2} \mathrm{mbar}\right)$ and then placed under an atmosphere of $\mathrm{H}_{2}(1$ bar, $298 \mathrm{~K}$ ). The rotor's cap was then immediately fitted under a flush of $\mathrm{H}_{2}$. The sample was rapidly transferred into the bore of a SSNMR spectrometer at $298 \mathrm{~K}$ and cooled to $158 \mathrm{~K}$ ( 10 minutes) before being analysed by ${ }^{31} \mathrm{P}\left\{{ }^{1} \mathrm{H}\right\}$ and ${ }^{13} \mathrm{C}\left\{{ }^{1} \mathrm{H}\right\}$ solid state NMR spectroscopy. At $158 \mathrm{~K}$ no reactivity with $\mathrm{H}_{2}$ is observed.

Note: This procedure was optimised. The time period at $298 \mathrm{~K}(\sim 10$ minutes) was found to be the experimentally shortest time possible, however this still lead to a significant amount of decomposition to $\left[\mathrm{Rh}\left(\mathrm{Cy}_{2} \mathrm{PCH}_{2} \mathrm{CH}_{2} \mathrm{PC} \mathrm{y}_{2}\right)\left\{\left(\eta^{6}-\mathrm{C}_{6} \mathrm{H}_{3}\left(\mathrm{CF}_{3}\right)_{2}\right) \mathrm{BAr}_{3}\right\}\right.$ ] [1-BAr ${ }_{4}$ ] $\left({ }^{31} \mathrm{P}\left\{{ }^{1} \mathrm{H}\right\} \mathrm{SSNMR}\right.$ : $\delta 91)^{\mathrm{S} 2}$ under an $\mathrm{H}_{2}$ atmosphere, see Figure $\mathrm{S} 2$. Minor signals assigned to [1-propene][BAr ${ }_{4}$ ] $\left({ }^{31} \mathrm{P}\left\{{ }^{1} \mathrm{H}\right\} \text { SSNMR: } \delta 99,93\right)^{\mathrm{S} 5}$ are also observed as a likely result of incomplete hydrogenation of the sample.

\section{S.2.1.3. Charaterisation data and spectra for [1-propane][BAr ${ }_{4}$ ]}

${ }^{31} \mathrm{P}\left\{{ }^{1} \mathrm{H}\right\}$ SSNMR (162 MHz, $158 \mathrm{~K}, 10 \mathrm{kHz}$ spin rate): $\delta 106.8$ (br s) ( 30 \% yield).

${ }^{13} \mathrm{C}\left\{{ }^{1} \mathrm{H}\right\}$ SSNMR (101 MHz, $158 \mathrm{~K}, 10 \mathrm{kHz}$ spin rate): $\delta 163.4$ (ArC), 133.8 (ArC), 129.3 (ArC), 124.5 (ArC), 116.5 (ArC), 34.6, 29.2, 25.1, 19.2, 15.5 (multiple aliphatic resonances). 


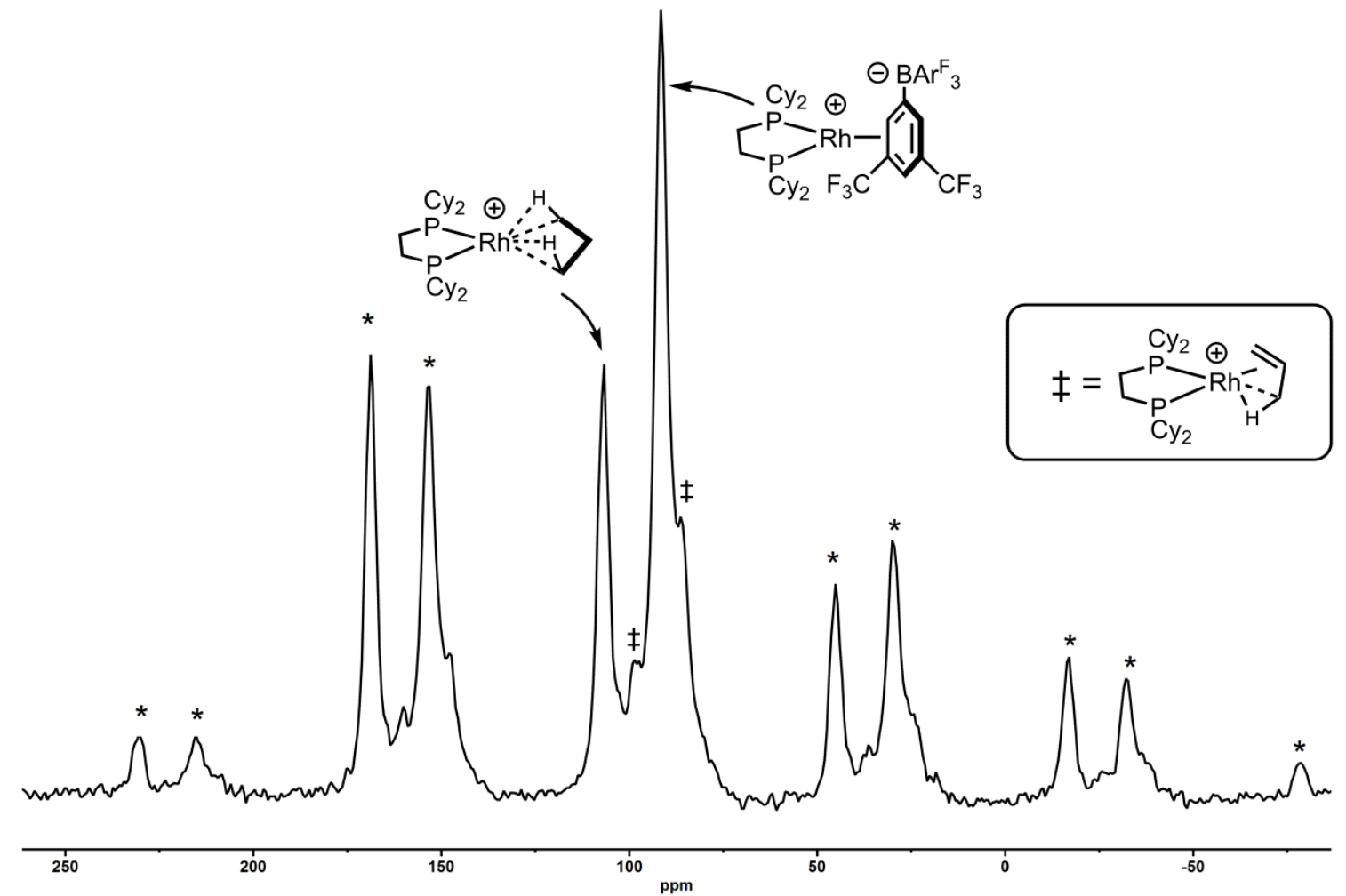

Figure S1: The ${ }^{31} \mathrm{P}\left\{{ }^{1} \mathrm{H}\right\}$ SSNMR spectrum (162 MHz, $158 \mathrm{~K}, 10 \mathrm{kHz}$ spin rate) of [1-propane][BAr ${ }_{4}$ ] prepared in situ after 3 minutes under $\mathrm{H}_{2}$ at $298 \mathrm{~K}$. The resonances marked * are due to spinning sidebands and $\ddagger$ from [1-propene][BAr ${ }_{4}{ }^{\mathrm{S}}{ }^{\mathrm{S}}$

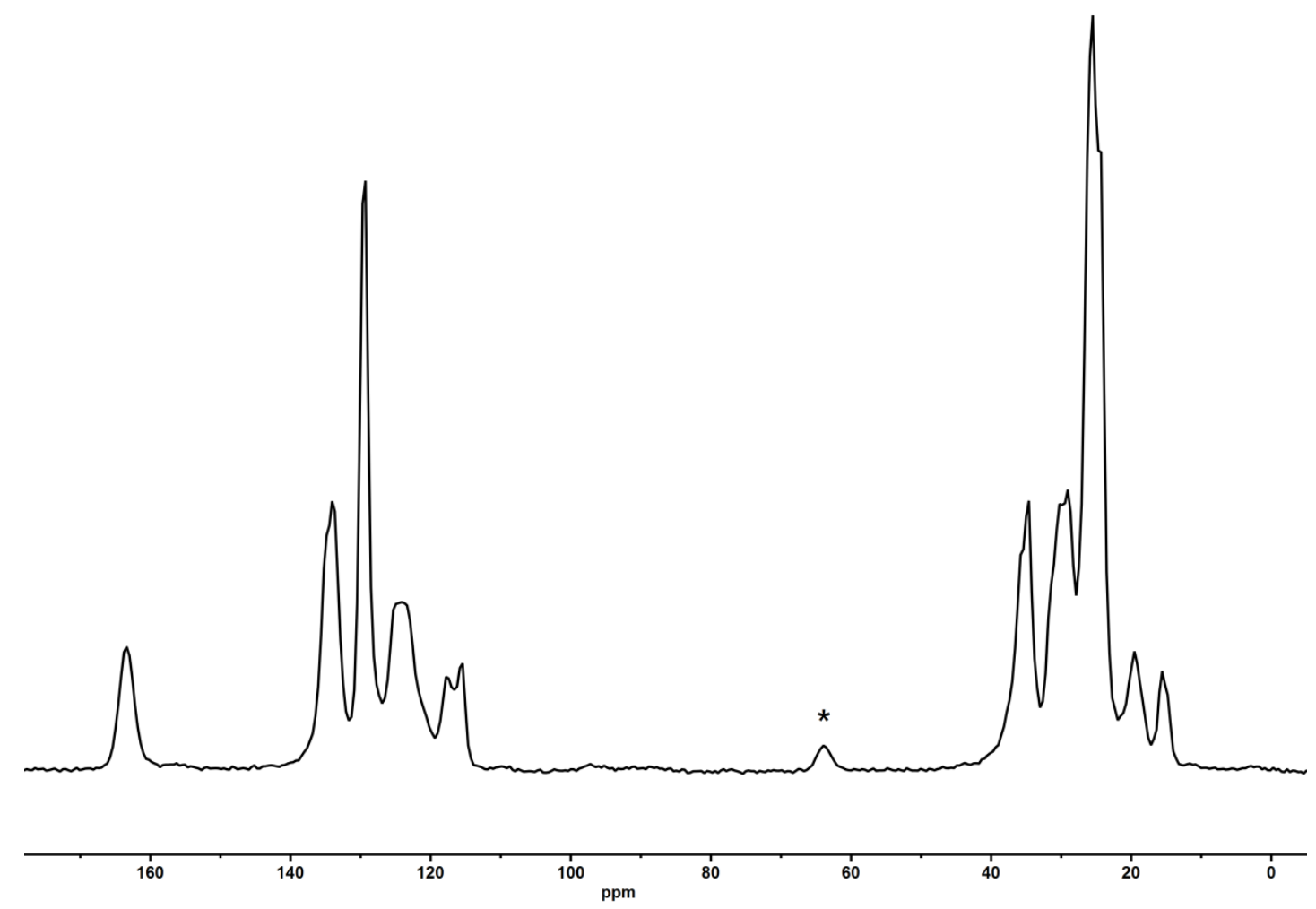

Figure S2: The ${ }^{13} \mathrm{C}\left\{{ }^{1} \mathrm{H}\right\}$ SSNMR spectrum (100 MHz, $158 \mathrm{~K}$, under $\mathrm{H}_{2}, 10 \mathrm{kHz}$ spin rate) of [1-propane][BAr ${ }_{4}$ ] prepared in situ. The resonance marked * is due to a spinning sideband. 
In Figure S1, we suggest that the multiple species present are due to the unique hydrogenation kinetics present during in situ hydrogenations within the solid-state rotor. This is not the case compared to in situ single crystal experiments, as only evidence for [1-propane][BAr ${ }_{4}$ ] could be refined. The densely packed nature of the material within the SSNMR rotor limits the movement of $\mathrm{H}_{2}$ throughout the rotor within the time and so, longer hydrogenation times than necessary are required to fully hydrogenate the sample. However, these longer hydrogenation times also lead to an increased concentration of decomposition product [1-BAr $\left.{ }_{4}\right]$.

The procedure has been optimised for [1-propane][BAr $\left.{ }_{4}\right]$ and the signals relating to [1-propene][BAr$\left.{ }_{4}\right]$ in Figure $\mathrm{S} 1$ are most likely from incomplete hydrogenation of the sample within this time and are from sample most likely located at the bottom of the rotor. This effect is not present in single-crystal X-ray diffraction studies as $\mathrm{H}_{2}$ gas can access the crystal from multiple points, visualised in Figure S3.

a

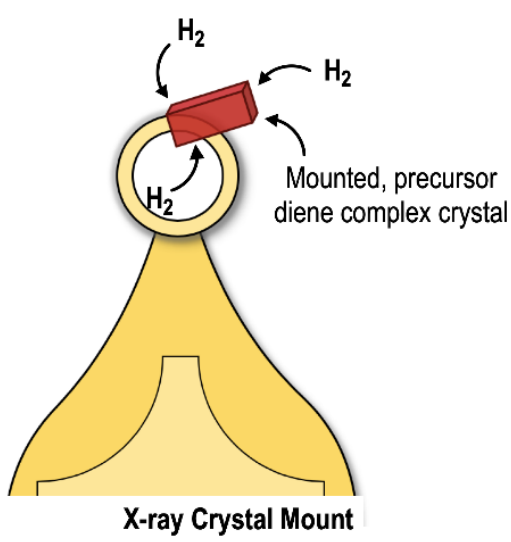

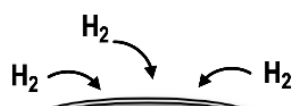

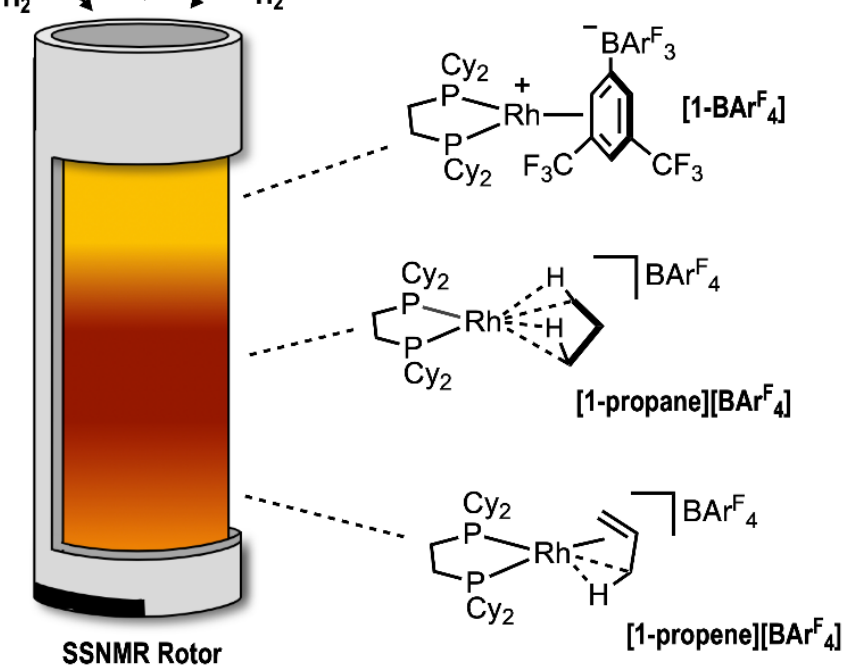

Figure S3: Hydrogen gas accessibility in a) single-crystal X-ray diffraction experiments and b) SSNMR spectroscopy. 


\section{S.2.1.4. Stability of [1-propane][BAr $\left.{ }_{4}\right]$ at $294 \mathrm{~K}$}

On the same sample as presented in Figures S1, the solid-state NMR spectrometer probe was warmed to $294 \mathrm{~K}$ (from $158 \mathrm{~K}$ ) and ${ }^{31} \mathrm{P}\left\{{ }^{1} \mathrm{H}\right\}$ SSNMR spectra collected until complete decomposition of [1-propane][BAr${ }_{4}$ ] was observed. During this time, the solid-state NMR rotor was under a flushing $\mathrm{N}_{2}$ atmosphere.

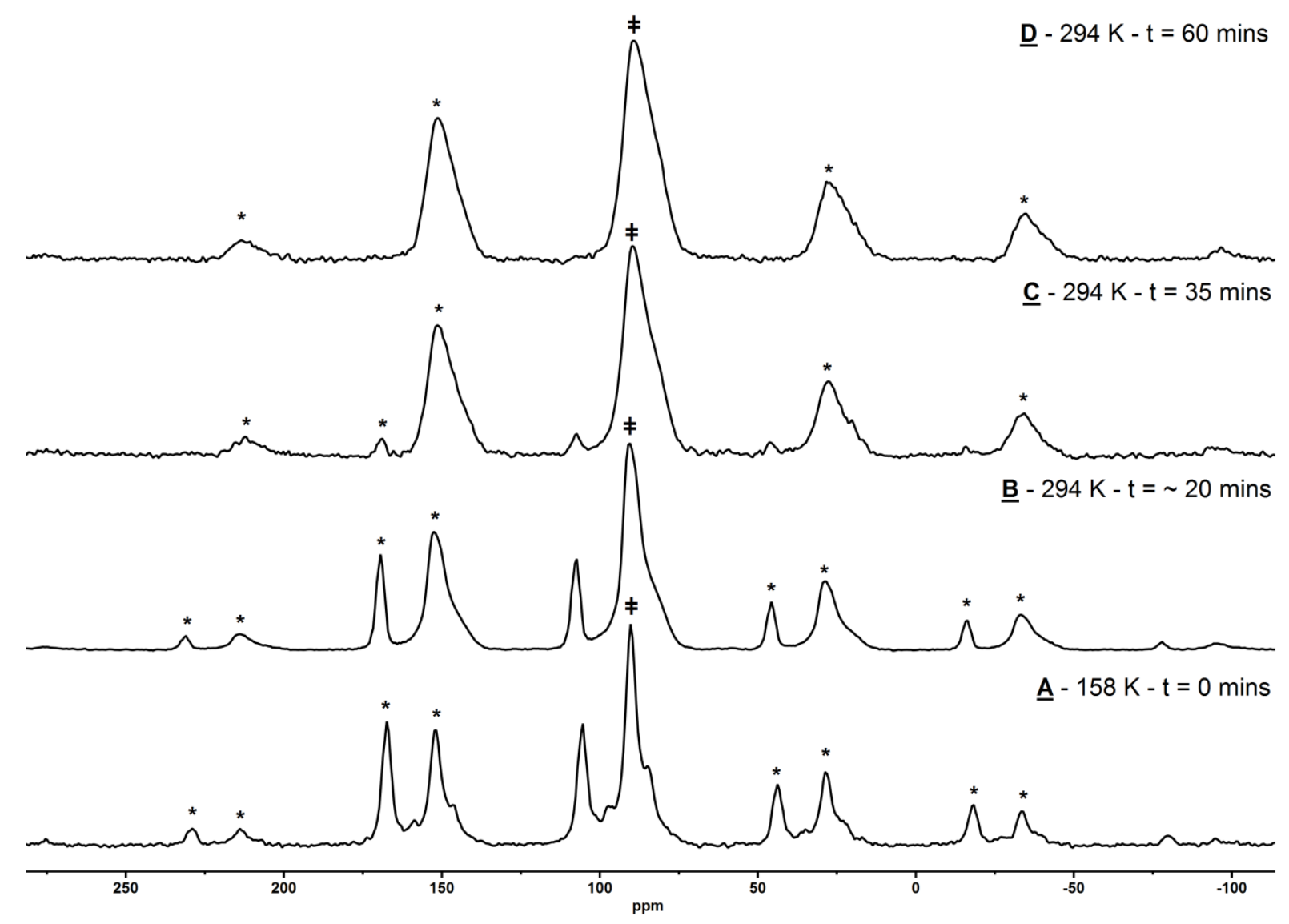

Figure S4: The ${ }^{31} \mathrm{P}\left\{{ }^{1} \mathrm{H}\right\}$ SSNMR spectra (162 $\mathrm{MHz}, 10 \mathrm{kHz}$ spin rate) of $\mathbf{A}$ : [1-propane][BAr ${ }_{4}$ ], as prepared at $158 \mathrm{~K}$. B: Spectrum collected upon warming to $294 \mathrm{~K}$. Total time at $294 \mathrm{~K}$ including instrument warming, stabilisation and experimental set-up / collection is $\mathbf{2 0}$ minutes. C: Total time at $294 \mathrm{~K}$ including instrument warming, stabilisation and experimental set-up / collection is 35 minutes. D: Total time at $294 \mathrm{~K}$ including instrument warming, stabilisation and experimental set-up / collection is $\mathbf{6 0}$ minutes. Peaks marked * are spinning sidebands and $\neq$ are assigned to $\left[1-\mathrm{BAr}_{4}{ }_{4}\right.$ in all parts. 


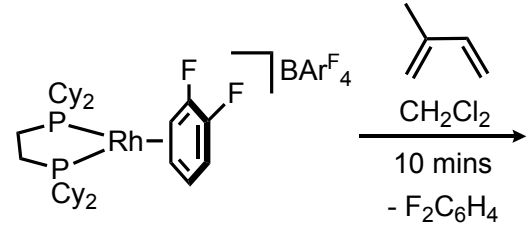

$\left[1-\mathrm{F}_{2} \mathrm{C}_{6} \mathrm{H}_{4}\right]\left[\mathrm{BAr}_{4}\right]$

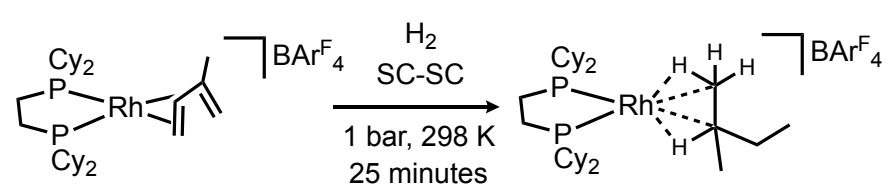

[1-isoprene][BArF$\left.{ }_{4}\right]$

[1-(2-methylbutane)][BArF$\left.{ }_{4}\right]$

\section{S.2.2.1. Synthesis of a precursor 2-methylbutane complex, [1-isoprene][BAr ${ }_{4}$ ]}

A pale-yellow solution of $\left[1-\mathrm{F}_{2} \mathrm{C}_{6} \mathrm{H}_{4}\right]\left[\mathrm{BAr}_{4}\right]$ (600 mgs, $\left.0.40 \mathrm{mmol}\right)$ in $\mathrm{CH}_{2} \mathrm{Cl}_{2}(20 \mathrm{~mL})$ was treated with neat 2-methyl-1,3-butadiene $(50 \mu \mathrm{L}, 0.5 \mathrm{mmol})$ at ambient temperature. The resultant deep red solution was stirred at ambient temperature for 30 minutes. Pentane (100 $\mathrm{mL}$ ) was then added with vigorous stirring which resulted in the formation of a red precipitate. The solid was isolated by filtration and washed with pentane $(2 \times 20 \mathrm{~mL})$. The solid was then dissolved in $\mathrm{CH}_{2} \mathrm{Cl}_{2}(\sim 8 \mathrm{~mL})$ and filtered into a J. Youngs crystallisation flask and layered with pentane. Deep red crystals were obtained after storage for 1 week at room temperature of $\left[\mathrm{Rh}\left(\mathrm{Cy}_{2} \mathrm{PCH}_{2} \mathrm{CH}_{2} \mathrm{PCy}_{2}\right)\left(\right.\right.$ iso- $\left.\left.\mathrm{C}_{5} \mathrm{H}_{8}\right)\right]\left[\mathrm{BAr}_{4}{ }_{4}\right]$, [1-isoprene][BAr ${ }_{4}$ ] (Yield: 551 mgs, $95 \%)$.

\section{S.2.2.2. Charaterisation data and spectra for [1-isoprene][BAr ${ }_{4}$ ]}

${ }^{1} \mathrm{H}$ NMR ( $\mathrm{CD}_{2} \mathrm{Cl}_{2}, 298 \mathrm{~K}, 400 \mathrm{MHz}$ ): $\delta 7.73$ (s, 8H, ortho-ArH), 7.57 (s, 4H, para-ArH), 5.16 (d. of d. of d., $1 \mathrm{H}$, alkene- $\mathrm{H}, J=13.8,8.1,1.6 \mathrm{~Hz}$ ), $4.38(\mathrm{~d}, 1 \mathrm{H}$, alkene- $\mathrm{H}, J=2.8 \mathrm{~Hz}), 4.32$ (d. of d. of d., $1 \mathrm{H}$, alkene- $\mathrm{H}, J=7.8,4.1,1.3 \mathrm{~Hz}$ ), 2.74 (d. of $\mathrm{m} ., 1 \mathrm{H}$, alkene- $\mathrm{H}, J=14.1,1.3$ $\mathrm{Hz}$ ), 2.70 (br. m., $1 \mathrm{H}$, alkene-H), 2.23-1.52 (br m, 31H, multiple overlapping aliphatic $\mathrm{CH}$ ), 1.42-0.84 (br m, 20H, multiple overlapping aliphatic $\mathrm{CH}$ ).

${ }^{31} \mathrm{P}\left\{{ }^{1} \mathrm{H}\right\}$ NMR $\left(\mathrm{CD}_{2} \mathrm{Cl}_{2}, 298 \mathrm{~K}, 162 \mathrm{MHz}\right): \delta 84.3\left(\mathrm{~d}, \mathrm{~J}_{\mathrm{RhP}}=163 \mathrm{~Hz}, J_{\mathrm{PP}}=22 \mathrm{~Hz}\right), \delta 79.5(\mathrm{~d}$, $J_{\mathrm{RhP}}=169 \mathrm{~Hz}, J_{\mathrm{PP}}=22 \mathrm{~Hz}$ ).

${ }^{19} \mathrm{~F}\left\{{ }^{1} \mathrm{H}\right\}$ NMR $\left(\mathrm{CD}_{2} \mathrm{Cl}_{2}, 298 \mathrm{~K}, 377 \mathrm{MHz}\right): \delta-62.9(\mathrm{~s})$.

${ }^{13} \mathrm{C}\left\{{ }^{1} \mathrm{H}\right\}$ NMR $\left(\mathrm{CD}_{2} \mathrm{Cl}_{2}, 298 \mathrm{~K}, 126 \mathrm{MHz}\right): \delta 162.4$ (q, J $\mathrm{J}_{\mathrm{B}}=50 \mathrm{~Hz}$, ipso-ArC), 135.1 (s, ortho$\operatorname{ArC}$ ), 129.3 (q, $J_{C F}=32 \mathrm{~Hz}$, meta-ArC), 125.1 (q, $J_{C F}=272 \mathrm{~Hz},-C_{3}$ ), 120.0 (apparent triplet, $\mathrm{C}=\mathrm{C}, \mathrm{J}=4.3 \mathrm{~Hz}$ ), 117.8 (s, para-ArC), 101.2 (apparent triplet, $\mathrm{C}=\mathrm{C}, \mathrm{J}=4.3 \mathrm{~Hz}$ ), 63.7 (d. of d. of d., $\mathrm{C}=\mathrm{C}, \mathrm{J}=14.4,5.3,1.5 \mathrm{~Hz}$ ), 62.8 (d. of d. of d., $\mathrm{C}=\mathrm{C}, J=10.9,7.6,1.7 \mathrm{~Hz}$ ), $38.4-22.6$ ( $\mathrm{m}$, overlapping cyclohexyl- $\mathrm{CH}_{2}$ ). 
The solid-state structure of [1-isoprene][BAr ${ }_{4}$ ] shows two crystallographically distinct orientations of the bound isopentadiene ligand (see Section S.3.). These two disorder components can be identified in the solid-state NMR spectra:

${ }^{31} \mathrm{P}\left\{{ }^{1} \mathrm{H}\right\}$ SSNMR (162 MHz, $294 \mathrm{~K}, 10 \mathrm{kHz}$ spin rate): Orientation 1: $\delta 86.2\left(\mathrm{~d}, \mathrm{~J}_{\mathrm{RhP}}=152\right.$ $\mathrm{Hz}), 75.5\left(\mathrm{~d}, J_{\mathrm{RhP}}=165 \mathrm{~Hz}\right)$. Orientation 2: $\delta 81.9\left(\mathrm{~d}, J_{\mathrm{RhP}}=147 \mathrm{~Hz}\right), 74.1\left(\mathrm{~d}, J_{\mathrm{RhP}}=150 \mathrm{~Hz}\right)$.

${ }^{13} \mathrm{C}\left\{{ }^{1} \mathrm{H}\right\}$ SSNMR (101 MHz, $294 \mathrm{~K}, 10 \mathrm{kHz}$ spin rate): $\delta 164.7$ (ipso-ArC), 134.5 (ortho-ArC), 129.7 (meta-ArC), 124.4 (br, - $\mathrm{CF}_{3}$ ), 120.5 (C=C), 117.3 (para-ArC), 116.1 (para-ArC), 104.3 $(C=C), 103.0(C=C), 96.4(C=C), 94.3(C=C), 91.6(C=C), 88.0(C=C), 60.4(C=C), 38.7$ 18.7 (m, overlapping cyclohexyl- $\mathrm{CH}_{2}$ ).

Note: Peaks that are assigned $(\mathrm{C}=\mathrm{C})$ as they disappear upon hydrogenation of the sample (see Figure S10).

ESI-MS (calc. for $\left.\left[\mathrm{Rh}\left(\mathrm{Cy}_{2} \mathrm{PCH}_{2} \mathrm{CH}_{2} \mathrm{PCy}_{2}\right)\left(\mathrm{C}_{5} \mathrm{H}_{8}\right)\right]^{+}\right): \mathrm{m} / \mathrm{z} 593.2912$ (593.2907).

Elemental Analysis (calc. for $\mathrm{C}_{63} \mathrm{H}_{68} \mathrm{BF}_{24} \mathrm{P}_{2} \mathrm{Rh}$ ): C 52.06 (51.94), H 4.50 (4.70). 


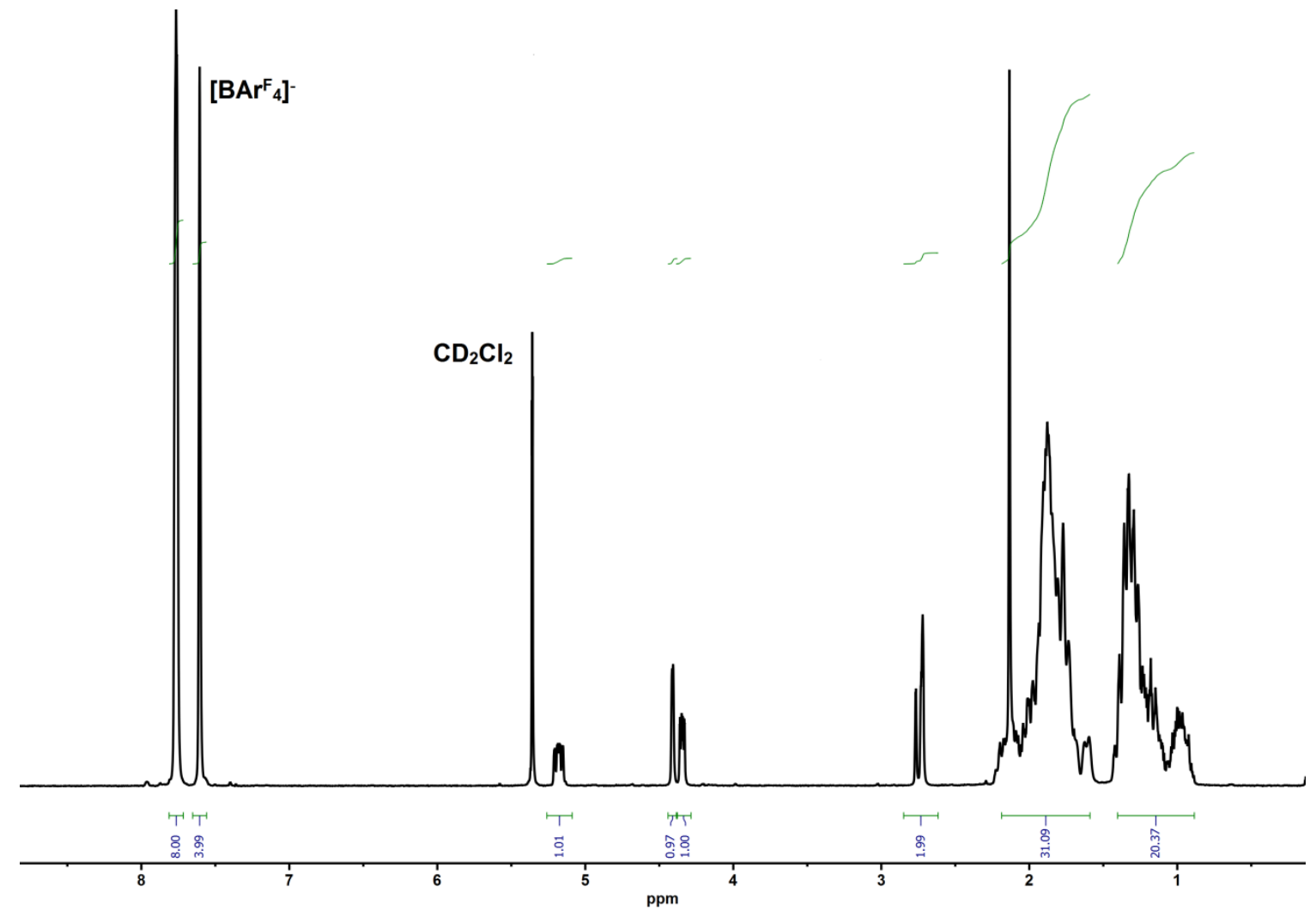

Figure S5: The solution ${ }^{1} \mathrm{H}$ NMR spectrum $\left(\mathrm{CD}_{2} \mathrm{Cl}_{2}, 298 \mathrm{~K}, 400 \mathrm{MHz}\right)$ of [1-isoprene][BArF$\left.{ }_{4}\right]$.

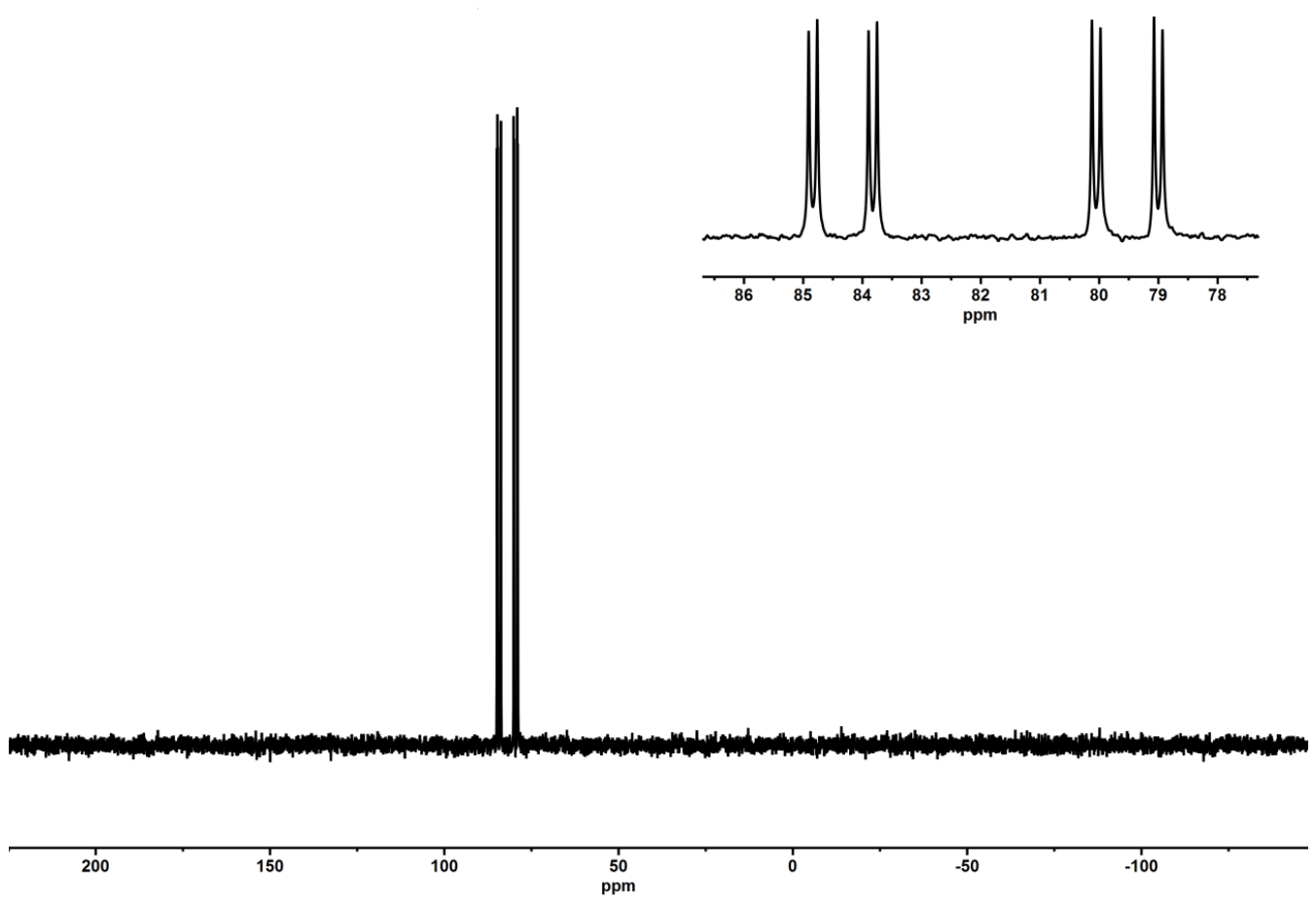

Figure S6: The solution ${ }^{31} \mathrm{P}\left\{{ }^{1} \mathrm{H}\right\}$ NMR spectrum $\left(\mathrm{CD}_{2} \mathrm{Cl}_{2}, 298 \mathrm{~K}, 162 \mathrm{MHz}\right)$ of [1-isoprene][BAr ${ }_{4}$ ]. The inset is an enlargement of the resonances between $\delta 85$ and 78 . 


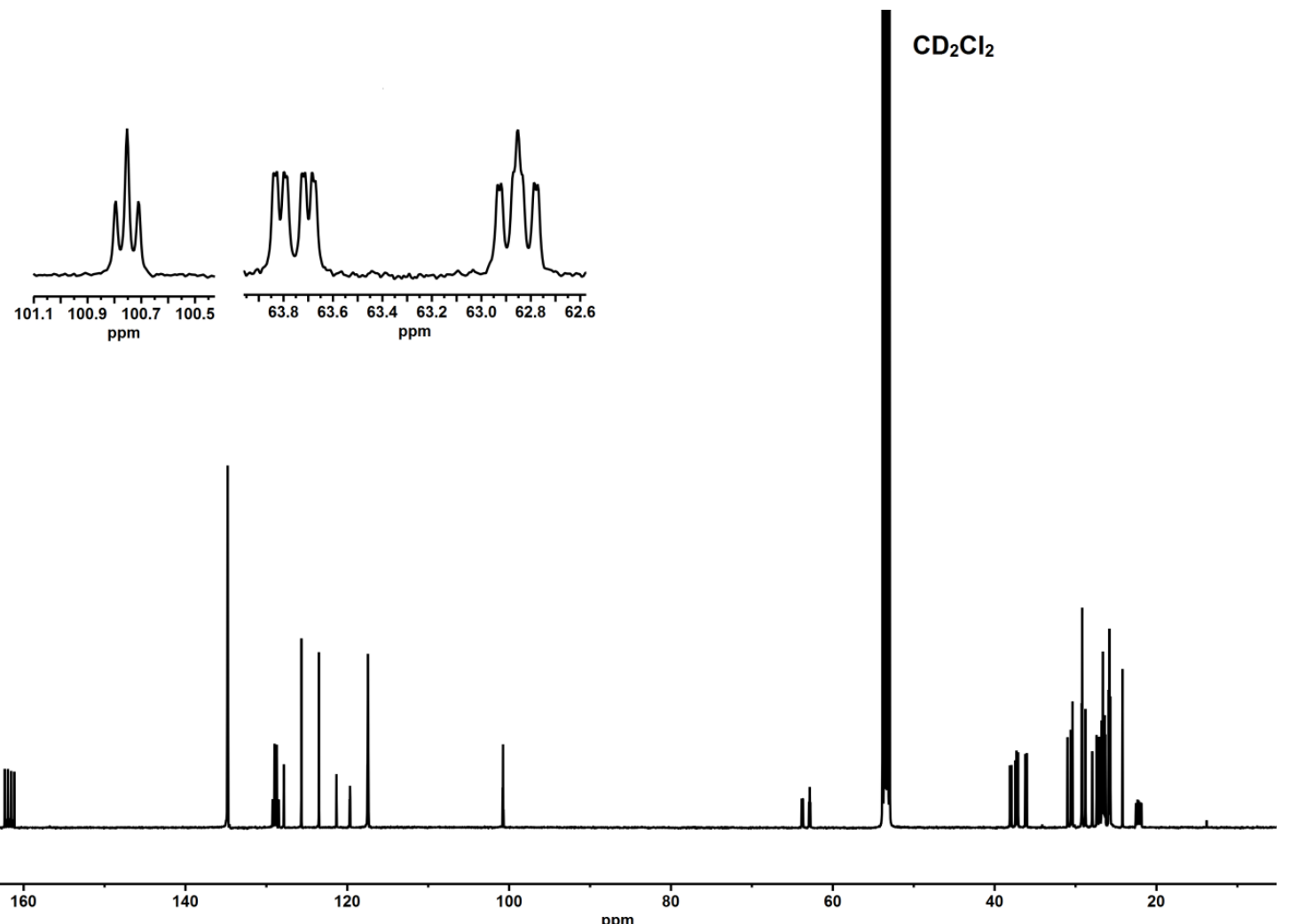

Figure S7: The solution ${ }^{13} \mathrm{C}\left\{{ }^{1} \mathrm{H}\right\}$ NMR ${ }^{\mathrm{ppm}}$ spectrum $\left(\mathrm{CD}_{2} \mathrm{Cl}_{2}, 298 \mathrm{~K}, 126 \mathrm{MHz}\right)$ of [1-isoprene][BAr${ }_{4}$ ]. The inset is an enlargement of the resonances between $\delta 101$ and 62 .

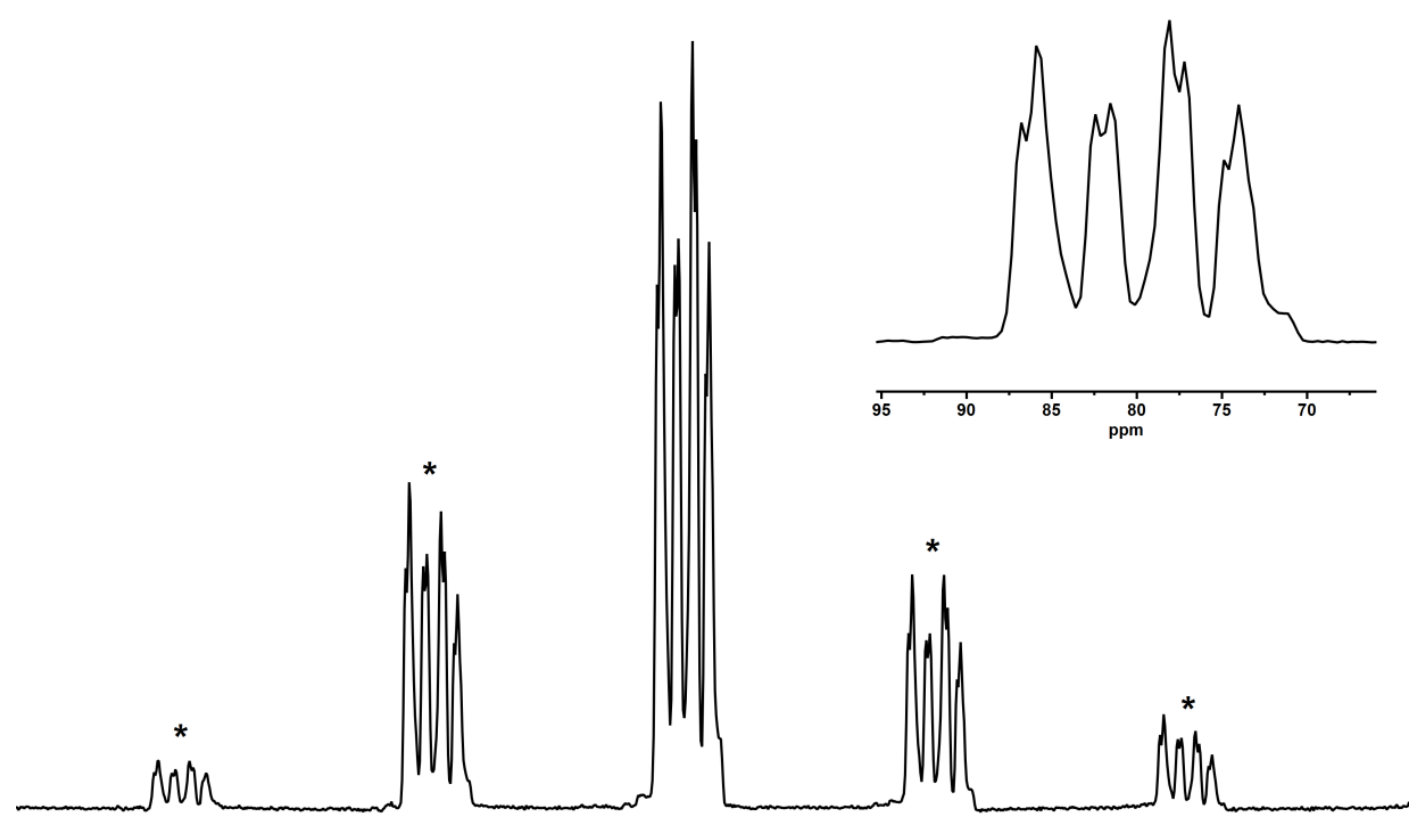

Figure S8: The ${ }^{31} \mathrm{P}\left\{{ }^{1} \mathrm{H}\right\}$ SSNMR spectrum ${ }^{150}(162 \mathrm{MHz}, 294 \mathrm{~K}, 10 \mathrm{kHz}$ spin rate) of [1-isoprene][BAr${ }_{4}^{\mathrm{F}}$ ]. The resonances marked * are due to spinning sidebands. The inset is an enlargement of the resonances between $\delta 101$ and 62 . 


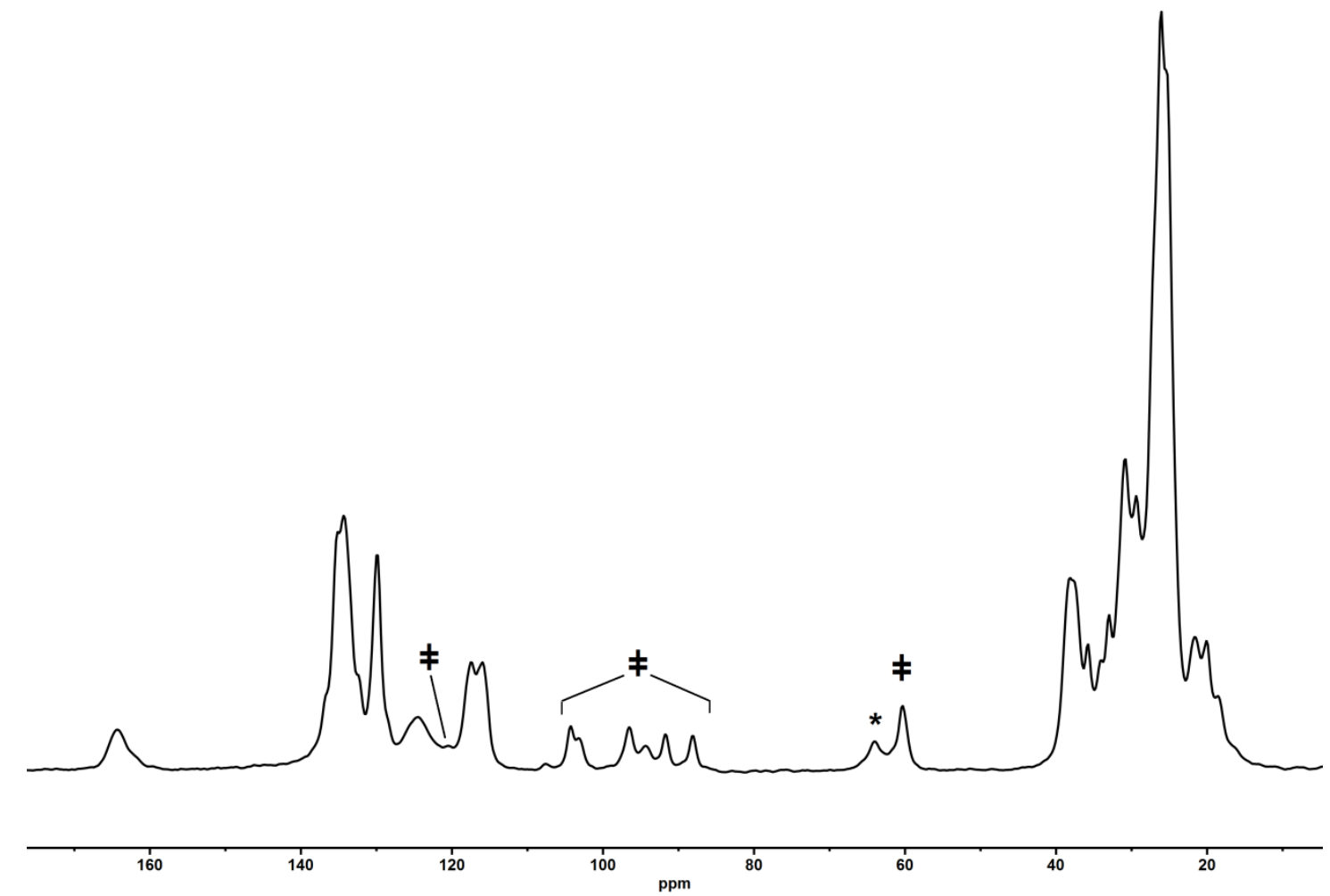

Figure S9: The ${ }^{13} \mathrm{C}\left\{{ }^{1} \mathrm{H}\right\}$ SSNMR spectrum (101 MHz, $298 \mathrm{~K}, 10 \mathrm{kHz}$ spin rate) of [1-isoprene][BAr$\left.{ }_{4}^{\mathrm{F}}\right]$. The resonances marked * are due to spinning sidebands. The alkene resonances have been assigned $\neq$. 


\section{S.2.2.3. Synthesis of a 2-methylbutane complex, [1-(2-methylbutane)][BAr $\left.{ }_{4}\right]$}

For Single-Crystal X-Ray Diffraction: Ruby red crystals of [1-isoprene][BAr ${ }_{4}{ }_{4}$ (1.5 mgs) $^{-}$ were placed in a J. Young NMR tube and treated with $\mathrm{H}_{2}$ (1 bar, $298 \mathrm{~K}, 25$ minutes). The colour of the crystals slowly turned a dark red over this time. The crystalline material was then removed from the tube and coated with Fomblin ${ }^{\circledR} Y$ oil under an argon flush. A suitable crystal was then selected and transferred to the cryostream of a diffractometer $(150 \mathrm{~K})$. A $X$-ray diffraction study was undertaken and the structure of $\left[\mathrm{Rh}\left(\mathrm{Cy}_{2} \mathrm{PCH}_{2} \mathrm{CH}_{2} \mathrm{PCy}\right)\left(\mathrm{C}_{5} \mathrm{H}_{12}\right)\right]\left[\mathrm{BAr}_{4}\right]$ [1-(2-methylbutane)][BAr$\left.{ }_{4}{ }_{4}\right]$ was refined. For further details of structural refinement please refer to Section S.3.

For Solid-State NMR Spectroscopy: A powdered microcrystalline sample of [1-isoprene][BAr ${ }_{4}$ ] (45 mgs) was packed in a $4.0 \mathrm{~mm}$ SSNMR rotor, inside an argon filled glove box. The rotor was then placed in a custom-built glass $\mathrm{J}$. Young flask ${ }^{\mathrm{S5}}$ and the sample was then exposed to $\mathrm{H}_{2}(1$ bar, $298 \mathrm{~K}$ ). After 20 minutes, the rotor cap was fitted under a flush of $\mathrm{H}_{2}$. The sample was rapidly transferred to the bore of a SSNMR spectrometer, cooled to $158 \mathrm{~K}\left(\sim 10\right.$ minutes) and analysed by ${ }^{31} \mathrm{P}\left\{{ }^{1} \mathrm{H}\right\}$ and ${ }^{13} \mathrm{C}\left\{{ }^{1} \mathrm{H}\right\}$ solid-state NMR spectroscopy.

Note: These conditions were optimised to form [1-(2-methylbutane)][BAr ${ }_{4}$ ]. At $158 \mathrm{~K}$, no onward reactivity with $\mathrm{H}_{2}$ is observed. When $\mathrm{H}_{2}$ is replaced by argon at $298 \mathrm{~K}$, as so the rotor is packed under an argon atmosphere, rapid dehydrogenation to form a mixture of [1-methylbutenes] [BAr ${ }_{4}$ ] isomers are observed before cooling to $158 \mathrm{~K}$, see Section S.2.4. Further exposure to $\mathrm{H}_{2}$ (90 minutes) at $298 \mathrm{~K}$ results in complete decomposition to $\left[1-\mathrm{BAr}_{4}{ }^{\mathrm{S}}{ }^{\mathrm{S} 2}\right.$

\section{S.2.2.4. Charaterisation data and spectra for [1-(2-methylbutane)][BAr $\left.{ }_{4}\right]$}

${ }^{31} \mathrm{P}\left\{{ }^{1} \mathrm{H}\right\}$ SSNMR (162 MHz, $158 \mathrm{~K}, 10 \mathrm{kHz}$ spin rate): $\delta 106.9\left(\mathrm{~d}, J_{\mathrm{RhP}}=204 \mathrm{~Hz}\right), 104.1$ (d, $J_{\mathrm{RhP}}=220 \mathrm{~Hz}$ ).

${ }^{13} \mathrm{C}\left\{{ }^{1} \mathrm{H}\right\}$ SSNMR (101 MHz, $158 \mathrm{~K}, 10 \mathrm{kHz}$ spin rate): $\delta 163.3$ (ipso-ArC), 134.1 (ortho-ArC), 128.9 (meta-ArC), 123.7 (br, - $\mathrm{CF}_{3}$ ), 116.6 (para-ArC), 39.9, 36.6, 34.8, 31.1, 30.1, 28.3, 25.0, 20.2, 17.3, 13.3, 10.1, 8.42 (multiple aliphatic resonances). 

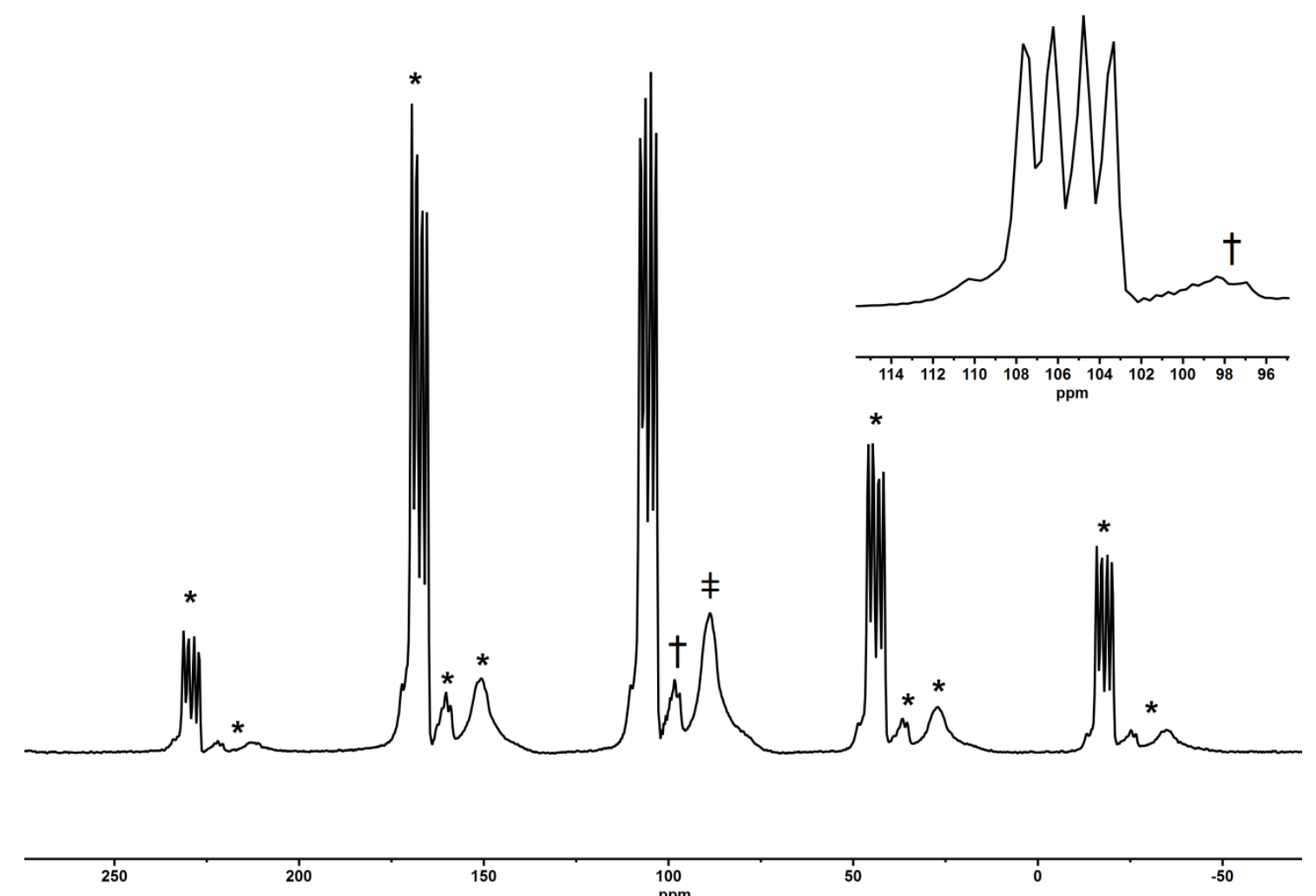

Figure S10: The ${ }^{31} \mathrm{P}\left\{{ }^{1} \mathrm{H}\right\}$ SSNMR spectrum (162 MHz, $158 \mathrm{~K}, 10 \mathrm{kHz}$ spin rate) of [1-(2-methylbutane)][BAr ${ }_{4}$ ]. Peaks marked * are spinning sidebands, $\neq$ are assigned to $\left[1-\mathrm{BAr}_{4}{ }_{4}^{\mathrm{S2}}\right.$ and $\dagger$ to [1-methylbutenes][BAr ${ }_{4}$ ]. The inset is an enlargement of the resonances between $\delta 114$ and 96 .

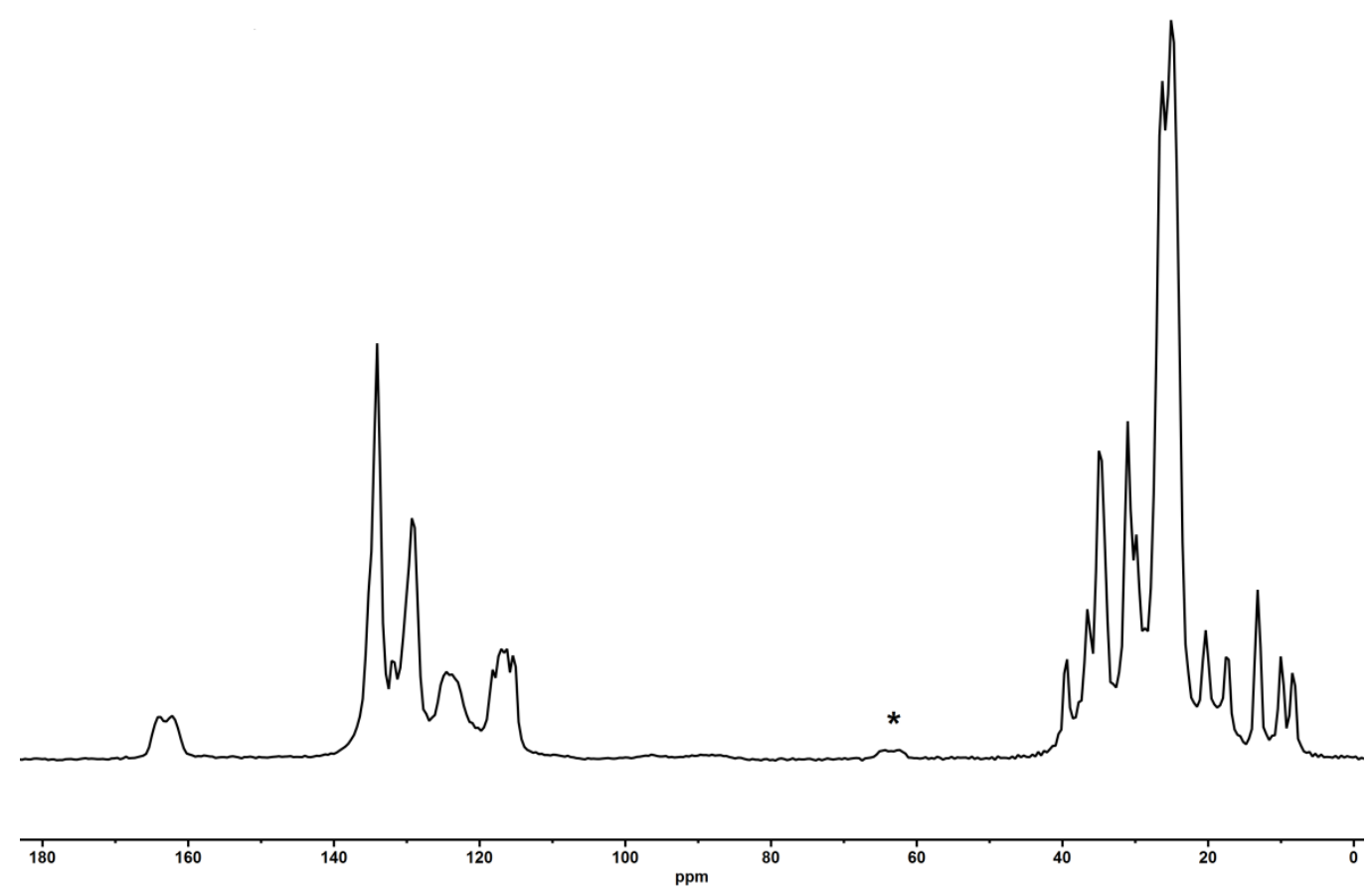

Figure S11: The ${ }^{13} \mathrm{C}\left\{{ }^{1} \mathrm{H}\right\}$ SSNMR spectrum (101 MHz, $158 \mathrm{~K}, 10 \mathrm{kHz}$ spin rate) of [1-(2-methylbutane)][BAr $\left.{ }_{4}\right]$. The resonances marked * are due to spinning sidebands. 
S.2.3. $\left[\mathrm{Rh}\left(\mathrm{Cy}_{2} \mathrm{PCH}_{2} \mathrm{CH}_{2} \mathrm{PCy}_{2}\right)\left(\mathrm{C}_{5} \mathrm{H}_{10}\right)\right]\left[\mathrm{BAr}_{4}^{\mathrm{F}}\right]$, [1-methylbutenes][BAr $\left.{ }_{4}\right]$

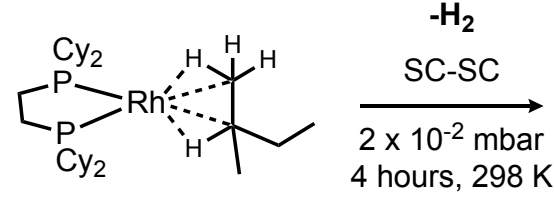

[1-(2-methylbutane)][BArF $\left.{ }_{4}\right]$

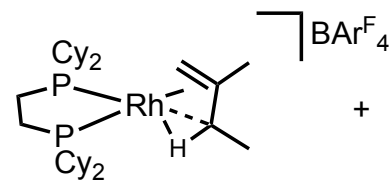

2-methylbut-1-ene

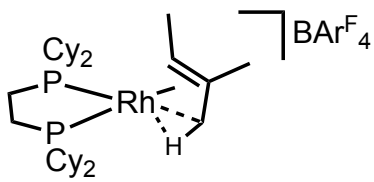

2-methylbut-2-ene

[1-methylbutenes][BArF $\left.{ }_{4}\right]$

\section{S.2.3.1. Synthesis of a methylbutenes complex, [1-methylbutenes][BAr ${ }_{4}$ ]}

For Solid-State NMR Spectroscopy and Single-Crystal X-Ray Diffraction: A J. Young flask $(\sim 50 \mathrm{~mL})$ was charged with ruby red crystals of [1-isoprene][BAr ${ }_{4}$ ] (100 mgs) and placed under an atmosphere of $\mathrm{H}_{2}$ (1 bar, $298 \mathrm{~K}, 25$ minutes). The colour of this material slowly turned dark red within this time. The powder was then exposed to a dynamic Schlenk line vacuum (< $2 \times 10^{-2}$ mbar, optimised at 4 hours $)$ to yield $\left[\mathrm{Rh}\left(\mathrm{Cy}_{2} \mathrm{PCH}_{2} \mathrm{CH}_{2} \mathrm{PCy}\right)\left(\mathrm{C}_{5} \mathrm{H}_{10}\right)\right]\left[\mathrm{BAr}_{4}\right]$, [1-methylbutenes][BAr $\left.{ }_{4}\right]$. The now yellow-orange powder was then taken into an argon filled glove box and stored at $-25^{\circ} \mathrm{C}$.

Note: This dehydrogenation reaction was also found to occur under a flushing argon flow and was complete within 6 hours (unoptimised).

A single-crystal X-ray diffraction experiment was conducted on a sample of these crystals and for further details of the structural refinement please refer to Section S.3. A sample of this material (45 mgs) was also packed in a $4.0 \mathrm{~mm}$ SSNMR rotor and analysed by ${ }^{31} \mathrm{P}\left\{{ }^{1} \mathrm{H}\right\}$ and ${ }^{13} \mathrm{C}\left\{{ }^{1} \mathrm{H}\right\}$ solid-state NMR spectroscopy at 294 and $158 \mathrm{~K}$. 


\section{S.2.3.2. Charaterisation data and spectra for [1-methylbutenes][BAr ${ }_{4}$ ]}

${ }^{1} \mathrm{H}$ NMR ( $\mathrm{CD}_{2} \mathrm{Cl}_{2}, 298 \mathrm{~K}, 400 \mathrm{MHz}$ ): $\delta 7.76$ (s, 8H, ortho-ArH), 7.61 (s, 4H, para-ArH), 2.051.68 (br m, overlapping aliphatic $\mathrm{CH}$ ), 1.61-0.85 (br m, aliphatic $\mathrm{CH}$ ).

${ }^{1} \mathrm{H}$ NMR ( $\mathrm{CD}_{2} \mathrm{Cl}_{2}, 183 \mathrm{~K}, 400 \mathrm{MHz}$ ): $\delta 7.77$ (s, 8H, ortho-ArH), 7.58 (s, 4H, para-ArH), 5.08 (s, $2 \mathrm{H}, \mathrm{C}=\mathrm{CHCH}_{3}$ from 2-methylbut-2-ene isomer), 4.33 (s, 1H, C= $\mathrm{CH}_{2}$ from 2-methylbut-1ene isomer), $3.29\left(\mathrm{~s}, 1 \mathrm{H}, \mathrm{C}=\mathrm{CH}_{2}\right.$ from 2-methylbut-1-ene isomer), 2.14-0.78 (br m, overlapping aliphatic $\mathrm{CH}$ ), -0.25 , (s, 3H, agostic from 2-methylbut-2-ene isomer), -1.23 (s, $1 \mathrm{H}$, agostic from 2-methylbut-1-ene isomer).

Note: The low temperature limit was not reached at $183 \mathrm{~K}$ in the ${ }^{1} \mathrm{H}$ NMR spectrum, so integrated values are approximate. The assignments for the above data have been made from ${ }^{1} \mathrm{H} /{ }^{1} \mathrm{H}$ COSY analysis upon the same sample, as well as comparison to the similar, previously reported isobutene ${ }^{\mathrm{s7}}$ and 2-butene ${ }^{\mathrm{s5}}$ coordinated complexes. NMR samples were prepared under argon and immediately run after preparation.

The ratio of coordinated 2-methylbut-1-ene: 2-methylbut-1-ene isomer is 1: 1.34 (Figure S16).

${ }^{31} \mathrm{P}\left\{{ }^{1} \mathrm{H}\right\}$ NMR $\left(\mathrm{CD}_{2} \mathrm{Cl}_{2}, 298 \mathrm{~K}, 162 \mathrm{MHz}\right): \delta 94.4\left(\mathrm{~d}, \mathrm{~J}_{\mathrm{RhP}} 181.7 \mathrm{~Hz}\right)$.

${ }^{31} \mathrm{P}\left\{{ }^{1} \mathrm{H}\right\}$ NMR $\left(\mathrm{CD}_{2} \mathrm{Cl}_{2}, 183 \mathrm{~K}, 162 \mathrm{MHz}\right)$ : 2-methylbut-2-ene Isomer: $\delta 96.6\left(\mathrm{~d}\right.$ of $\mathrm{d}, \mathrm{J}_{\mathrm{RhP}}=$ 199.6 Hz, $J_{\mathrm{PP}}=25 \mathrm{~Hz}$ ), $93.9\left(\mathrm{~d}\right.$ of $\mathrm{d}, J_{\mathrm{RhP}}=155.9 \mathrm{~Hz}, J_{\mathrm{PP}}=25 \mathrm{~Hz}$ ). 2-methylbut-1-ene Isomer: $\delta 95.2\left(\mathrm{~d}\right.$ of $\mathrm{d}, J_{\mathrm{RhP}}=152.6 \mathrm{~Hz}, J_{\mathrm{PP}}=26 \mathrm{~Hz}$ ), $93.9\left(\mathrm{~d}\right.$ of $\mathrm{d}, J_{\mathrm{RhP}}=212.0 \mathrm{~Hz}, J_{\mathrm{PP}}=26$ $\mathrm{Hz})$.

${ }^{19} \mathrm{~F}\left\{{ }^{1} \mathrm{H}\right\}$ NMR $\left(\mathrm{CD}_{2} \mathrm{Cl}_{2}, 298 \mathrm{~K}, 377 \mathrm{MHz}\right): \delta-62.9(\mathrm{~s})$.

${ }^{13} \mathrm{C}\left\{{ }^{1} \mathrm{H}\right\}$ NMR $\left(\mathrm{CD}_{2} \mathrm{Cl}_{2}, 183 \mathrm{~K}, 126 \mathrm{MHz}\right): \delta 161.6$ (q, J $\mathrm{JB}_{\mathrm{B}}=50 \mathrm{~Hz}$, ipso-ArC), 134.6 (s, ortho$\operatorname{ArC}$ ), 128.6 (q, $J_{C F}=32 \mathrm{~Hz}$, meta-ArC), 124.5 (q, $J_{C F}=272 \mathrm{~Hz},-\mathrm{CF}_{3}$ ), 119.1 (s, alkene-CH), 117.6 (s, para-ArC), 104.8 (s, alkene-CH), 86.4 (s, alkene-CH), 72 (br., alkene-CH), 37.4 to 19.8 (multiple aliphatic resonances from both isomers), 17.4, 17.2, 14.5, 13.7, 12.4, 11.7.

${ }^{31} \mathrm{P}\left\{{ }^{1} \mathrm{H}\right\}$ SSNMR (162 MHz, $294 \mathrm{~K}, 10 \mathrm{kHz}$ spin rate): $\delta 95.4$ (br, apparent triplet). ${ }^{31} \mathrm{P}\left\{{ }^{1} \mathrm{H}\right\}$ SSNMR (162 MHz, $158 \mathrm{~K}, 10 \mathrm{kHz}$ spin rate): $\delta 94.6$ (br, apparent triplet). 
${ }^{13} \mathrm{C}\left\{{ }^{1} \mathrm{H}\right\}$ SSNMR (101 MHz, $294 \mathrm{~K}, 10 \mathrm{kHz}$ spin rate): $\delta 163.8$ (ipso-ArC), 134.5 (ortho-ArC), 130.1 (meta-ArC), 124.6 (br, $\mathrm{CF}_{3}$ ), 118.2 (para-ArC), 115.5 (para-ArC), 38.4 (CH), 35.9 $(\mathrm{CH}), 30.6\left(\mathrm{CH}_{2}\right), 21.7\left(\mathrm{CH}_{2}\right), 13.1\left(\mathrm{CH}_{2}\right)$.

${ }^{13} \mathrm{C}\left\{{ }^{1} \mathrm{H}\right\}$ SSNMR (101 MHz, $158 \mathrm{~K}, 10 \mathrm{kHz}$ spin rate): $\delta 163.9$ (ipso-ArC), 133.9 (ortho-ArC), 129.4 (meta-ArC), 124.2 (br, CF3), 117.7 (para-ArC), 114.9 (para-ArC), 106.5 (C=C), 87.6 $(\mathrm{C}=\mathrm{C}), 69.5(\mathrm{C}=\mathrm{C}, 1+1$ coincidence $), 38.8(\mathrm{CH}), 36.6(\mathrm{CH}), 30.8\left(\mathrm{CH}_{2}\right), 26.6\left(\mathrm{CH}_{2}\right), 24.7$ $\left(\mathrm{CH}_{2}\right), 19.2\left(\mathrm{CH}_{2}\right), 16.8\left(\mathrm{CH}_{2}\right), 12.6\left(\mathrm{CH}_{2}\right)$.

ESI-MS: Not stable under the conditions during mass spectrometry (20 eV, $333 \mathrm{~K})$. Species with appropriate isotopic distributions at $\mathrm{m} / \mathrm{z}$ found $=581.240$, calculated to $\left[\left(\mathrm{Cy}_{2} \mathrm{PCH}_{2} \mathrm{CH}_{2} \mathrm{PCy}\right) \mathrm{Rh}\left(\mathrm{N}_{2}\right)_{2}\right]^{+}$(581.242). There is no evidence for the nitrogen compound in bulk samples so it is assumed to form via an in situ ESI-MS process.

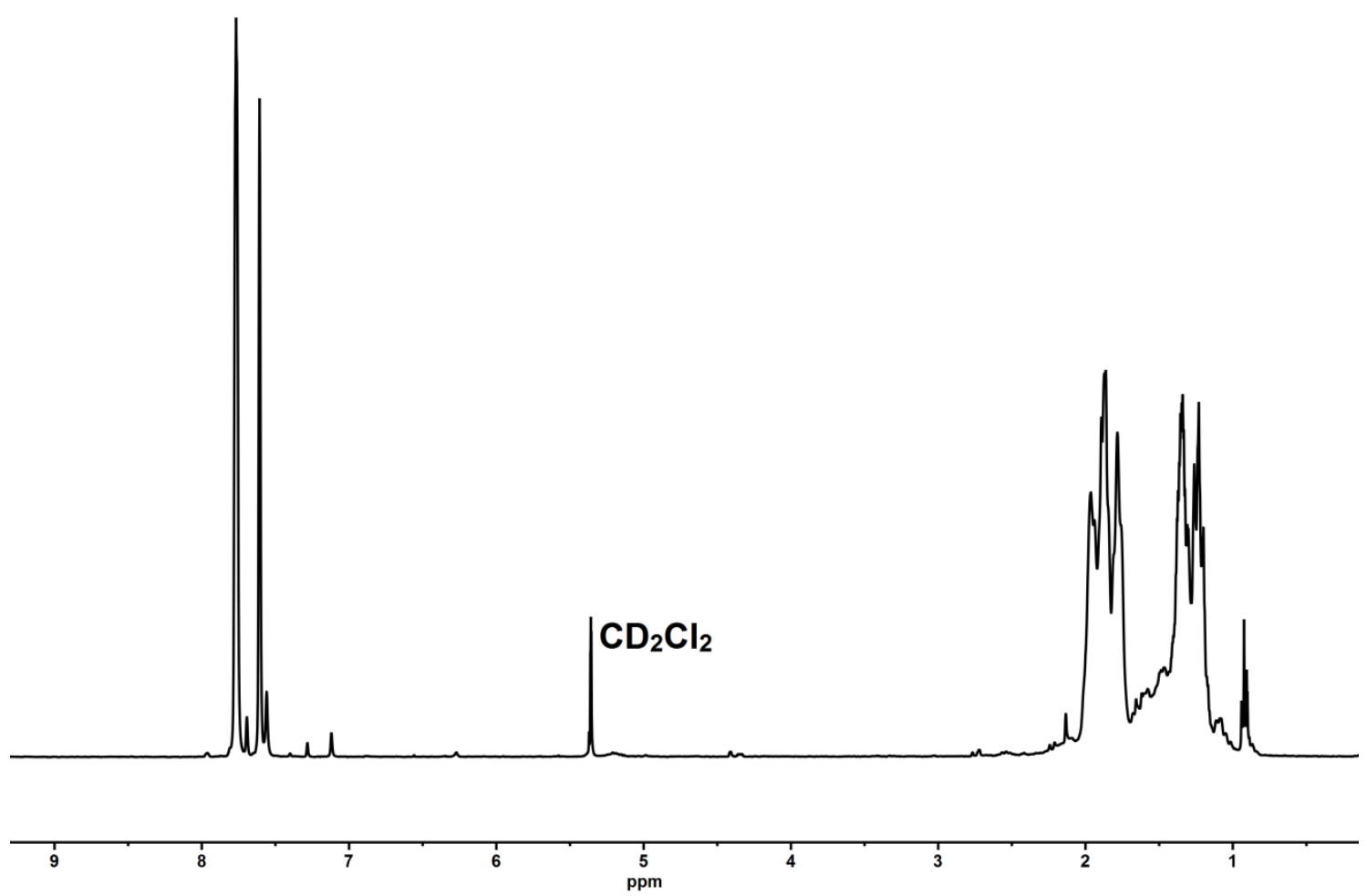

Figure S12: The solution ${ }^{1} \mathrm{H}$ NMR spectrum $\left(\mathrm{CD}_{2} \mathrm{Cl}_{2}, 298 \mathrm{~K}, 400 \mathrm{MHz}\right)$ of [1-methylbutenes][BAr$\left.{ }_{4}{ }\right]$. 
${ }^{1} \mathrm{H}$ NMR, $183 \mathrm{~K}$

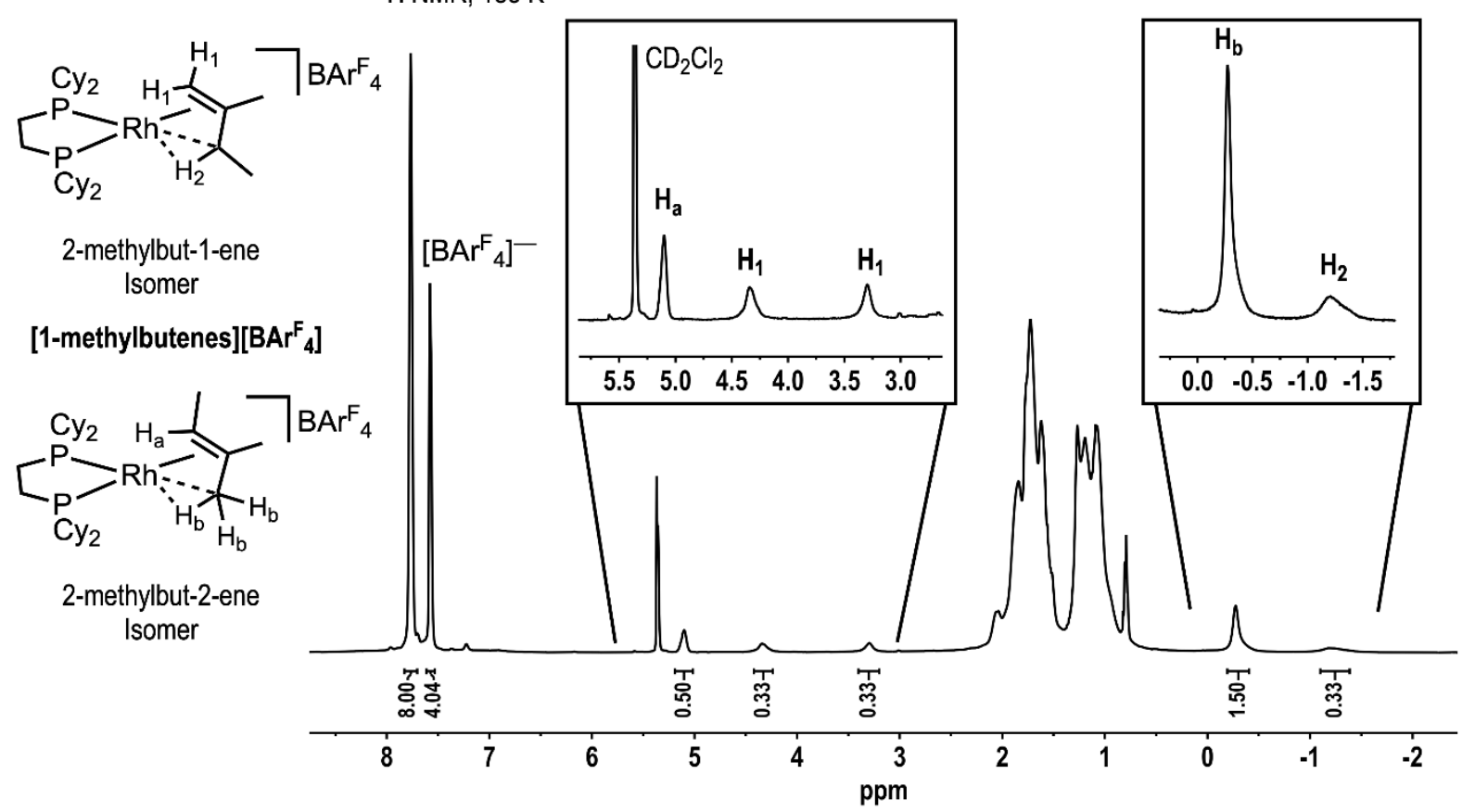

Figure S13: The solution ${ }^{1} \mathrm{H}$ NMR spectrum $\left(\mathrm{CD}_{2} \mathrm{Cl}_{2}, 183 \mathrm{~K}, 400 \mathrm{MHz}\right)$ of [1-methylbutenes][BAr${ }_{4}$ ]. The inserts show enlargements of the peaks between $\delta 5.5$ and 3.0 and 0.00 to -1.23 . The ratio of 2-methylbut-1-ene: 2-methylbut-2-ene isomers is 1: 1.34, from the integration of the signals of $\mathrm{H}_{1}: \mathrm{H}_{\mathrm{a}}$. 


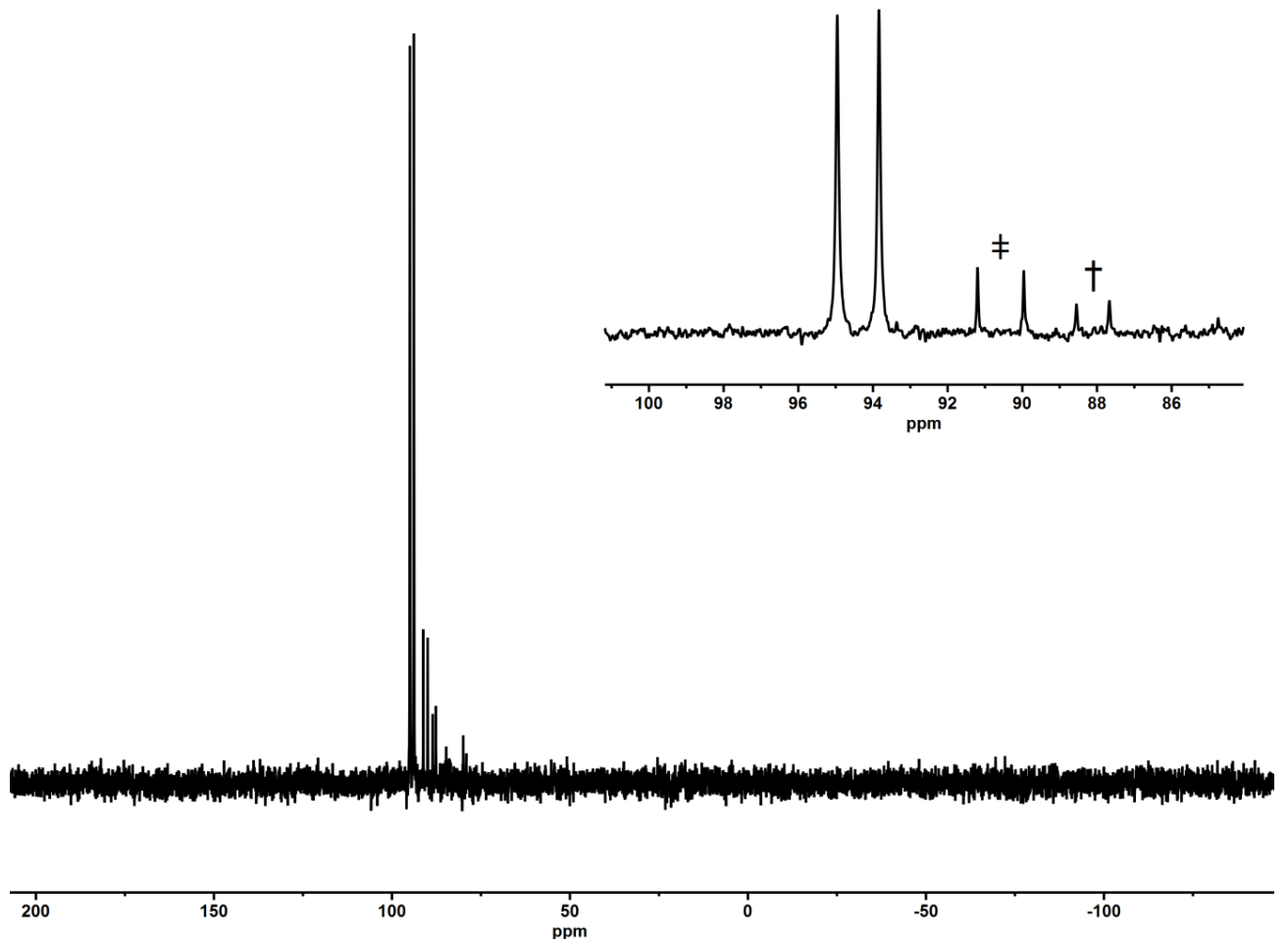

Figure S14: The solution ${ }^{31} \mathrm{P}\left\{{ }^{1} \mathrm{H}\right\} \quad \mathrm{NM} R \mathrm{ppm}$ spectrum $\left(\mathrm{CD}_{2} \mathrm{Cl}_{2}, 298 \mathrm{~K}, 162 \mathrm{MHz}\right)$ of [1-methylbutenes][BAr${ }_{4}$ ]. The inset is an enlargement of the resonances between $\delta 100$ and 86. $\ddagger$ is of [1-BArF ${ }_{4}$, ${ }^{\mathrm{S} 2}$ formed as a minor product during the initial hydrogenation reaction. The signal marked $\dagger$ is from a further decomposition product of [1-BAr ${ }_{4}$ ] in solution. ${ }^{\mathrm{S} 2}$

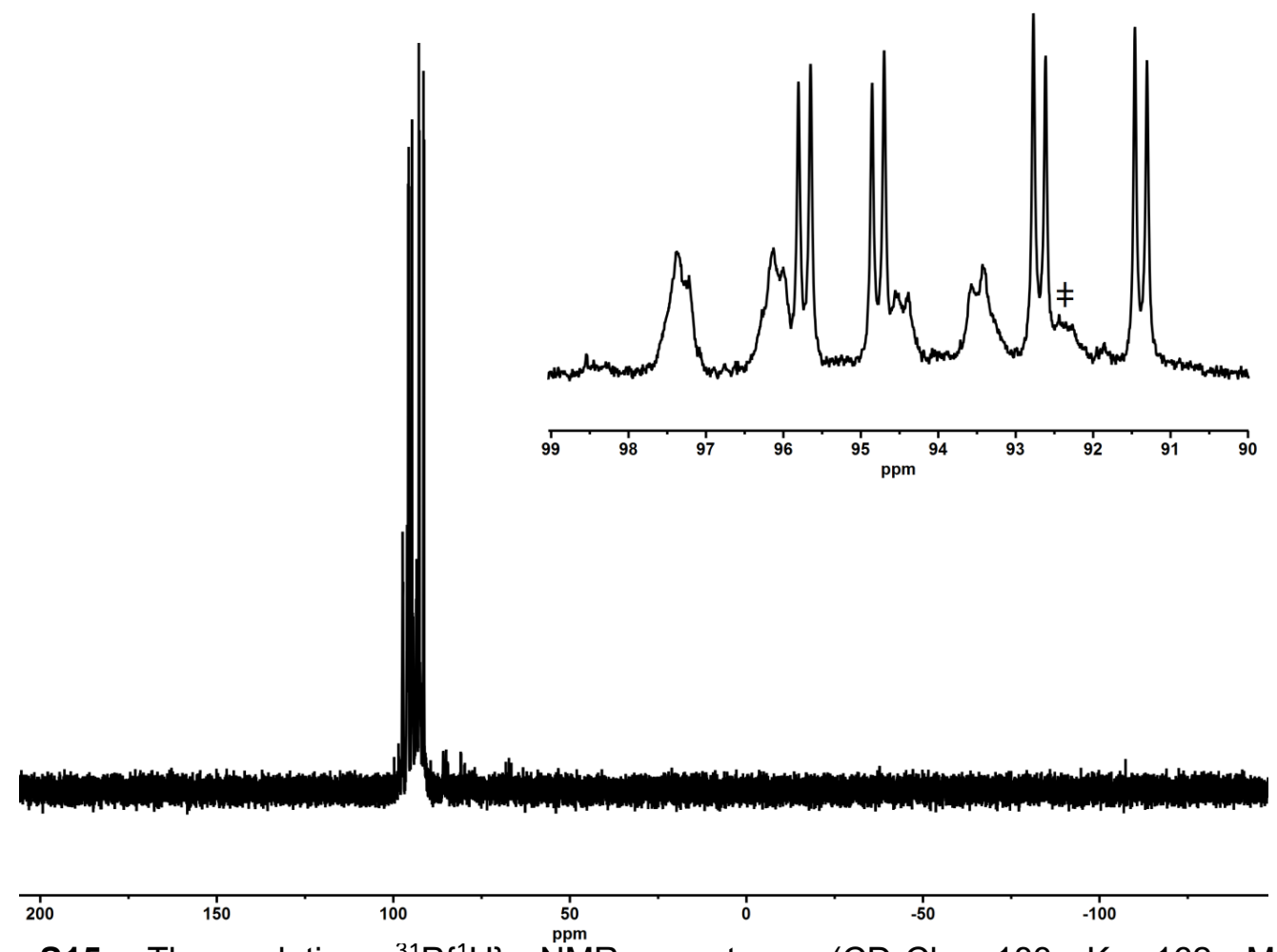

Figure S15: The solution ${ }^{31} \mathrm{P}\left\{{ }^{1} \mathrm{H}\right\} \quad$ NMR spectrum $\left(\mathrm{CD}_{2} \mathrm{Cl}_{2}, 183 \mathrm{~K}, 162 \mathrm{MHz}\right)$ of [1-methylbutenes][BAr ${ }_{4}$ ]. The inset is an enlargement of the resonances between $\delta 99$ and 90 . The signal marked $\ddagger$ is from a further decomposition product of [1-BAr${ }_{4}$ ] in solution. ${ }^{\mathrm{S} 2}$ 

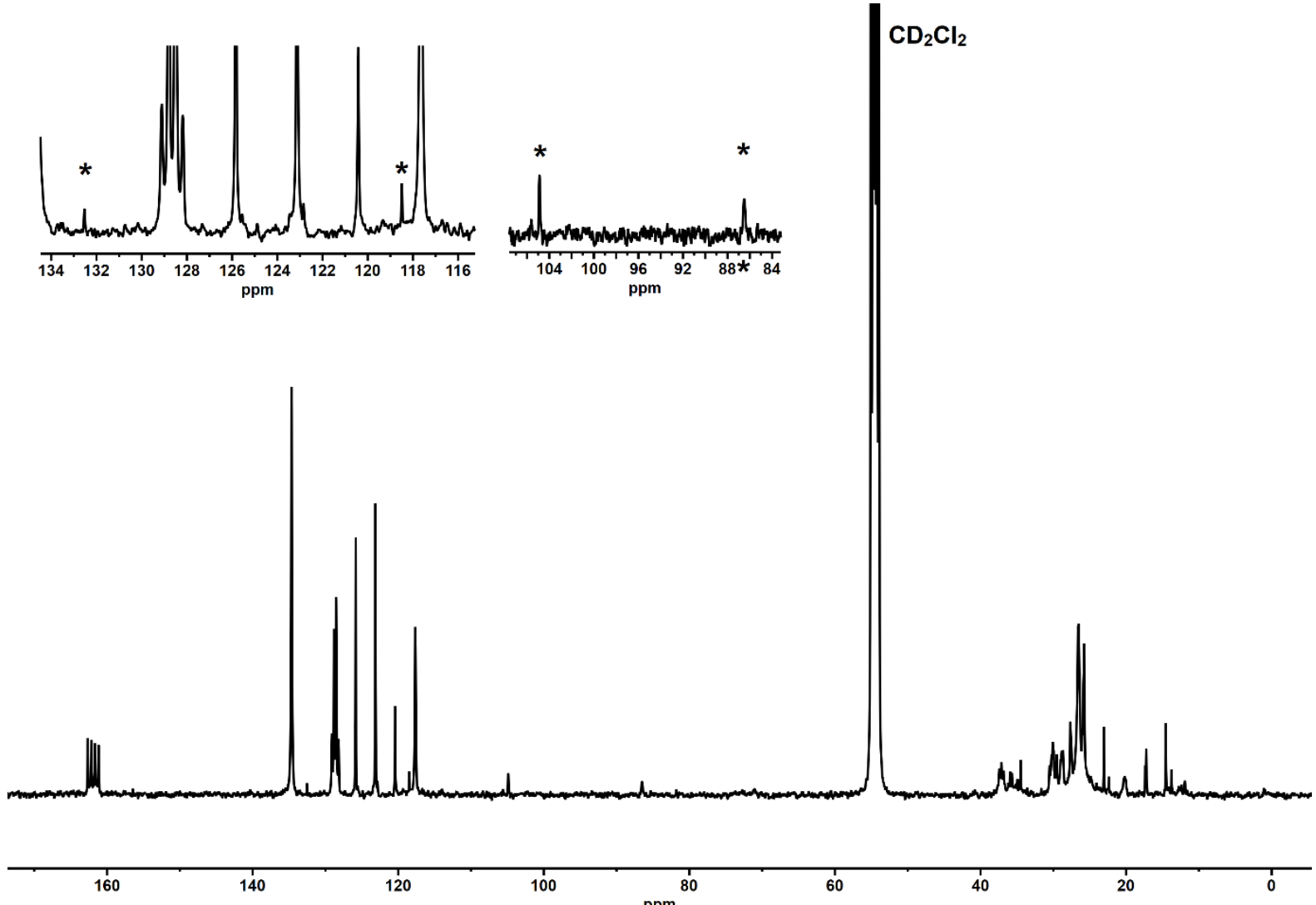

Figure S16: The solution ${ }^{13} \mathrm{C}\left\{{ }^{1} \mathrm{H}\right\}$ NMR spectrum $\left(\mathrm{CD}_{2} \mathrm{Cl}_{2}, 183 \mathrm{~K}, 126 \mathrm{MHz}\right)$ of [1-methylbutenes][BAr${ }_{4}{ }_{4}$. The inset is an enlargement of the alkene resonances between $\delta 134$ to 116 and 104 to 84 . Signals assigned to alkene carbons labelled *. 


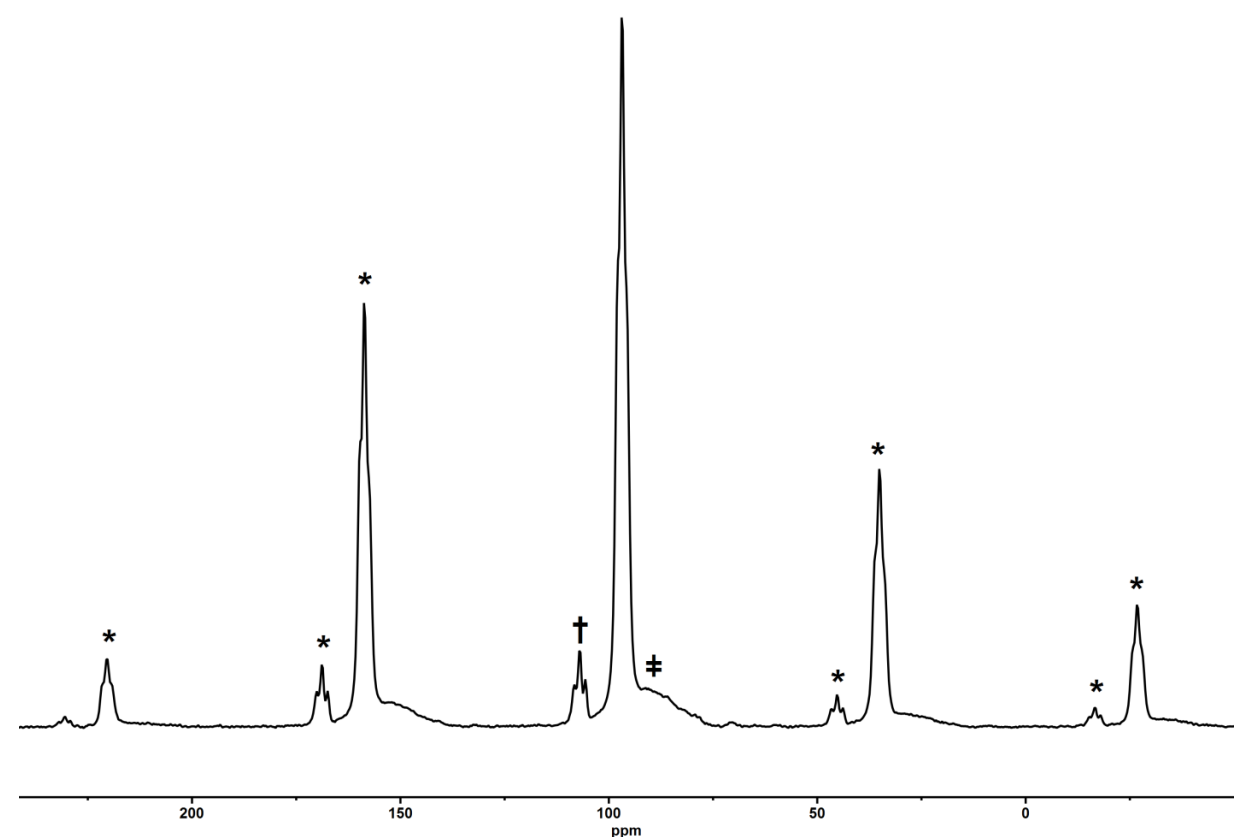

Figure S17: The ${ }^{31} \mathrm{P}\left\{{ }^{1} \mathrm{H}\right\}$ SSNMR spectrum (162 MHz, $294 \mathrm{~K}, 10 \mathrm{kHz}$ spin rate) of [1-methylbutenes][BArF ${ }_{4}$ ] isomers. Peaks marked * are spinning sidebands, $\neq$ are assigned to [1-BAr $\left.{ }_{4}\right]^{\mathrm{S} 2}$ and $\dagger$ to an unidentified, previously noted, decomposition product under an hydrogen atmosphere. $^{\text {S7 }}$

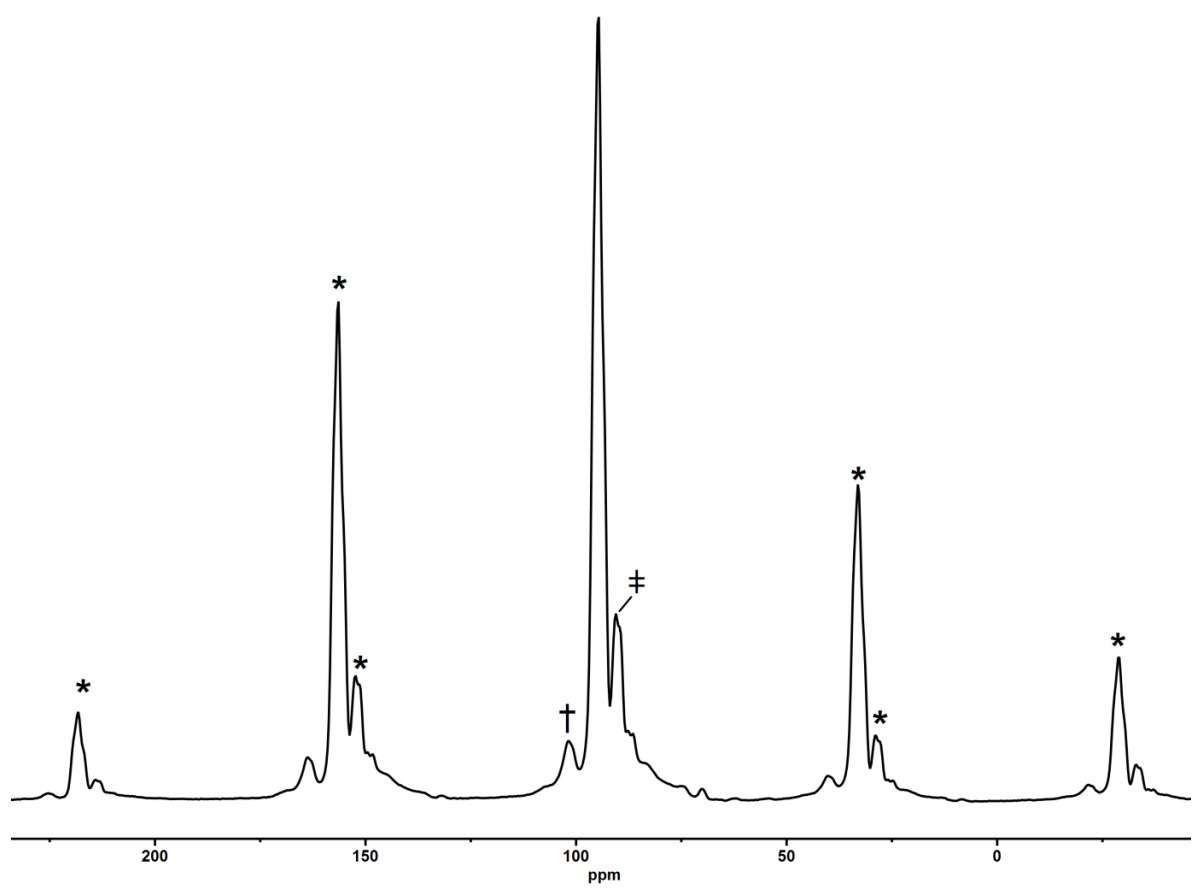

Figure S18: The ${ }^{31} \mathrm{P}\left\{{ }^{1} \mathrm{H}\right\}$ SSNMR spectrum (162 MHz, $158 \mathrm{~K}, 10 \mathrm{kHz}$ spin rate) of [1-methylbutenes][BAr ${ }_{4}$ ] isomers. Peaks marked ${ }^{*}$ are spinning sidebands, $\neq$ is assigned to [1-BArF ${ }_{4}$ ], ${ }^{\mathrm{S} 2}$ but we cannot rule out this from a static [1-methylbutenes][BArF ${ }_{4}$ ] isomer. $\dagger$ is assigned to an unidentified, previously noted, decomposition product under a hydrogen atmosphere. ${ }^{S 7}$ Note: The spectrum in Figures S20 to S21 were obtained from a different sample to that of Figures $\mathrm{S} 13$ and S14 resulting in a different degree of decomposition to [1-BArF ${ }_{4}$. 


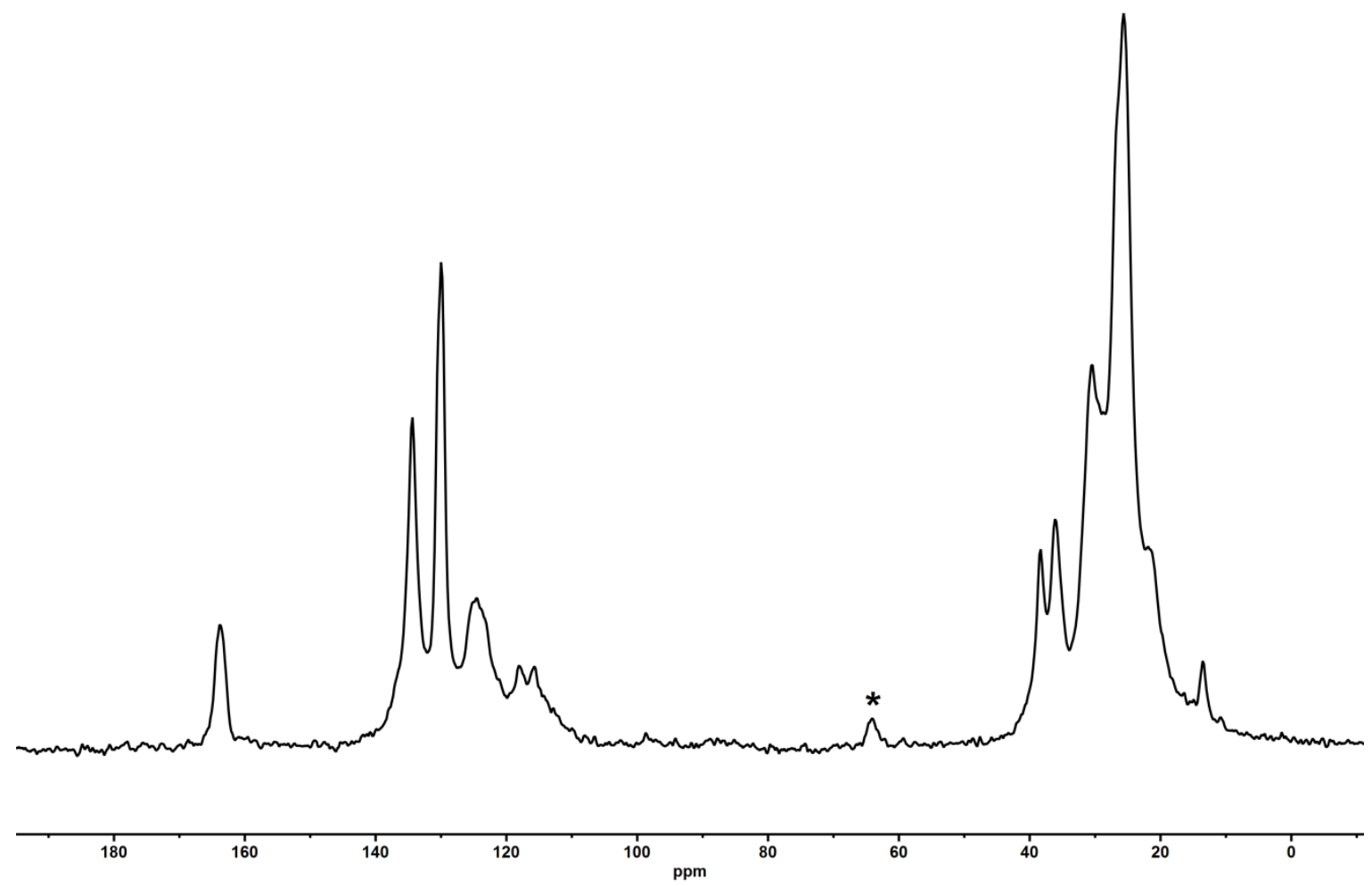

Figure S19: The ${ }^{13} \mathrm{C}\left\{{ }^{1} \mathrm{H}\right\}$ SSNMR spectrum (101 MHz, $294 \mathrm{~K}, 10 \mathrm{kHz}$ spin rate) of [1-methylbutenes][BAr$\left.{ }_{4}\right]$. The resonances marked * are due to spinning sidebands.

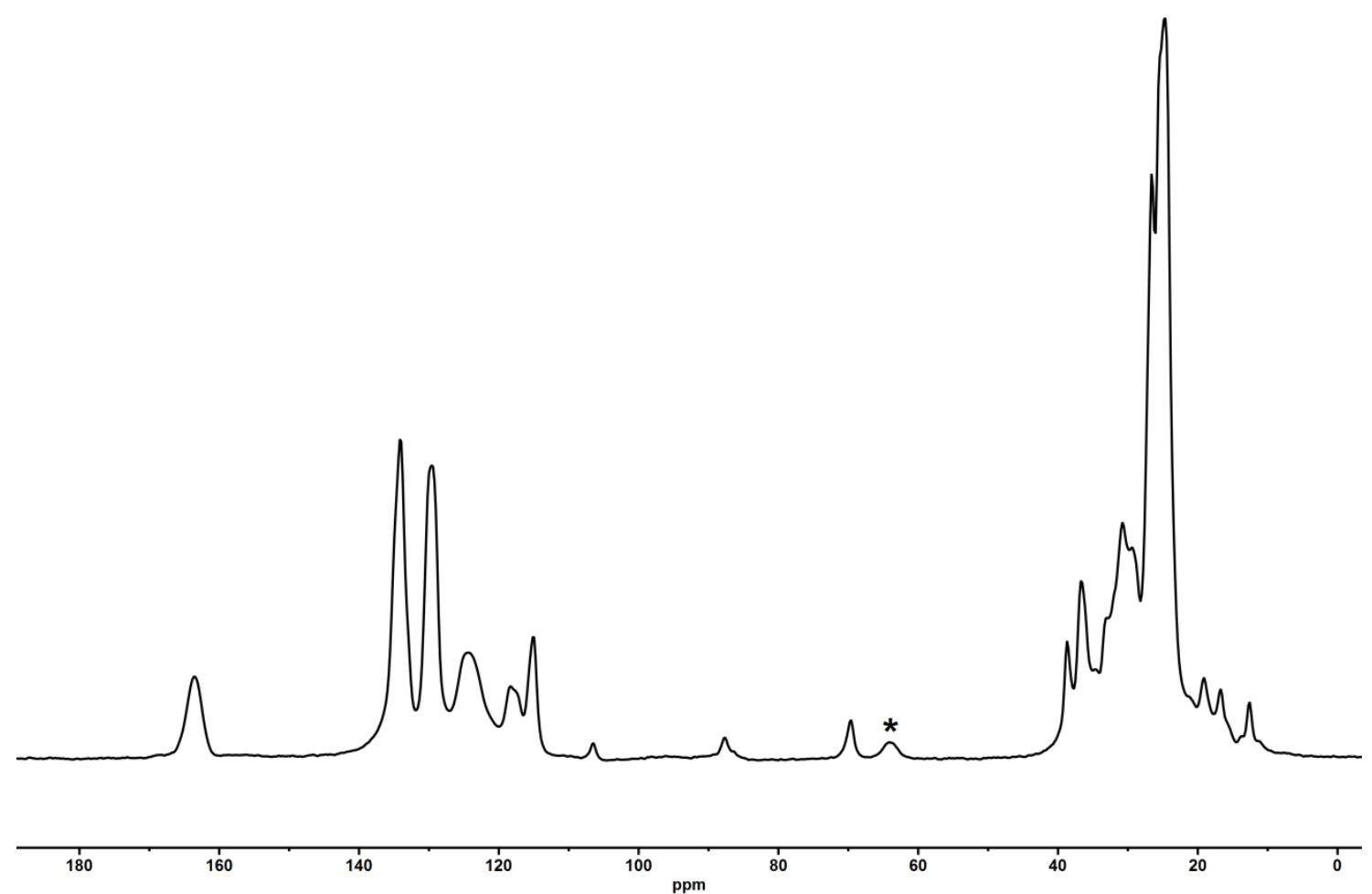

Figure S20: The ${ }^{13} \mathrm{C}\left\{{ }^{1} \mathrm{H}\right\}$ SSNMR spectrum (101 MHz, $158 \mathrm{~K}, 10 \mathrm{kHz}$ spin rate) of [1-methylbutenes][BAr${ }_{4}$ ]. The resonances marked * are due to spinning sidebands. 


\section{S.2.3.3. Liberation of bound methylbutenes from [1-methylbutenes][BArF $\left.{ }_{4}\right]$}

A J. Young flask was charged with a sample of [1-methylbutenes][BAr $\left.{ }_{4}\right]$ (30 mgs), as prepared from the method in Section S.2.4.1. The flask was placed under vacuum $(<2 \times$ $10^{-2} \mathrm{mbar}$ ) and backfilled with CO (1 bar, $298 \mathrm{~K}$ ). After 18 hours, the orange crystalline material had turned a pale-yellow colour. The resultant solid was confirmed to be $\left[\mathrm{Rh}\left(\mathrm{Cy}_{2} \mathrm{PCH}_{2} \mathrm{CH}_{2} \mathrm{PCy}\right)(\mathrm{CO})_{2}\right]\left[\mathrm{BAr}_{4}{ }_{4}\right]$ by solution ${ }^{31} \mathrm{P}\left\{{ }^{1} \mathrm{H}\right\}$ NMR spectroscopy $\left(\delta 85.22, \mathrm{~d}, \mathrm{~J}_{\mathrm{RhP}}\right.$ $=116 \mathrm{~Hz}) .{ }^{\mathrm{S} 5}$ The volatile component was then isolated by trap-to-trap distillation into $\mathrm{CD}_{2} \mathrm{Cl}_{2}$ and analysed by solution ${ }^{1} \mathrm{H}$ NMR spectroscopy.

2-methylbut-1-ene: ${ }^{1} \mathrm{H}$ NMR $\left(\mathrm{CDCl}_{3}, 298 \mathrm{~K}, 400 \mathrm{MHz}\right.$ ): $\delta 4.66$ (s., $2 \mathrm{H}$ ), 2.02 (quartet, $2 \mathrm{H}, \mathrm{J}_{\mathrm{HH}}$ $=7.5 \mathrm{~Hz}$ ), 1.73 (s., 3H), 1.03 (triplet, $3 \mathrm{H}, J_{\mathrm{HH}}=7.5 \mathrm{~Hz}$ ).

2-methylbut-2-ene: ${ }^{1} \mathrm{H}$ NMR $\left(\mathrm{CH}_{2} \mathrm{Cl}_{2}, 298 \mathrm{~K}, 500 \mathrm{MHz}\right): \delta 5.18(\mathrm{~m} ., 1 \mathrm{H}), 1.67$ (s., 3H), 1.60 (s., $3 \mathrm{H}$ ), 1.55 (d., $3 \mathrm{H}, \mathrm{J}_{\mathrm{HH}}=6.7 \mathrm{~Hz}$ ).

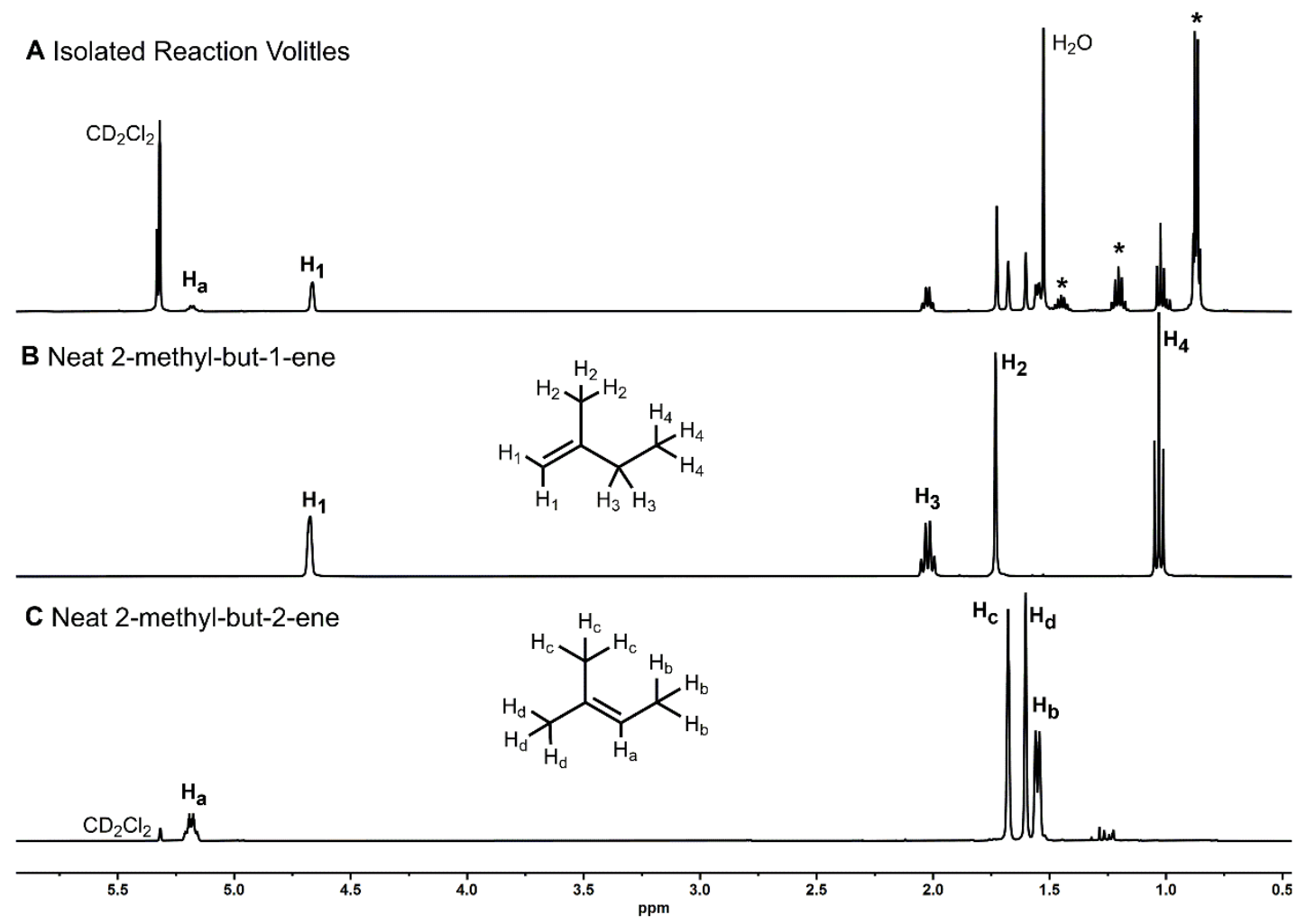

Figure S21: The solution ${ }^{1} \mathrm{H}$ NMR spectra of $\mathbf{A}:\left(\mathrm{CD}_{2} \mathrm{Cl}_{2}, 298 \mathrm{~K}, 500 \mathrm{MHz}\right)$ liberated reaction volatiles showing mix of methylbutene isomers. Peaks marked * are from 2-methylbutane from alkane liberation from sample decomposition during the initial hydrogenation. $\mathbf{B}$ : $\left(\mathrm{CDCl}_{3}, 298 \mathrm{~K}, 400 \mathrm{MHz}\right)$ Neat 2-methyl-but-1-ene. C: $\left(\mathrm{CD}_{2} \mathrm{Cl}_{2}, 298 \mathrm{~K}, 400 \mathrm{MHz}\right)$ Neat 2methylbut-2-ene. Note: The integration of the $\mathrm{H}_{1}$ : $\mathrm{H}_{a}$ resonances (from spectrum $A$ ) is 2.79:

1. This suggests a 1.4: 1 ratio of liberated 2-methylbut-1-ene: 2-methylbut-2-ene. 


\section{S.2.3.4. Reaction kinetics of the dehydrogenation of [1-(2-methylbutane)][BAr $\left.{ }_{4}\right]$}

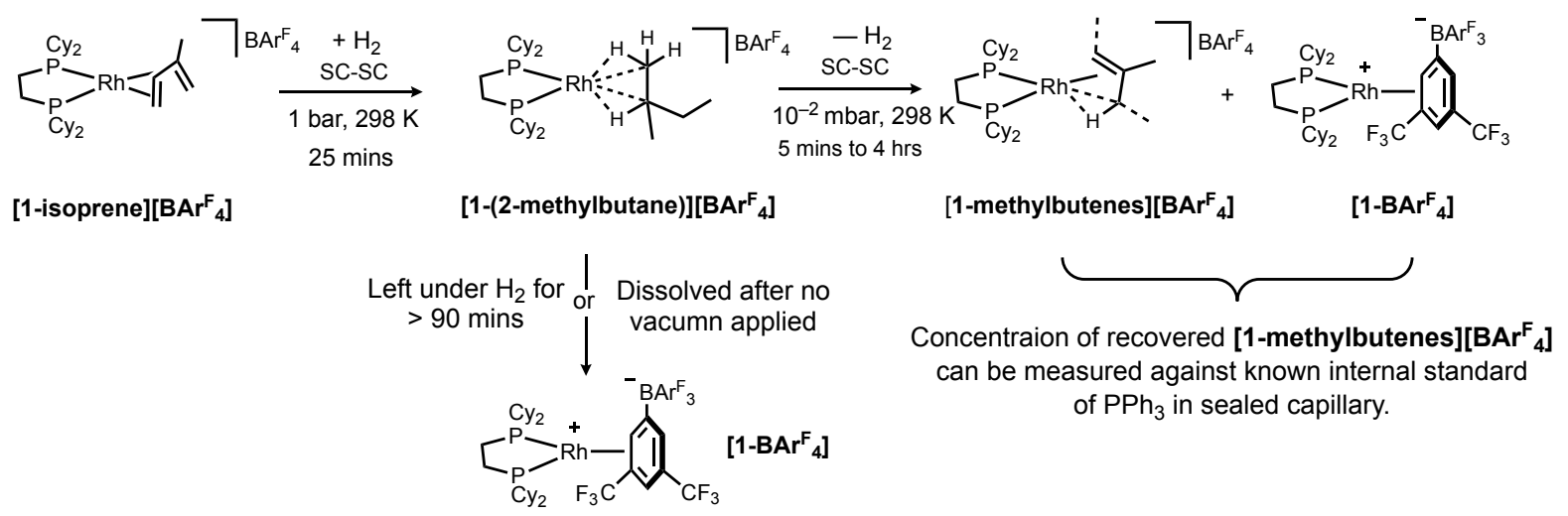

Procedure: Nine J. Young NMR tubes $(\sim 2.1 \mathrm{~mL})$ were individually charged with 12.5 mgs $(8.59 \mu \mathrm{mol})$ of powdered crystalline [1-isoprene][BAr${ }_{4}$ ] and a separate sealed capillary containing $\mathrm{PPh}_{3}$ in acetone. The tubes were evacuated $\left(<2 \times 10^{-2} \mathrm{mbar}\right)$ and $\mathrm{H}_{2}$ ( 1 bar, 298 K) was added for 25 minutes, to yield the dark red powder of [1-(2-methylbutane)][BAr $\left.{ }_{4}\right]$. These samples were placed under vacuum $\left(<2 \times 10^{-2} \mathrm{mbar}\right)$ for varying times between 0 seconds and 4 hours at room temperature, then dissolved in $\mathrm{CH}_{2} \mathrm{Cl}_{2}(0.35 \mathrm{~mL})$ and analysed by solution ${ }^{31} \mathrm{P}\left\{{ }^{1} \mathrm{H}\right\}$ NMR spectroscopy.

When time $=0$ seconds, no signals relating to [1-isoprene][BAr${ }_{4}$ ] could be identified, confirming the complete hydrogenation of the sample. When left under vacuum for greater than 4 hours (with longest time point of 7 days), no signals relating to [1-isoprene][BAr ${ }_{4}$ ] were identified confirming only the selective dehydrogenation to [1-methylbutenes][BAr ${ }_{4}$ ].

After dissolution in $\mathrm{CH}_{2} \mathrm{Cl}_{2}$, two major signals were observed in the ${ }^{31} \mathrm{P}\left\{{ }^{1} \mathrm{H}\right\} \mathrm{NMR}$ spectra at $298 \mathrm{~K}$, of the dehydrogenation product [1-methylbutenes][BAr$\left.{ }_{4}\right]\left(\delta 94, J_{R h P}=179 \mathrm{~Hz}\right)$ and decomposition product [1-BAr $\left.{ }_{4}\right]\left(\delta 91, J_{\mathrm{RhP}}=201 \mathrm{~Hz}\right)$. The concentration of recovered [1-methylbutenes][BAr${ }_{4}$ ] could be accurately measured against the integration of the $\mathrm{PPh}_{3}$ signal.

No more than $82 \%$ recovered [1-methylbutenes][BAr ${ }_{4}$ ] could be achieved relative to the initial [1-isoprene][BAr${ }_{4}$ ] loading. This suggests a consistent $\sim 20 \%$ decomposition to [1-BArF ${ }_{4}$ ] during the initial 25 minutes hydrogenation time. This $\sim 20 \%$ decomposition is also observed in the ${ }^{31} \mathrm{P}\left\{{ }^{1} \mathrm{H}\right\}$ SSNMR spectrum of [1-(2-methylbutane)][BAr ${ }_{4}$ ] (Figure S13). 


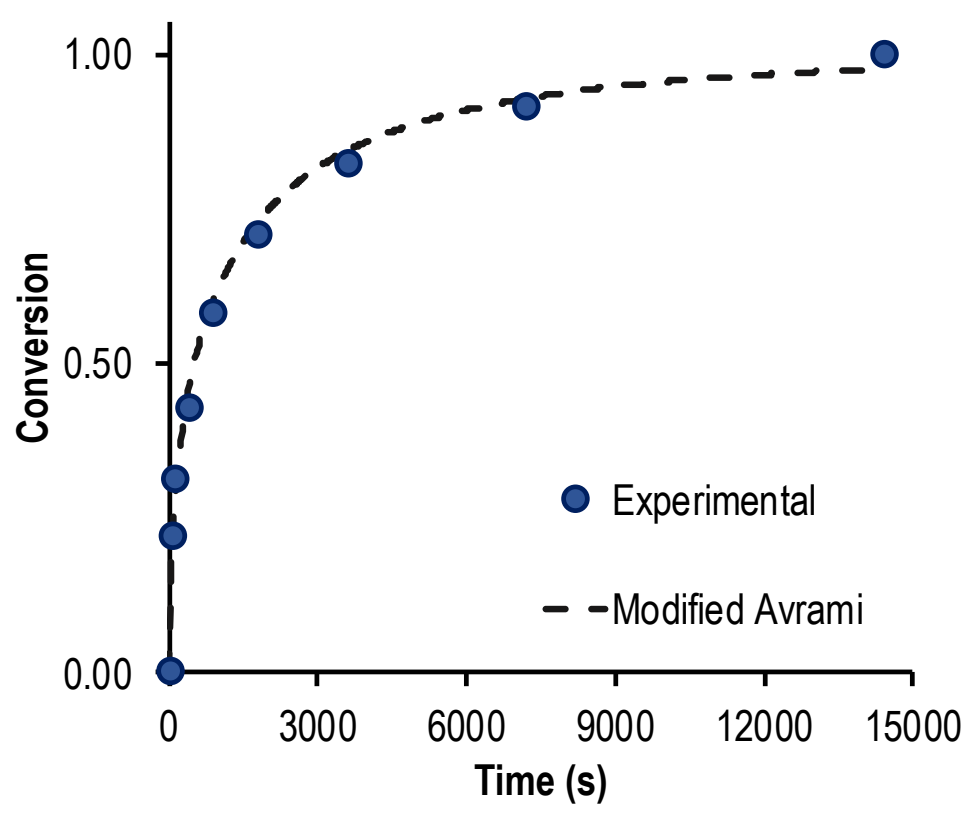

Figure S22: Temporal profile for the dehydrogenation of [1-(2-methylbutane)][BAr ${ }_{4}$ ]. Each data point represents an individual dehydrogenation experiments and are shown by blue markers. These plots have been modelled to take into account the $20 \%$ systematic decomposition of [1-(2-methylbutane)][BArF $\left.{ }_{4}\right]$ observed during the initial hydrogenation (See Section S.2.3.3). The maximum concentration formed of [1-methylbutenes][BAr ${ }_{4}$ ] is $20.16 \mathrm{mM}$.

Overlaid curve represents the modified JAMK plot of conversion ${ }^{\mathrm{S} 8}(f)=\left(1-\mathrm{e}^{(-k t)^{\wedge} \mathrm{n}}\right)$ versus time for the dehydrogenation of [1-(2-methylbutane)][BAr ${ }_{4}$ ] to [1-methylbutenes][BAr ${ }_{4}$ ] in the solid-state. Where $k=$ growth rate constant, $9.5 \times 10^{-4}\left( \pm 6 \times 10^{-5}\right) \mathrm{s}^{-1}$, and $\mathrm{n}=$ Avrami exponent $(0.50 \pm 0.02)$. 


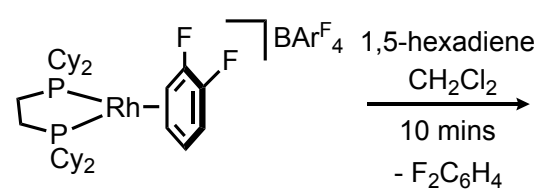

Solution
Only<smiles>CC=CCCC</smiles>

2,4-isomer

$\left[1-\mathrm{F}_{2} \mathrm{C}_{6} \mathrm{H}_{4}\right]\left[\mathrm{BAr}_{4}\right]$

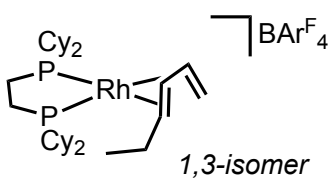

[1-hexadiene][BArF $\left.{ }_{4}\right]$

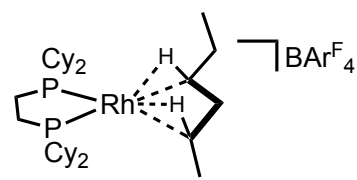

[1-hexane][BArF $\left.{ }_{4}\right]$ 2,4-isomer only

\section{S.2.4.1. Synthesis of a precursor hexane complex, [1-hexadiene][BAr ${ }_{4}$ ]}

A pale-yellow solution of $\left[1-\mathrm{F}_{2} \mathbf{C}_{6} \mathrm{H}_{4}\right]\left[\mathrm{BAr}_{4}{ }_{4}\right](600 \mathrm{mgs}, 0.40 \mathrm{mmol})$ in $\mathrm{CH}_{2} \mathrm{Cl}_{2}(20 \mathrm{~mL})$ was treated with neat 1,5 -hexadiene $(0.1 \mathrm{~mL}, 0.48 \mathrm{mmol})$ at ambient temperature. The resultant deep red solution was stirred at ambient temperature for 30 minutes. Pentane $(100 \mathrm{~mL})$ was then added with vigorous stirring which resulted in the formation of a red precipitate. The solid was isolated by filtration and washed with pentane $(2 \times 20 \mathrm{~mL})$. The solid was then dissolved in 1,2-dichloroethane ( $12 \mathrm{~mL})$ and filtered into a J. Young crystallisation flask and layered with pentane. Ruby red crystals were obtained after storage for 1 week at room temperature of $\left[\mathrm{Rh}\left(\mathrm{Cy}_{2} \mathrm{PCH}_{2} \mathrm{CH}_{2} \mathrm{PCy}_{2}\right)\left(\mathrm{C}_{6} \mathrm{H}_{10}\right)\right]\left[\mathrm{BAr}_{4}{ }_{4}\right]$, [1-hexadiene][BAr ${ }_{4}{ }_{4}$ ] (Yield: 404 mgs, $69 \%)$.

Note: The 2,4-isomer was found to be the only isomer present in the solid-state. Taking single-crystals of the 2,4-isomer and re-dissolving in $\mathrm{CH}_{2} \mathrm{Cl}_{2}$ initially showed only the 2,4-isomer, where the mixture of isomers was observed to grow in within 2 hours. 


\section{S.2.4.2. Charaterisation data and spectra for [1-hexadiene][BAr ${ }_{4}$ ]}

${ }^{1} \mathrm{H}$ NMR ( $\mathrm{CD}_{2} \mathrm{Cl}_{2}, 298 \mathrm{~K}, 400 \mathrm{MHz}$ ): $\delta 7.82$ (s, 8H, ortho-ArH), 7.67 (s, 4H, para-ArH), 5.68 (d. of d., alkene-H, $J=14.6,3.8 \mathrm{~Hz}$ ), 5.49 (d. of m., alkene-H, $J=12.5 \mathrm{~Hz}$ ), 5.34 (m., alkene-H, $J=1.1 \mathrm{~Hz}), 4.25(\mathrm{~m}$., alkene-H), 4.12 (m., alkene-H), 2.88 (d., alkene-H, $J=$ 14.4 Hz), 2.16 -1.70 (br m, overlapping aliphatic $\mathrm{CH}$ ), 1.52-0.98 (br m, aliphatic $\mathrm{CH}$ ).

${ }^{31} \mathrm{P}\left\{{ }^{1} \mathrm{H}\right\}$ NMR $\left(\mathrm{CD}_{2} \mathrm{Cl}_{2}, 298 \mathrm{~K}, 162 \mathrm{MHz}\right):$ 2,4-isomer: $\delta 80.6\left(\mathrm{~d}, J_{\mathrm{RhP}}=179 \mathrm{~Hz}\right)$. The 1,3-isomer: $\delta 84.5$ (d.d., $J_{\mathrm{RhP}}=174 \mathrm{~Hz}, J_{\mathrm{PP}}=22 \mathrm{~Hz}$ ), $\delta 77.3$ (d.d., $J_{\mathrm{RhP}}=171 \mathrm{~Hz}, J_{\mathrm{PP}}=22$ $\mathrm{Hz})$.

${ }^{19} \mathrm{~F}\left\{{ }^{1} \mathrm{H}\right\}$ NMR $\left(\mathrm{CD}_{2} \mathrm{Cl}_{2}, 298 \mathrm{~K}, 377 \mathrm{MHz}\right): \delta-62.9(\mathrm{~s})$.

${ }^{13} \mathrm{C}\left\{{ }^{1} \mathrm{H}\right\}$ NMR $\left(\mathrm{CD}_{2} \mathrm{Cl}_{2}, 298 \mathrm{~K}, 126 \mathrm{MHz}\right): \delta 162.1$ (q, $\mathrm{J}_{\mathrm{CB}}=50 \mathrm{~Hz}$, ipso-ArC), 135.1 (s, ortho$\operatorname{ArC}$ ), 129.2 (q, J JF $=32 \mathrm{~Hz}$, meta-ArC), 125.0 (q, J J $=272 \mathrm{~Hz},-\mathrm{CF}_{3}$ ), 117.7 (s, para-ArC), 108.2, 109.2, 104.6, 101.0, 98.6, 97.5, 88.8, 61.8 (m, multiple alkene resonances from 2,4-isomer and 1,3-isomers), 38.8, 38.5, 37.8, 37.0, 36.2, 35.5, 32.0 - 19.9 (m, overlapping cyclohexyl- $\mathrm{CH}_{2}$ from isomers $\mathrm{A}$ and $\mathrm{B}$ ).

ESI-MS (calc. for $\left.\left[\mathrm{Rh}\left(\mathrm{Cy}_{2} \mathrm{PCH}_{2} \mathrm{CH}_{2} \mathrm{PCy}_{2}\right)\left(\mathrm{C}_{6} \mathrm{H}_{10}\right)\right]^{+}\right): m / z 607.3086$ (607.3063).

Elemental Analysis (calc. for $\mathrm{C}_{64} \mathrm{H}_{70} \mathrm{BF}_{24} \mathrm{P}_{2} \mathrm{Rh}$ ): $\mathrm{C} 52.17$ (52.26), $\mathrm{H} 4.83$ (4.80).

${ }^{31} \mathrm{P}\left\{{ }^{1} \mathrm{H}\right\}$ SSNMR (162 MHz, $298 \mathrm{~K}, 10 \mathrm{kHz}$ spin rate): $\delta 82.5\left(\mathrm{~d}, J_{\mathrm{RhP}}=189 \mathrm{~Hz}\right), 74.5 .(\mathrm{d}$, $\left.J_{\mathrm{RhP}}=182 \mathrm{~Hz}\right)$.

${ }^{13} \mathrm{C}\left\{{ }^{1} \mathrm{H}\right\}$ SSNMR (101 MHz, $298 \mathrm{~K}, 10 \mathrm{kHz}$ spin rate): $\delta 164.7(\operatorname{ArC}), 161.3(\operatorname{ArC}), 134.8$ $(\operatorname{ArC}), 129.0(\operatorname{ArC}), 124.4(\operatorname{ArC}), 117.7(\operatorname{ArC}), 115.1(\operatorname{ArC}), 99.3(C=C), 98.6(C=C), 88.6$ $\mathrm{C}=\mathrm{C}), 85.3(\mathrm{C}=\mathrm{C}), 39.6,38.1,34.9,32.5,30.6,29.2,27.0,26.0,24.6,22.6,19.9,17.0$ (multiple aliphatic resonances). 


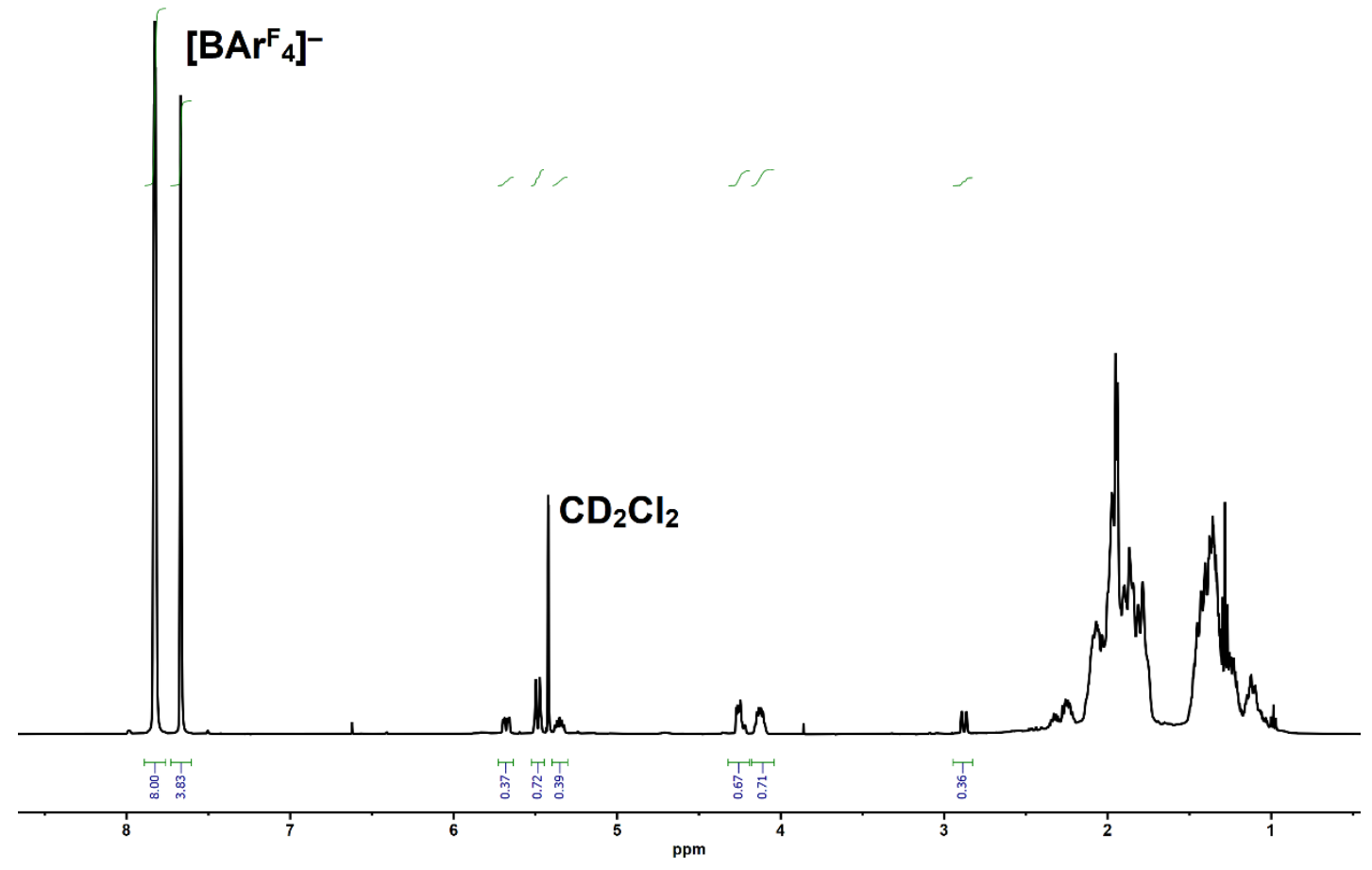

Figure S23: The solution ${ }^{1} \mathrm{H}$ NMR spectrum $\left(\mathrm{CD}_{2} \mathrm{Cl}_{2}, 298 \mathrm{~K}, 400 \mathrm{MHz}\right)$ of [1-hexadiene][BAr ${ }_{4}$ ].
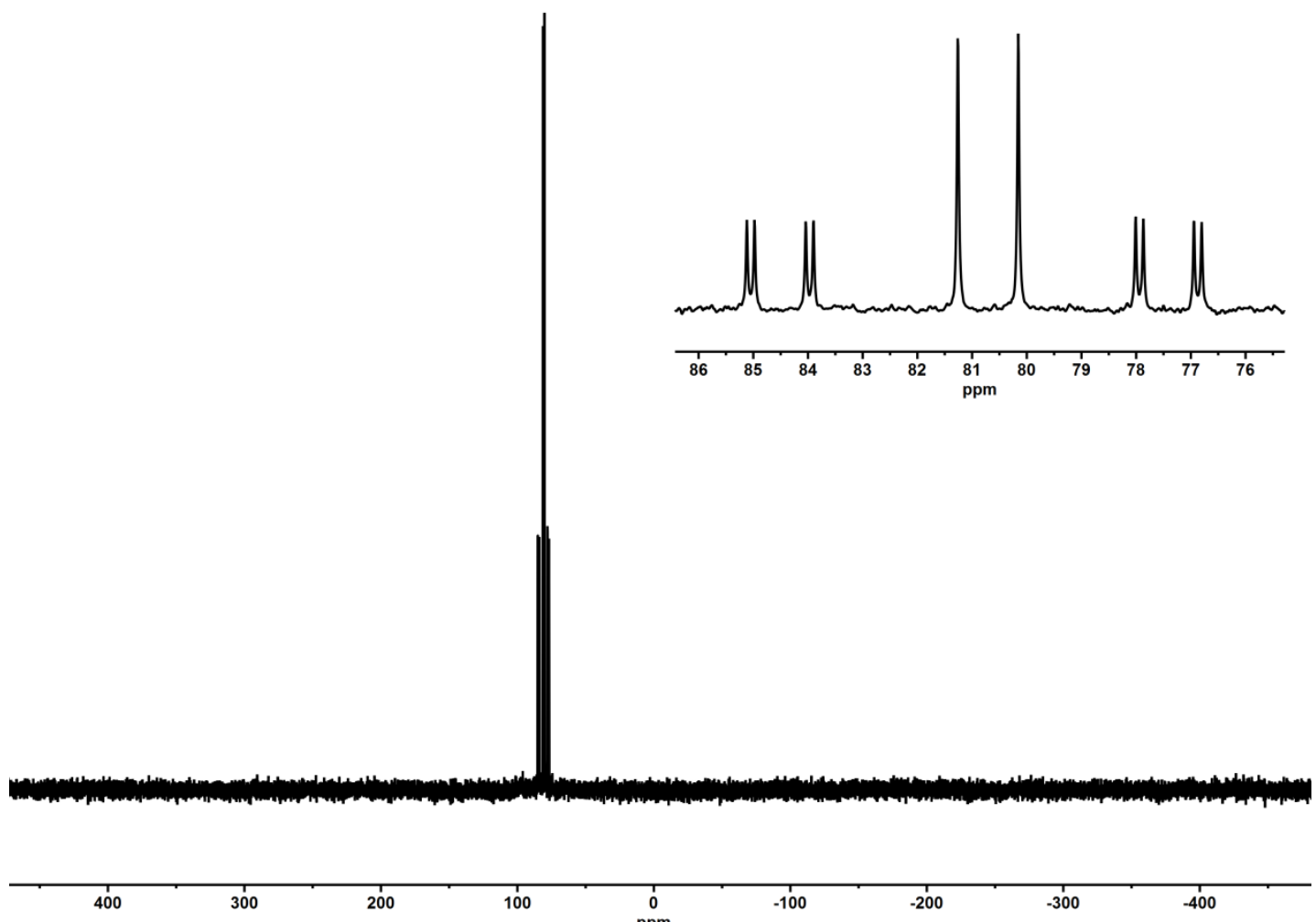

Figure S24: The solution ${ }^{31} \mathrm{P}\left\{{ }^{1} \mathrm{H}\right\} \mathrm{NMR}$ spm spectrum $\left(\mathrm{CD}_{2} \mathrm{Cl}_{2}, 298 \mathrm{~K}, 162 \mathrm{MHz}\right)$ of [1-hexadiene][BArF ${ }_{4}$ ]. The inset is an enlargement of the resonances between $\delta 86$ and 76 . 


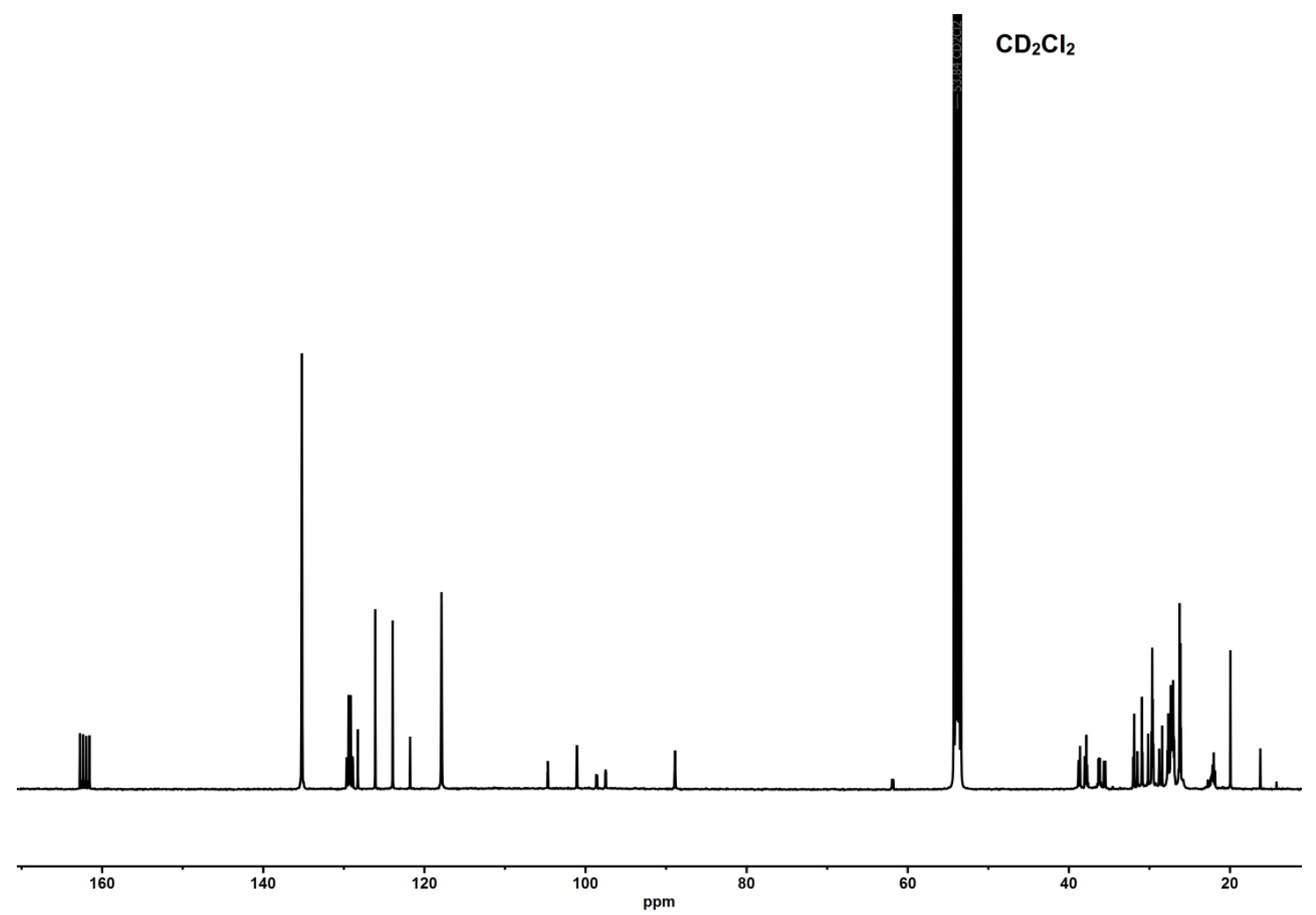

Figure S25: The solution ${ }^{13} \mathrm{C}\left\{{ }^{1} \mathrm{H}\right\}$ NMR spectrum $\left(\mathrm{CD}_{2} \mathrm{Cl}_{2}, 298 \mathrm{~K}, 126 \mathrm{MHz}\right)$ of [1-hexadiene][BAr ${ }_{4}$ ]. 


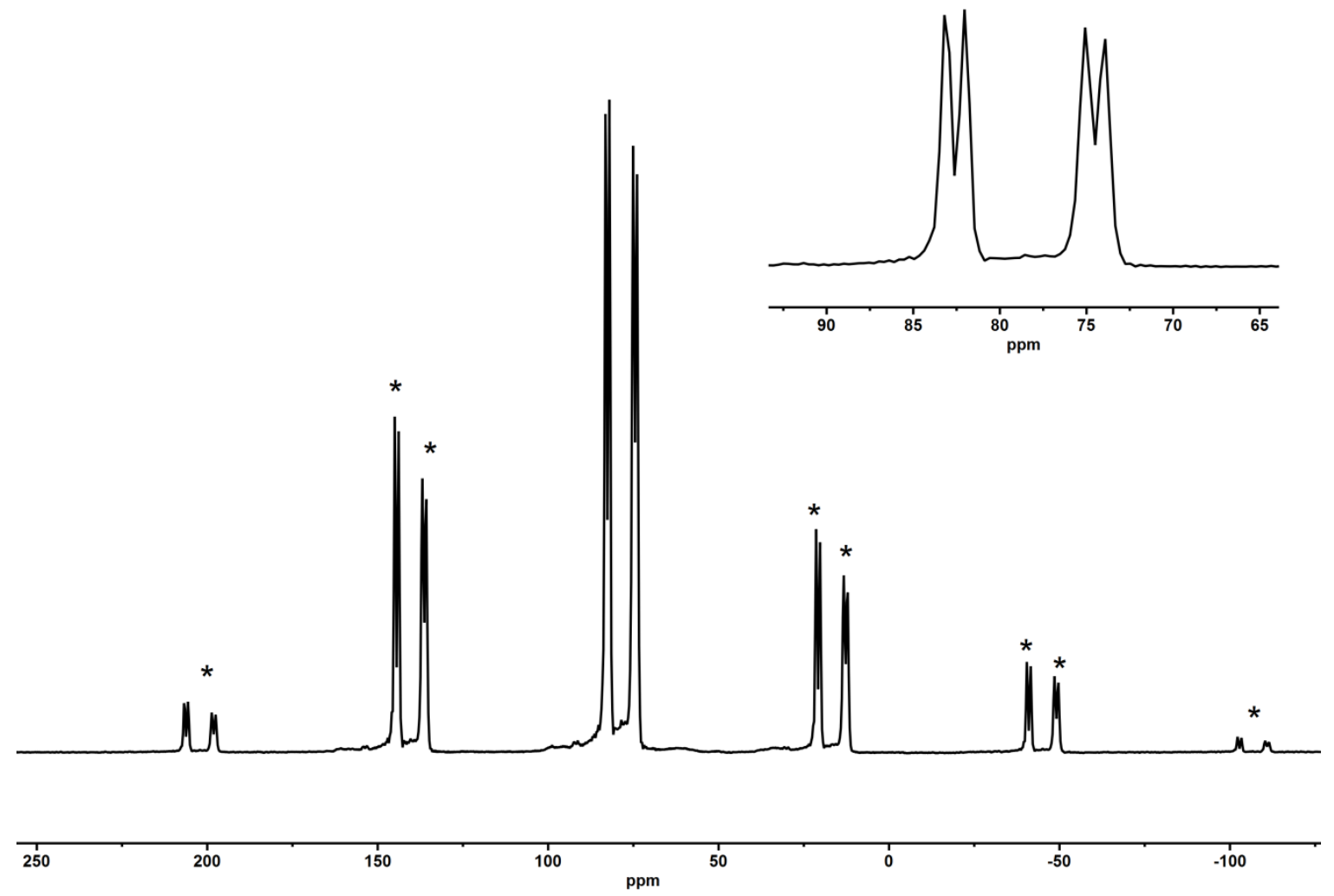

Figure S26: The ${ }^{31} \mathrm{P}\left\{{ }^{1} \mathrm{H}\right\}$ SSNMR spectrum (162 MHz, $294 \mathrm{~K}, 10 \mathrm{kHz}$ spin rate) of [1-hexadiene][BAr ${ }_{4}$ ].The resonances marked * are due to spinning sidebands

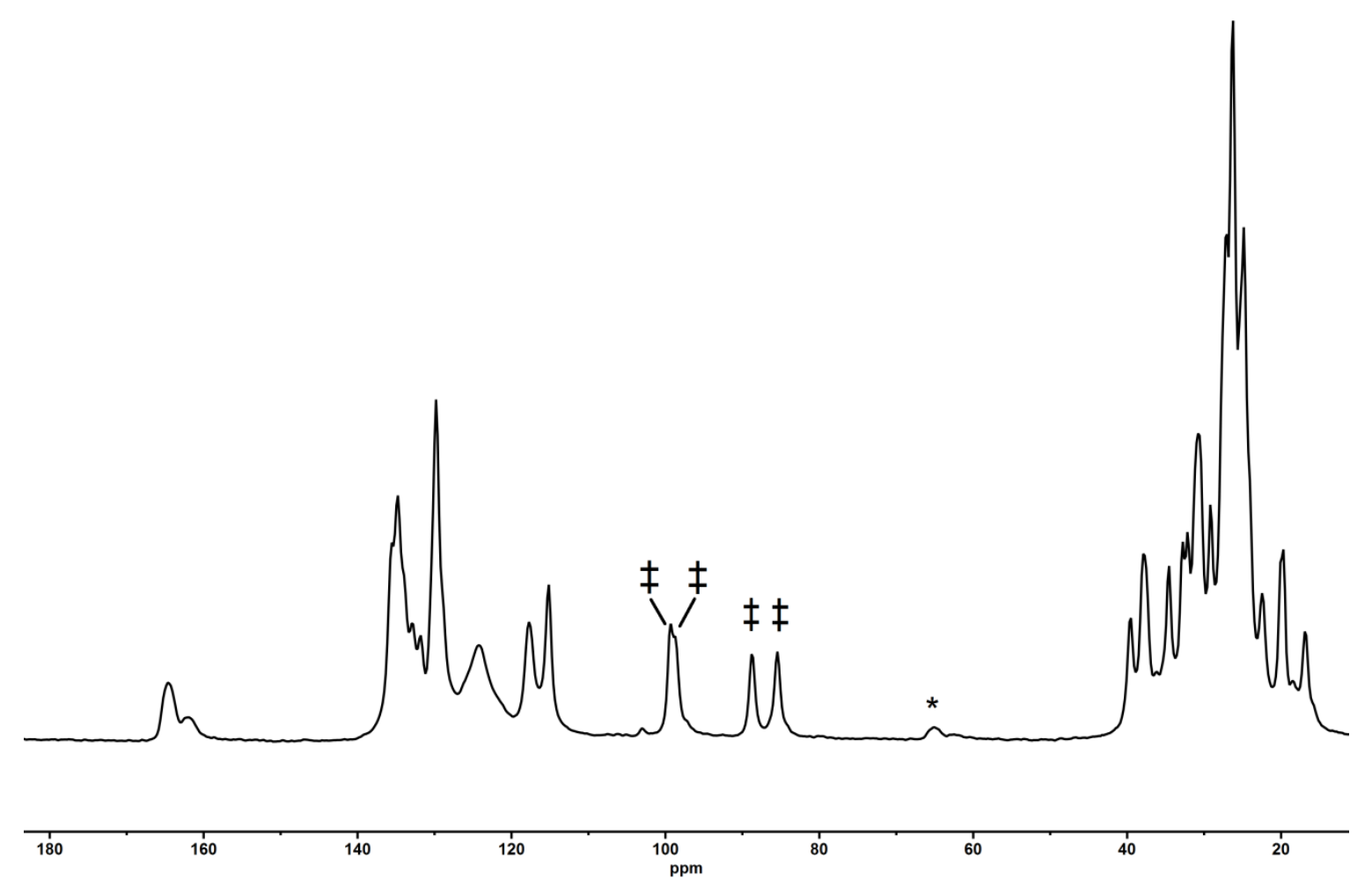

Figure S27: The ${ }^{13} \mathrm{C}\left\{{ }^{1} \mathrm{H}\right\}$ SSNMR spectrum (101 MHz, $294 \mathrm{~K}, 10 \mathrm{kHz}$ spin rate) of [1-hexadiene][BAr ${ }_{4}$ ]. The resonances marked * are due to spinning sidebands. The alkene resonances have been assigned $\neq$. 


\section{S.2.4.3. Synthesis of a hexane complex, [1-hexane][BAr$\left.{ }_{4}\right]$}

For Single-Crystal X-Ray Diffraction: Ruby red crystals of [1-hexadiene][BAr ${ }_{4}$ ] (1.5 mgs) were placed in a J. Young NMR tube and treated with $\mathrm{H}_{2}$ (1 bar, $298 \mathrm{~K}, 7$ minutes). The colour of the crystals slowly turned a dark red over this time. The crystalline material was then removed from the tube and coated with Fomblin ${ }^{\circledR} Y$ oil under an argon flush. A suitable crystal was then selected and transferred to the cryostream of a diffractometer $(150 \mathrm{~K})$. An $X$-ray diffraction study was undertaken and the structure of $\left[\mathrm{Rh}\left(\mathrm{Cy}_{2} \mathrm{PCH}_{2} \mathrm{CH}_{2} \mathrm{PCy}\right)\left(\mathrm{C}_{6} \mathrm{H}_{14}\right)\right]\left[\mathrm{BAr}_{4}\right]$ [1-hexane][BAr $\left.{ }_{4}\right]$ was refined. For further details of structural refinement please refer to Section S.3.

For Solid-State NMR Spectroscopy: A powdered microcrystalline sample of [1-hexadiene][BAr ${ }_{4}$ ] (45 mgs) was packed in a $4.0 \mathrm{~mm}$ SSNMR rotor, inside an argon filled glove box. The rotor was then placed in a custom-built glass $\mathrm{J}$. Young flask ${ }^{\mathrm{S5}}$ and the sample was then exposed to $\mathrm{H}_{2}(1$ bar, $298 \mathrm{~K})$. After 7 minutes, the rotor cap was fitted under a flush of $\mathrm{H}_{2}$. The sample was rapidly transferred to the bore of a SSNMR spectrometer, cooled to $158 \mathrm{~K}\left(\sim 10\right.$ minutes) and analysed by ${ }^{31} \mathrm{P}\left\{{ }^{1} \mathrm{H}\right\}$ and ${ }^{13} \mathrm{C}\left\{{ }^{1} \mathrm{H}\right\}$ solid-state NMR spectroscopy.

Note: These conditions were optimised to form [1-hexane][BAr${ }_{4}$ ]. At $158 \mathrm{~K}$, no onward reactivity with $\mathrm{H}_{2}$ is observed. Further exposure to $\mathrm{H}_{2}$ (30 minutes) at $298 \mathrm{~K}$ results in complete decomposition to $\left[1-\mathrm{BAr}_{4}{ }^{\mathrm{S}}\right.$. $^{\mathrm{S}}$

\section{S.2.4.4. Charaterisation data and spectra for [1-hexane][BAr $\left.{ }_{4}\right]$}

${ }^{31} \mathrm{P}\left\{{ }^{1} \mathrm{H}\right\}$ SSNMR (162 MHz, $158 \mathrm{~K}, 10 \mathrm{kHz}$ spin rate): $\delta 106.0\left(\mathrm{~d}, J_{\mathrm{RhP}}=226 \mathrm{~Hz}\right), 102.5 .(\mathrm{d}$, $J_{\mathrm{RhP}}=216 \mathrm{~Hz}$ ).

${ }^{13} \mathrm{C}\left\{{ }^{1} \mathrm{H}\right\}$ SSNMR (101 MHz, $158 \mathrm{~K}, 10 \mathrm{kHz}$ spin rate): $\delta 162.7(\operatorname{ArC}), 134.0(\operatorname{ArC}), 129.1$ (ArC), 124.0 (ArC), 115.7 (ArC), 37.2, 34.9, 34.6, 32.5, 31.5, 30.7 , 28.1, 24.8, 22.6, 20.1, $18.1,13.9,13.1$ (multiple aliphatic resonances). 

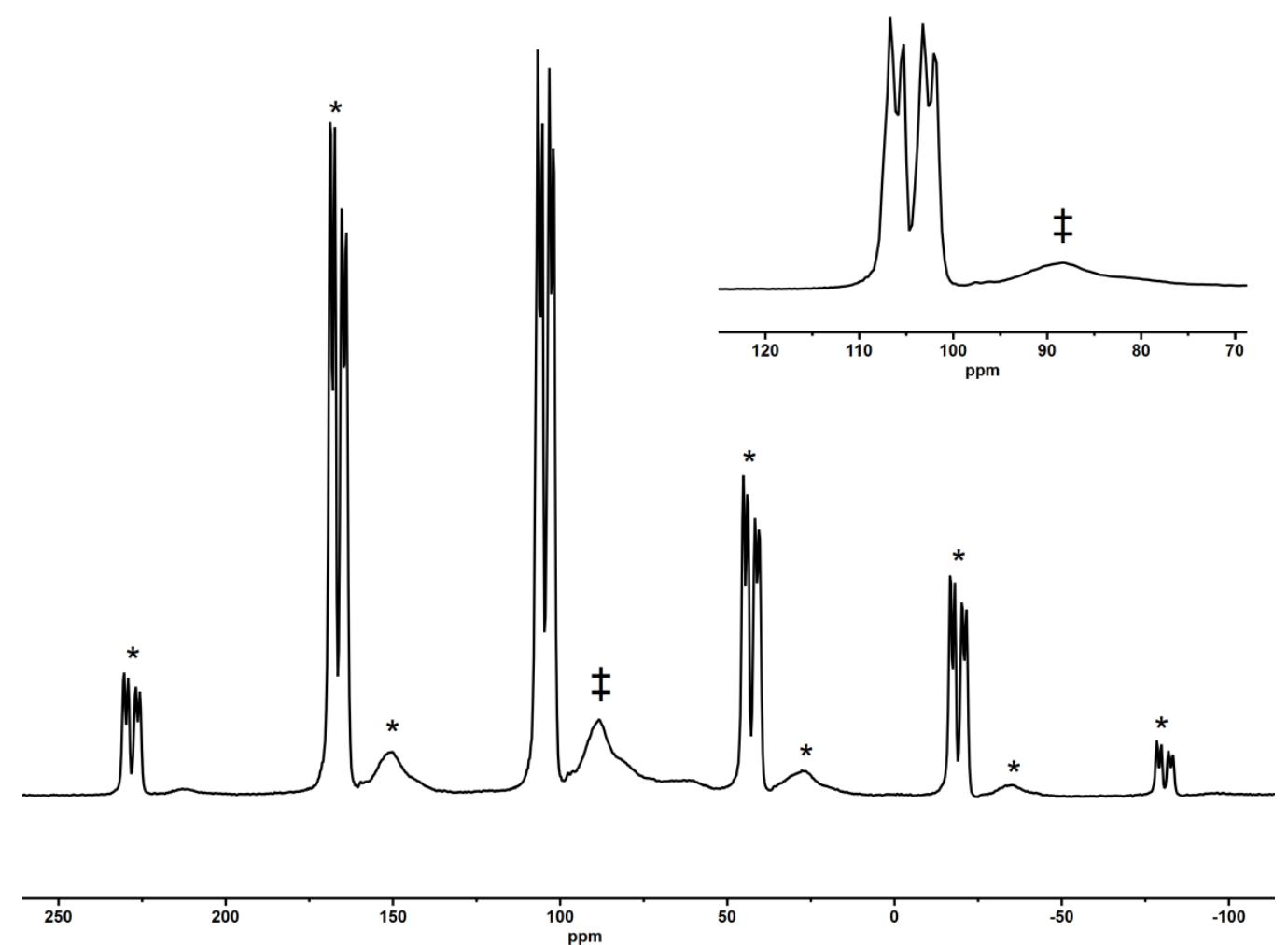

Figure S28: The ${ }^{31} \mathrm{P}\left\{{ }^{1} \mathrm{H}\right\}$ SSNMR spectrum (162 MHz, $158 \mathrm{~K}, 10 \mathrm{kHz}$ spin rate) [1-hexane][BAr ${ }_{4}$ ] prepared in situ after 15 minutes under $\mathrm{H}_{2}$ at 298K. The resonances marked are assigned to an $\left[1-\mathrm{BAr}_{4}\right]^{\mathrm{S} 2}$ decomposition product under $\mathrm{H}_{2}$ at $298 \mathrm{~K}$. The resonances marked * are due to spinning sidebands. The inset is an enlargement of the central resonances.

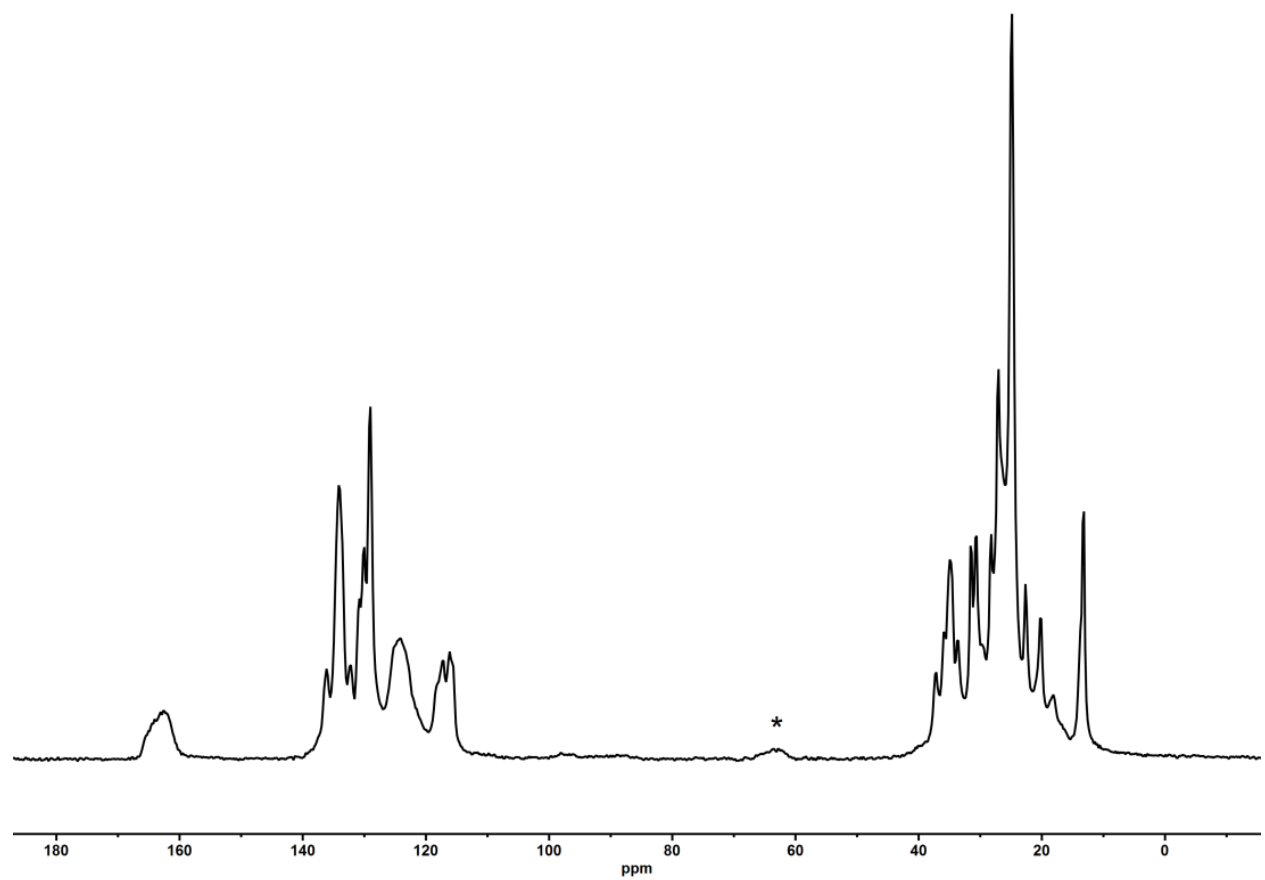

Figure S29: The ${ }^{13} \mathrm{C}\left\{{ }^{1} \mathrm{H}\right\}$ SSNMR spectrum $\left(100 \mathrm{MHz}, 158 \mathrm{~K}\right.$, under $\mathrm{H}_{2}, 10 \mathrm{kHz}$ spin rate) of [1-hexane][BArF $\left.{ }_{4}\right]$ prepared in situ. The resonance marked * is due to a spinning sideband. 


\section{S.2.4.5. Stability of [1-hexane][BArF $\left.{ }_{4}\right]$ at $294 \mathrm{~K}$}

On the same sample as presented in Figures S31 to S32, the solid-state NMR spectrometer was warmed to $294 \mathrm{~K}$ and ${ }^{31} \mathrm{P}\left\{{ }^{1} \mathrm{H}\right\}$ SSNMR spectra collected until complete decomposition of [1-hexane][BAr ${ }_{4}$ ] was observed. During this time, the solid-state rotor was under a flushing $\mathrm{N}_{2}$ atmosphere.

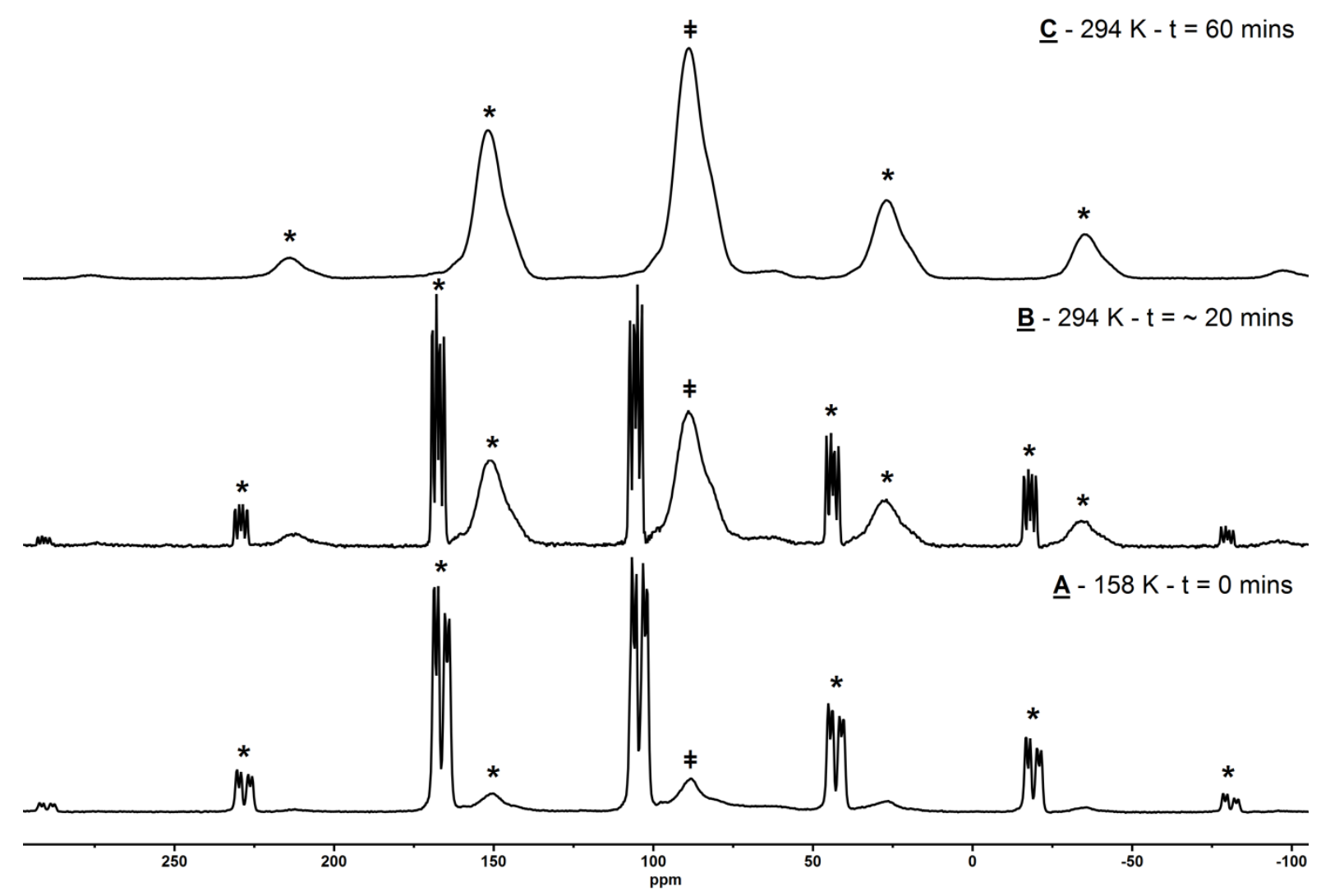

Figure S30: The ${ }^{31} \mathrm{P}\left\{{ }^{1} \mathrm{H}\right\}$ SSNMR (162 MHz, $10 \mathrm{kHz}$ spin rate) spectra of $\mathbf{A}$ : [1-hexane][BAr ${ }_{4}$ ] as prepared at $158 \mathrm{~K}$. B: Spectrum collected upon warming to $294 \mathrm{~K}$. Total time at $294 \mathrm{~K}$ including instrument warming, stabilisation and experimental set-up / collection is $\mathbf{2 0}$ minutes. C: Total time at $294 \mathrm{~K}$ including instrument warming, stabilisation and experimental set-up / collection is $\mathbf{6 0}$ minutes. Peaks marked * are spinning sidebands and $\neq$ are assigned to $\left[1-\mathrm{BAr}_{4}\right]^{\mathrm{S2}}$ in all parts. 


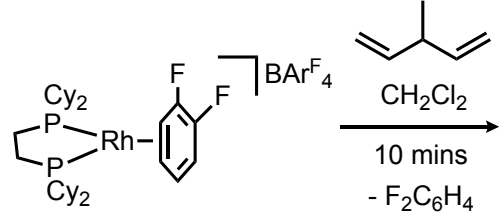

$\left[1-\mathrm{F}_{2} \mathrm{C}_{6} \mathrm{H}_{4}\right]\left[\mathrm{BAr}_{4}\right]$

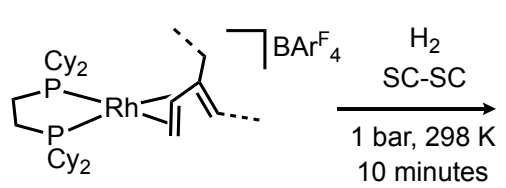

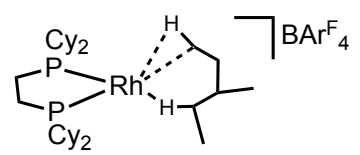

[1-(3-methylpentane)][BAr $\left.{ }_{4}\right]$

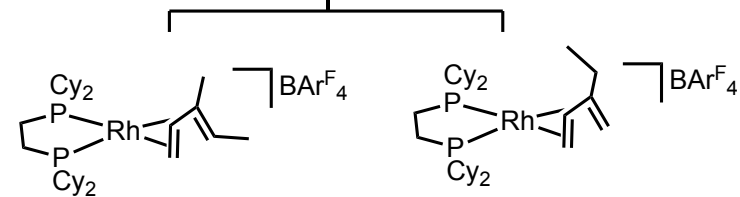

3-methyl-1,3-pentadiene

2-ethylbutadiene

$\left[1-\left(\mathrm{C}_{6} \mathrm{H}_{10}\right)\right]\left[\mathrm{BAr}_{4}\right]$

Isomers Present in both Solution and Solid-State

\section{S.2.5.1. Synthesis of a precursor 3-methylpentane complex, [1-( $\left.\left.\mathrm{C}_{6} \mathrm{H}_{10}\right)\right]\left[B A r^{\mathrm{F}}{ }_{4}\right]$} ( $\mathrm{C}_{6} \mathrm{H}_{10}=3$-methyl-1,3-pentadiene or 2-ethylbutadiene $)$

A pale-yellow solution of $\left[1-\mathbf{F}_{2} \mathbf{C}_{6} \mathrm{H}_{4}\right]\left[\mathrm{BAr}_{4}{ }_{4}\right](600 \mathrm{mgs}, 0.40 \mathrm{mmol})$ in $\mathrm{CH}_{2} \mathrm{Cl}_{2}(20 \mathrm{~mL})$ was treated with neat 3-methyl-1,4-pentadiene $(0.1 \mathrm{~mL}, 0.78 \mathrm{mmol})$ at ambient temperature. The resultant deep red solution was stirred at ambient temperature for 30 minutes. Pentane (100 $\mathrm{mL}$ ) was then added with vigorous stirring which resulted in the formation of a red precipitate. The solid was isolated by filtration and washed with pentane $(2 \times 20 \mathrm{~mL})$. The solid was then dissolved in $\mathrm{CH}_{2} \mathrm{Cl}_{2}(\sim 8 \mathrm{~mL})$ and filtered into a J. Young crystallisation flask and layered with pentane. Deep red crystals were obtained after storage for 1 week at room temperature of $\left[\mathrm{Rh}\left(\mathrm{Cy}_{2} \mathrm{PCH}_{2} \mathrm{CH}_{2} \mathrm{PCy}_{2}\right)\left(\mathrm{C}_{6} \mathrm{H}_{10}\right)\right]\left[\mathrm{BAr}_{4}{ }_{4}\right], \quad\left[1-\left(\mathrm{C}_{6} \mathrm{H}_{10}\right)\right]\left[\mathrm{BAr}_{4}{ }_{4}\right] \quad\left(\mathrm{C}_{6} \mathrm{H}_{10}=\right.$ 3-methyl-1,3-pentadiene or 2-ethylbutadiene) (Yield: 515 mgs, 88 \%). 


\section{S.2.5.2. Charaterisation data and spectra for $\left[1-\left(\mathrm{C}_{6} \mathrm{H}_{10}\right)\right]\left[B A r^{\mathrm{F}}{ }_{4}\right]$}

${ }^{1} \mathrm{H}$ NMR $\left(\mathrm{CD}_{2} \mathrm{Cl}_{2}, 298 \mathrm{~K}, 400 \mathrm{MHz}\right): \delta 7.72$ (s, 8H, ortho-ArH), 7.56 (s, 4H, para-ArH), 5.16 (d. of d. of d., alkene- $\mathrm{H}, J=13.9,8.6,2.2 \mathrm{~Hz}$ ), 5.01 (d. of d. of d., alkene- $\mathrm{H}, J=13.7,8.1,2.1$ $\mathrm{Hz}$ ), 4.37-4.33 (broad m., alkene-H), 4.04 (d. of d. of d., alkene-H, $J=8.2,4.3,1.4 \mathrm{~Hz}$ ), 3.90 (apparent quartet, alkene- $\mathrm{H}, \mathrm{J}=6.3 \mathrm{~Hz}$ ), 2.31 -1.51 (br m, overlapping aliphatic $\mathrm{CH}$ ), 1.440.82 (br m, aliphatic $\mathrm{CH}$ ).

${ }^{31} \mathrm{P}\left\{{ }^{1} \mathrm{H}\right\}$ NMR $\left(\mathrm{CD}_{2} \mathrm{Cl}_{2}, 298 \mathrm{~K}, 162 \mathrm{MHz}\right)$ : 3-methyl-1,3-pentadiene Isomer: $\delta 85.6$ (d.d., J $\mathrm{JhP}$ $=171 \mathrm{~Hz}, J_{\mathrm{PP}}=24 \mathrm{~Hz}$ ), 79.2 (d.d., $J_{\mathrm{RhP}}=169 \mathrm{~Hz}, J_{\mathrm{PP}}=24 \mathrm{~Hz}$ ). 2-ethylbutadiene Isomer: $\delta$ 84.0 (d.d., $J_{\mathrm{RhP}}=172 \mathrm{~Hz}, J_{\mathrm{PP}}=23 \mathrm{~Hz}$ ), 75.3 (d.d., $J_{\mathrm{RhP}}=163 \mathrm{~Hz}, J_{\mathrm{PP}}=23 \mathrm{~Hz}$ ).

${ }^{19} \mathrm{~F}\left\{{ }^{1} \mathrm{H}\right\}$ NMR $\left(\mathrm{CD}_{2} \mathrm{Cl}_{2}, 298 \mathrm{~K}, 377 \mathrm{MHz}\right): \delta-62.9(\mathrm{~s})$.

${ }^{13} \mathrm{C}\left\{{ }^{1} \mathrm{H}\right\}$ NMR $\left(\mathrm{CD}_{2} \mathrm{Cl}_{2}, 298 \mathrm{~K}, 126 \mathrm{MHz}\right): \delta 162.7$ (q, J $\mathrm{CB}=50 \mathrm{~Hz}$, ipso-ArC), 134.7 (s, ortho$\operatorname{ArC}$ ), 128.8 (q, $J_{\mathrm{CF}}=32 \mathrm{~Hz}$, meta-ArC), 125.1 (apparent triplet, $\mathrm{C}=\mathrm{C}, J=4.3 \mathrm{~Hz}$ ), 124.6 (q, $J_{\mathrm{CF}}=272 \mathrm{~Hz},-\mathrm{CF}_{3}$ ), 119.3 (apparent triplet, $\mathrm{C}=\mathrm{C}, J=4.2 \mathrm{~Hz}$ ), 117.4 (s, para-ArC), 99.6 (apparent triplet, $\mathrm{C}=\mathrm{C}, J=5.5 \mathrm{~Hz}$ ), 98.4 (apparent triplet, $\mathrm{C}=\mathrm{C}, J=5.7 \mathrm{~Hz}$ ), 86.0 (d. of d., $\mathrm{C}=\mathrm{C}, J=12.8,3.3 \mathrm{~Hz}$ ), 63.4 (d. of d. of d., $\mathrm{C}=\mathrm{C}, J=10.5,7.8,1.5 \mathrm{~Hz}$ ), 62.8 (d. of d. of d., $\mathrm{C}=\mathrm{C}, J=14.2,5.4,1.6 \mathrm{~Hz}$ ), 59.2 (d. of d. of d., $\mathrm{C}=\mathrm{C}, J=11.8,8.2,2.1 \mathrm{~Hz}), 38.3-21.1(\mathrm{~m}$, overlapping cyclohexyl- $\mathrm{CH}_{2}$ from both isomers), 19.4, 15.9, 14.5, 13.8 (s, -ethyl/-methyl groups from co-ordinated isomers).

${ }^{31} \mathrm{P}\left\{{ }^{1} \mathrm{H}\right\}$ SSNMR (162 MHz, $294 \mathrm{~K}, 10 \mathrm{kHz}$ spin rate): $\delta 84.8$ (from two, coincident ${ }^{31} \mathrm{P}$ environments), 79.6, 74.9.

${ }^{13} \mathrm{C}\left\{{ }^{1} \mathrm{H}\right\}$ SSNMR (101 MHz, $294 \mathrm{~K}, 10 \mathrm{kHz}$ spin rate): $\delta 163.3$ (ipso-ArC), 134.7 (ortho-ArC), 129.5 (meta-ArC), 124.4 (br, $\left.\mathrm{CF}_{3}\right), 117.2$ (para-ArC), $101.8(\mathrm{C}=\mathrm{C}), 99.8(\mathrm{C}=\mathrm{C}), 84.3(\mathrm{C}=\mathrm{C})$, $63.5(\mathrm{C}=\mathrm{C}), 38.7(\mathrm{CH}), 36.0(\mathrm{CH}), 30.8\left(\mathrm{CH}_{2}\right), 26.2\left(\mathrm{CH}_{2}\right), 25.3\left(\mathrm{CH}_{2}\right), 21.2\left(\mathrm{CH}_{2}\right), 17.5$ $\left(\mathrm{CH}_{2}\right), 14.5\left(\mathrm{CH}_{2}\right)$.

ESI-MS (calc. for $\left.\left[\mathrm{Rh}\left(\mathrm{Cy}_{2} \mathrm{PCH}_{2} \mathrm{CH}_{2} \mathrm{PCy}_{2}\right)\left(\mathrm{C}_{6} \mathrm{H}_{10}\right)\right]^{+}\right): m / z 607.3097$ (607.3063).

Elemental Analysis (calc. for $\mathrm{C}_{64} \mathrm{H}_{70} \mathrm{BF}_{24} \mathrm{P}_{2} \mathrm{Rh}$ ): C 52.38 (52.26), H 4.57 (4.80). 


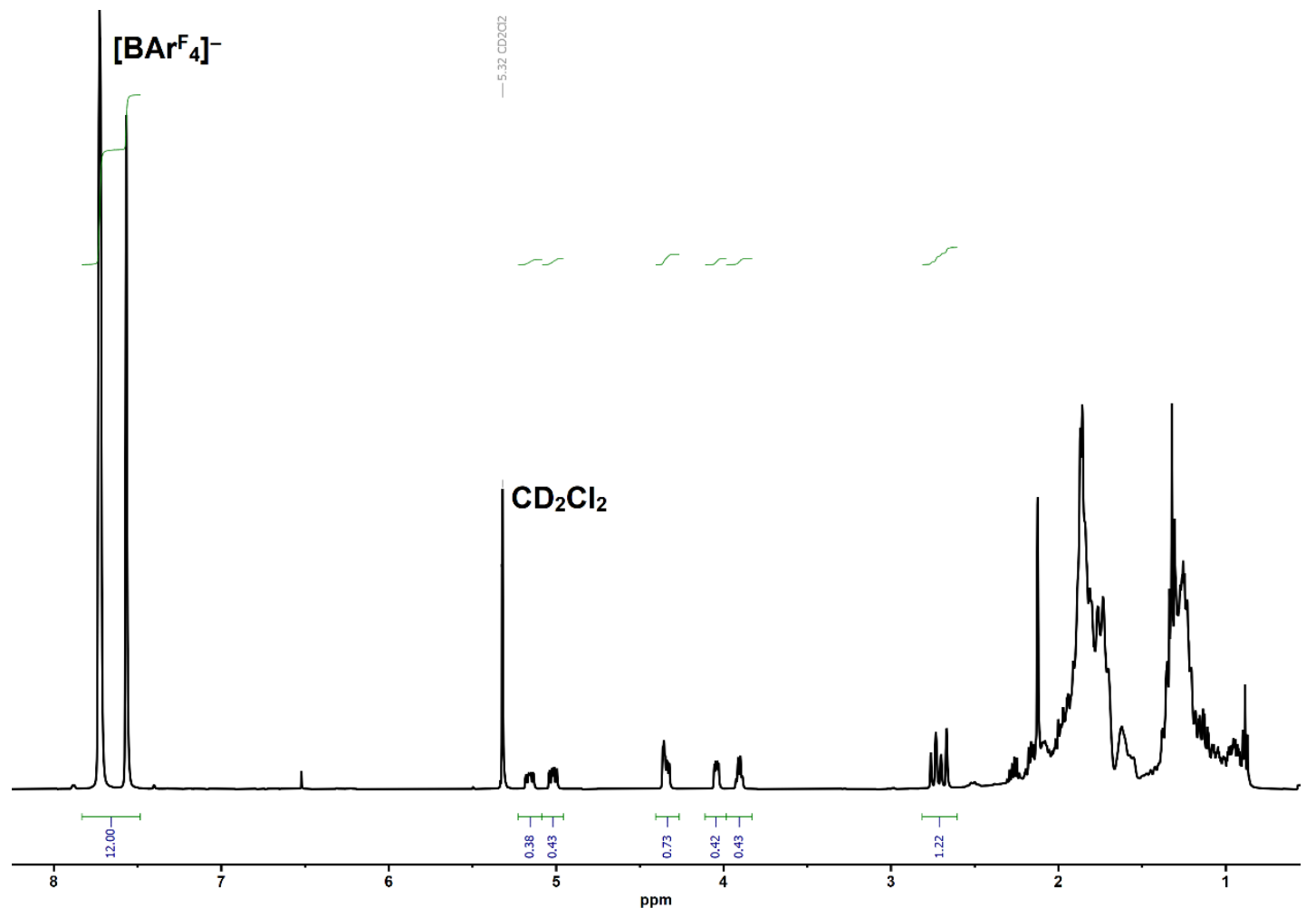

Figure S31: The solution ${ }^{1} \mathrm{H}$ NMR spectrum $\left(\mathrm{CD}_{2} \mathrm{Cl}_{2}, 298 \mathrm{~K}, 400 \mathrm{MHz}\right)$ of $\left[1-\left(\mathrm{C}_{6} \mathrm{H}_{10}\right)\right]\left[\mathrm{BAr}_{4}{ }_{4}\right]$ $\left(\mathrm{C}_{6} \mathrm{H}_{10}=3\right.$-methyl-1,3-pentadiene or 2-ethylbutadiene $)$.

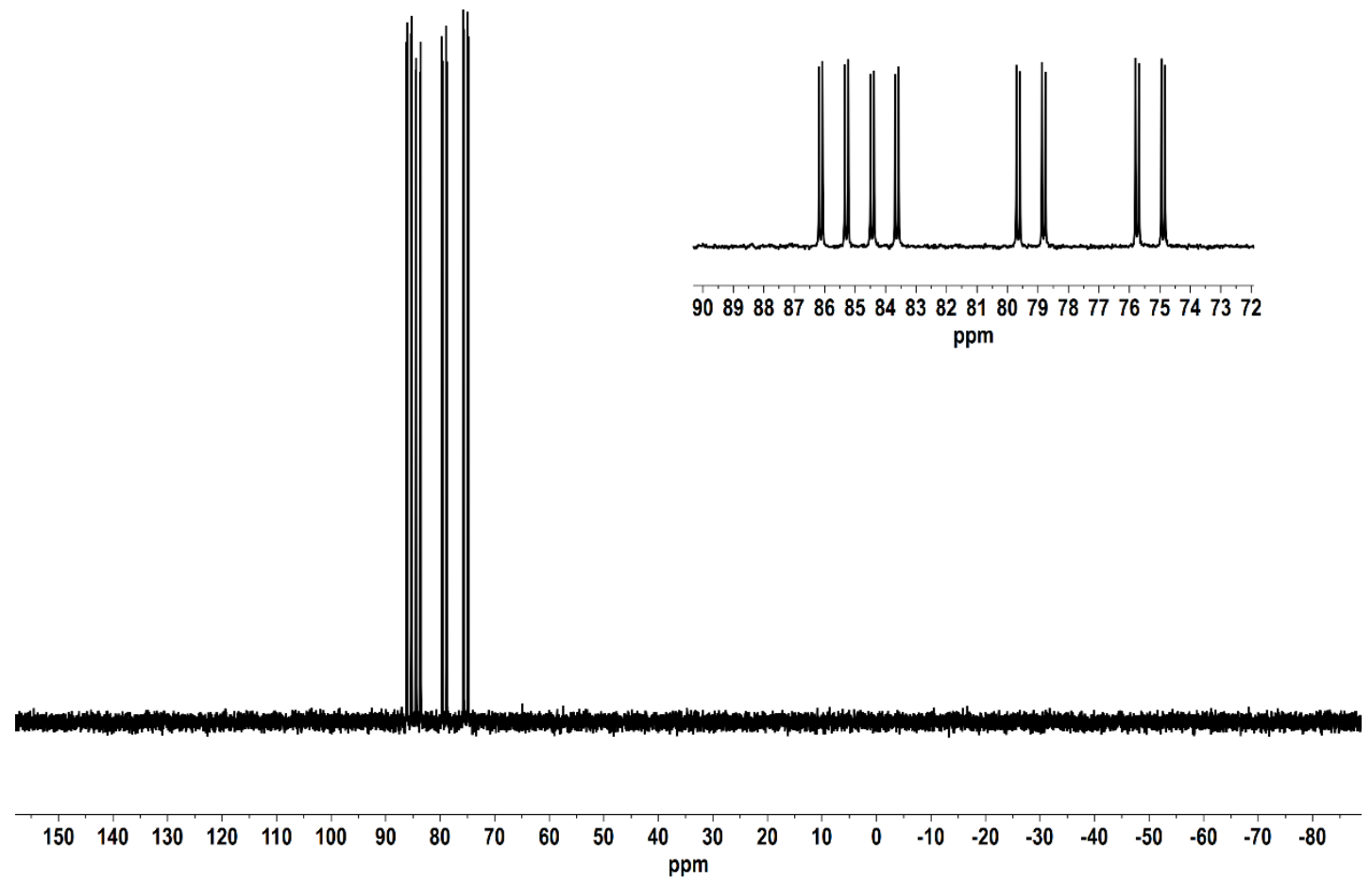

Figure S32: The solution ${ }^{31} \mathrm{P}\left\{{ }^{1} \mathrm{H}\right\}$ NMR spectrum $\left(\mathrm{CD}_{2} \mathrm{Cl}_{2}, 298 \mathrm{~K}, 162 \mathrm{MHz}\right)$ of [1-( $\left.\left.\mathrm{C}_{6} \mathrm{H}_{10}\right)\right]\left[\mathrm{BAr}_{4}{ }_{4}\right]\left(\mathrm{C}_{6} \mathrm{H}_{10}=3\right.$-methyl-1,3-pentadiene or 2-ethylbutadiene). The inset is an enlargement of the resonances between $\delta 90$ and 72 . 


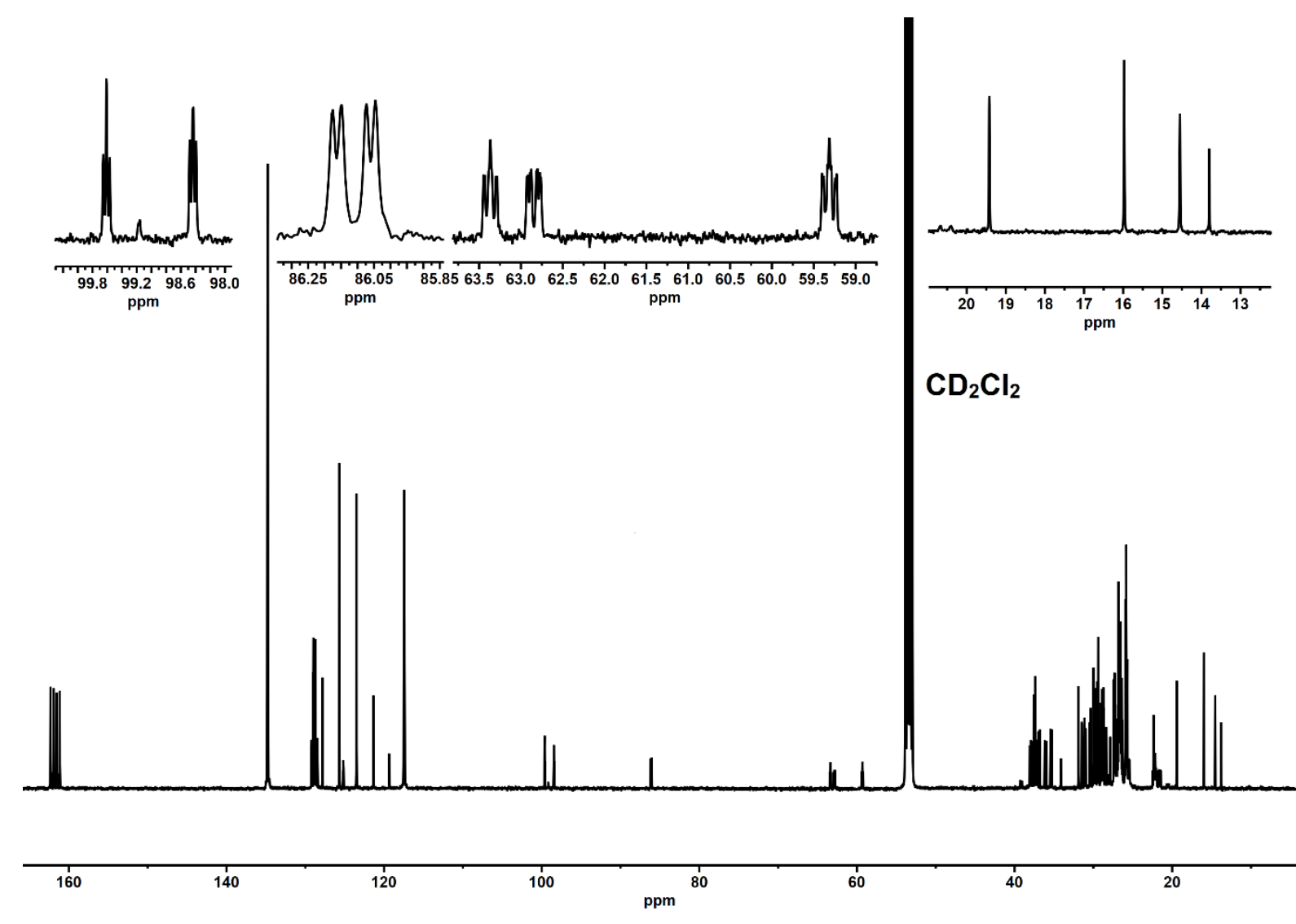

Figure S33: The solution ${ }^{13} \mathrm{C}\left\{{ }^{1} \mathrm{H}\right\}$ NMR spectrum $\left(\mathrm{CD}_{2} \mathrm{Cl}_{2}, 298 \mathrm{~K}, 126 \mathrm{MHz}\right)$ of [1-( $\left.\left.\mathrm{C}_{6} \mathrm{H}_{10}\right)\right]\left[\mathrm{BAr}_{4}{ }_{4}\right]\left(\mathrm{C}_{6} \mathrm{H}_{10}=3\right.$-methyl-1,3-pentadiene or 2-ethylbutadiene $)$. The insets are enlargements of the resonances of alkene or ethyl/-methyl groups from co-ordinated the $\mathrm{C}_{6}$ isomers. 


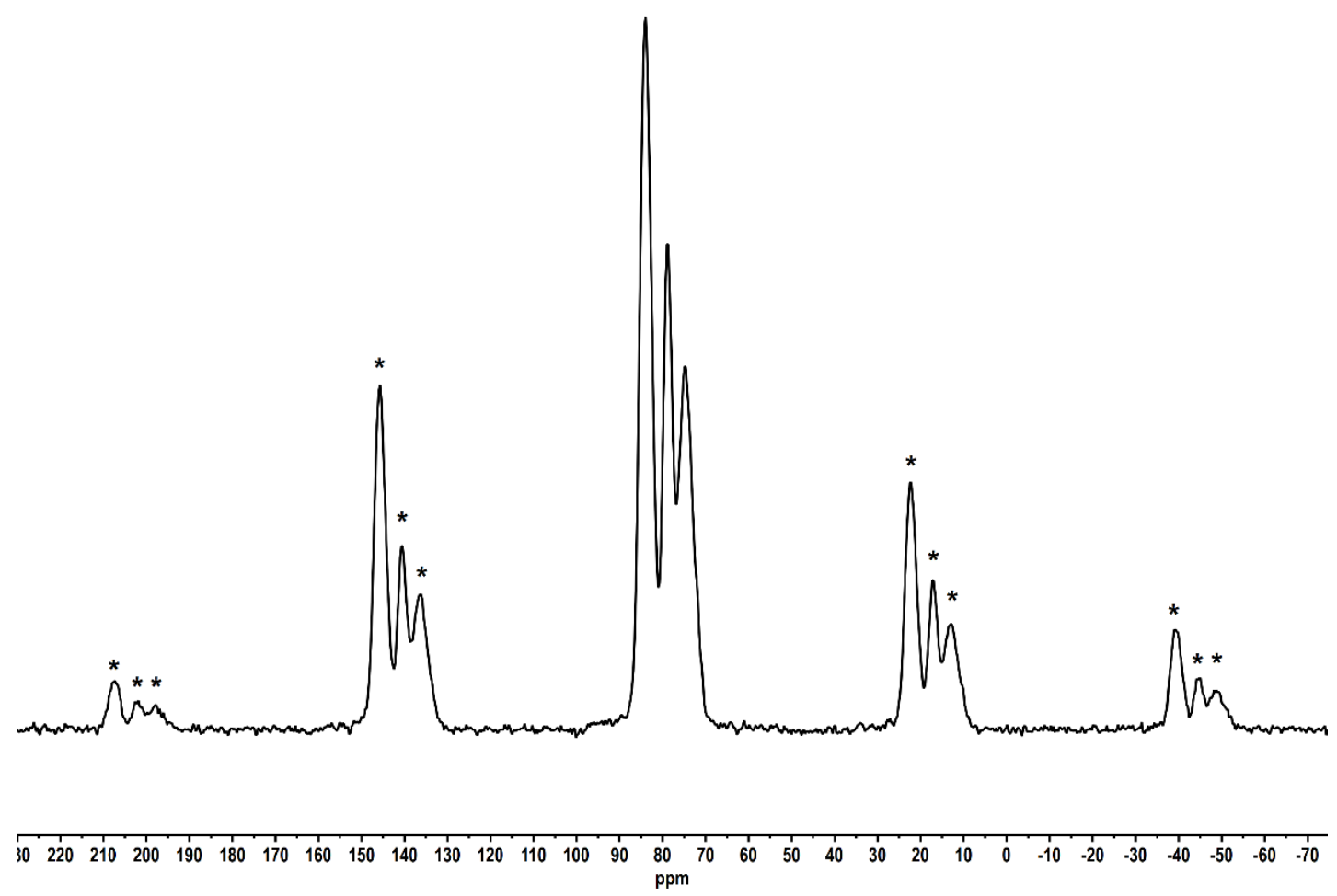

Figure S34: The ${ }^{31} \mathrm{P}\left\{{ }^{1} \mathrm{H}\right\}$ SSNMR spectrum (162 MHz, $294 \mathrm{~K}, 10 \mathrm{kHz}$ spin rate) of [1-( $\left.\left.\mathrm{C}_{6} \mathrm{H}_{10}\right)\right]\left[\mathrm{BAr}_{4}{ }_{4}\right]\left(\mathrm{C}_{6} \mathrm{H}_{10}=3\right.$-methyl-1,3-pentadiene or 2-ethylbutadiene). The resonances marked ${ }^{*}$ are due to spinning sidebands.

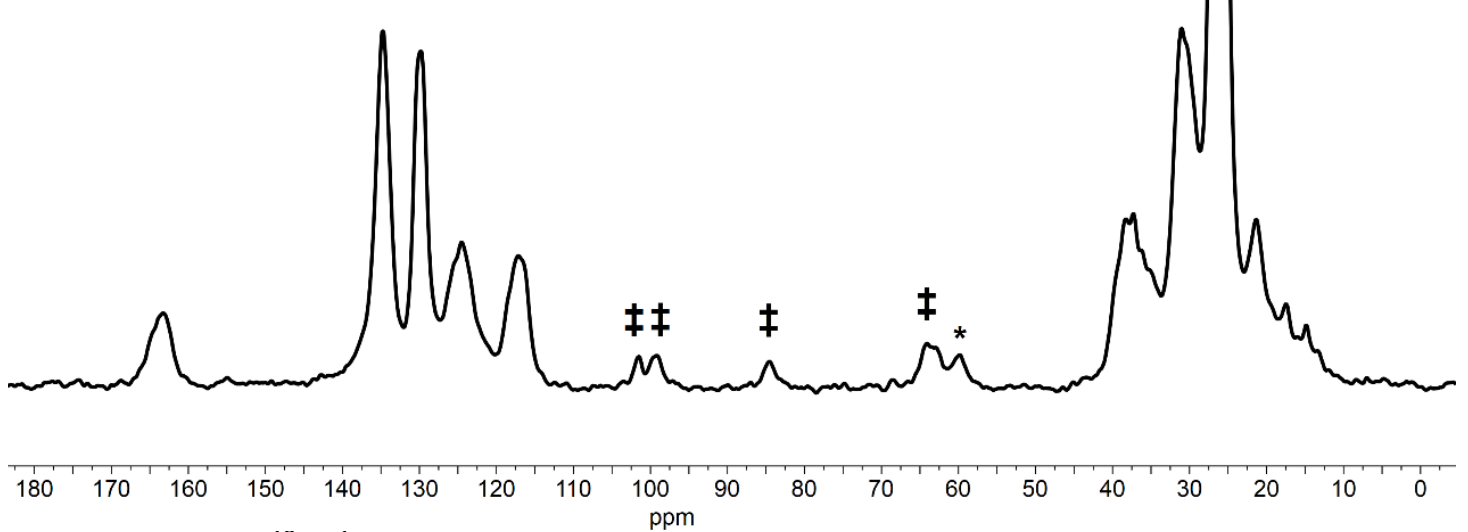

Figure S35: The ${ }^{13} \mathrm{C}\left\{{ }^{1} \mathrm{H}\right\}$ SSNMR spectrum (101 MHz, $294 \mathrm{~K}, 10 \mathrm{kHz}$ spin rate) of [1-( $\left.\left.\mathrm{C}_{6} \mathrm{H}_{10}\right)\right]\left[\mathrm{BAr}_{4}{ }_{4}\right]$. The resonances marked ${ }^{*}$ are due to spinning sidebands. The alkene resonances have been assigned $\neq$. 
S.2.5.3. Liberation of bound $C_{6}$-isomers from $\left[1-\left(\mathrm{C}_{6} \mathrm{H}_{10}\right)\right]\left[B \mathrm{Brr}_{4}^{\mathrm{F}}\right]\left(\mathrm{C}_{6} \mathrm{H}_{10}=\right.$

\section{3-methyl-1,3-pentadiene or 2-ethylbutadiene)}

A J. Young NMR tube was charged with crystalline sample of [1-( $\left.\left.\mathrm{C}_{6} \mathrm{H}_{10}\right)\right]\left[B A \mathrm{Br}_{4}\right]$ (30 mgs) dissolved in $\mathrm{CD}_{2} \mathrm{Cl}_{2}\left(0.5 \mathrm{~mL}\right.$, dark red solution) and $\mathrm{MeCN}-d_{3}(0.05 \mathrm{~mL})$ added to liberate the bound diene ligands. After 2 minutes, the solution had turned a pale-yellow colour which was confirmed to be $\left[\mathrm{Rh}\left(\mathrm{Cy}_{2} \mathrm{PCH}_{2} \mathrm{CH}_{2} \mathrm{PCy}\right)(\mathrm{MeCN})_{2}\right]\left[\mathrm{BAr}_{4}\right]$ by solution ${ }^{31} \mathrm{P}\left\{{ }^{1} \mathrm{H}\right\} \quad \mathrm{NMR}$ spectroscopy $\left(\delta 91, \mathrm{~d}, J_{\mathrm{RhP}}=175 \mathrm{~Hz}\right) .{ }^{\mathrm{S} 5}$ The volatile components were then isolated by trapto-trap distillation and analysed by solution ${ }^{1} \mathrm{H}$ NMR spectroscopy (Figure S39). A second batch was prepared in a similar fashion, however using neat $\mathrm{MeCN}$, and analysed by solution ${ }^{13} \mathrm{C}\left\{{ }^{1} \mathrm{H}\right\}$ NMR spectroscopy (Figure S38).

${ }^{13} \mathrm{C}\left\{{ }^{1} \mathrm{H}\right\}$ NMR $\left(\mathrm{CD}_{2} \mathrm{Cl}_{2}, 298 \mathrm{~K}, 126 \mathrm{MHz}\right): \delta 148.2,141.5,139.0,134.9,133.4,133.0,127.3$, $125.2,114.4,122.9,122.8,109.9,23.8,19.0,13.0,12.1,11.2,10.5$.

${ }^{1} \mathrm{H}$ NMR $\left(\mathrm{CD}_{2} \mathrm{Cl}_{2}, 298 \mathrm{~K}, 400 \mathrm{MHz}\right.$ ): $\delta 6.84$ (d. of d., $J=17.28,10.55 \mathrm{~Hz}$ ), 6.41 (d. of t., $J=$ 18.18, $10.70 \mathrm{~Hz}$ ), 5.60 (broad quartet, $J=6.46 \mathrm{~Hz}, \mathrm{H}_{4}$ ), 5.49 (broad quartet, $J=6.43 \mathrm{~Hz}, \mathrm{H}_{4}$ ), 5.26 (d., $J=18.6 \mathrm{~Hz}, H_{4}$ ), 5.21 (d., $J=17.6 \mathrm{~Hz}$ ), 5.08 (multiple overlapping signals), 5.04 (m.), 5.01 (m.), 4.92 (d., $J=11.85 \mathrm{~Hz}$ ), 2.26 (quartet, $J=7.70 \mathrm{~Hz}, \mathrm{H}_{5}$ ), 1.82 (m.), 1.75 (m.), 1.12 (t., $J=7.70 \mathrm{~Hz}$ ).

Literature Values: 2-ethylbutadiene ${ }^{\mathrm{s9}}$

${ }^{13} \mathrm{C}\left\{{ }^{1} \mathrm{H}\right\}$ NMR (Neat, $298 \mathrm{~K}, 10 \mathrm{MHz}$ ): $\delta 112.9$ [1], 148.3 [2], 139.6 [3], 114.7 [4], 24.6 [5], $12.6[6]$.

Literature Values: $(E)-3-$ methyl-1,3-pentadiene ${ }^{\mathrm{S10}}$

${ }^{13} \mathrm{C}\left\{{ }^{1} \mathrm{H}\right\}$ NMR $\left(\mathrm{CDCl}_{3}, 298 \mathrm{~K}, 15.1 \mathrm{MHz}\right): \delta 133.5$ [1], 133.2 [2], 125.0 [3], 113.0 [4], 19.71 [5], 12.9 [6].

Literature Values: (Z)-3-methyl-1,3-pentadiene ${ }^{\mathrm{S} 11}$

${ }^{13} \mathrm{C}\left\{{ }^{1} \mathrm{H}\right\}$ NMR $\left(\mathrm{CDCl}_{3}, 298 \mathrm{~K}, \mathbf{1 5 . 1} \mathrm{MHz}\right): \delta 141.6$ [1], 135.0 [2], 127.0 [3], 110.0 [4], 13.7 [5], $11.3[6]$.

Note: The solid-state structure of $\left[1-\left(\mathrm{C}_{6} \mathrm{H}_{10}\right)\right]\left[\mathrm{BAr}_{4}{ }_{4}\right]$ (see Figure S60) was modelled to include both 2-ethylbutadiene and (Z)-3-methyl-1,3-pentadiene. (E)-3-methyl-1,3-pentadiene could not be modelled in the solid-state yet is observed in the ${ }^{1} \mathrm{H}$ NMR spectrum of the isolated dienes. We suggest a fast isomerisation event during the liberation of the bound dienes. 


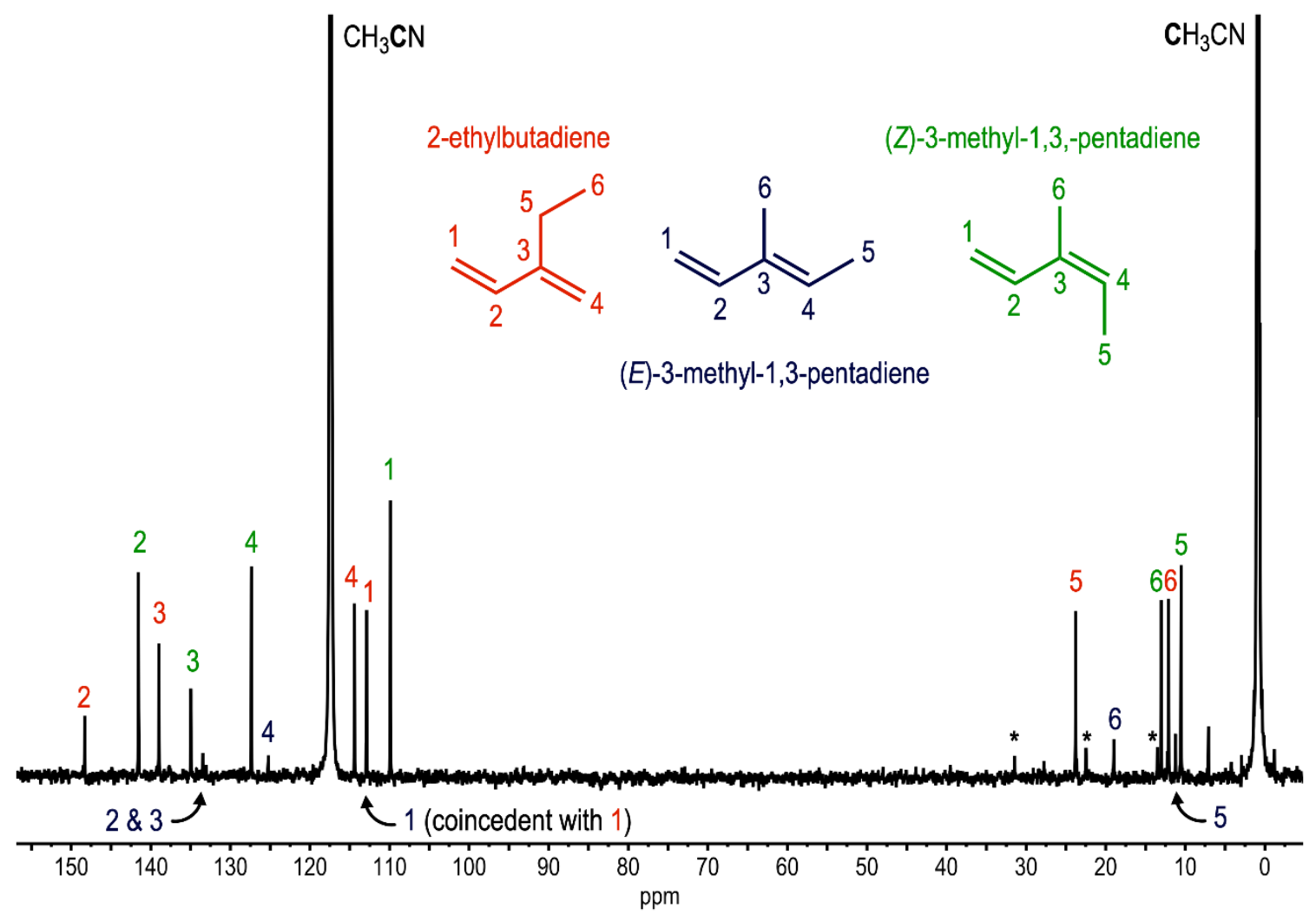

Figure S36: The solution ${ }^{13} \mathrm{C}\left\{{ }^{1} \mathrm{H}\right\}$ NMR $(\mathrm{MeCN}, 298 \mathrm{~K}, 126 \mathrm{MHz})$ spectrum of $\mathrm{C}_{6} \mathrm{H}_{10}$ volatiles. ${ }^{*}=n-$ hexane impurity.

(Z)-3-methyl-1,3,-pentadiene

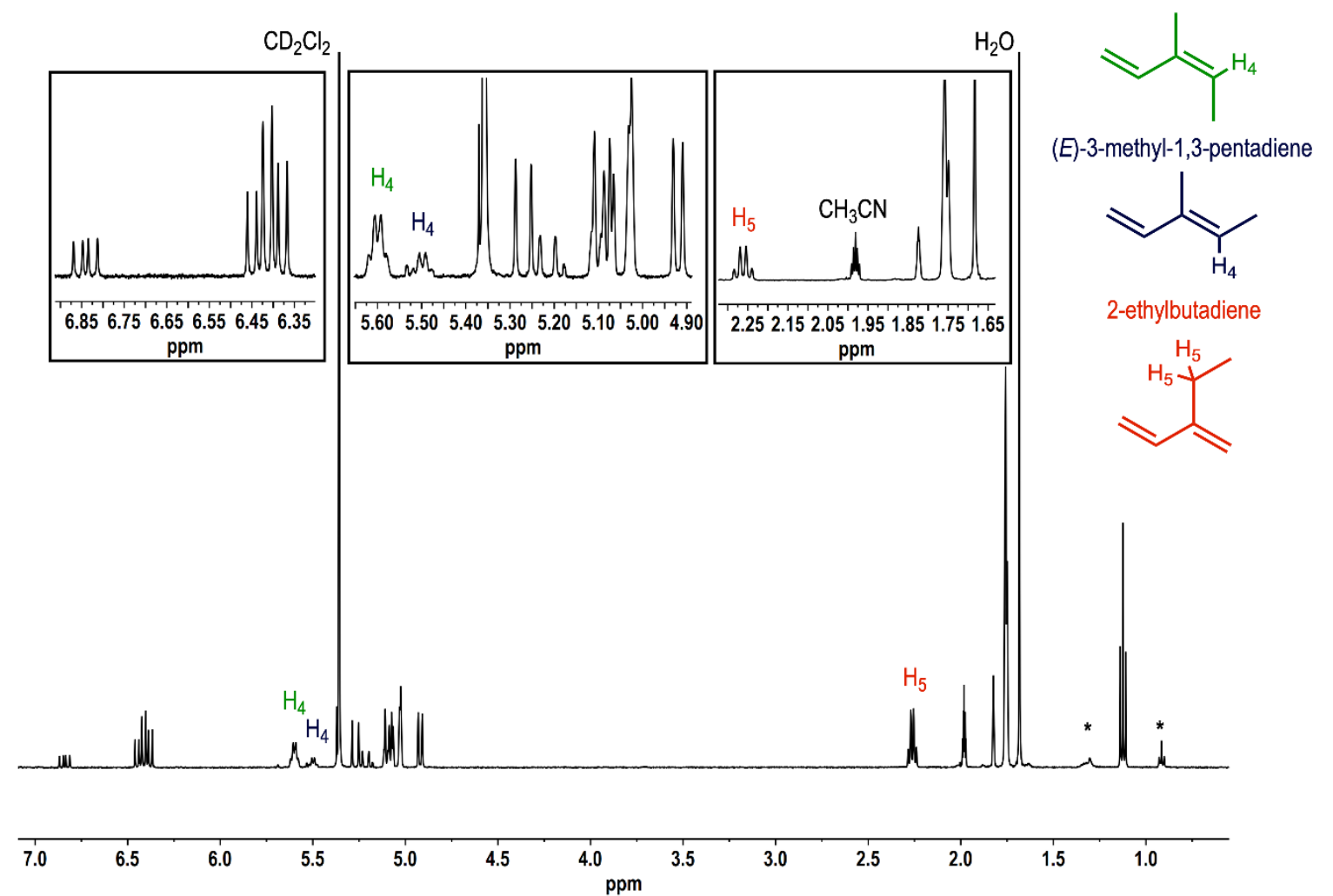

Figure S37: The solution ${ }^{1} \mathrm{H}$ NMR spectrum $\left(\mathrm{CD}_{2} \mathrm{Cl}_{2}, 298 \mathrm{~K}, 500 \mathrm{MHz}\right)$ of isolated $\mathrm{C}_{6} \mathrm{H}_{10}$ volatiles. ${ }^{*}=n$-hexane impurity. The integration of the $\mathrm{H}_{4}: \mathrm{H}_{4}: \mathrm{H}_{5}$ resonances is $0.70: 0.26: 1$. This suggests an $\sim 1$ : 1 ratio of coordinated (Z/E)-3-methyl-1,3-pentadiene and 2-ethylbutadiene. 


\section{S.2.5.4. Synthesis of a 3-methylpentane complex, [1-(3-methylpentane)][BAr $\left.{ }_{4}\right]$}

For Single-Crystal X-Ray Diffraction: Ruby red crystals of [1-( $\left.\left.\mathbf{C}_{6} \mathbf{H}_{10}\right)\right]\left[\mathrm{BAr}^{\mathrm{F}}{ }_{4}\right]\left(\mathrm{C}_{6} \mathrm{H}_{10}=\right.$ 3-methyl-1,3-pentadiene or 2-ethylbutadiene) $(1.5 \mathrm{mgs})$ were placed in a J. Young NMR tube and treated with $\mathrm{H}_{2}$ (1 bar, $298 \mathrm{~K}, 7$ minutes). The colour of the crystals slowly turned a dark red over this time. The crystalline material was then removed from the tube and coated with Fomblin ${ }^{\circledR} Y$ oil under an argon flush. A suitable crystal was then selected and transferred to the cryostream of a diffractometer $(150 \mathrm{~K})$. An X-ray diffraction study was undertaken and the structure of $\left[\mathrm{Rh}\left(\mathrm{Cy}_{2} \mathrm{PCH}_{2} \mathrm{CH}_{2} \mathrm{PCy}\right)\left(\mathrm{C}_{6} \mathrm{H}_{14}\right)\right]\left[\mathrm{BAr}{ }_{4}\right]$ [1-(3-methylpentane)][BAr ${ }_{4}$ ] was refined. For further details of structural refinement please refer to Section S.3.

For Solid-State NMR Spectroscopy: A powdered microcrystalline sample of [1-( $\left.\left.\mathrm{C}_{6} \mathrm{H}_{10}\right)\right]\left[\mathrm{BAr}_{4}{ }_{4}\right]\left(\mathrm{C}_{6} \mathrm{H}_{10}=3\right.$-methyl-1,3-pentadiene or 2-ethylbutadiene) (45 mgs) was packed in a $4.0 \mathrm{~mm}$ SSNMR rotor, inside an argon filled glove box. The rotor was then placed in a custom-built glass $\mathrm{J}$. Young flask ${ }^{\mathrm{S5}}$ and the sample was then exposed to $\mathrm{H}_{2}(1$ bar, $298 \mathrm{~K}$ ). After 20 minutes, the rotor cap was fitted under a flush of $\mathrm{H}_{2}$. The sample was rapidly transferred to the bore of a SSNMR spectrometer ( 10 minutes) and analysed by ${ }^{31} \mathrm{P}\left\{{ }^{1} \mathrm{H}\right\}$ and ${ }^{13} \mathrm{C}\left\{{ }^{1} \mathrm{H}\right\}$ solid-state NMR spectroscopy.

\section{S.2.5.5. Charaterisation data and spectra for [1-(3-methylpentane)][BAr $\left.{ }_{4}\right]$}

${ }^{31} \mathrm{P}\left\{{ }^{1} \mathrm{H}\right\}$ SSNMR (162 MHz, $285 \mathrm{~K}, 10 \mathrm{kHz}$ spin rate): $\delta 107.9\left(\mathrm{~d}, J_{\mathrm{RhP}}=188 \mathrm{~Hz}\right), 105.2(\mathrm{~d}$, $\left.J_{\mathrm{RhP}}=236 \mathrm{~Hz}\right)(\sim 70 \%$ yield $)$.

${ }^{13} \mathrm{C}\left\{{ }^{1} \mathrm{H}\right\}$ SSNMR (101 MHz, $285 \mathrm{~K}, 10 \mathrm{kHz}$ spin rate): $\delta 164.6(\operatorname{ArC}), 135.9(\operatorname{ArC}), 131.5$ (ArC), 130.6 (ArC), 125.5 (ArC), 119.3 (ArC), 118.6 (ArC), 117.1 (ArC), 39.1, 37.8, 36.9, $34.5,33.7,32.2$, 31.4, 30.8, 30.2, 28.2, 26.8, 21.9, 19.2, 11.9, 10.5, 7.63 (multiple aliphatic resonances). 


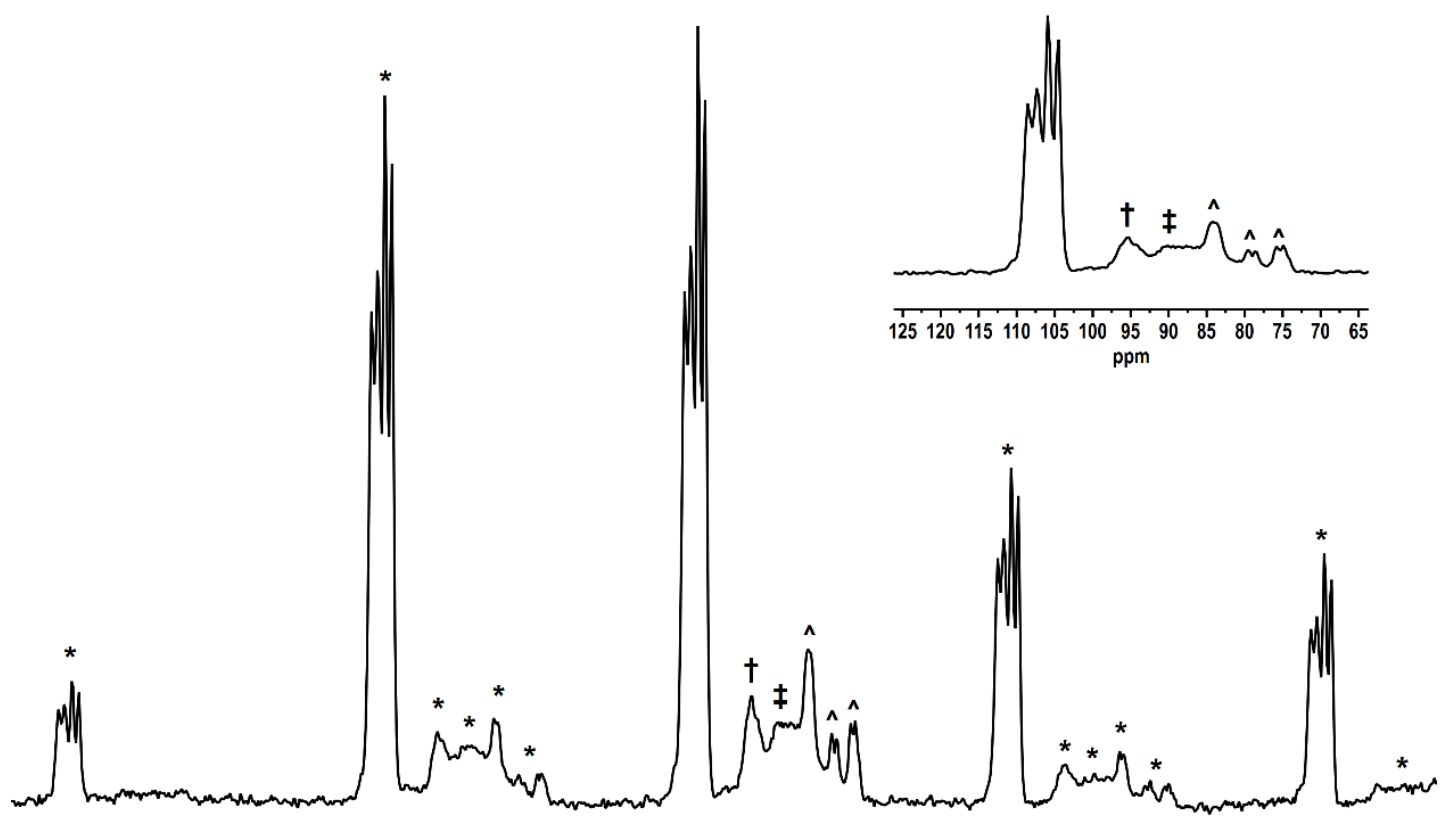

\begin{tabular}{llllllllllllllllllllllllllllllllllll}
\hline 40 & 230 & 220 & 210 & 200 & 190 & 180 & 170 & 160 & 150 & 140 & 130 & 120 & 110 & 100 & 90 & 80 & 70 & 60 & 50 & 40 & 30 & 20 & 10 & 0 & -10 & -20 & -30 & 1
\end{tabular} Figure S38: The ${ }^{31} \mathrm{P}\left\{{ }^{1} \mathrm{H}\right\}$ SSNMR spectrum (162 MHz, $285 \mathrm{~K}, 10 \mathrm{kHz}$ spin rate) of [1-(3-methylpentane)][BAr $\left.{ }_{4}\right]$. Peaks marked * are assigned to spinning sidebands, $\neq$ to $\left[1-\mathrm{BAr}_{4}\right]^{\mathrm{S} 2}$ and ${ }^{\wedge}$ to unreacted [1-( $\left.\left.\mathrm{C}_{6} \mathrm{H}_{10}\right)\right]\left[\mathrm{BAr}_{4}{ }_{4}\right]\left(\mathrm{C}_{6} \mathrm{H}_{10}=3\right.$-methyl-1,3-pentadiene or 2-ethylbutadiene) (see Fig. S36). $\dagger$ is assigned to the dehydrogenation product from [1-(3-methylpentane)][BArF ${ }_{4}$ ]. The inset is an enlargement of the resonances between $\delta 125$ and 65 .

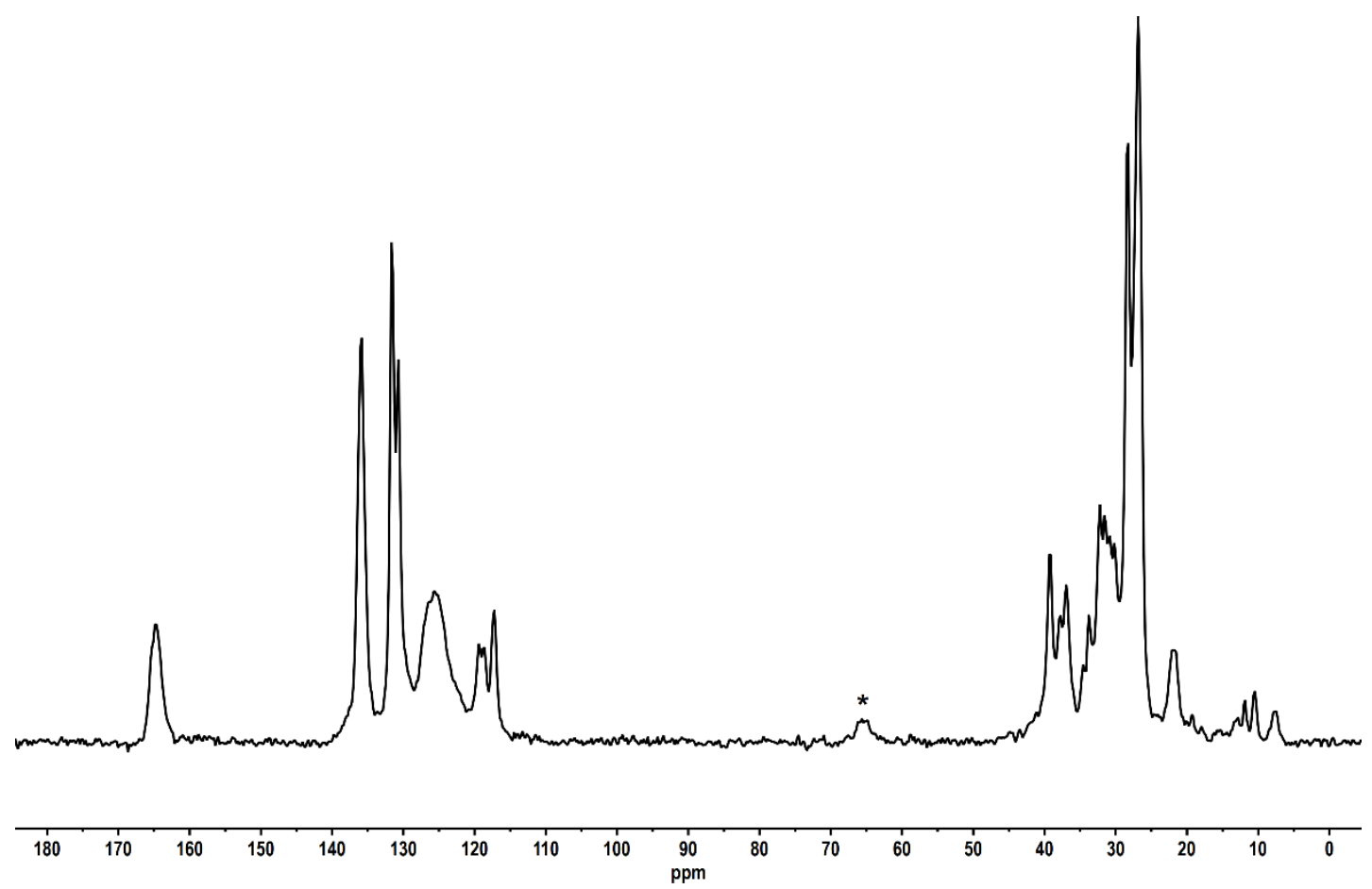

Figure S39: The ${ }^{13} \mathrm{C}\left\{{ }^{1} \mathrm{H}\right\}$ SSNMR spectrum (101 MHz, $285 \mathrm{~K}, 10 \mathrm{kHz}$ spin rate) of [1-(3-methylpentane)][BAr $\left.{ }_{4}\right]$. The resonances marked * are due to spinning sidebands. 


\section{S.2.5.6. Stability of [1-(3-methylpentane)][BAr $\left.{ }_{4}\right]$ at $294 \mathrm{~K}$}

On the same sample as presented in Figure S41 and $\mathrm{S} 42,{ }^{31} \mathrm{P}\left\{{ }^{1} \mathrm{H}\right\}$ SSNMR spectra were collected until the loss of any signals relating to [1-(3-methylpentane)][BAr${ }_{4}$ ] was observed.

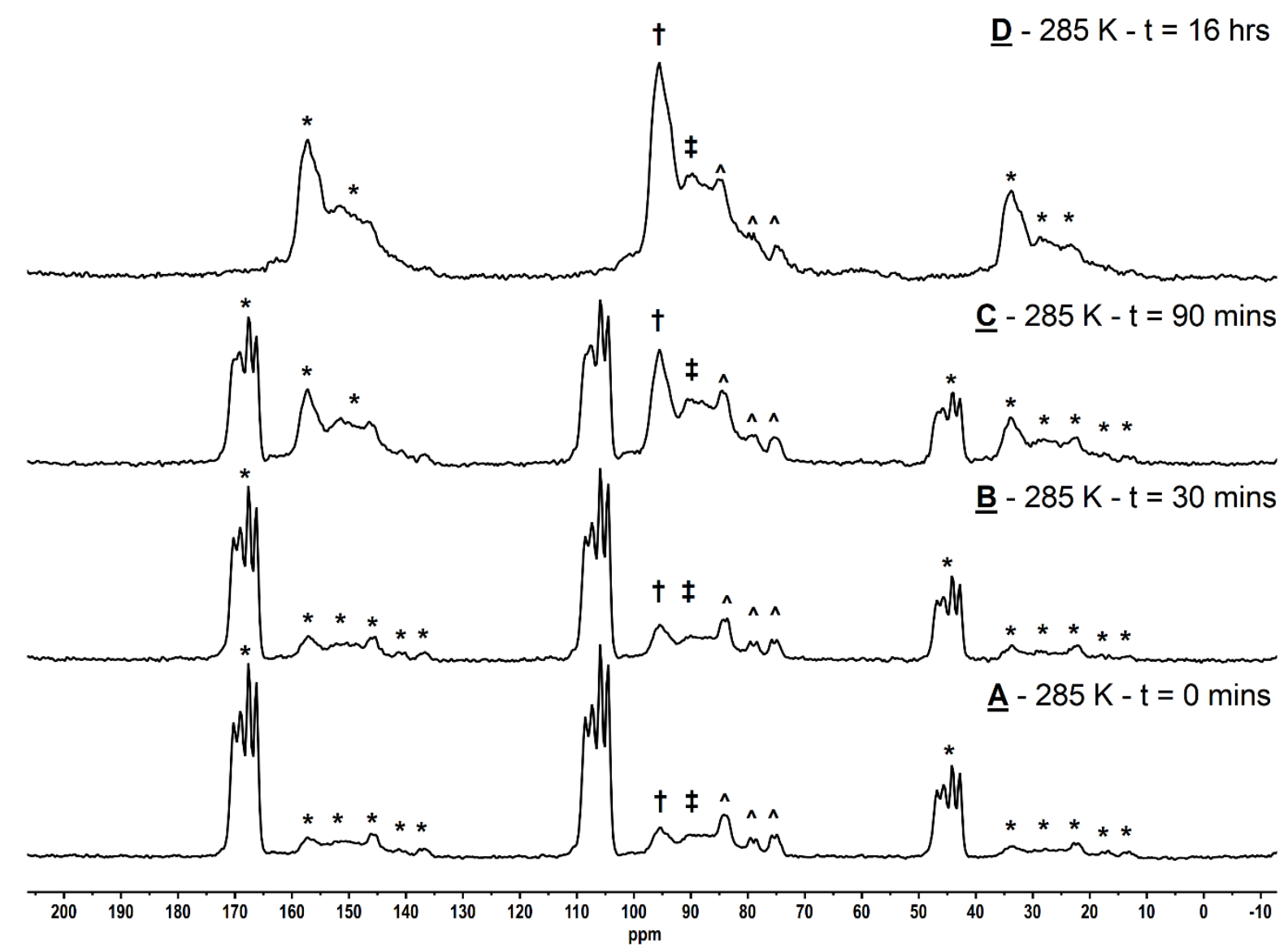

Figure S40: The ${ }^{31} \mathrm{P}\left\{{ }^{1} \mathrm{H}\right\}$ SSNMR spectra (162 MHz, $285 \mathrm{~K}, 10 \mathrm{kHz}$ spin rate) of $\mathbf{A}$ : [1-(3-methylpentane)][BAr ${ }_{4}$ ], as prepared at $285 \mathrm{~K}$. B: Spectrum collected after 20 minutes. C: Spectrum collected after 90 minutes. D: Spectrum collected after 16 hours; where the rotor has been left under a flushing $\mathrm{N}_{2}$ atmosphere during each timepoint. Peaks marked ${ }^{*}$ are assigned to spinning sidebands, $\neq$ to $\left[1-\mathrm{BAr}_{4}\right]^{\mathrm{S2}}$ and ${ }^{\wedge}$ to unreacted [1-( $\left.\left.\mathrm{C}_{6} \mathrm{H}_{10}\right)\right]\left[\mathrm{BAr}_{4}^{\mathrm{F}}\right]\left(\mathrm{C}_{6} \mathrm{H}_{10}=3\right.$-methyl-1,3-pentadiene or 2-ethylbutadiene, see Figure S36). $\dagger$ is assigned to the dehydrogenation product from [1-(3-methylpentane)][BAr ${ }_{4}$ ], (See Section S.2.7.). 


\section{S.2.6. The partial dehydrogenation of [1-(3-methylpentane)][BAr $\left.{ }_{4}\right]$}

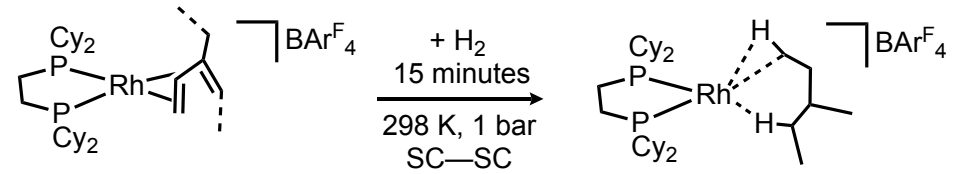

$$
\begin{aligned}
& \left.\left[1-\left(\mathrm{C}_{6} \mathrm{H}_{10}\right)\right]\left[\mathrm{BAr}_{4}{ }_{4}\right] \quad \text { [1-(3-methylpentane)][BArF }{ }_{4}\right]
\end{aligned}
$$

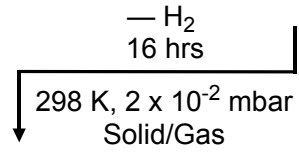

$$
\begin{aligned}
& {\left[\begin{array}{c}
\text { Proposed methylpentene-coordinated complexes }(\sim 65 \%) \\
\text { [1-BArF]( 35\%) }
\end{array}\right.}
\end{aligned}
$$

\section{S.2.6.1. Proposed methylpentenes complex}

As shown in Figure S42D, upon leaving [1-(3-methylpentane)][BAr $\left.{ }_{4}\right]$ under a flushing $\mathrm{N}_{2}$ atmosphere for 16 hours (unoptimised) at ambient temperature, a new signal in the ${ }^{31} \mathrm{P}\left\{{ }^{1} \mathrm{H}\right\}$ SSNMR spectra ( $\delta$ 95.1) is observed growing in. This is assigned to a potential methylpentene-coordinated complex.

The solution ${ }^{31} \mathrm{P}\left\{{ }^{1} \mathrm{H}\right\}$ NMR spectra after this time showed a set of signals at $\left[\delta 93.4, \mathrm{~d}, J_{\mathrm{RhP}}\right.$ $180 \mathrm{~Hz}$, consistent to that of an isobutene ${ }^{\mathrm{S7}}$ and 2-butene ${ }^{\mathrm{S5}}$ coordinated complex, as well as [1-methylbutenes][BAr${ }_{4}{ }_{4}$. The doublet nature at $298 \mathrm{~K}$ suggests a low energy fluxional process, averaging the two ${ }^{31} \mathrm{P}$ environments, as stated for [1-methylbutenes][BAr ${ }_{4}$ ] (Section S.2.4.).

This complex could not be isolated from the [1-BAr${ }_{4}$ ] decomposition product, inhibiting the full characterisation of this dehydrogenation product. The limited significant data gathered upon this complex is shown herein. 


\section{S.2.6.2 Charaterisation data and spectra}

${ }^{1} \mathrm{H}$ NMR $\left(\mathrm{CD}_{2} \mathrm{Cl}_{2}, 298 \mathrm{~K}, 400 \mathrm{MHz}\right.$ ): $\delta 7.72$ (s, 8H, ortho-ArH), 7.66 (s, 4H, para-ArH), 2.191.52 (br m, overlapping aliphatic $\mathrm{CH}$ ), 1.44-0.80 (br m, aliphatic $\mathrm{CH}$ ).

${ }^{31} \mathrm{P}\left\{{ }^{1} \mathrm{H}\right\}$ NMR $\left(\mathrm{CD}_{2} \mathrm{Cl}_{2}, 298 \mathrm{~K}, 162 \mathrm{MHz}\right): \delta 92.9\left(\mathrm{~d}, \mathrm{~J}_{\mathrm{RhP}}=179.9 \mathrm{~Hz}\right)$.

Note: Both [1-methylbutenes][BAr${ }_{4}$ ] (Section S.2.4.) and a previously reported isobutene coordinated complex, ${ }^{\mathrm{S7}}$ show analogues doublets in their ${ }^{31} \mathrm{P}\left\{{ }^{1} \mathrm{H}\right\}$ NMR spectra of $[\delta 94.4, \mathrm{~d}$, $\left.J_{\mathrm{RhP}}=181 \mathrm{~Hz}\right]$ and $\left[\delta 95.3, \mathrm{~d}, J_{\mathrm{RhP}}=179 \mathrm{~Hz}\right]$ respectively.

${ }^{31} \mathrm{P}\left\{{ }^{1} \mathrm{H}\right\}$ SSNMR (162 MHz, $285 \mathrm{~K}, 10 \mathrm{kHz}$ spin rate): $\delta 95.4$ (br, apparent triplet).

Note: Both [1-methylbutenes][BAr${ }_{4}$ ] (Section S.2.4.) and a previously reported isobutene coordinated complex, ${ }^{\mathrm{S7}}$ show analogues resonances in the ${ }^{31} \mathrm{P}\left\{{ }^{1} \mathrm{H}\right\}$ SSNMR spectra at $\delta 94.6$ and 94.8 respectively.

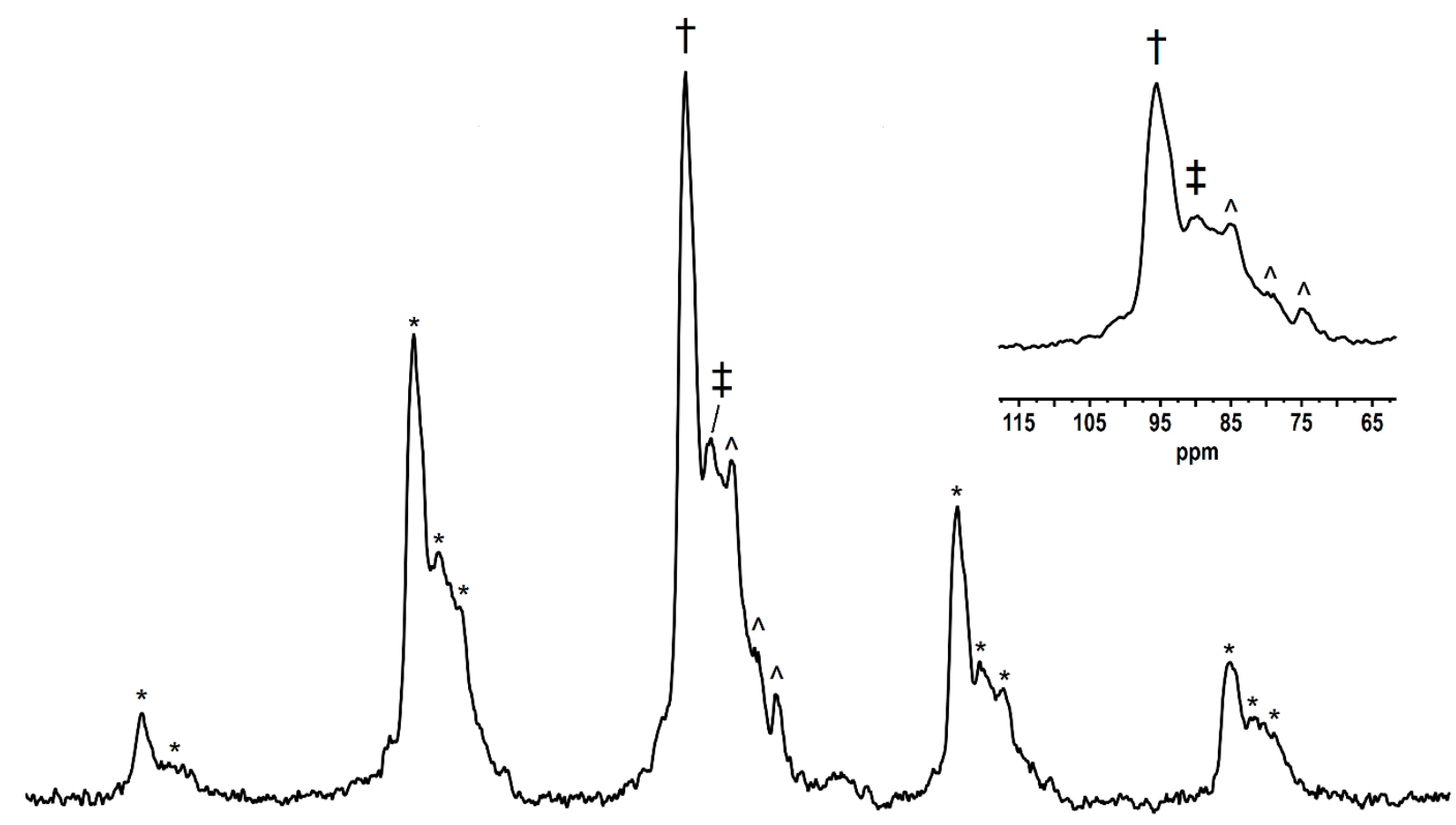

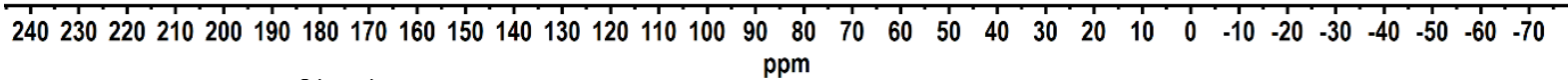
Figure S41: The ${ }^{31} \mathrm{P}\left\{{ }^{1} \mathrm{H}\right\}$ SSNMR spectrum (162 MHz, $285 \mathrm{~K}, 10 \mathrm{kHz}$ spin rate) showing the possible methylpentenes-bound complex, labelled $\dagger$. Peaks marked $\neq$ are assigned to $\left[1-\mathrm{BAr}_{4}\right]^{\mathrm{S} 2}$ and $\wedge$ to $\left[1-\left(\mathrm{C}_{6} \mathrm{H}_{10}\right)\right]\left[\mathrm{BAr}_{4}{ }_{4}\right] \quad\left(\mathrm{C}_{6} \mathrm{H}_{10}=\right.$ 3-methyl-1,3-pentadiene or 2-ethylbutadiene, see Figure S36) from incomplete hydrogenation of the sample. * are assigned to spinning sidebands. 


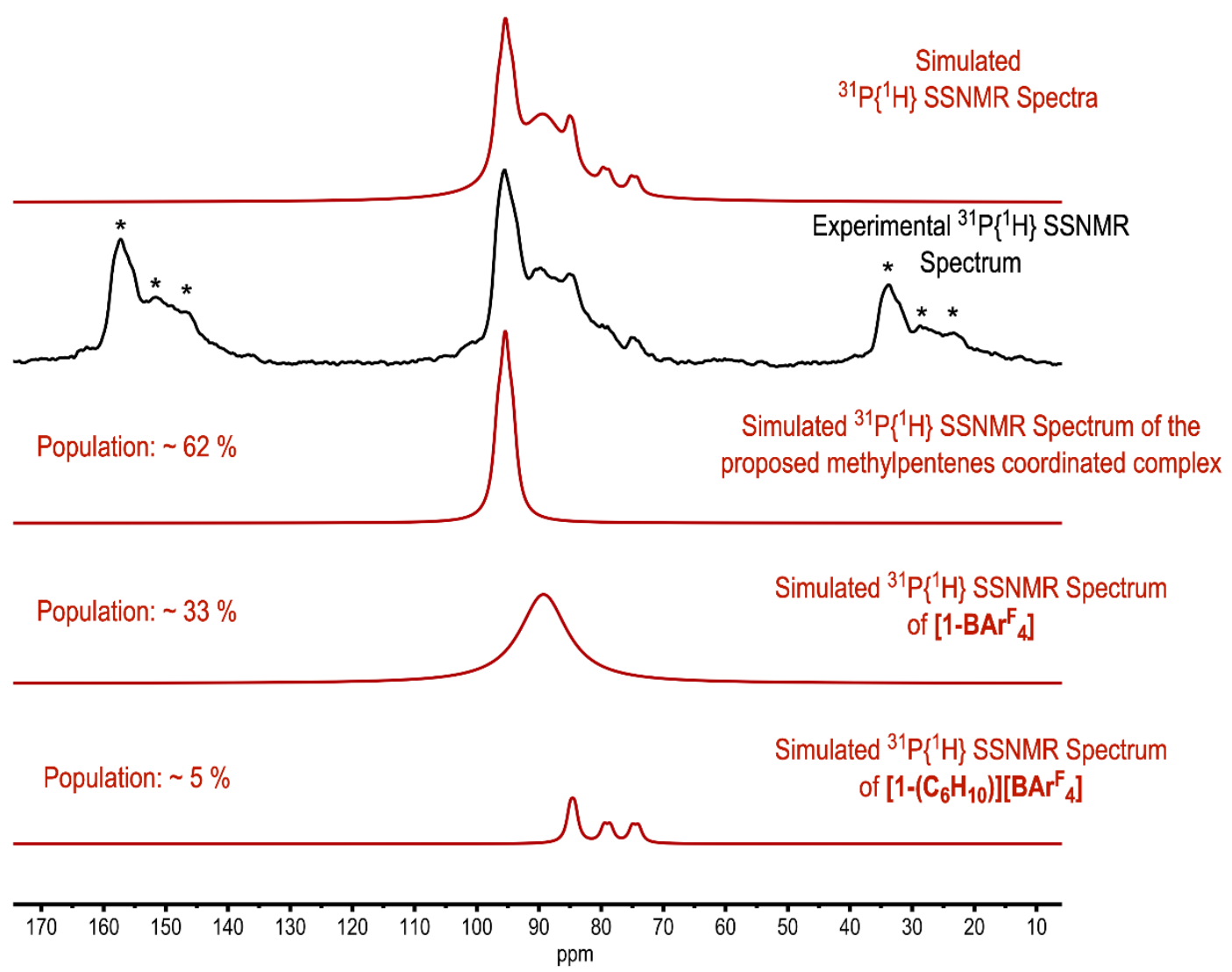

Figure S42: Simulated and experiment ${ }^{31} \mathrm{P}\left\{{ }^{1} \mathrm{H}\right\}$ SSNMR spectra of a potential methylpentene-complex convoluted with known signals from [1-BAr $\left.{ }_{4}\right]$ and [1- $\left.\left(\mathrm{C}_{6} \mathrm{H}_{10}\right)\right]\left[\mathrm{BAr}_{4}{ }_{4}\right]\left(\mathrm{C}_{6} \mathrm{H}_{10}=3\right.$-methyl-1,3-pentadiene or 2-ethylbutadiene). Peaks marked * are assigned to spinning sidebands. The simulated ${ }^{31} \mathrm{P}\left\{{ }^{1} \mathrm{H}\right\}$ SSNMR spectra, suggests an approx. 65: 35 ratios of methylpentene-complex and [1-BAr $\left.{ }_{4}\right]$.

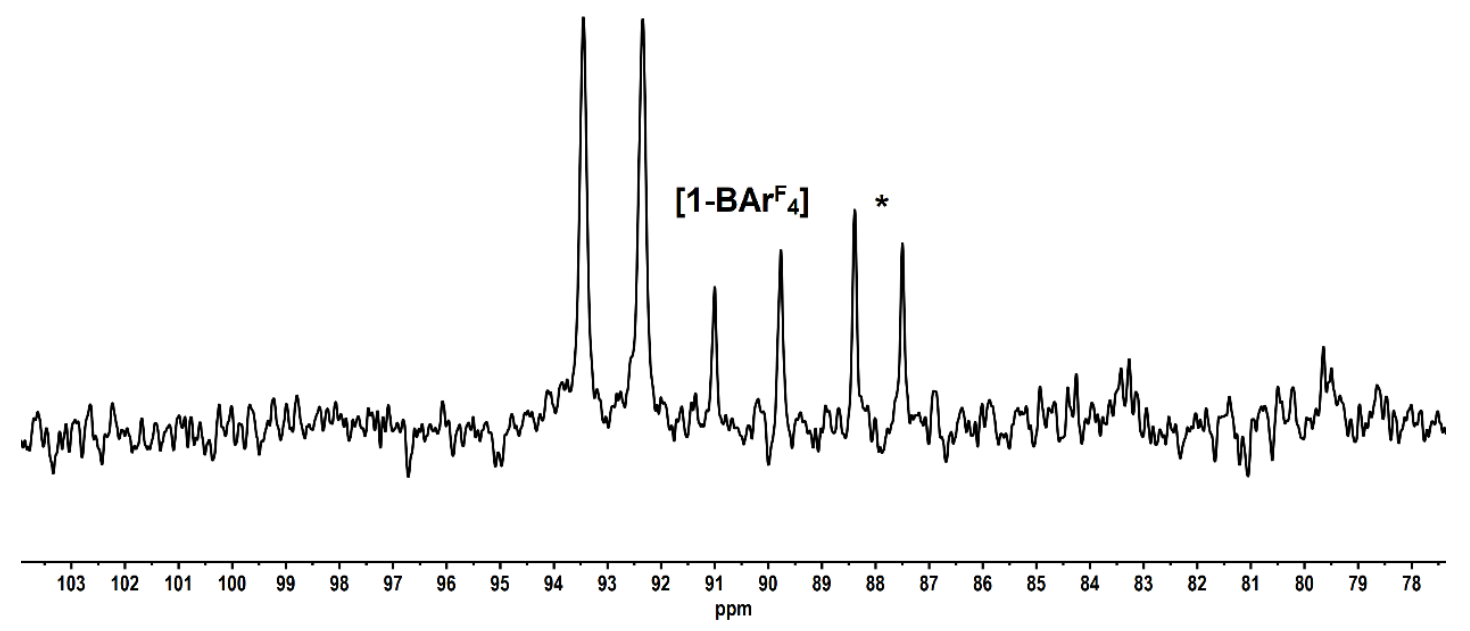

Figure S43: The solution ${ }^{31} \mathrm{P}\left\{{ }^{1} \mathrm{H}\right\} \mathrm{NMR}$ spectrum $\left(\mathrm{CD}_{2} \mathrm{Cl}_{2}, 298 \mathrm{~K}, 162 \mathrm{MHz}\right)$ showing the possible methylpentene-bound complex $(\delta 93),\left[1-\mathrm{BAr}_{4}\right]$ and ${ }^{*}$ is assigned to a further decomposition product of [1-BAr $\left.{ }_{4}\right]$ in $\mathrm{CD}_{2} \mathrm{Cl}_{2}{ }^{\mathrm{S}}{ }^{\mathrm{S}}$ 


\section{S.2.6.3. Liberation of bound methylpentenes}

A J. Young flask was charged with a sample of the proposed methylpentene-complex mixture, as prepared from the same method described in Section S.2.4.3. Dissolution of the solid material in a $\mathrm{C}_{6} \mathrm{D}_{6} /$ Acetone- $d_{6}$ mixture liberated the bound methylpentenes. The resultant organometallic complex was found to be the benzene-coordinated organometallic complex by ${ }^{31} \mathrm{P}\left\{{ }^{1} \mathrm{H}\right\}$ NMR spectroscopy [ठ 99.7, d, $\left.J_{\mathrm{RhP}}=201 \mathrm{~Hz}\right]{ }^{\mathrm{S} 7}$

The reaction volatiles could be isolated by trap-to-trap distillation and the ${ }^{1} \mathrm{H}$ NMR spectrum is shown in Figure S47, with assigned alkene-isomers highlighted. El-MS of these volatiles confirmed the formation of $\mathrm{C}_{6} \mathrm{H}_{12}[\mathrm{~m} / \mathrm{z}$ found 84.0935 (calc. 84.0933)].

Although the exact ratios of methylpentene-isomers in the solid-state was not found, this solution trapping method suggests (E/Z)-3-methyl-2-pentene is the most populous isomer.<smiles>C=C(CC)CC</smiles>

2-ethyl-1-butene<smiles>CCC=C(C)CC</smiles>

(E/Z)-3-methyl-2-pentene

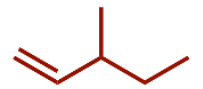

3-methyl-1-pentene
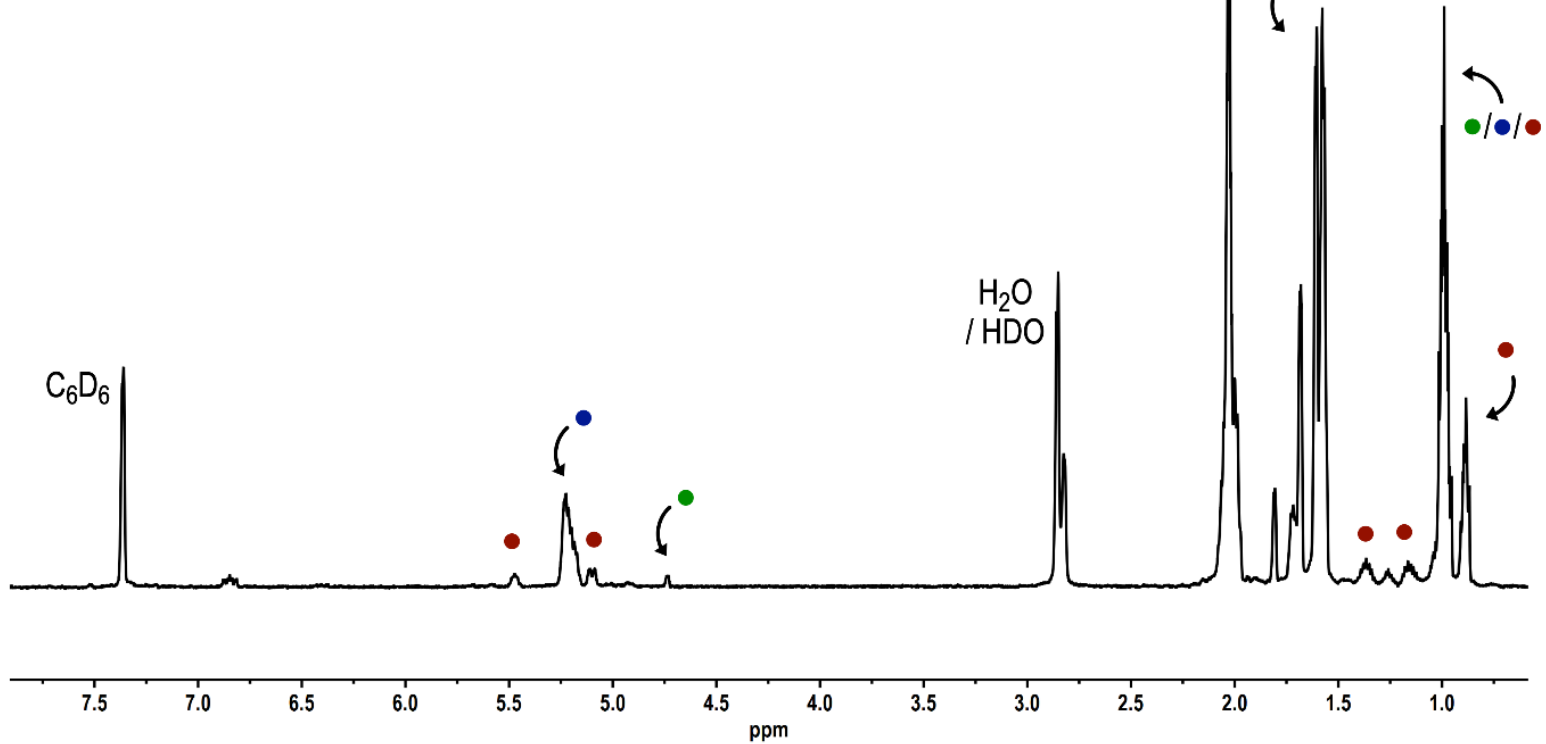

Figure S44: The solution ${ }^{1} \mathrm{H}$ NMR spectrum $\left(\mathrm{CD}_{2} \mathrm{Cl}_{2}, 298 \mathrm{~K}, 500 \mathrm{MHz}\right)$ of isolated alkene volatiles. Signals from the mixture of isomers of 2-ethyl-1-butene, ${ }^{\mathrm{S} 12}$ (E/Z)-3-methyl-2pentene ${ }^{\mathrm{S13}}$ and 3-methyl-1-pentene $\mathrm{S}^{\mathrm{S14}}$ are marked respectively. 

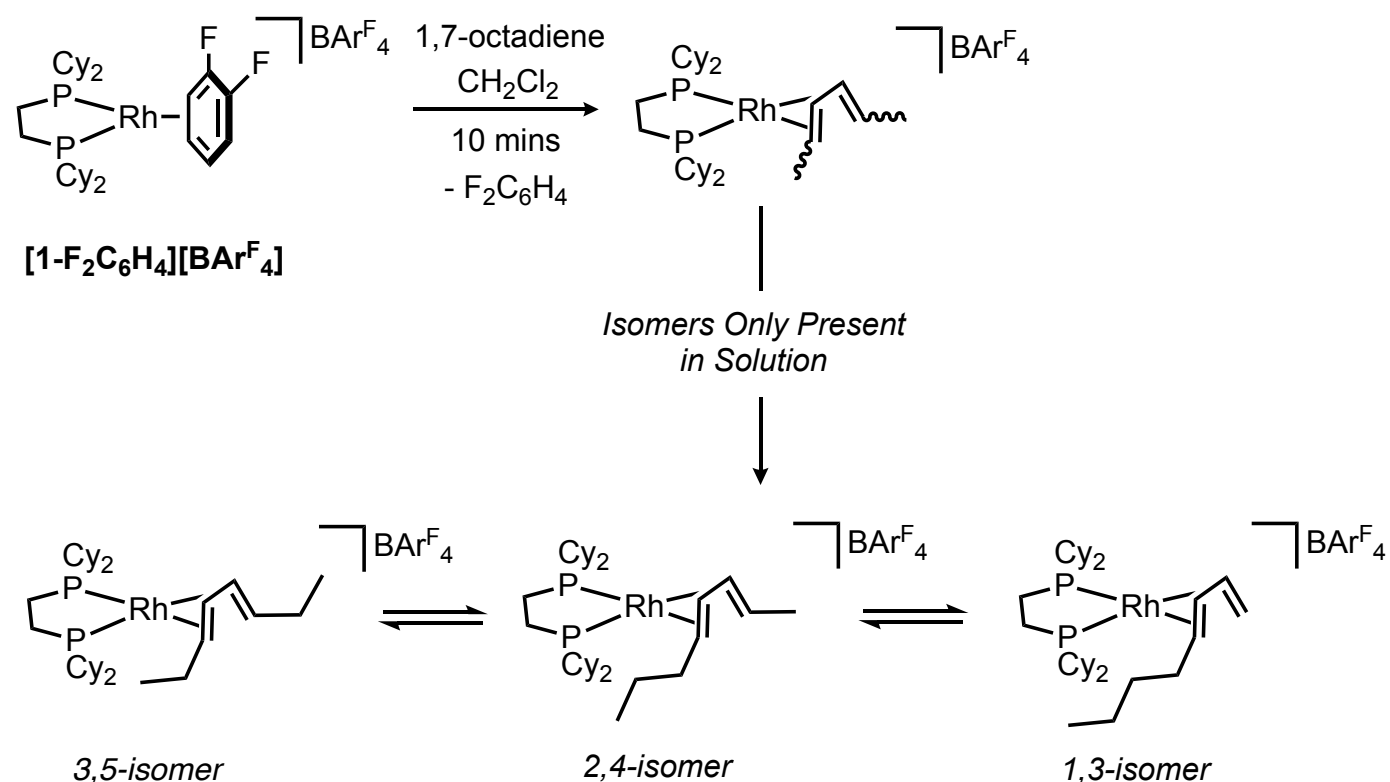
Only Isomer Observed in Solid-State

[1-octadiene][BArF $\left.{ }_{4}\right]$

\section{S.2.7.1. Synthesis of an octodiene complex, [1-octadiene][BAr ${ }_{4}$ ]}

A pale-yellow solution of [1- $\left.\mathbf{F}_{2} \mathbf{C}_{6} \mathrm{H}_{4}\right]\left[\mathrm{BAr}_{4}{ }_{4}\right](600 \mathrm{mgs}, 0.40 \mathrm{mmol})$ in $\mathrm{CH}_{2} \mathrm{Cl}_{2}(20 \mathrm{~mL})$ was treated with neat 1,7-octadiene $(50 \mu \mathrm{L})$ at ambient temperature. The resultant deep red solution was stirred at ambient temperature for 15 minutes. Pentane $(100 \mathrm{~mL})$ was then added with vigorous stirring which resulted in the formation of a red precipitate. The solid was isolated by filtration and washed with pentane $(2 \times 20 \mathrm{~mL})$. The solid was then dissolved in 1,2-dichloroethane $(\sim 12 \mathrm{~mL})$ and filtered into a J. Young crystallisation flask and layered with pentane. Deep red crystals were obtained after storage for 1 week at room temperature of $\left[\mathrm{Rh}\left(\mathrm{Cy}_{2} \mathrm{PCH}_{2} \mathrm{CH}_{2} \mathrm{PCy}_{2}\right)\left(\mathrm{C}_{8} \mathrm{H}_{14}\right)\right]\left[\mathrm{BAr}_{4}{ }_{4}\right]$, [1-octadiene][BAr ${ }_{4}$ ] (Yield: 466 mgs, $78 \%$ ).

Note: The 2,4-isomer was found to be the only isomer present in the solid-state. Taking single-crystals of the 2,4-isomer and re-dissolving in $\mathrm{CH}_{2} \mathrm{Cl}_{2}$ initially showed only 2,4-isomer, before the mixture of isomers growing in within 5 minutes. 


\section{S.2.7.2. Charaterisation data and spectra for [1-octadiene][BAr ${ }_{4}$ ]}

${ }^{1} \mathrm{H}$ NMR $\left(\mathrm{CD}_{2} \mathrm{Cl}_{2}, 298 \mathrm{~K}, 400 \mathrm{MHz}\right.$ ): $\delta 7.72$ (s, 8H, ortho-ArH), 7.57 (s, 4H, para-ArH), 6.65, $6.30,5.56,5.36,5.25,4.16,4.02,2.78$ ( $\mathrm{m}$, multiple alkene resonances from all isomers), 2.47-0.98 (br m, overlapping aliphatic $\mathrm{CH})$.

${ }^{31} \mathrm{P}\left\{{ }^{1} \mathrm{H}\right\}$ NMR $\left(\mathrm{CD}_{2} \mathrm{Cl}_{2}, 298 \mathrm{~K}, 162 \mathrm{MHz}\right): 3,5$-isomer: $\delta 80.7\left(\mathrm{~d}, \mathrm{~J}_{\mathrm{RhP}}=178 \mathrm{~Hz}\right) .2,4$-isomer: $\delta$ $80.9\left(\mathrm{~d}, J_{\mathrm{RhP}}=178 \mathrm{~Hz}, J_{\mathrm{PP}}=24 \mathrm{~Hz}\right), \delta 80.6\left(\mathrm{~d}, J_{\mathrm{RhP}}=178 \mathrm{~Hz}, J_{\mathrm{PP}}=24 \mathrm{~Hz}\right) .1,3$-isomer: $\delta$ $84.5\left(\mathrm{~d}, J_{\mathrm{RhP}}=175 \mathrm{~Hz}, J_{\mathrm{PP}}=22 \mathrm{~Hz}\right), \delta 77.3\left(\mathrm{~d}, J_{\mathrm{RhP}}=173 \mathrm{~Hz}, J_{\mathrm{PP}}=22 \mathrm{~Hz}\right)$.

${ }^{19} \mathrm{~F}\left\{{ }^{1} \mathrm{H}\right\}$ NMR $\left(\mathrm{CD}_{2} \mathrm{Cl}_{2}, 298 \mathrm{~K}, 377 \mathrm{MHz}\right): \delta-62.9(\mathrm{~s})$.

ESI-MS (calc. for $\left.\left[\mathrm{Rh}\left(\mathrm{Cy}_{2} \mathrm{PCH}_{2} \mathrm{CH}_{2} \mathrm{PCy}_{2}\right)\left(\mathrm{C}_{8} \mathrm{H}_{14}\right)\right]^{+}\right): \mathrm{m} / z 635.3329$ (635.3376).

Elemental Analysis (calc. for $\mathrm{C}_{66} \mathrm{H}_{74} \mathrm{BF}_{24} \mathrm{P}_{2} \mathrm{Rh}$ ): $\mathrm{C} 52.73$ (52.88), $\mathrm{H} 4.87$ (4.98).

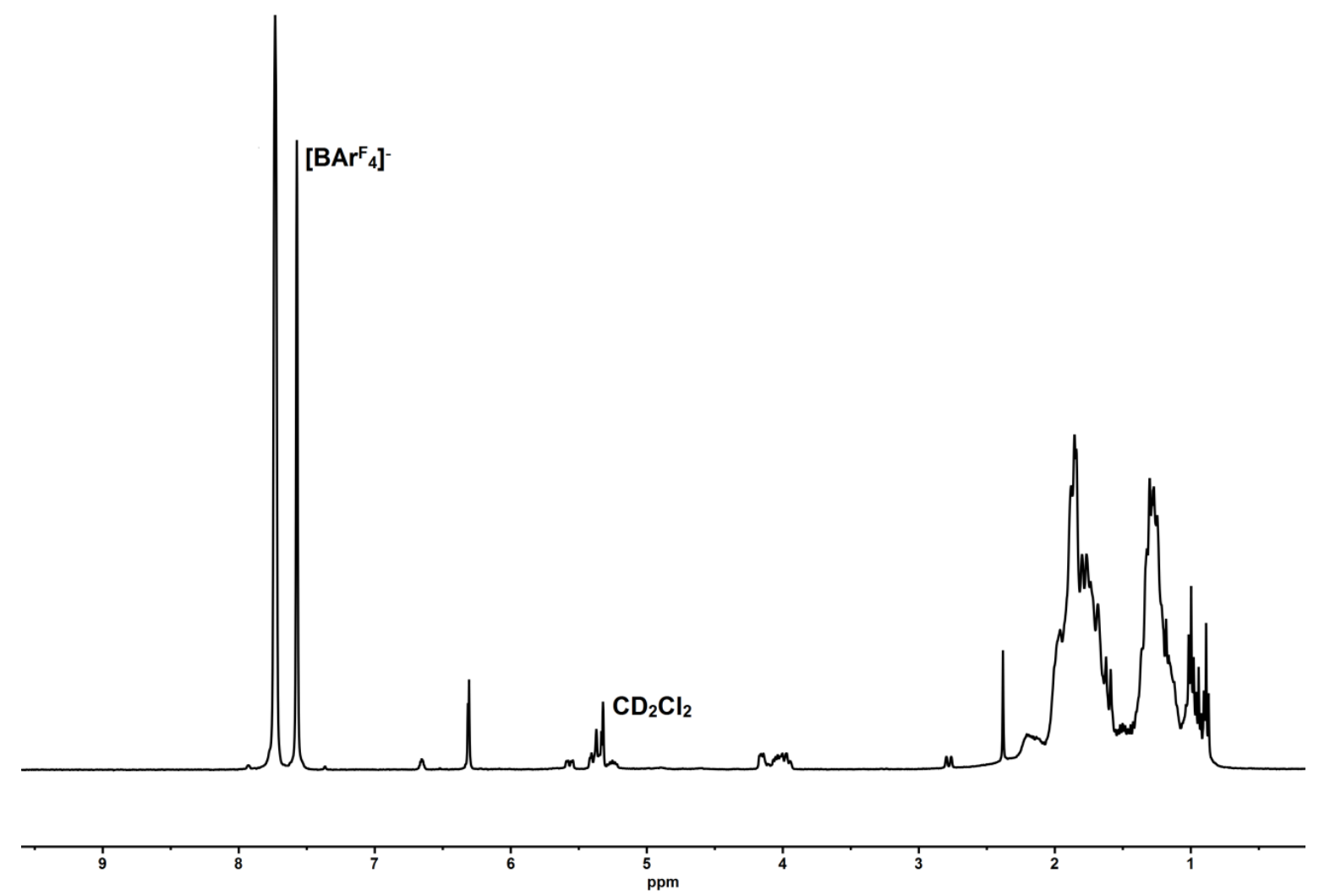

Figure S45: The solution ${ }^{1} \mathrm{H}$ NMR spectrum $\left(\mathrm{CD}_{2} \mathrm{Cl}_{2}, 298 \mathrm{~K}, 400 \mathrm{MHz}\right)$ of [1-octadiene][BArF $\left.{ }_{4}\right]$. 


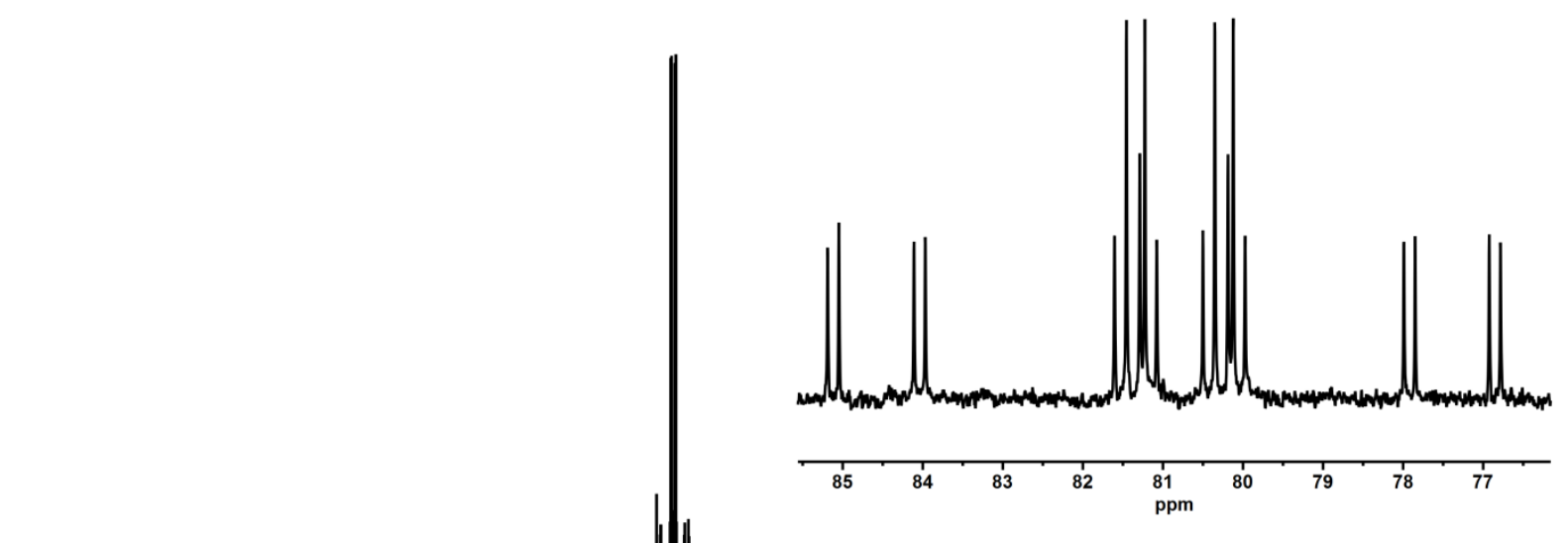

Figure S46: The solution ${ }^{31} \mathrm{P}\left\{{ }^{1} \mathrm{H}\right\} \quad \mathrm{NMR}$ spm spectrum $\left(\mathrm{CD}_{2} \mathrm{Cl}_{2}, 298 \mathrm{~K}, 162 \mathrm{MHz}\right)$ of [1-octadiene][BAr ${ }_{4}$ ]. The inset is an enlargement of the resonances between $\delta 85$ and 77 . 


\section{S.2.7.3. Attempted synthesis of ethane and octane complexes}

Following the previously stated procedures in Sections S.2.3.3 and S.2.4.3., addition of $\mathrm{H}_{2}$ (1 bar, $298 \mathrm{~K}, 10$ minutes) to ruby red crystals the previously reported bis-ethene complex, ${ }^{\mathrm{S} 5}$ $\left[\mathrm{Rh}\left(\mathrm{Cy}_{2} \mathrm{PCH}_{2} \mathrm{CH}_{2} \mathrm{PCy}_{2}\right)\left(\mathrm{C}_{2} \mathrm{H}_{4}\right)_{2}\right]\left[\mathrm{BAr}_{4}\right]$ [1-(ethene $\left.)_{2}\right]\left[\mathrm{BAr}_{4}{ }_{4}\right.$ or [1-octadiene][BAr ${ }_{4}$ ] resulted in the crystals changing colour change to light yellow. It was found in both cases these yellow crystals did not diffract in single-crystal X-ray diffraction experiments.

Dissolution of this product in $\mathrm{CD}_{2} \mathrm{Cl}_{2}$ shows the complete hydrogenation of [1-(ethene) $\left.)_{2}\right]\left[\mathrm{BAr}_{4}{ }_{4}\right]$ and [1-octadiene][BAr $\left.{ }_{4}\right]$, as no signals relating to [1-(ethene) $\left.)_{2}\right]\left[\mathrm{BAr}_{4}{ }_{4}\right]$ or [1-octadiene][BAr ${ }_{4}$ ] were observed in the respective solution ${ }^{31} \mathrm{P}\left\{{ }^{1} \mathrm{H}\right\}$ NMR spectra. Trap-to-trap distillation of the volatiles from these solutions and analysis by ${ }^{1} \mathrm{H}$ NMR spectroscopy confirmed the formation of ethane and octane respectively.

Additionally for [1-(ethene) $)_{2}$ [BAr ${ }_{4}$ ], SSNMR spectroscopy was attempted following the same procedure used in Section S.2.1.2. for [1-propane][BAr ${ }_{4}$ ]. Only signals relating to the decomposition product of [1-BAr ${ }_{4}$ ] were observed $\left({ }^{31} \mathrm{P}\left\{{ }^{1} \mathrm{H}\right\} \mathrm{SSNMR}\right.$ : $\left.\delta 91\right) .{ }^{\mathrm{S} 2}$

For [1-octadiene][BAr ${ }_{4}$ ], any reduction in the time of hydrogenation $(<10$ minutes) resulted in a mix of [1-octadiene][BAr ${ }_{4}$ ] starting material (see Section S.2.8.2.) and [1-BAr ${ }_{4}$ ] $(\delta 91$, d, $\left.J_{\text {RhP }}=175 \mathrm{~Hz}\right)^{S 5}$ product by solution ${ }^{31} \mathrm{P}\left\{{ }^{1} \mathrm{H}\right\}$ NMR spectroscopy. 


\section{S.3 Crystallographic and Refinement Data}

\section{S.3.1. Crystal structure determinations}

Regardless of collection method (described below), all raw frame data were reduced using CrysAlisPro. ${ }^{\mathrm{S} 15}$ The structures were solved using SHELXT ${ }^{\mathrm{S} 16}$ and refined using full-matrix least squares refinement on all $\mathrm{F}^{2}$ data using the SHELXL-18 ${ }^{S 17}$ using the interface OLEX2. ${ }^{\mathrm{S} 18}$ In all structures, disorder of the $-\mathrm{CF}_{3}$ groups was treated by introducing a split site model and restraining geometries and displacement parameters. Distances and angles were calculated using the full covariance matrix.

\section{S.3.1.1. Diamond Light Source}

Gas cell: The $1191 \mathrm{~mm}$ quartz capillary gas cell comprises of a quartz capillary (Hampton research HR6-148, OD $1 \mathrm{~mm}$, ID $0.98 \mathrm{~mm}, \mathrm{~L} 25 \mathrm{~mm}$ ) which is glued into a brass specimen pin (Hampton research HR4-661) using silver epoxy glue and the open end cut to length using a capillary cutting stone (Hampton research HR4-334). The other half of the cell is a $1 / 8$ to $1 / 16$ inch reducing union with $1 / 16$ inch stainless steel capillary tubing (Swagelok SST1-S-014-6ME) to a miniature quick connector (Swagelok SS-QM2-D-100).

Due to the air sensitivity of the systems under investigation the mounting procedure occurs under an inert atmosphere within a glove box. Without the use of manipulation oil, a crystal is selected and mounted, using a minimal amount of epoxy glued, to the tip of a sample mount (mitogen M2-L25SP-30). The mount is then placed into the capillary and fixed in place with a small amount of glue. The two halves of the gas cell assembled creating a gas tight environment for the sample ready to be mounted on the diffractometer and connected to gas rig. 

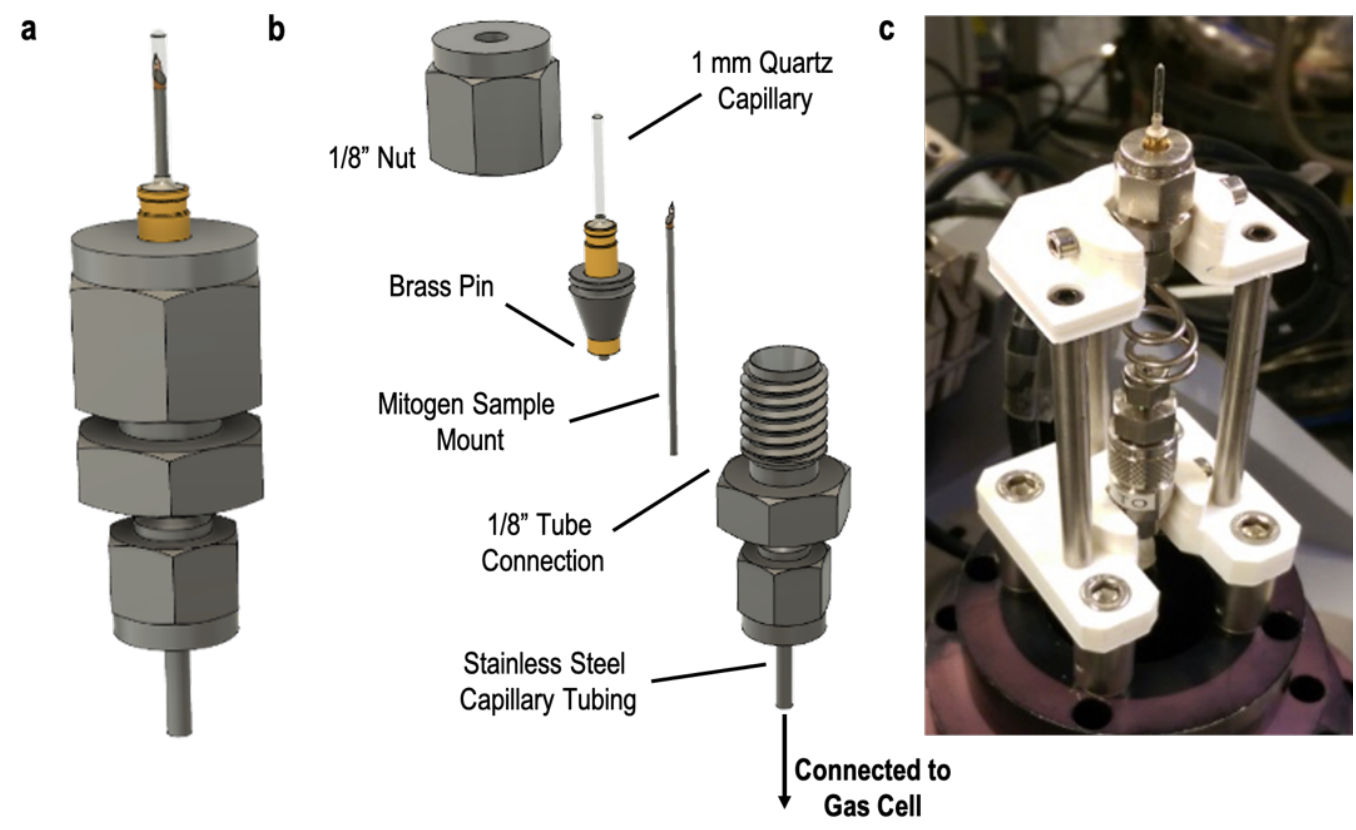

Figure S47: I19 Beamline $1 \mathrm{~mm}$ quartz capillary gas cell equipment. a) Technical drawing of the head of the cell with b) showing the exploded view. c) Image of the diffractometer mounted cell. Image adapted with kind permission from Dr Mark Warren, Diamond Light Source.

Gas Rig: The 119 gas-cell control panel allows the users to operate the gas flow, gas pressure, gas mixture and vacuum remotely. The main rig has several pneumatic valves (V1-6), for connections to samples (S1), sample return (SR1), gases (G1-3), vacuum (Vac1), pressure transducers (PR1-2) and exhaust (EX1) (Figure S51).

The vacuum pump is a scroll pump with a turbo attachment capable of reducing the pressure to $10^{-6} \mathrm{mbar}$ at the pump transducer and $10^{-4} \mathrm{mbar}$ at the sample position. Gas pressure, flows and mixtures can be controlled by the mass flow controllers (Alicat MC-200SCCM-D) and pneumatic valves (V1-3).

The gas-cell on the diffractometer is attached to the control apparatus via 1/16 inch tubing with quick release connectors. The quick release connectors allow users to rapidly and easily attach and remove the cell whilst maintaining the pressure in the cell. The setup of this apparatus allows for the gas-cell to be subjected to vacuum, single gases or gas mixtures either under flow of the gas or with gas pressures up to 200 bar in-situ with the diffraction experiment. The alicat mass flow controllers, pneumatic valves and transducers can be controlled and read through the supporting control software. 

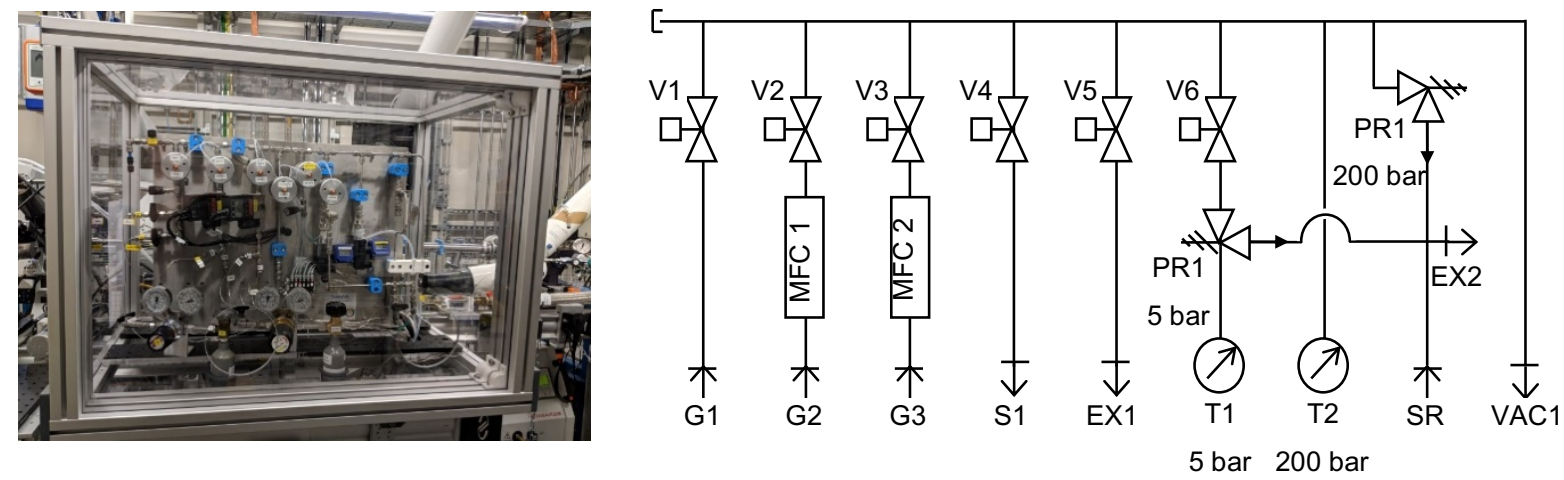

Figure S48: Gas-cell control apparatus schematic. Key: V1-6 pneumatic valves, MFC1-2 alicat mass flow controllers, G1-3 gas inlets, S1 sample connection, EX1-2 exhaust connection, T1-2 pressure transducers, PR1-2 pressure relief valves and VAC1 vacuum connection.

Using the above set-up, the structure of [1-propane][BAr$\left.{ }_{4}\right](150 \mathrm{~K})$ was collected on a Pilatus $300 \mathrm{~K}$ hybrid pixel diffractometer with synchrotron source $\mathrm{Zr}$ K-edge (17.998 keV which equates to $\lambda=0.6889 \AA$ ) on Station I19 at the Diamond Light Source (DLS). ${ }^{\mathrm{S} 6}$ 


\section{S.3.1.2. University of Oxford}

The structures of [1-isoprene][BAr ${ }_{4}$ ], [1-(2-methylbutane)][BAr ${ }_{4}{ }_{4}$, [1-hexadiene][BAr ${ }_{4}$ ], [1-hexane][BAr $\left.{ }_{4}{ }_{4}\right], \quad\left[1-\left(\mathrm{C}_{6} \mathrm{H}_{10}\right)\right]\left[\mathrm{BAr}_{4}{ }_{4}\right], \quad$ [1-(3-methylpentane)][BAr $\left.{ }_{4}\right]$ and [1-octadiene][BAr ${ }_{4}$ ] (all at $150 \mathrm{~K}$ ) were collected at the Oxford Chemical Crystallography Service from the University of Oxford, with an Agilent SuperNova diffractometer $\left(\mathrm{Cu} \mathrm{K}_{\alpha}\right.$ radiation, $\lambda=1.54180 \AA$ ). Single crystal $\mathrm{X}$-ray diffraction data for all samples were collected as follows: a selected crystal was mounted on a MiTeGen Micromounts using perfluoropolyether oil and cooled rapidly to the collection temperature in a stream of nitrogen gas using an Oxford Cryosystems Cryostream unit. ${ }^{\text {s19 }}$

\section{S.3.1.3. National Crystallography Service, Southampton, UK}

[1-methylbutenes][BAr${ }_{4}$ ] $(150 \mathrm{~K})$ was collected at the National Crystallography Service, University of Southampton, Southampton, UK, using a Rigaku 007HF diffractometer equipped with Varimax confocal mirrors $\left(\mathrm{Cu} \mathrm{K}_{\alpha}\right.$ radiation, $\left.\lambda=1.54184 \AA\right)$, an $\mathrm{AFC} 11$ goniometer, and HyPix 6000 detector.

\section{S.3.2. Additional comments on solved structures}

The structures of [1-(2-methylbutane)][BAr ${ }_{4}$ ] and [1-methylbutenes][BAr ${ }_{4}$ ] were refined as a 2-component twins; rotated by $-179.9945^{\circ}$ around $0.710 .71-0.00$ (reciprocal space) or $\begin{array}{llll}0.73 & 0.69 & 0.05 & \text { (direct space) with BASF refined to } 0.4997(13) \text { for }\end{array}$ [1-(2-methylbutane)][BAr ${ }_{4}$ ] and rotated by $-179.9799^{\circ}$ around $0.710 .71-0.00$ (reciprocal space) or 0.720 .690 .03 (direct space) with BASF refined to $0.899(2)$ for [1-methylbutenes][BAr ${ }_{4}$ ]. 


\section{S.3.3. Refinement tables}

Table S1: Selected crystallographic and refinement data.

\begin{tabular}{|c|c|c|c|c|}
\hline & $\begin{array}{l}\text { [1-propane] } \\
{\left[\mathrm{BArF}_{4}\right]}\end{array}$ & $\begin{array}{c}\text { [1-isoprene] } \\
{\left[\mathrm{BArF}_{4}\right]}\end{array}$ & $\begin{array}{c}\text { [1-methylbutenes] } \\
{\left[\mathrm{BArF}_{4}\right]}\end{array}$ & $\begin{array}{c}\text { [1-(2-methylbutane)] } \\
{\left[\mathrm{BArF}_{4}\right]}\end{array}$ \\
\hline Chemical formula & $\mathrm{C}_{61} \mathrm{H}_{68} \mathrm{BF}_{24} \mathrm{P}_{2} \mathrm{Rh}$ & $\mathrm{C}_{63} \mathrm{H}_{68} \mathrm{BF}_{24} \mathrm{P}_{2} \mathrm{Rh}$ & $\mathrm{C}_{63} \mathrm{H}_{70} \mathrm{BF}_{24} \mathrm{P}_{2} \mathrm{Rh}$ & $\mathrm{C}_{63} \mathrm{H}_{72} \mathrm{BF}_{24} \mathrm{P}_{2} \mathrm{Rh}$ \\
\hline Formula weight & 1432.81 & 1456.83 & 1458.85 & 1460.86 \\
\hline Temperature (K) & 150 & 150 & 100 & 150 \\
\hline Crystal system & triclinic & triclinic & triclinic & triclinic \\
\hline Space group & $P-1$ & $P-1$ & $P-1$ & $P-1$ \\
\hline$a(\AA)$ & $12.9862(8)$ & $12.7425(3)$ & $12.7343(2)$ & $12.7559(6)$ \\
\hline$b(\AA)$ & $13.0857(8)$ & $12.9552(3)$ & $13.0256(2)$ & $13.1978(5)$ \\
\hline$c(\AA)$ & $19.9409(11)$ & $20.0752(4)$ & $19.8332(4)$ & $19.7970(5)$ \\
\hline$\alpha(\mathrm{deg})$ & $91.206(5)$ & $92.435(2)$ & $92.6533(16)$ & $93.734(2)$ \\
\hline$\beta$ (deg) & $90.367(5)$ & $91.227(2)$ & $91.1282(16)$ & $92.418(3)$ \\
\hline$V(\mathrm{deg})$ & $96.291(5)$ & $97.363(2)$ & $96.6525(15)$ & $95.556(3)$ \\
\hline$V\left(\AA^{3}\right)$ & $3367.3(4)$ & $3282.54(13)$ & $3263.06(11)$ & $3306.2(2)$ \\
\hline$Z$ & 2 & 2 & 2 & 2 \\
\hline$\rho$ (calcd) $\left(\mathrm{g} \mathrm{cm}^{-3}\right)$ & 1.413 & 1.474 & 1.485 & 1.467 \\
\hline$\mu\left(\mathrm{mm}^{-1}\right)$ & 0.374 & 3.519 & 3.540 & 3.494 \\
\hline Reflections collected & 16866 & 51527 & 13689 & 26345 \\
\hline Unique reflections & 6569 & 13739 & 13689 & 26345 \\
\hline Restraints / Parameters & $1212 / 1028$ & $399 / 999$ & $508 / 956$ & $571 / 975$ \\
\hline$R_{\text {int }}$ & 0.0532 & 0.0512 & & \\
\hline$R_{1}[I>2 \sigma(I)]$ & 0.1073 & 0.0575 & 0.0861 & 0.0930 \\
\hline$w R_{2}$ [all data] & 0.2812 & 0.1679 & 0.2666 & 0.2622 \\
\hline GooF & 1.100 & 1.019 & 1.145 & 0.997 \\
\hline $\begin{array}{l}\text { Residual electron } \\
\text { density }\left(e \AA^{-3}\right)\end{array}$ & $2.25 /-0.78$ & $1.32 /-0.71$ & $0.93 /-1.74$ & $3.40 /-1.02$ \\
\hline CCDC no. & 2056862 & 2056864 & 2056859 & 2056863 \\
\hline
\end{tabular}




\begin{tabular}{|c|c|c|c|c|}
\hline & $\begin{array}{c}\text { [1-hexadiene] } \\
{\left[\mathrm{BArF}_{4}\right]}\end{array}$ & $\begin{array}{c}\text { [1-hexane] } \\
\left.\text { [BArF }{ }_{4}\right]\end{array}$ & $\begin{array}{c}{\left[1-\left(\mathrm{C}_{6} \mathrm{H}_{10}\right)\right]} \\
{\left[\mathrm{BAr}_{4}\right]^{a}}\end{array}$ & $\begin{array}{c}\text { [1-(3-methylpentane) }] \\
{\left[\mathrm{BArF}_{4}\right]}\end{array}$ \\
\hline Chemical formula & $\mathrm{C}_{64} \mathrm{H}_{70} \mathrm{BF}_{24} \mathrm{P}_{2} \mathrm{Rh}$ & $\mathrm{C}_{64} \mathrm{H}_{74} \mathrm{BF}_{24} \mathrm{P}_{2} \mathrm{Rh}$ & $\mathrm{C}_{64} \mathrm{H}_{70} \mathrm{BF}_{24} \mathrm{P}_{2} \mathrm{Rh}$ & $\mathrm{C}_{64} \mathrm{H}_{74} \mathrm{BF}_{24} \mathrm{P}_{2} \mathrm{Rh}$ \\
\hline Formula weight & 1470.86 & 1474.89 & 1470.86 & 1474.89 \\
\hline Temperature $(\mathrm{K})$ & 150 & 150 & 150 & 150 \\
\hline Crystal system & triclinic & triclinic & triclinic & triclinic \\
\hline Space group & $P-1$ & $P-1$ & $P-1$ & $P-1$ \\
\hline$a(\AA)$ & $12.8807(3)$ & $13.1078(2)$ & $12.7317(7)$ & $12.6885(2)$ \\
\hline$b(\AA)$ & $13.3277(4)$ & $13.6868(3)$ & $13.1412(6)$ & $13.2056(2)$ \\
\hline$c(\AA)$ & $20.1295(6)$ & $19.1309(3)$ & $20.1179(7)$ & $20.0728(3)$ \\
\hline$\alpha(\mathrm{deg})$ & $101.803(2)$ & $99.0834(15)$ & $94.535(5)$ & $94.5410(10)$ \\
\hline$\beta$ (deg) & $97.376(2)$ & $95.1697(14)$ & $92.043(4)$ & $91.6930(10)$ \\
\hline$V(\mathrm{deg})$ & $100.681(2)$ & $99.1623(15)$ & $96.288(4)$ & $95.6740(10)$ \\
\hline$V\left(\AA^{3}\right)$ & $3273.92(16)$ & $3322.42(10)$ & $3331.9(3)$ & $3334.03(9)$ \\
\hline$z$ & 2 & 2 & 2 & 2 \\
\hline$\rho$ (calcd) $\left(\mathrm{g} \mathrm{cm}^{-3}\right)$ & 1.492 & 1.474 & 1.466 & 1.469 \\
\hline$\mu\left(\mathrm{mm}^{-1}\right)$ & 3.543 & 3.483 & 3.473 & 3.471 \\
\hline Reflections collected & 35413 & 36508 & 28618 & 62097 \\
\hline Unique reflections & 13553 & 13707 & 13907 & 13909 \\
\hline $\begin{array}{l}\text { Restraints / } \\
\text { Parameters }\end{array}$ & $571 / 1010$ & $597 / 1003$ & $1491 / 1112$ & $710 / 983$ \\
\hline$R_{\text {int }}$ & 0.0356 & 0.0320 & 0.0376 & 0.0337 \\
\hline$R_{1}[I>2 \sigma(I)]$ & 0.0402 & 0.0408 & 0.0726 & 0.0483 \\
\hline$w R_{2}$ [all data] & 0.1064 & 0.1108 & 0.2144 & 0.1295 \\
\hline GooF & 1.030 & 1.021 & 1.047 & 1.048 \\
\hline $\begin{array}{l}\text { Residual electron } \\
\text { density }\left(\mathrm{e} \AA^{-3}\right)\end{array}$ & $1.42 /-0.95$ & $0.92 /-0.91$ & $1.49 /-1.33$ & $1.29 /-1.05$ \\
\hline CCDC no. & 2056866 & 2056857 & 2056860 & 2056858 \\
\hline
\end{tabular}

$a=\mathrm{C}_{6} \mathrm{H}_{10}=3$-methyl-1,3-pentadiene or 2-ethylbutadiene. 


\begin{tabular}{|c|c|}
\hline & $\begin{array}{l}\text { [1-octadiene] } \\
{\left[\mathrm{BArF}_{4}\right]}\end{array}$ \\
\hline Chemical formula & $\mathrm{C}_{66} \mathrm{H}_{74} \mathrm{BF}_{24} \mathrm{P}_{2} \mathrm{Rh}$ \\
\hline Formula weight & 1498.91 \\
\hline Temperature (K) & 150 \\
\hline Crystal system & monoclinic \\
\hline Space group & $\mathrm{P} 2{ }_{1} / \mathrm{c}$ \\
\hline$a(\AA)$ & $22.8949(2)$ \\
\hline$b(\AA)$ & $13.1174(1)$ \\
\hline$c(\AA)$ & $25.4290(10)$ \\
\hline$\alpha(\mathrm{deg})$ & 90 \\
\hline$\beta$ (deg) & $114.565(1)$ \\
\hline$V(\operatorname{deg})$ & 90 \\
\hline$V\left(\AA^{3}\right)$ & $6945.67(13)$ \\
\hline$Z$ & 4 \\
\hline$\rho$ (calcd) $\left(\mathrm{g} \mathrm{cm}^{-3}\right)$ & 1.433 \\
\hline$\mu\left(\mathrm{mm}^{-1}\right)$ & 3.342 \\
\hline Reflections collected & 84898 \\
\hline Unique reflections & 14458 \\
\hline Restraints / Parameters & $601 / 1016$ \\
\hline$R_{\text {int }}$ & 0.0357 \\
\hline$R_{1}[I>2 \sigma(I)]$ & 0.0477 \\
\hline$w R_{2}$ [all data] & 0.1387 \\
\hline GooF & 1.018 \\
\hline $\begin{array}{l}\text { Residual electron density } \\
\qquad\left(\mathrm{e} \AA^{-3}\right)\end{array}$ & $1.38 /-0.70$ \\
\hline CCDC no. & 2056865 \\
\hline
\end{tabular}




\section{S.3.4. Structural images from single crystal X-ray diffraction experiments}

A

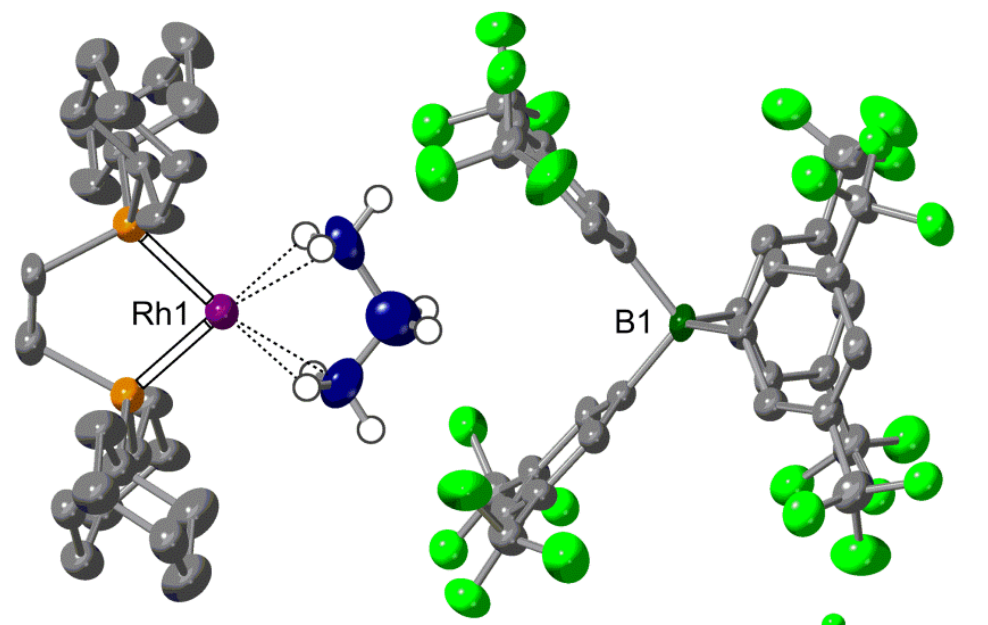

B
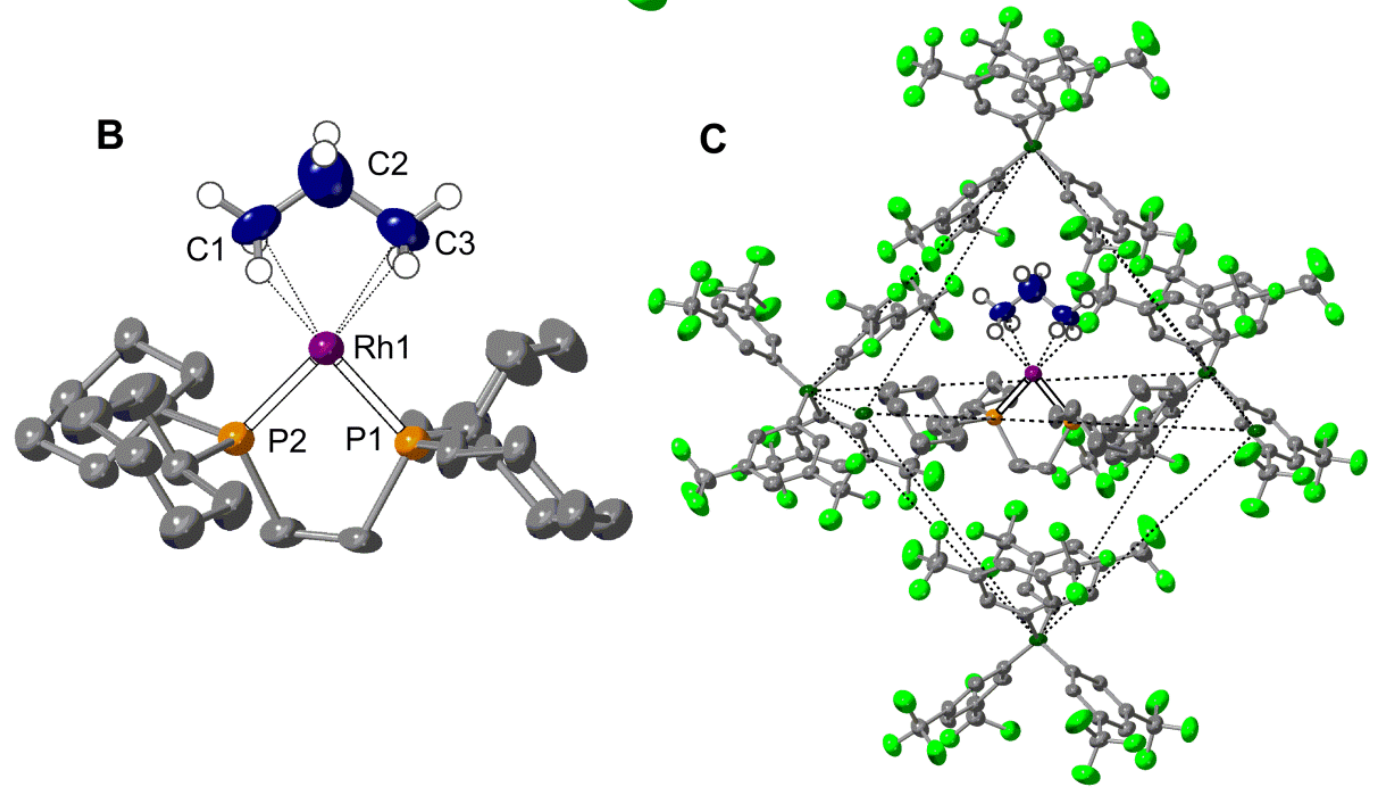

Figure S49: (A) Molecular structure of [1-propane][BArF ${ }_{4}$ ]. (B) Cationic fragment of [1-propane][BAr$\left.{ }_{4}\right]$ showing atom labelling. (C) Structure displaying pseudo- $O_{\mathrm{h}}$ anion network of the $\left[\mathrm{BAr}_{4}\right]^{-}$ion, with cationic fragment sat within the cavity; - $\left(\mathrm{Ar}_{4}{ }_{4}\right)$ removed from 2 boron atoms for clarity. Displacement ellipsoids set at $50 \%$ and hydrogen from phosphine ligand and $\left[\mathrm{BAr}_{4}\right]^{-}$anion removed for clarity in all parts.

Selected bond lengths (Å): Rh1-P1 2.206(4), Rh1-P2 2.226(6), Rh1-C1 2.459(19), Rh1-C3 2.453(19), C1-C2 1.54(2), C2-C3 1.52(2). 
A
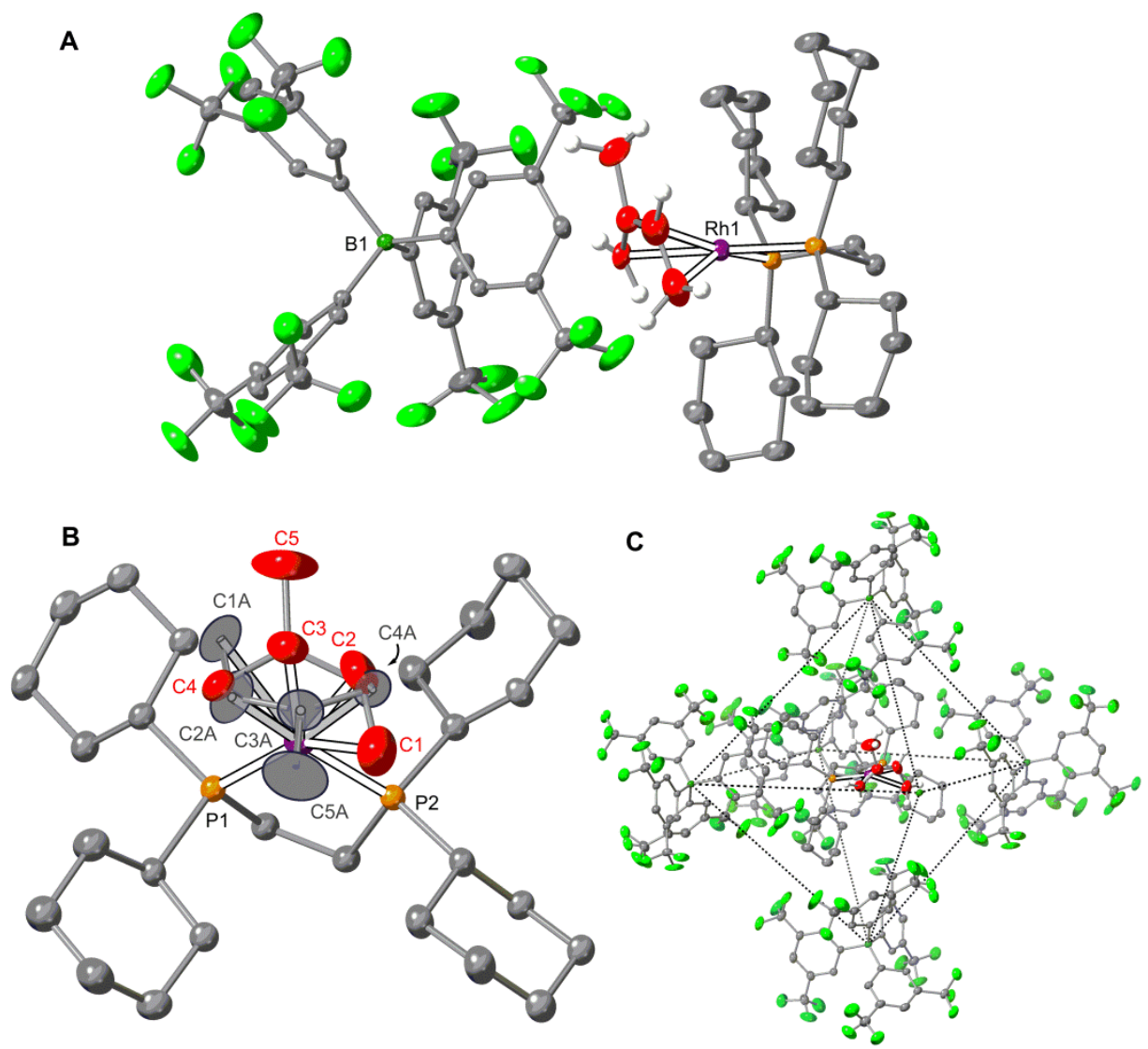

D

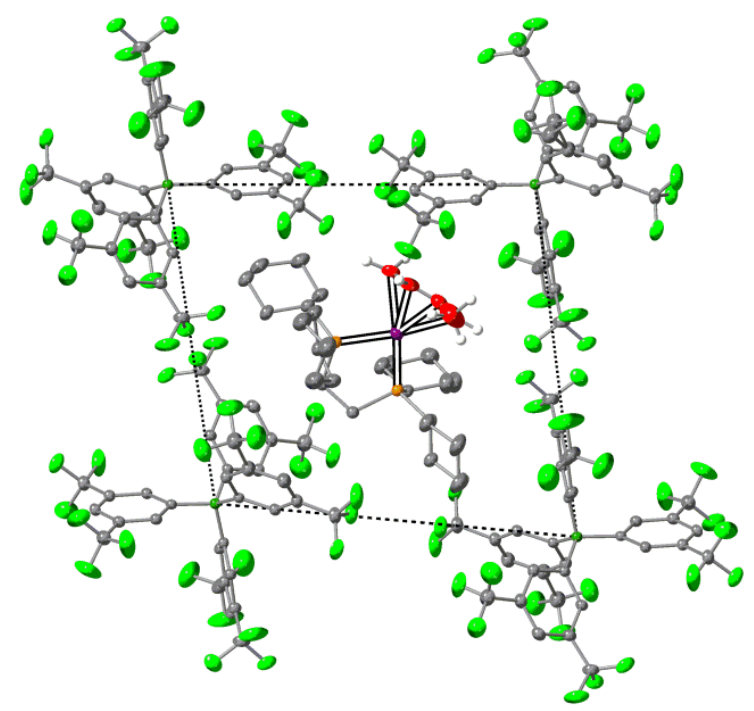

Figure S50: (A) Molecular structure of [1-isoprene][BAr $\left.{ }_{4}\right]$. (B) Cationic fragment of [1-isoprene][BAr$\left.{ }_{4}\right]$ showing major disorder components, where the ratio of non-crystallographically imposed disorder components is $0.51: 0.49$ for major: minor. (C) Structure displaying pseudo- $\mathrm{O}_{\mathrm{h}}$ anion network of the $\left[\mathrm{BAr}_{4}\right]^{-}$ions, with cationic fragments sat within the cavity; $-\left(\mathrm{ArF}_{4}\right)$ removed from 1 boron atom for clarity. (D) Top view of pseudo- $O_{n}$ anion-cage, showing linear cation/anion orientation. Displacement ellipsoids set at $50 \%$ and hydrogen from phosphine ligand and [BArF $\left.{ }_{4}\right]^{-}$ anion removed for clarity in all parts.

Selected bond lengths (A): Rh1-P1 2.2815(9), Rh1-P2 2.2708(9), Rh1-C1 2.279(16), Rh1-C2 2.167(8), Rh1-C3 2.117(19), Rh1-C4 2.313(9), C1-C2 1.34(2), C3-C4 1.31(2). 

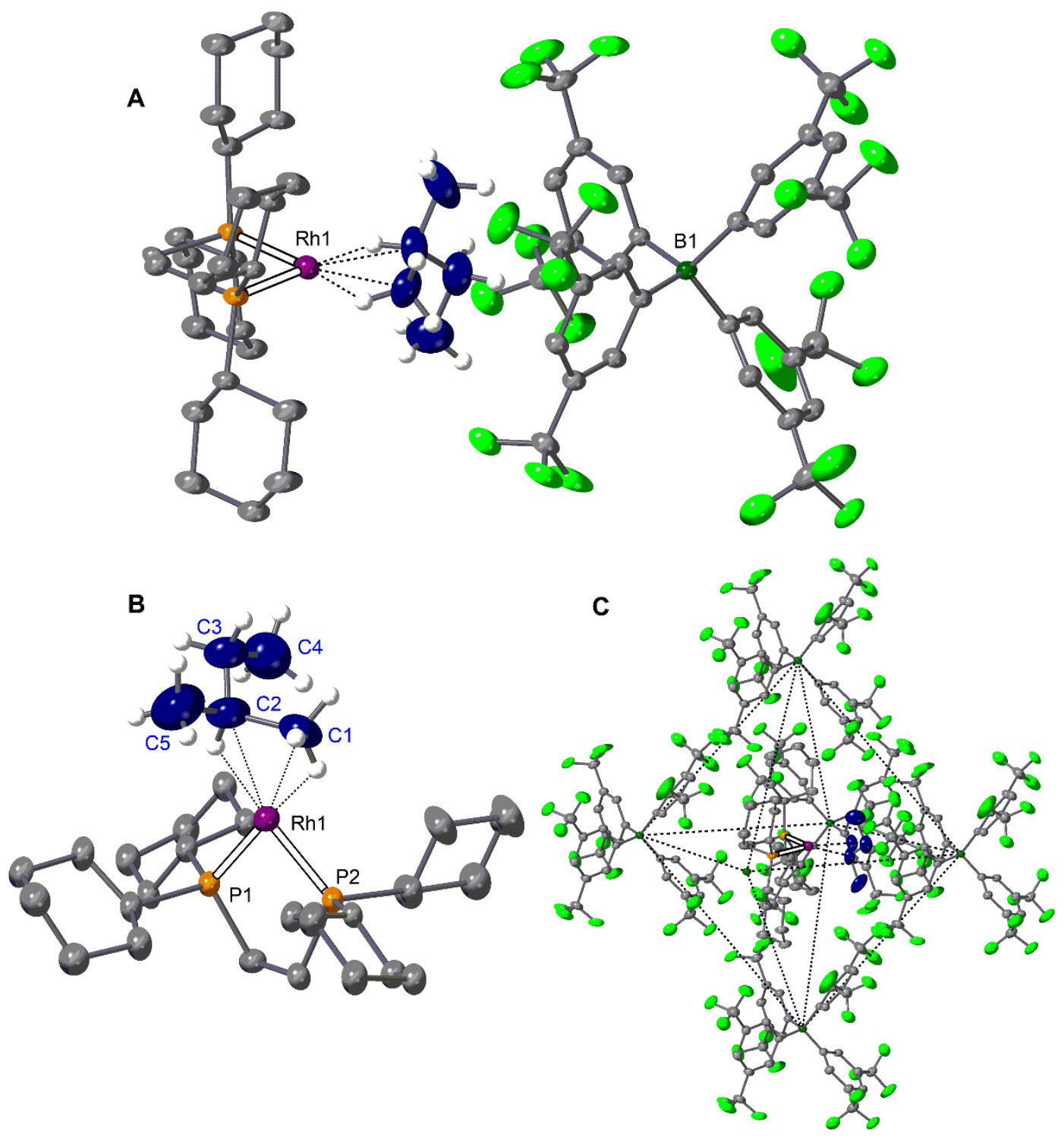

Figure S51: (A) Molecular structure of [1-(2-methylbutane)][BAr $\left.{ }_{4}\right]$. (B) Cationic fragment of [1-(2-methylbutane)][BAr${ }_{4}$ ] showing expanded cation view and atom labelling. (C) Structure displaying pseudo- $\mathrm{O}_{\mathrm{h}}$ network of the $\left[\mathrm{BAr}_{4}\right]^{-}$ions, with cationic fragments sat within the cavity; $-\left(\mathrm{Ar}_{4}{ }_{4}\right)$ removed from 4 boron atoms for clarity. Displacement ellipsoids set at $50 \%$ and hydrogen from phosphine ligand and $\left[\mathrm{BAr}_{4}\right]^{-}$anion removed for clarity in all parts.

Selected bond lengths $(\AA ̊)$ : Rh1-P1 2.1869(16), Rh1-P2 2.1848(15), Rh1-C1 2.348(9), Rh1C2 2.391(10), C1-C2 1.599(13), C2-C3 1.456(14), C2-C5 1.551(15), C3-C4 1.512(16). 
A

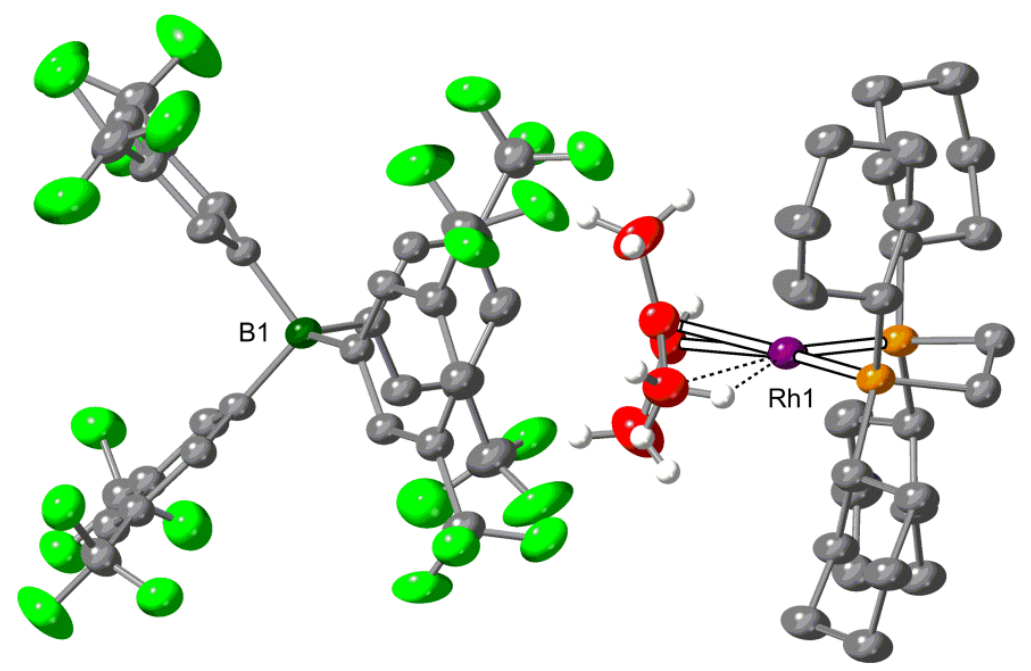

B

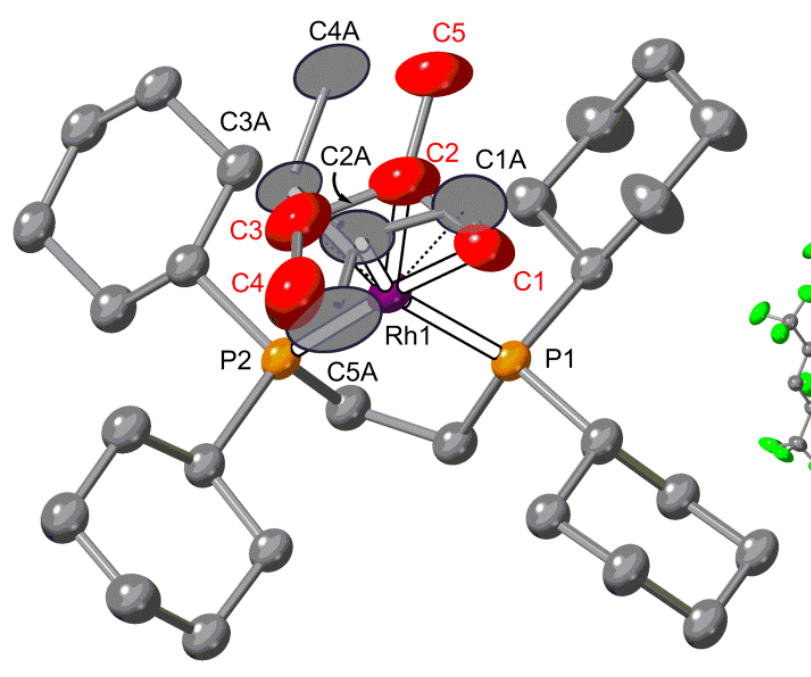

C

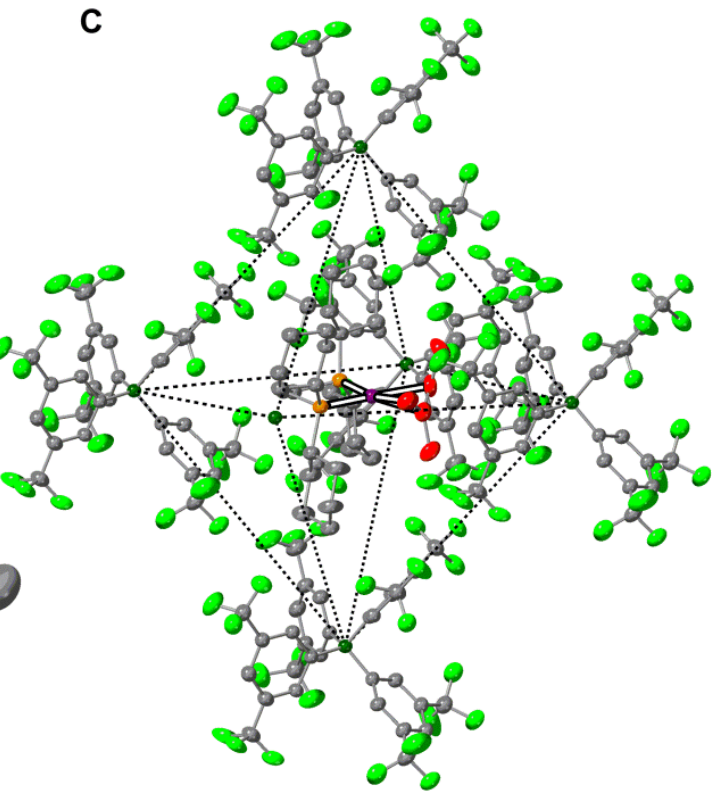

Figure S52: (A) Molecular structure of [1-methylbutenes][BAr ${ }_{4}$ ]. (B) Cationic fragment of [1-methylbutenes][BAr${ }_{4}$ ] showing two disorder components in 50: 50 ratio. One component has been solved as 2-methylbut-1-ene and the other 2-methylbut-2-ene. There was no significant difference in final R-value between solving either disorder component as either methylbutene isomer. (C) Structure displaying pseudo- $O_{\mathrm{h}}$ anion network of the $\left[\mathrm{BAr}{ }_{4}\right]^{-}$ ions, with cationic fragments sat within the cavity; $-\left(\mathrm{Ar}_{4}{ }_{4}\right)$ removed from 1 boron atom for clarity. Displacement ellipsoids set at $50 \%$ and hydrogen from phosphine ligand and $\left[\mathrm{BAr}{ }_{4}\right]^{-}$ anion removed for clarity in all parts.

Selected bond lengths ( $\AA$ ): Rh1-P1 2.2370(15), Rh1-P2 2.2314(16), Rh1-C1 2.23(2), Rh1C2 2.120(19), Rh1-C3 2.360(18), Rh1-C1A 2.41(3), Rh1-C2A 2.11(2), Rh1-C3A 2.27(16), C1-C2 1.39(3), C2-C3 1.52(3), C1A-C2A 1.54(3), C2A-C3A 1.37(3). 

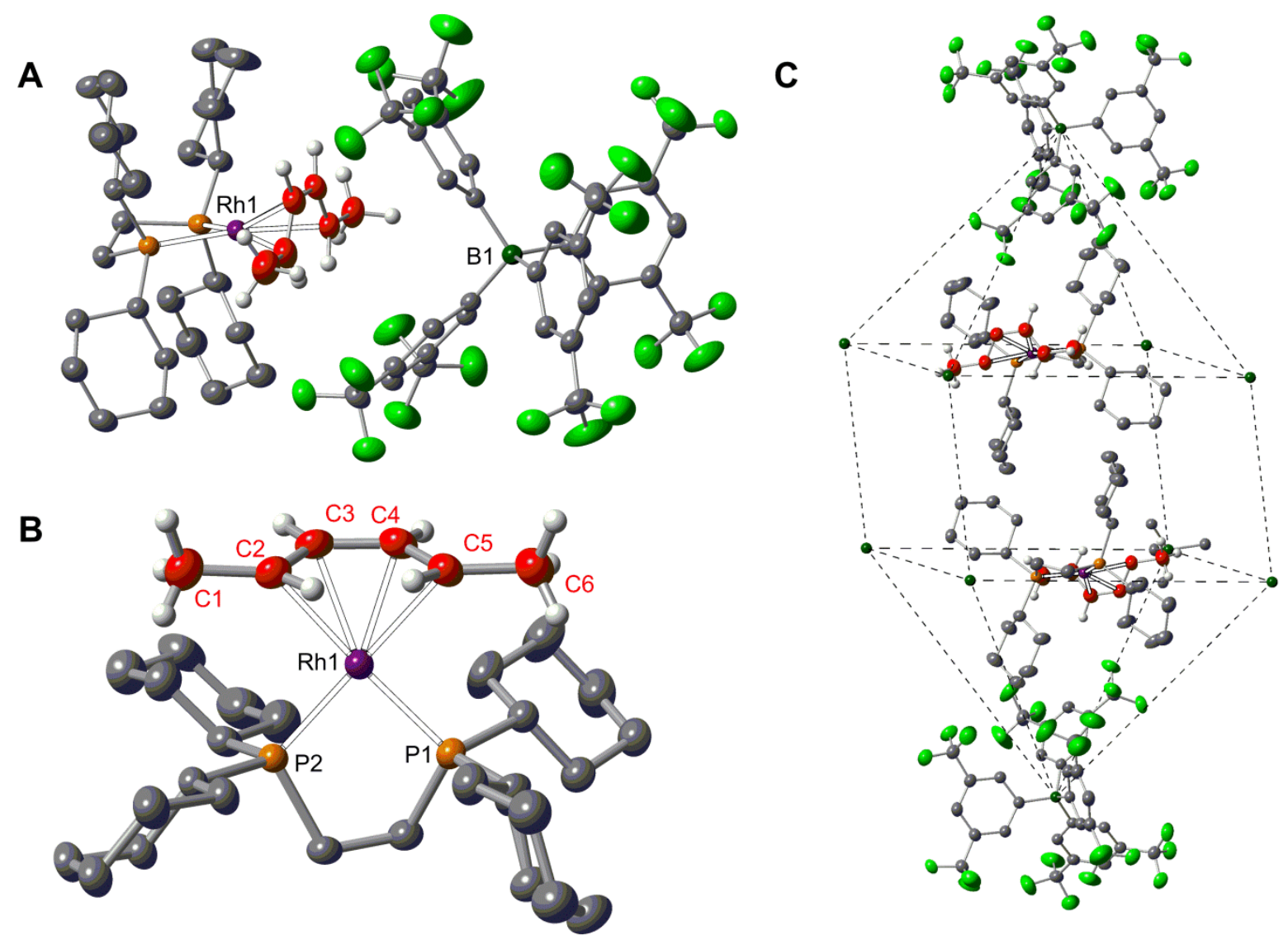

Figure S53: (A) Molecular structure of [1-hexadiene][BAr ${ }_{4}$ ]. (B) Cationic fragment of [1-hexadiene][BAr ${ }_{4}$ ] showing atom labelling. (C) Structure displaying bi-capped pyramidal anion network of the $\left[\mathrm{BAr}_{4}\right]^{-}$ions, with cationic fragments sat within the cavity; $-\left(\mathrm{Ar}_{4}\right)$ removed from 4 boron atoms for clarity. Displacement ellipsoids set at $50 \%$ and hydrogen from phosphine ligand and $\left[\mathrm{BAr}_{4}\right]^{-}$anion removed for clarity in all parts.

Selected bond lengths ( $\AA$ ): Rh1-P1 2.2755(9), Rh1-P2 2.2689(6), Rh1-C2 2.328(3), Rh1-C3 2.184(3), Rh1-C4 2.197(3), Rh1-C5 2.337(3), C2-C3 1.357(4), C4-C5 1.352(5). 
A
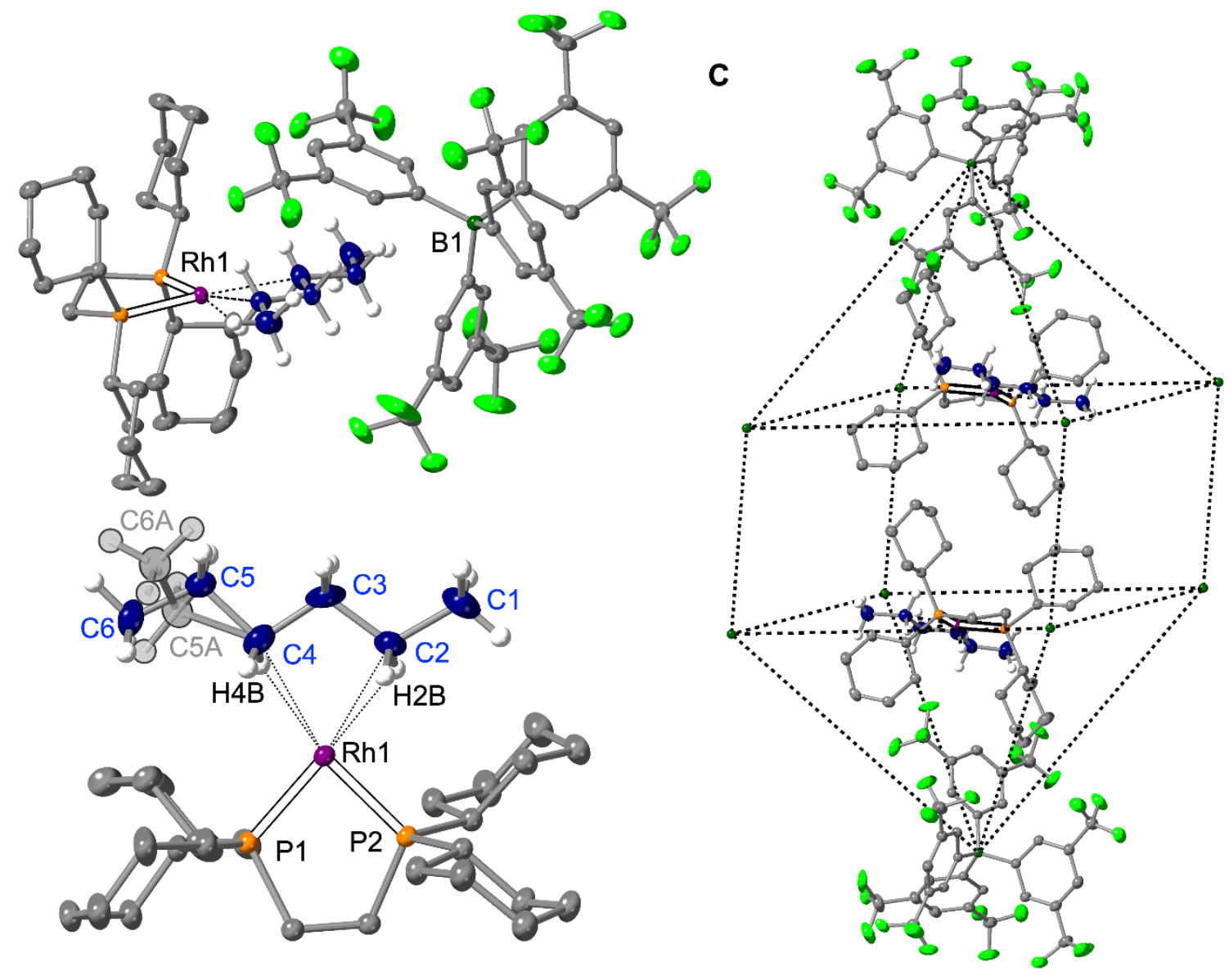

Figure S54: (A) Molecular structure of [1-hexane][BAr ${ }_{4}$ ]. (B) Cationic fragment of [1-hexane][BArF ${ }_{4}$ ] showing major disorder components, where the ratio of disorder components is 0.65 : 0.35 for major: minor. (C) Structure displaying bi-capped pyramidal anion network of the $\left[\mathrm{BAr}_{4}\right]^{-}$ions, with cationic fragments sat within the cavity; $-\left(\mathrm{Ar}_{4}{ }_{4}\right)$ removed from 4 boron atoms for clarity. Displacement ellipsoids set at $50 \%$ and hydrogen from phosphine ligand and $\left[\mathrm{BAr}_{4}\right]^{-}$anion removed for clarity in all parts.

Selected bond lengths ( $\AA$ ): Rh1-P1 2.2002(6), Rh1-P2 2.1910(6), Rh1-C2 2.527(3), Rh1-C4 2.549(4), Rh1-H2B 2.11(5), Rh1-H4B 2.14(5), Rh1-H2A 2.46(5), Rh1-H4A 2.37(5), C1-C2 1.544(15), C2-C3 1.511(5), C3-C4 1.504(16), C4-C5 1.528(6), C5-C6 1.460(8). 

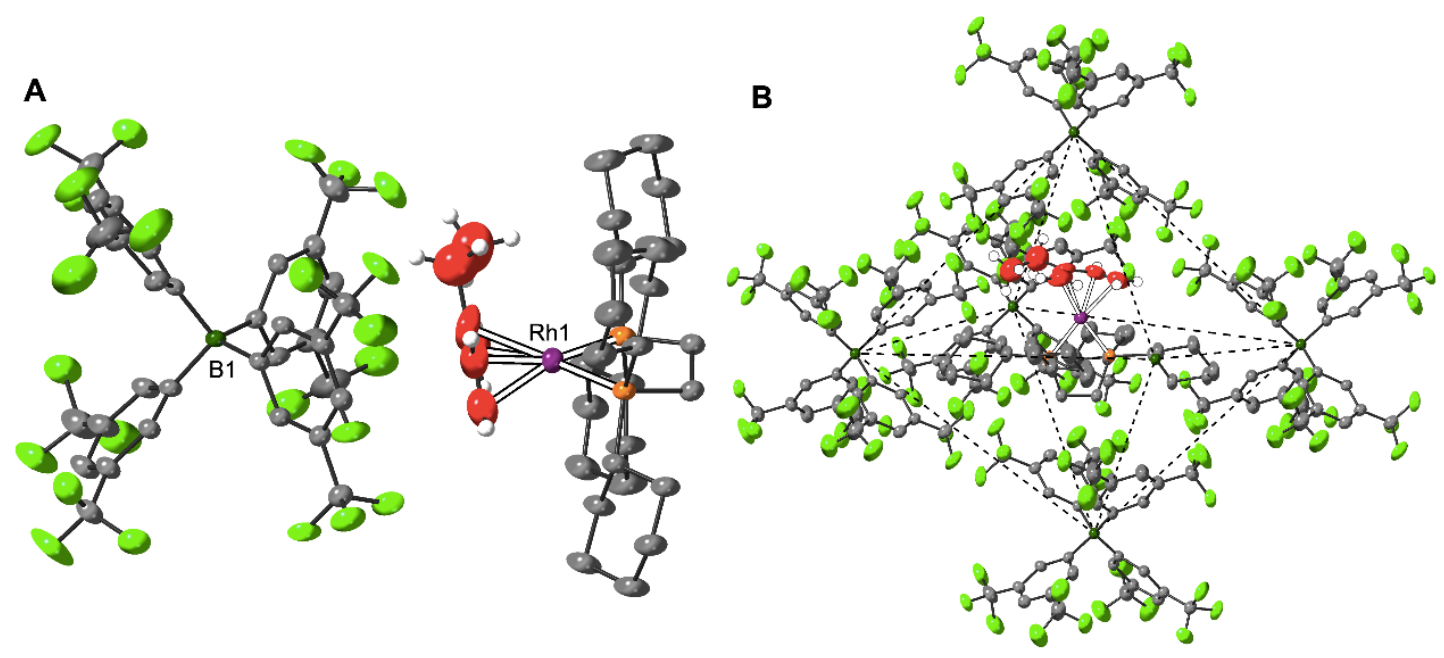

c

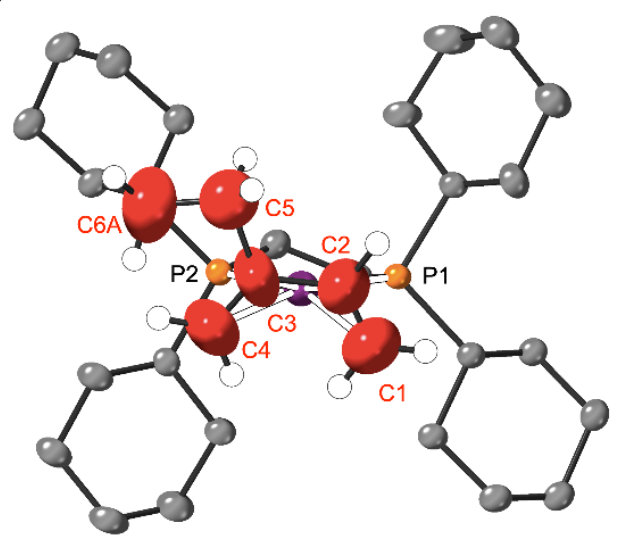

D

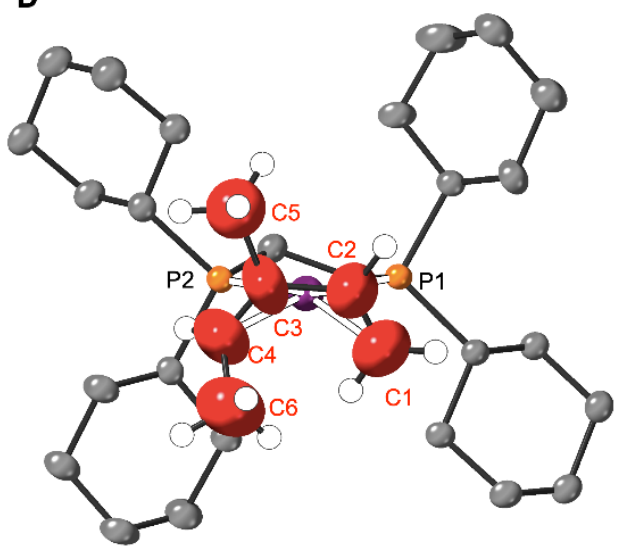

Figure S55: (A) Molecular structure of [1-( $\left.\left.\mathrm{C}_{6} \mathrm{H}_{10}\right)\right]\left[\mathrm{BAr}_{4}{ }_{4}\right]\left(\mathrm{C}_{6} \mathrm{H}_{10}=3\right.$-methyl-1,3-pentadiene or 2-ethylbutadiene). (B) pseudo- $O_{h}$ anion network of the $\left[\mathrm{BAr}_{4}\right]^{-}$ions, with cationic fragments sat within the cavity; $-\left(\mathrm{Ar}_{4}{ }_{4}\right)$ removed from 1 boron atom for clarity. (C) Cationic fragment of [1-( $\left.\left.\mathrm{C}_{6} \mathrm{H}_{10}\right)\right]\left[\mathrm{BAr}_{4}{ }_{4}\right]$ showing 2-ethylbutadiene coordinated isomer, and (D) showing 3-methyl-1,3-pentadiene coordinated isomer. These are solved as disorder components of one another, where the ratio of disorder components is 0.49: 0.51 ratio respectively. Displacement ellipsoids set at $50 \%$ and hydrogen from phosphine ligand and $\left[\mathrm{BAr}_{4}\right]^{-}$anion removed for clarity in all parts.

Selected bond lengths ( $\AA$ ): Rh1-P1 2.2628(12), Rh1-P2 2.2924(14), Rh1-C1 2.237(10), Rh1C2 2.101(8), Rh1-C3 2.161(7), Rh1-C4 2.287(9), C1-C2 1.278(14), C2-C3 1.503(14), C3-C4 1.245(13), C3-C5 1.502(17), C4-C6 1.37(3), C5-C6A 1.48(3). 

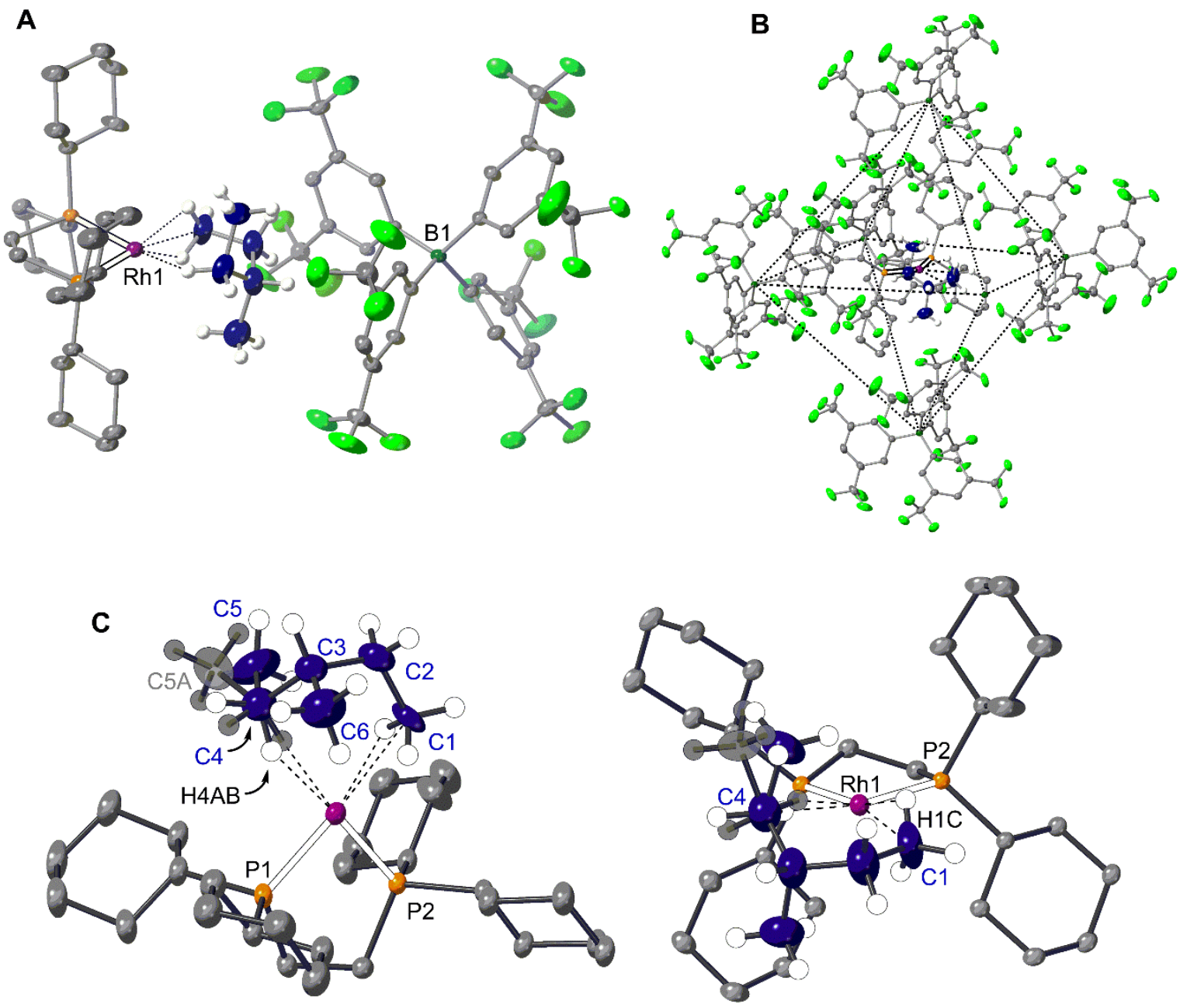

Figure S56: (A) Molecular structure of [1-(3-methylpentane)][BAr $\left.{ }_{4}\right]$. (B) Structure displaying pseudo- $\mathrm{O}_{\mathrm{h}}$ anion network of the $\left[\mathrm{BAr}_{4}\right]^{-}$ions, with cationic fragments sat within the cavity; $-\left(\mathrm{Ar}_{4}{ }_{4}\right)$ removed from 1 boron atom for clarity. (C) Cationic fragment of [1-(3-methylpentane)][BArF ${ }_{4}$ ] showing atom labelling. showing major disorder components, where the ratio of disorder components is 0.81: 0.19 for major: minor. Displacement ellipsoids set at $50 \%$ and hydrogen from phosphine ligand and $\left[\mathrm{BAr}_{4}{ }_{4}\right]^{-}$anion removed for clarity in all parts.

Selected bond lengths ( $\AA$ ): Rh1-P1 2.1965(7), Rh1-P2 2.1959(7), Rh1-C1 2.430(4), Rh1-C4 2.788(6), Rh1-H4AB 1.9196(4), C1-C2 1.504(7), C3-C4 1.475(8). 


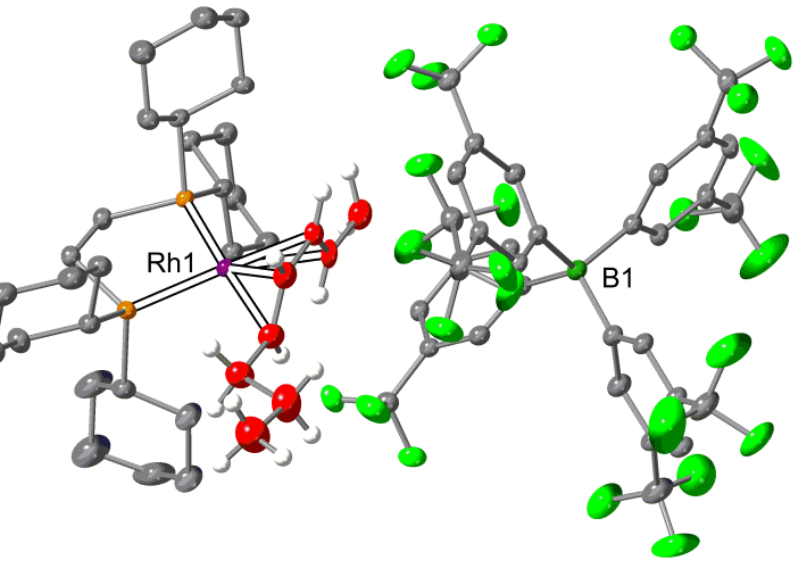

B

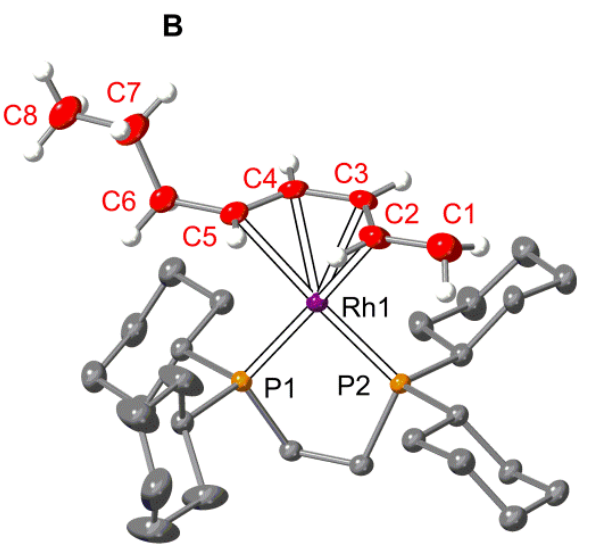

C

D

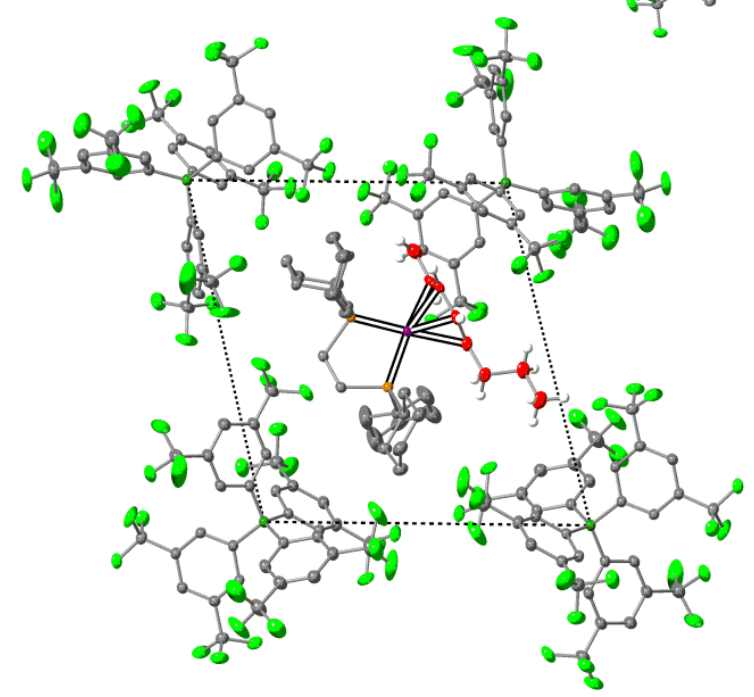

Figure S57: (A) Molecular structure of [1-octadiene][BAr ${ }_{4}$ ]. (B) Cationic fragment of [1-octadiene][BAr $\left.{ }_{4}\right]$ showing atom labelling. (C) Structure displaying pseudo- $O_{\mathrm{h}}$ anion network of the $\left[\mathrm{BAr}_{4}\right]^{-}$ions, with cationic fragments sat within the cavity; - $\left(\mathrm{Ar}_{4}{ }_{4}\right)$ removed from 1 boron atom for clarity. (D) Top view of pseudo- $O_{h}$ anion-cage, showing twisted cation orientation. Displacement ellipsoids set at $50 \%$ and hydrogen from phosphine ligand and $\left[\mathrm{BAr}_{4}\right]^{-}$anion removed for clarity in all parts.

Selected bond lengths ( $\AA$ ): Rh1-P1 2.2874(6), Rh1-P2 2.2627(6), Rh1-C2 2.259(3), Rh1-C3 2.175(3), Rh1-C4 2.201(3), Rh1-C5 2.359(3), C2-C3 1.363(5), C4-C5 1.340(5). 


\section{S.3.5. Comparison of Rh $\cdots C$ distances from selected $\sigma$-alkane complexes}

Table S2: Comparison of $R h \cdots C$ distances in $\left[R h\left(L_{2}\right)(\right.$ alkane $\left.)\right]\left[\right.$ anion], $L_{2}=$ chelating phosphine.

\begin{tabular}{|c|c|c|c|}
\hline Complex & 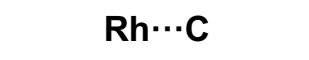 & Hapacity & Ref \\
\hline$\left[\mathrm{Rh}\left({ }^{\mathrm{i}} \mathrm{Bu}_{2} \mathrm{PCH}_{2} \mathrm{CH}_{2} \mathrm{P}^{\mathrm{i}} \mathrm{Bu} \mathrm{u}_{2}\right)(\mathrm{NBA})\right]\left[\mathrm{BAr}_{4}\right]$ & $\begin{array}{l}2.494(10), \\
2.480(11)\end{array}$ & $1,2-\eta^{2}, \eta^{2}$ & S20 \\
\hline$\left[\mathrm{Rh}\left(\mathrm{Cy}_{2} \mathrm{PCH}_{2} \mathrm{CH}_{2} \mathrm{PCy}\right)(\mathrm{NBA})\right]\left[\mathrm{BAr}_{4}\right]$ & $2.389(3), 2.400(3)$ & $1,2-\eta^{2}, \eta^{2}$ & S2 \\
\hline$\left[\mathrm{Rh}\left(\mathrm{Cy}_{2} \mathrm{PCH}_{2} \mathrm{CH}_{2} \mathrm{PCy}\right)(\right.$ pentane $\left.)\right]\left[\mathrm{BAr}_{4}\right]$ & $2.514(4), 2.522(5)$ & $2,4-\eta^{2}, \eta^{2}$ & S1 \\
\hline$\left[\mathrm{Rh}\left(\mathrm{Cyp}_{2} \mathrm{PCH}_{2} \mathrm{CH}_{2} \mathrm{PCyp} 2\right)(\mathrm{NBA})\right]\left[\mathrm{BAr}_{4}\right]$ & $2.388(5), 2.392(5)$ & $1,2-\eta^{2}, \eta^{2}$ & $\mathrm{~S} 21$ \\
\hline$\left[\mathrm{Rh}\left(\mathrm{Cy}_{2} \mathrm{PCH}_{2} \mathrm{CH}_{2} \mathrm{PCy}\right)(\mathrm{NBA})\right]\left[\mathrm{BAr}_{4}\right]$ & $2.387(5), 2.378(5)$ & $1,2-\eta^{2}, \eta^{2}$ & $\mathrm{~S} 22$ \\
\hline$\left[\mathrm{Rh}\left(\mathrm{Cy}_{2} \mathrm{PCH}_{2} \mathrm{CH}_{2} \mathrm{CH}_{2} \mathrm{PCy}\right)(\mathrm{NBA})\right]\left[\mathrm{BAr}_{4}\right]$ & $2.408(2), 2.402(2)$ & $1,2-\eta^{2}, \eta^{2}$ & \multirow{3}{*}{$\mathrm{S} 23$} \\
\hline$\left[\mathrm{Rh}\left(\mathrm{Cy}_{2} \mathrm{PCH}_{2} \mathrm{CH}_{2} \mathrm{CH}_{2} \mathrm{CH}_{2} \mathrm{PCy}\right)(\mathrm{NBA})\right]\left[\mathrm{BAr}_{4}\right]$ & 2.399(2), 2.396(2) & $1,2-\eta^{2}, \eta^{2}$ & \\
\hline$\left[\mathrm{RhH}\left(\mathrm{Cy}_{2} \mathrm{PCH}_{2} \mathrm{CHCH}_{2} \mathrm{CH}_{2} \mathrm{PCy}_{2}\right)(\mathrm{NBA})\right]\left[\mathrm{BArF}_{4}\right]$ & $2.90(3)$ & $\eta^{1}$ & \\
\hline$\left[\mathrm{Rh}\left(\mathrm{Cy}_{2} \mathrm{PCH}_{2} \mathrm{CH}_{2} \mathrm{PCy}\right)(\right.$ cyclohexane $\left.)\right]\left[\mathrm{BAr}_{4}\right]$ & $2.62(2), 2.53(2)$ & $\begin{array}{l}\text { Between } 1,3- \\
\eta^{2}, \eta^{2} \text { and } \\
1,3-\eta^{1}, \eta^{1}\end{array}$ & \multirow[t]{2}{*}{ S7 } \\
\hline$\left[\mathrm{Rh}\left(\mathrm{Cy}_{2} \mathrm{PCH}_{2} \mathrm{CH}_{2} \mathrm{PCy}\right)_{2}\right.$ (isobutane $\left.)\right]\left[\mathrm{BAr}_{4}\right]$ & $\begin{array}{c}2.362(14) \\
2.443(7) \\
\end{array}$ & $1,2-\eta^{2}, \eta^{2}$ & \\
\hline$\left[\mathrm{Rh}\left(\mathrm{Cy}_{2} \mathrm{PCH}_{2} \mathrm{CH}_{2} \mathrm{PCy}_{2}\right)(\right.$ propane $\left.)\right]\left[\mathrm{BAr}_{4}\right]$ & $2.46(2), 2.46(2)$ & $1,3-\eta^{2}, \eta^{2}$ & \multirow{4}{*}{$\begin{array}{l}\text { This } \\
\text { work }\end{array}$} \\
\hline$\left[\mathrm{Rh}\left(\mathrm{Cy}_{2} \mathrm{PCH}_{2} \mathrm{CH}_{2} \mathrm{PCy}_{2}\right)(\right.$ hexane $\left.)\right]\left[\mathrm{BAr}_{4}\right]$ & $2.527(3), 2.549(4)$ & $2,4-\eta^{2}, \eta^{2}$ & \\
\hline$\left[\mathrm{Rh}\left(\mathrm{Cy}_{2} \mathrm{PCH}_{2} \mathrm{CH}_{2} \mathrm{PCy}\right)(2-\right.$ methylbutane $\left.)\right]\left[\mathrm{BAr}_{4}\right]$ & 2.348(9), 2.39(1) & $1,2-\eta^{2}, \eta^{2}$ & \\
\hline$\left[\mathrm{Rh}\left(\mathrm{Cy}_{2} \mathrm{PCH}_{2} \mathrm{CH}_{2} \mathrm{PCy}_{2}\right)(3\right.$-methylpentane $\left.)\right]\left[\mathrm{BAr}_{4}\right]$ & $2.430(4), 2.788(6)$ & $1,4-\eta^{2}, \eta^{1}$ & \\
\hline
\end{tabular}

Note: The complexes presented in Table $S 2$ are formed with a pseudo- $O_{h}$ arrangement of $\left[\mathrm{BAr}_{4}\right]^{-}$anions, with the exception of $\left[\mathrm{Rh}\left(\mathrm{Cy}_{2} \mathrm{PCH}_{2} \mathrm{CH}_{2} \mathrm{PCy}_{2}\right)(\right.$ pentane $\left.)\right]\left[\mathrm{BAr}_{4}\right]$ and $\left[\mathrm{Rh}\left(\mathrm{Cy}_{2} \mathrm{PCH}_{2} \mathrm{CH}_{2} \mathrm{PCy}_{2}\right)\right.$ (hexane)][BAr$\left.{ }_{4}\right]$ which have a bi-capped square pyramidal arrangement, and $\left[\mathrm{Rh}\left(\mathrm{Cy}_{2} \mathrm{PCH}_{2} \mathrm{CH}_{2} \mathrm{PCy}_{2}\right)(\mathrm{NBA})\right]\left[\mathrm{BAr}_{4}\right]$ where eight $\left.\left[\mathrm{BAr}_{4}\right]_{4}\right]^{-}$anions form a gyrobifastigium arrangement made of two, face-sharing, parallelepipeds. 


\section{S.4. Computational Methods}

\section{S.4.1. Solid State Calculations}

All static Kohn-Sham DFT calculations were performed on periodic models of the studied rhodium complexes, employing the Gaussian Plane Wave (GPW) formalism as implemented in the QUICKSTEP ${ }^{\mathrm{S} 24}$ module within the CP2K program suite (Version 5.0). ${ }^{\mathrm{S} 25}$ Molecularly optimised basis sets of double- $\zeta$ quality plus polarization in their short-range variant (DZVPMOLOPT-SR-GTH) ${ }^{\mathrm{S} 26}$ were used on all atomic species. The interaction between the core electrons and the valence shell (Rh: 17, B: 3, C: 4, P: 5, F: 7, H: 1 electrons) was described by Goedecker-Teter-Hutter (GTH) pseudo potentials. ${ }^{\text {S27-29 }}$ The generalized gradient approximation (GGA) to the exchange-correlation functional according to Perdew-BurkeErnzerhof $(\mathrm{PBE})^{\mathrm{S} 30}$ was used in combination with Grimme's D3-correction for dispersion interactions. ${ }^{\text {S31 }}$ The auxiliary plane wave basis set was truncated at a cutoff of 500 Ry. The maximum force convergence criterion was set to $10^{-4} \mathrm{Eh} \cdot \mathrm{Bohr}^{-1}$, whilst default values were used for the remaining criteria. The convergence criterion for the self-consistent field (SCF) accuracy was set to $10^{-7}$ Eh and $10^{-8}$ Eh for geometry optimizations and vibrational analysis, respectively.

The Brillouin zone was sampled using the $\Gamma$-point. Initial coordinates were obtained from the experimental crystallographic data, with the hydrogen positions normalised where possible with Mercury. ${ }^{\mathrm{S32}}$ Periodic boundary conditions (PBC) were applied throughout in combination with fixed unit cell parameters obtained from experiment. All geometries were first partially relaxed, keeping the heavy atoms (non-H, F) fixed, then fully relaxed without imposing any constraints, whilst keeping unit cell parameters constant in all cases.

All optimised stationary points were further characterized by analysis of their numerical second derivatives with a displacement of $0.01 \mathrm{Bohr}$ and were found to have no imaginary eigenvalues.

Gibbs free energies for structures computed in the solid state were calculated using the TAMkin software toolkit. ${ }^{533}$ Numerical instability when recomputing the wavefunction of the alternative rotamer of 1-(3-methylpentane)][BAr ${ }_{4}$ ] prevented us from defining a free energy for this species and so $\triangle S C F$ energies are quoted in Sections 2.1.5 and 2.1.7 for [1-(2-methylbutane)][BAr$\left.{ }_{4}\right]$ and 1-(3-methylpentane)][BAr ${ }_{4}$ ] respectively. In the former case, a free energy difference of $+10.3 \mathrm{kcal} / \mathrm{mol}$ is computed, $1.5 \mathrm{kcal} / \mathrm{mol}$ higher than the $\triangle S C F$ energy. Based on this the $\triangle S C F$ value of $2.5 \mathrm{kcal} / \mathrm{mol}$ quoted for 1-(3methylpentane)][BAr ${ }_{4}$ ] is likely to be underestimated by a similar amount.

The CIFs of the fully optimised systems, generated using VESTA ${ }^{\mathrm{S} 34}$, were analysed using the Crystal Explorer package ${ }^{\mathrm{S} 35}$, using a central cation and the six nearest neighbour anions. 
The cation with its six neighbouring anions were generated with the following method:

- Open the CIF in CrystalExplorer.

- Select the cation of interest, Select Display -> Invert Selection and then Remove -> Selected Atoms.

- Select Actions - > Generate Atoms Within Radius.

- Enter an appropriate radius, (around $11 \AA$ from the central cation in our case).

- Select the surrounding anions and the central cation and then select Remove -> "Incomplete Fragments".

- Select all remaining atoms and then Actions-> "Complete Fragments".

- Select the central cation then Actions -> "Generate Surface" to generate the Hirshfeld Surface.

- Modify the charges and multiplicities of all the fragments as required.

All computed structures are available as a separate file of Cartesian coordinates.

\section{S.4.2. Molecular Calculations}

Molecular calculations employed the GAUSSIAN 09 (revision D.01) program package ${ }^{\mathrm{S} 36}$ and employed the BP86 GGA functional. ${ }^{\mathrm{S} 7-38}$ Stuttgart-Dresden (SDD) ${ }^{\mathrm{S} 39}$ relativistic effective core potentials (ECP) in combination with the associated basis sets were utilized to describe Rh and $P$, with polarization functions added for $P(\zeta=0.387) .{ }^{540}$ The $6-31 G(d, p)$ basis sets $^{\text {s41-42 }}$ were used on remaining atoms.

Electronic structure analyses were performed on the geometries of the Rh cations extracted from the CP2K-optimised structures with the heavy atoms fixed at the experimental positions. An electron density file suitable for further analysis was generated from a singlepoint calculation.

The topology of the electron density was analysed by means of QTAIM (Quantum Theory of Atoms in Molecules), ${ }^{\$ 43}$ as implemented in the AIMALL package. ${ }^{\$ 44}$ Inner shell electrons on $\mathrm{Rh}$ and $\mathrm{P}$ modelled by ECPs were represented by core density functions (extended wavefunction format). All bond critical bond (BCPs) are shown in green, ring critical points (RCPs) in pink. Contours plots are in the plane containing $\mathrm{Rh}$ and the two $\mathrm{H}$ atoms involved in the $\sigma$-interactions.

NBO calculations were performed using the NBO 6.0 program $^{\mathrm{S45}}$, using the same geometries as for the QTAIM calculations above. Donor-acceptor interactions are presented 
in the main text as a sum of all contributions to a given type of pairing (e.g. all $\mathrm{Rh}_{\mathrm{LP}} \rightarrow \sigma^{*}{ }^{*}-\mathrm{H}$ donations above the threshold of $0.05 \mathrm{kcal} / \mathrm{mol}$ ). All individual donor-acceptor pairs are depicted for [1-propane] ${ }^{+}$(see Figures S68 to S75) and these are illustrative of the donoracceptors interactions in the other systems. The latter are tabulated with additional figures presented when specifically referred to in the main text.

$\mathrm{NCl}$ calculations were performed using the NCIPLOT program, ${ }^{544-47}$ using the nearest neighbour ion-pair molecular structures extracted from CP2K optimised geometries. The promolecular electron density was employed and isosurfaces are generated for $s=0.3$ au and $-0.07 \leq \rho \leq 0.07$ au.

Orbital plots were created with Chemcraft ${ }^{\$ 48}$ with an outer contour value of 0.07465 .

Data from the partially optimised (with non- $\mathrm{H}$ and $\mathrm{F}$ atoms fixed at their crystallographically determined positions) and the fully optimised structures (used in the final analyses) are presented below side by side for comparison. 


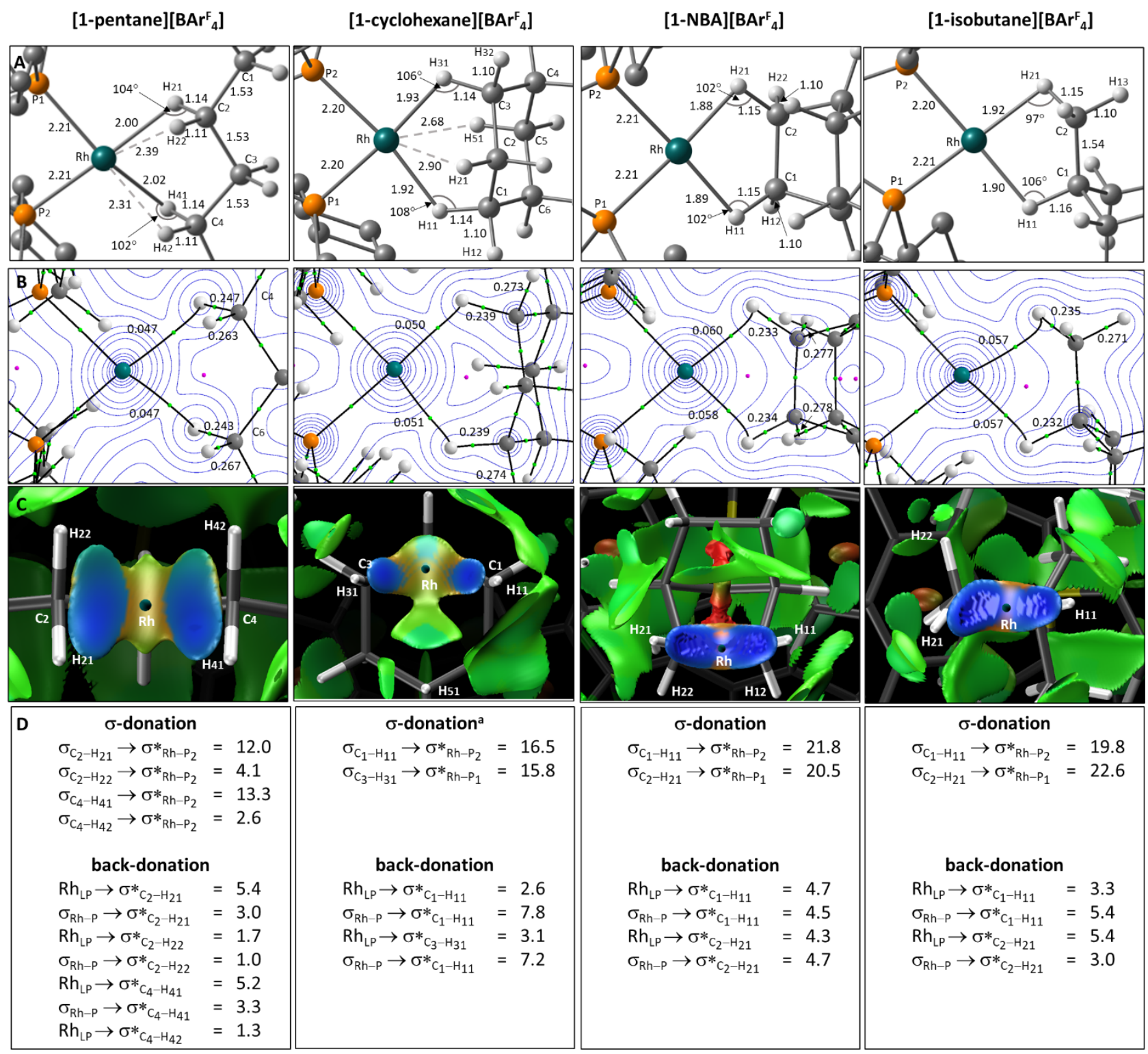

Figure S58: (A) Computed structure of the [1-alkane] ${ }^{+}$cations not presented in the main paper highlighting key distances $(\AA)$ and angles to $\mathrm{H}$ atoms. Non-alkane $\mathrm{H}$ atoms are omitted for clarity (B) QTAIM molecular graphs (C) Detail of the $\mathrm{NCl}$ plots viewed from the Rh center looking down an axis passing through the center of the alkane moiety interacting with Rh. (D) Major donoracceptor interactions derived from a $2^{\text {nd }}$ order perturbation NBO analysis ( $\mathrm{kcal} / \mathrm{mol}$ ) Values are the sum of each type of donor-acceptor interactions (see tables below for full details). 


\section{S.5. Electronic Structure Analysis}

S.5.1. [1-propane][BAr ${ }_{4}$ ]

\section{[1-propane][BArF $\left.{ }_{4}\right]$ (Partially Optimised)}

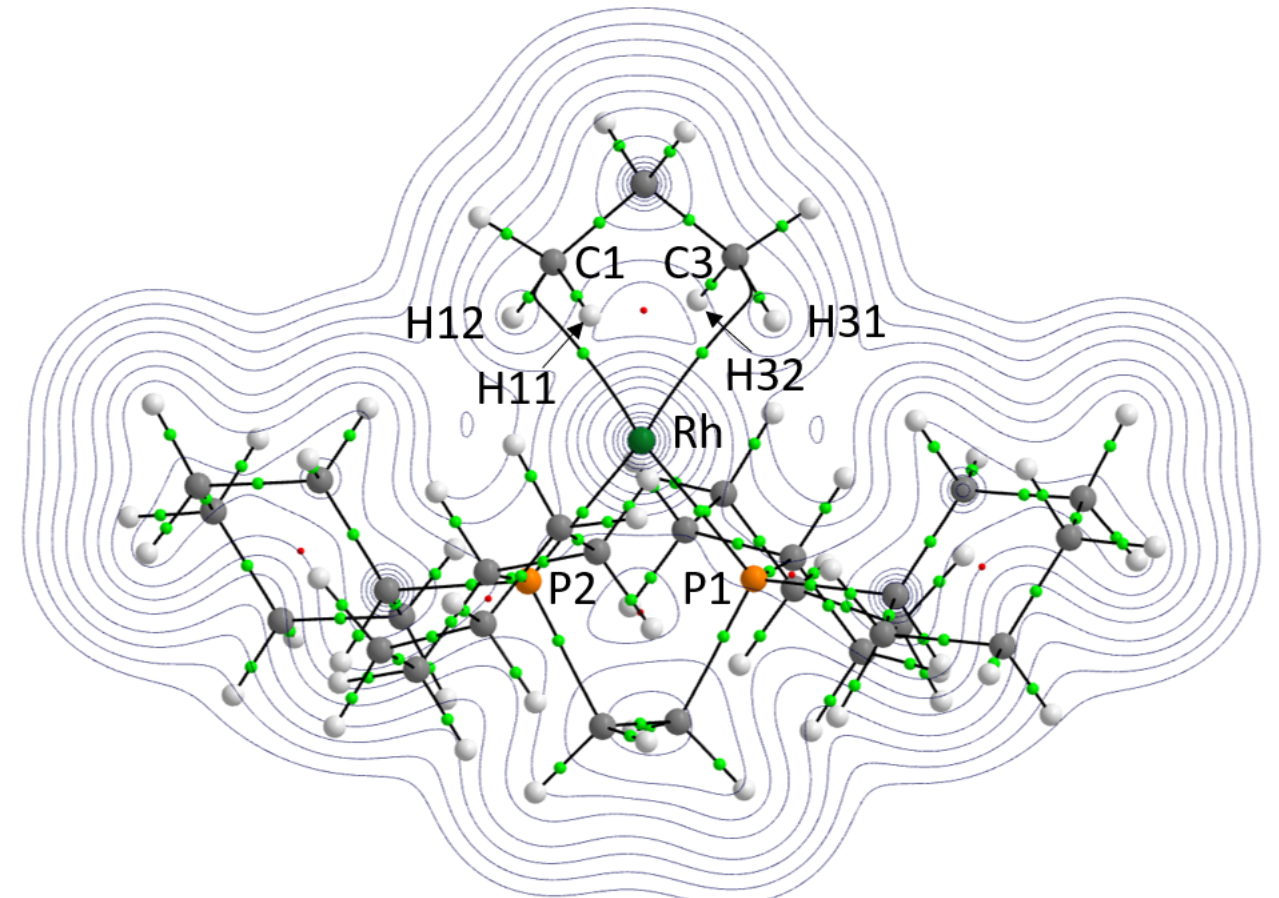

Figure S59: QTAIM molecular graph of [1-propane][BAr ${ }_{4}$ ] (partially optimised) with electron density contours in the H12-Rh-H31 plane.
Table S3: QTAIM BCP data and bond lengths for key bonds.

\begin{tabular}{|c|c|c|c|c|}
\hline & Interatomic & & & \\
\hline $\begin{array}{l}\text { Bond } \\
\text { Path }\end{array}$ & $\begin{array}{c}\text { Distance } \\
(\AA)\end{array}$ & $\begin{array}{c}\rho \\
\left(\mathrm{e} b o h r^{-3}\right)\end{array}$ & $\begin{array}{c}\nabla^{2} \rho \\
\left(e b^{-5}\right)\end{array}$ & $H(r)$ \\
\hline $\mathrm{C} 1-\mathrm{H} 12$ & 1.128 & 0.2505 & -0.7428 & -0.2339 \\
\hline $\mathrm{H} 12-\mathrm{Rh}$ & 2.074 & & No BCP & \\
\hline C1-Rh & 2.451 & 0.0447 & 0.1851 & -0.0015 \\
\hline Rh-P2 & 2.206 & 0.1191 & 0.0727 & -0.0591 \\
\hline $\mathrm{C} 3-\mathrm{H} 31$ & 1.126 & 0.2518 & -0.7541 & -0.2364 \\
\hline C3-Rh & 2.462 & 0.0436 & 0.1798 & -0.0013 \\
\hline H31-Rh & 2.089 & & No BCP & \\
\hline Rh-P1 & 2.227 & 0.1151 & 0.0792 & -0.0546 \\
\hline
\end{tabular}




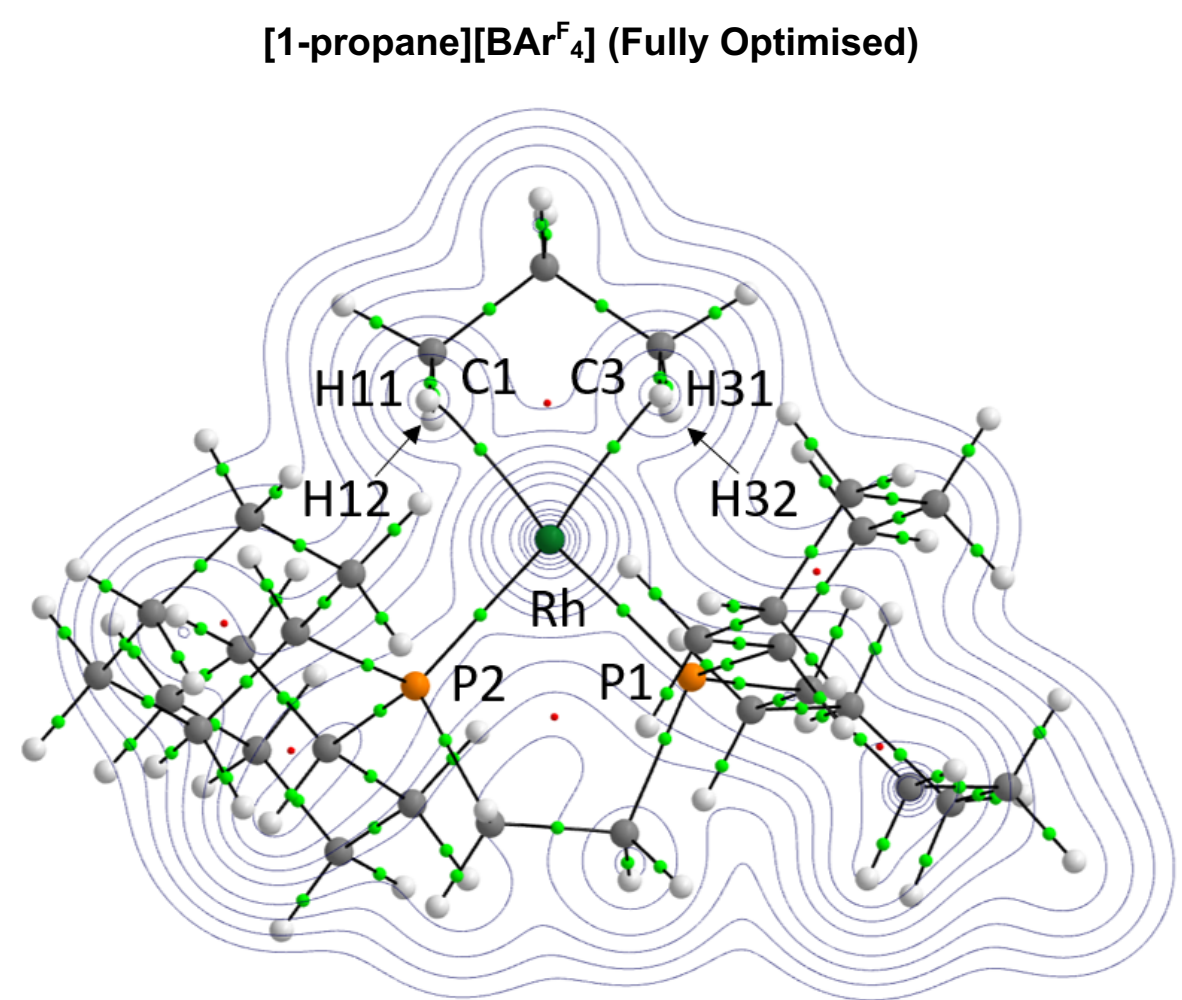

Table S4: QTAIM BCP data and bond lengths for key bonds.

\begin{tabular}{|c|c|c|c|c|}
\hline & Interatomic & & & \\
\hline $\begin{array}{l}\text { Bond } \\
\text { Path }\end{array}$ & $\begin{array}{c}\text { Distance } \\
(\AA)\end{array}$ & $\begin{array}{c}\rho \\
\left(e^{e} b o h r^{-3}\right)\end{array}$ & $\begin{array}{c}\nabla^{2} \rho \\
\left(\text { e bohr } r^{-5}\right)\end{array}$ & $H(r)$ \\
\hline $\mathrm{C} 1-\mathrm{H} 11$ & 1.134 & 0.2463 & -0.7123 & -0.2262 \\
\hline $\mathrm{H} 11-\mathrm{Rh}$ & 2.027 & 0.0457 & 0.1764 & -0.0039 \\
\hline $\mathrm{C} 1-\mathrm{Rh}$ & 2.504 & & No $B C P$ & \\
\hline $\mathrm{Rh}-\mathrm{P} 2$ & 2.207 & 0.1193 & 0.0706 & -0.0594 \\
\hline $\mathrm{C} 3-\mathrm{H} 31$ & 1.136 & 0.2449 & -0.7026 & -0.2241 \\
\hline $\mathrm{H} 31-\mathrm{Rh}$ & 2.001 & 0.0476 & 0.1799 & -0.0049 \\
\hline C3-Rh & 2.506 & & No BCP & \\
\hline Rh-P1 & 2.205 & 0.1198 & 0.0688 & -0.0598 \\
\hline
\end{tabular}

Figure S60: QTAIM molecular graph of [1-propane][BAr ${ }_{4}$ ] (fully optimised) with electron density contours in the H11-Rh-H31 plane. 

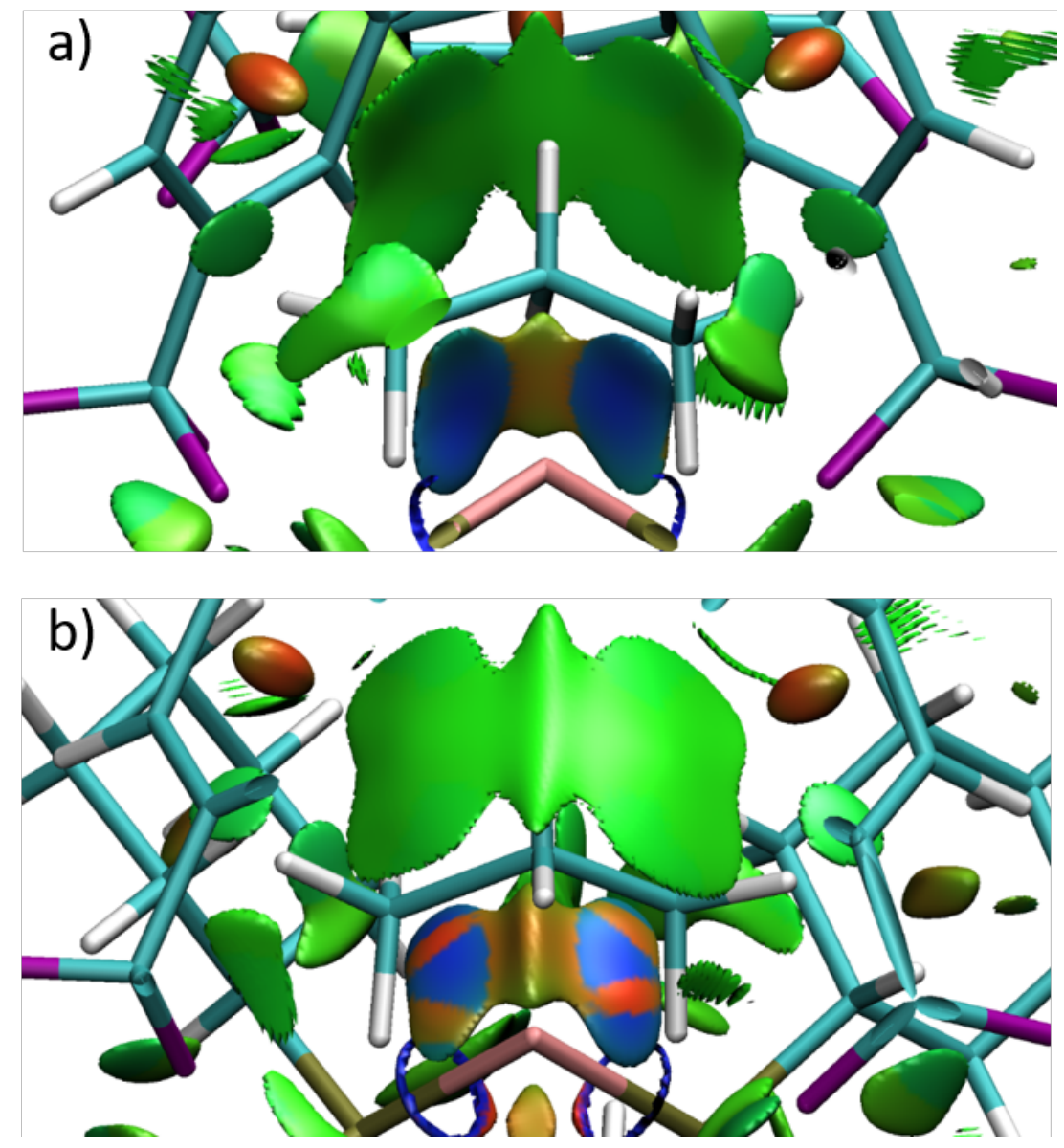

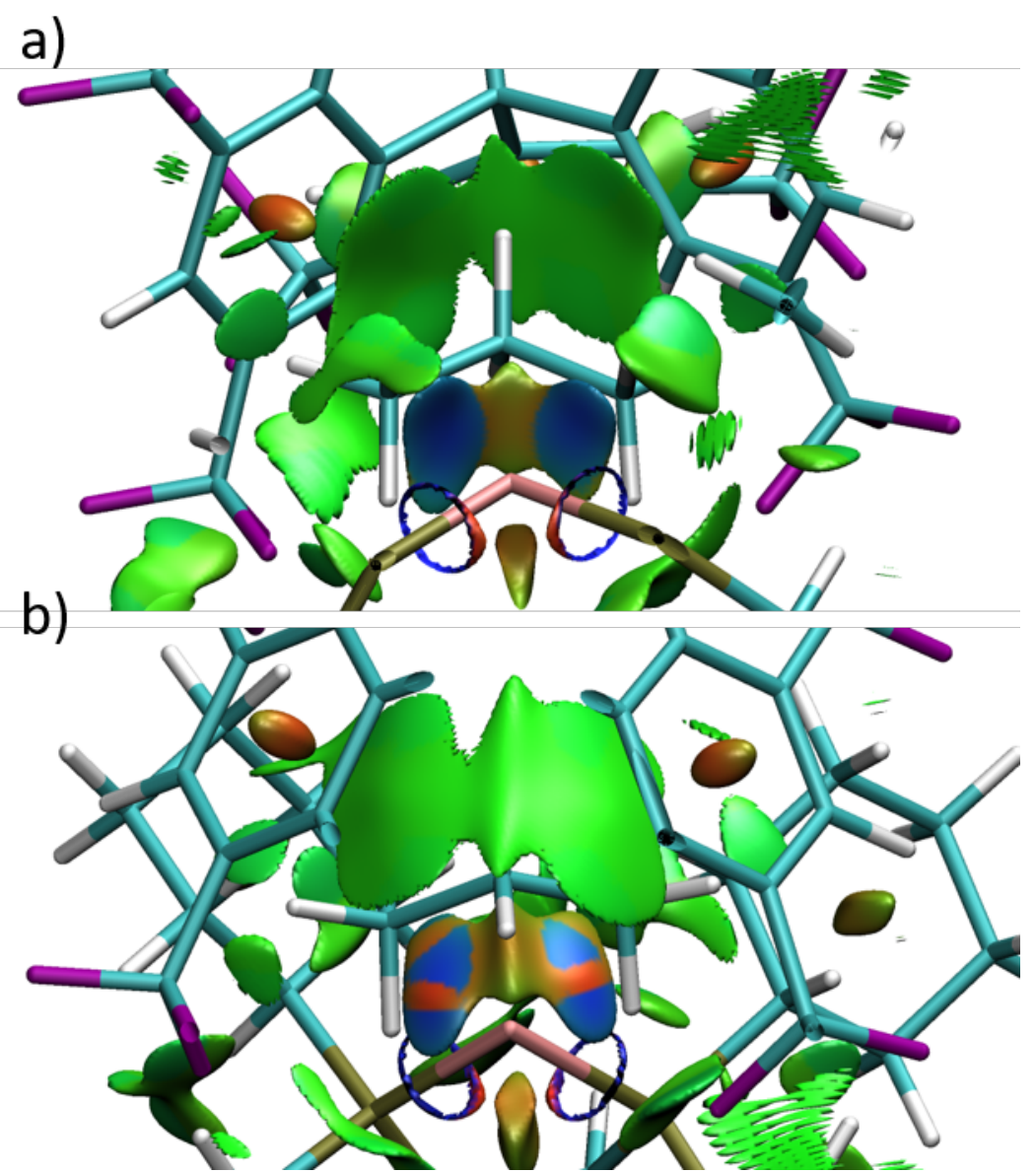

Figure S61: a) $\mathrm{NCl}$ Plot of [1-propane][BArF ${ }_{4}$ ] (partially optimised) with Figure S62: a) NCl Plot of [1-propane][BAr ${ }_{4}$ ] (fully optimised) with isosurfaces generated for $s=0.3$ au and $-0.07<\rho<0.07$ au. View from Rh isosurfaces generated for $s=0.3$ au and $-0.07<\rho<0.07$ au. View from Rh looking towards the alkane. b) View from above the alkane looking towards looking towards the alkane. b) View from above the alkane looking towards $\mathrm{Rh}$.
$\mathrm{Rh}$. 

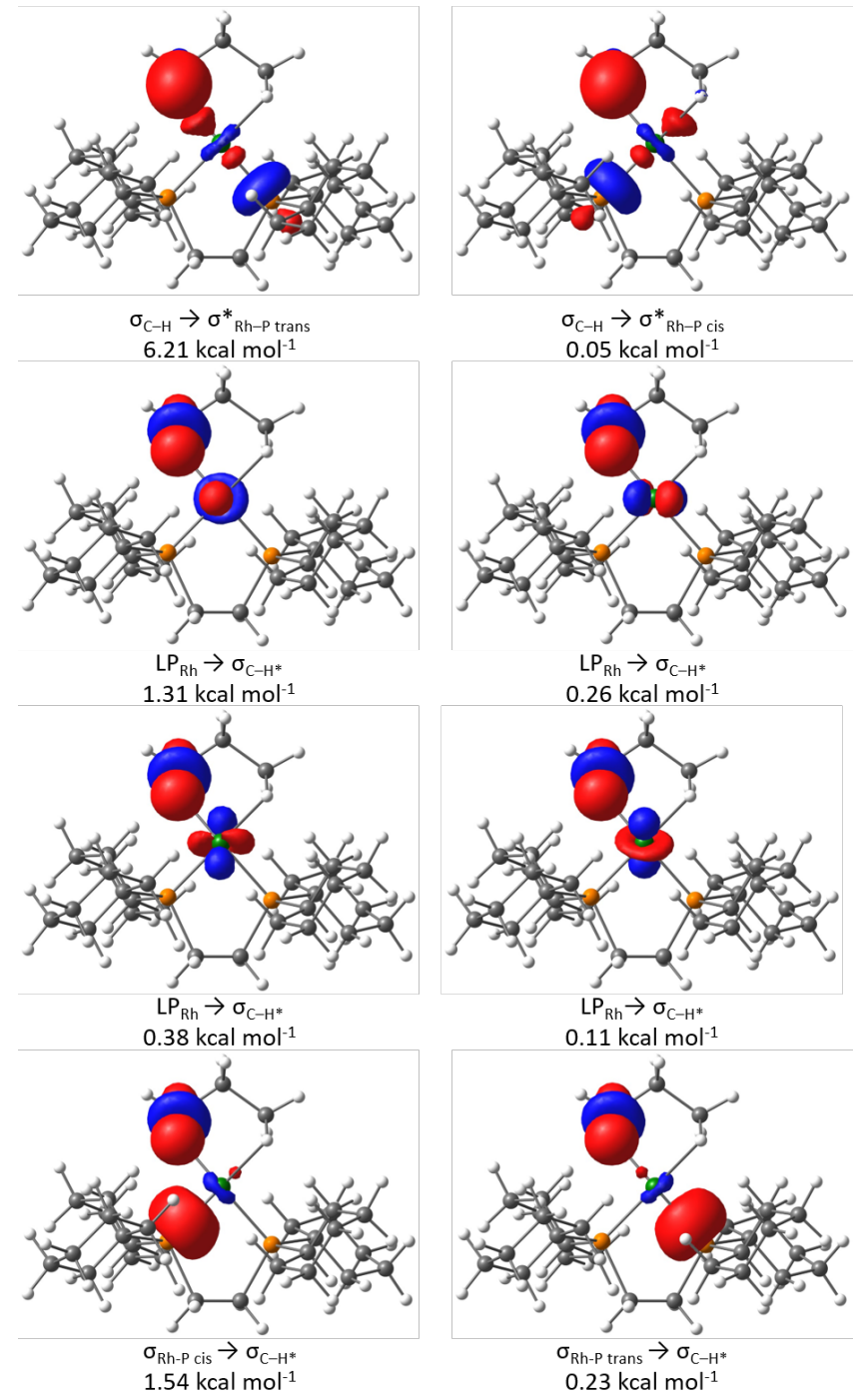
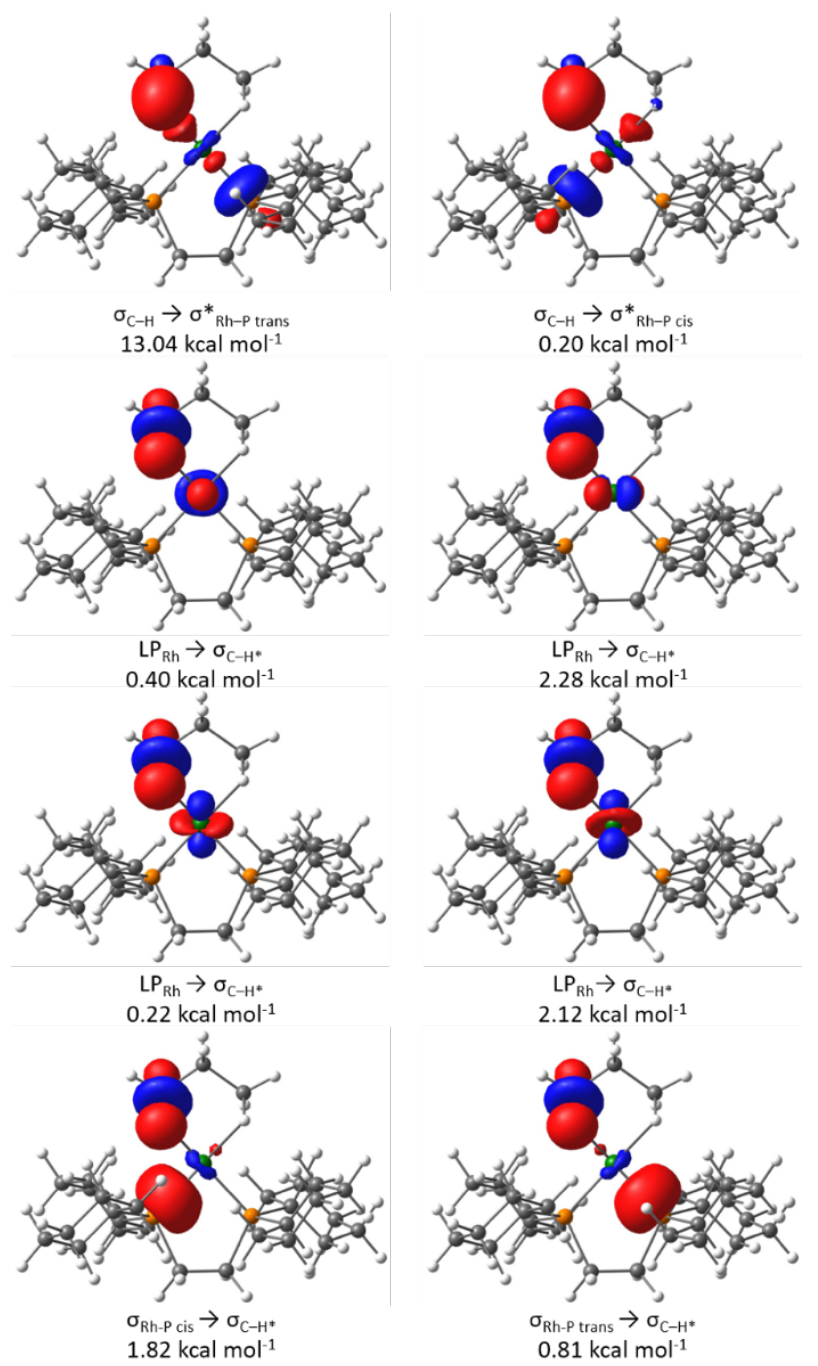

Figure S63: NBO donor acceptor orbital pairs of $\mathrm{C} 3-\mathrm{H} 31$ in [1-propane][BArF 4 ] Figure S64: NBO donor acceptor orbital pairs of C3-H31 in [1-propane][BArF ${ }_{4}$ (fully (partially optimised) with their associated $2^{\text {nd }}$ order perturbation theory energies. 

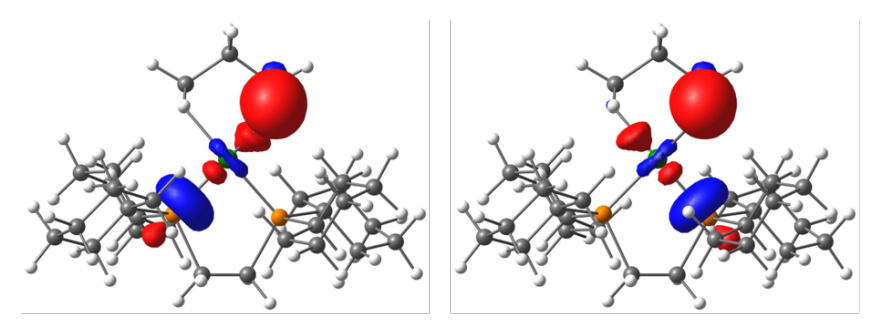

$\sigma_{\mathrm{C}-\mathrm{H}} \rightarrow \sigma^{*}{ }_{\mathrm{Rh}-\mathrm{P} \text { tran }}$
$10.60 \mathrm{kcal} \mathrm{mol}^{-1}$

$\sigma_{C-H} \rightarrow \sigma^{*}{ }_{\text {Rh-P cis }}$
$0.09 \mathrm{kcal} \mathrm{mol}^{-1}$
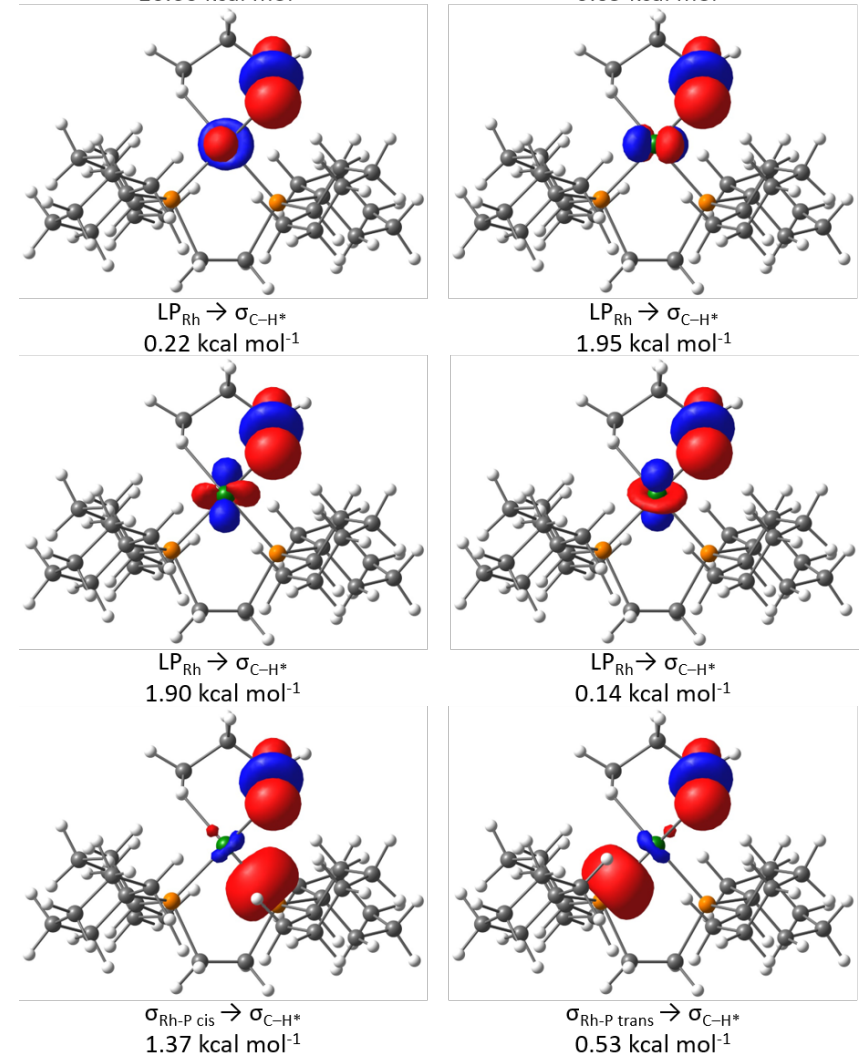
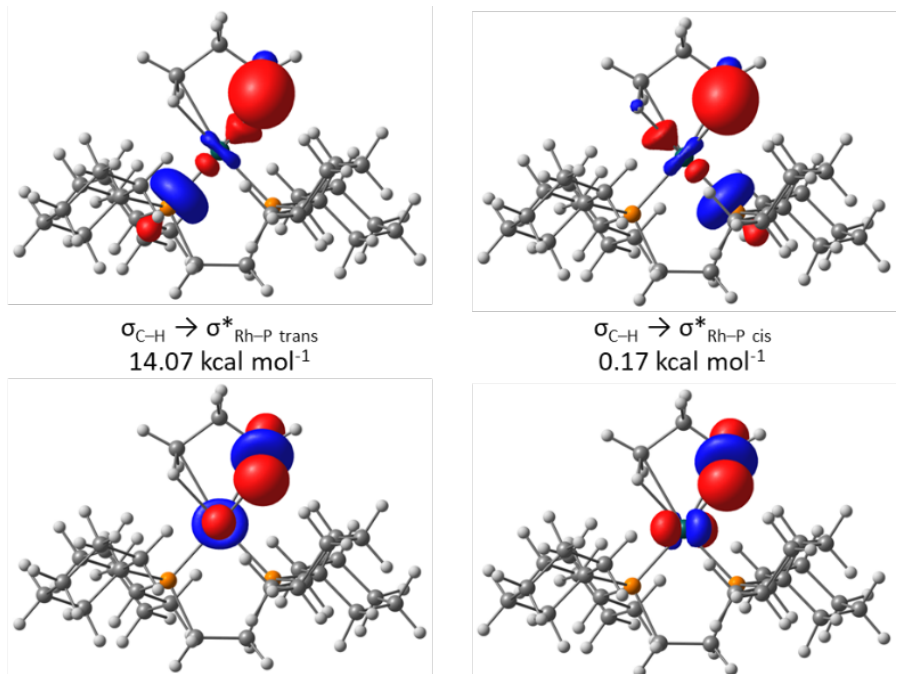

$\mathrm{LP}_{\mathrm{Rh}} \rightarrow \sigma_{\mathrm{C}-\mathrm{H}^{*}}$

$0.72 \mathrm{kcal} \mathrm{mol}^{-1}$

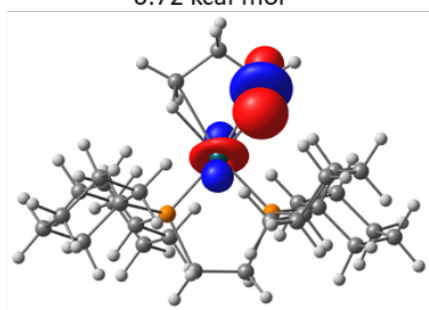

$\mathrm{LP}_{\mathrm{Rh}} \rightarrow \sigma_{\mathrm{C}-\mathrm{H}^{*}}$

$3.03 \mathrm{kcal} \mathrm{mol}^{-1}$
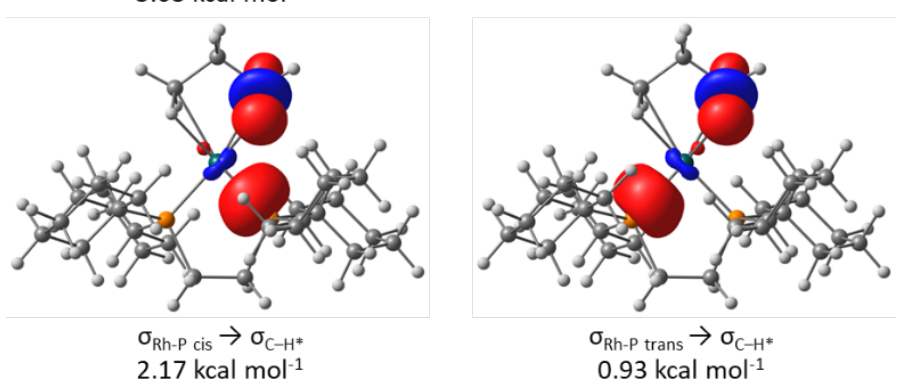

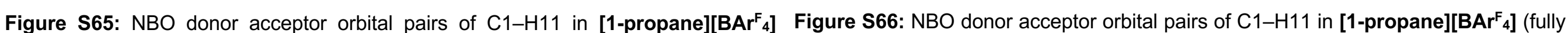
(partially optimised) with their associated $2^{\text {nd }}$ order perturbation theory energies optimised) with their associated $2^{\text {nd }}$ order perturbation theory energies 


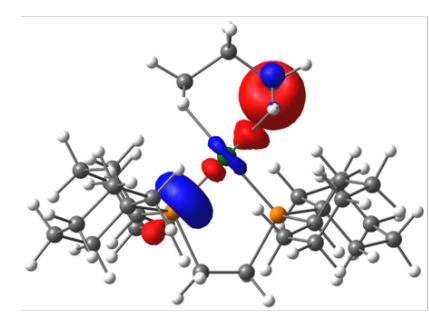

$\sigma_{C-H} \rightarrow \sigma^{*}$

$6.23 \mathrm{kcal} \mathrm{mol}^{-1}$
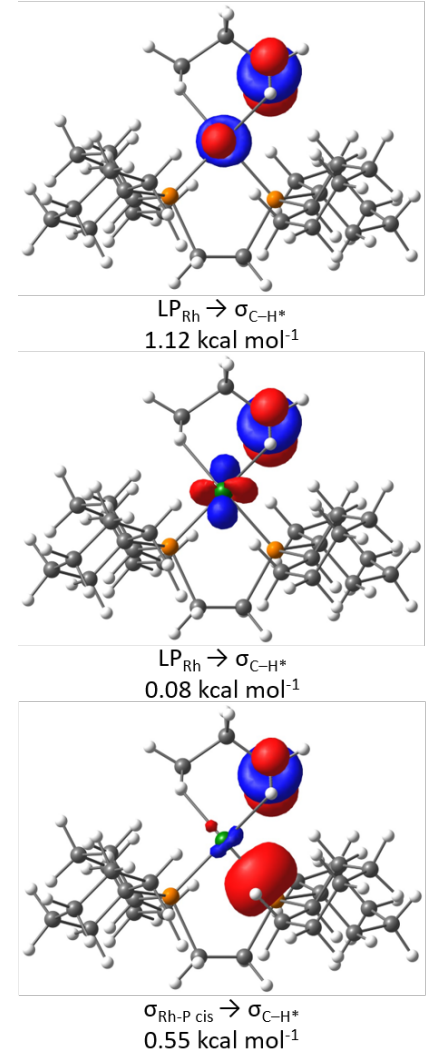

$0.55 \mathrm{kcal} \mathrm{mol}^{-1}$
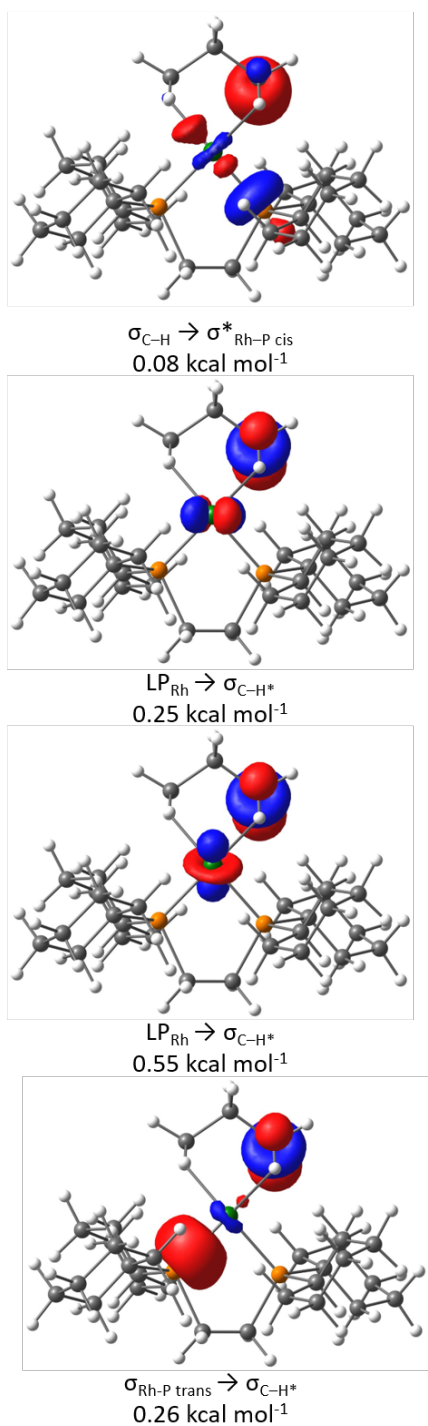
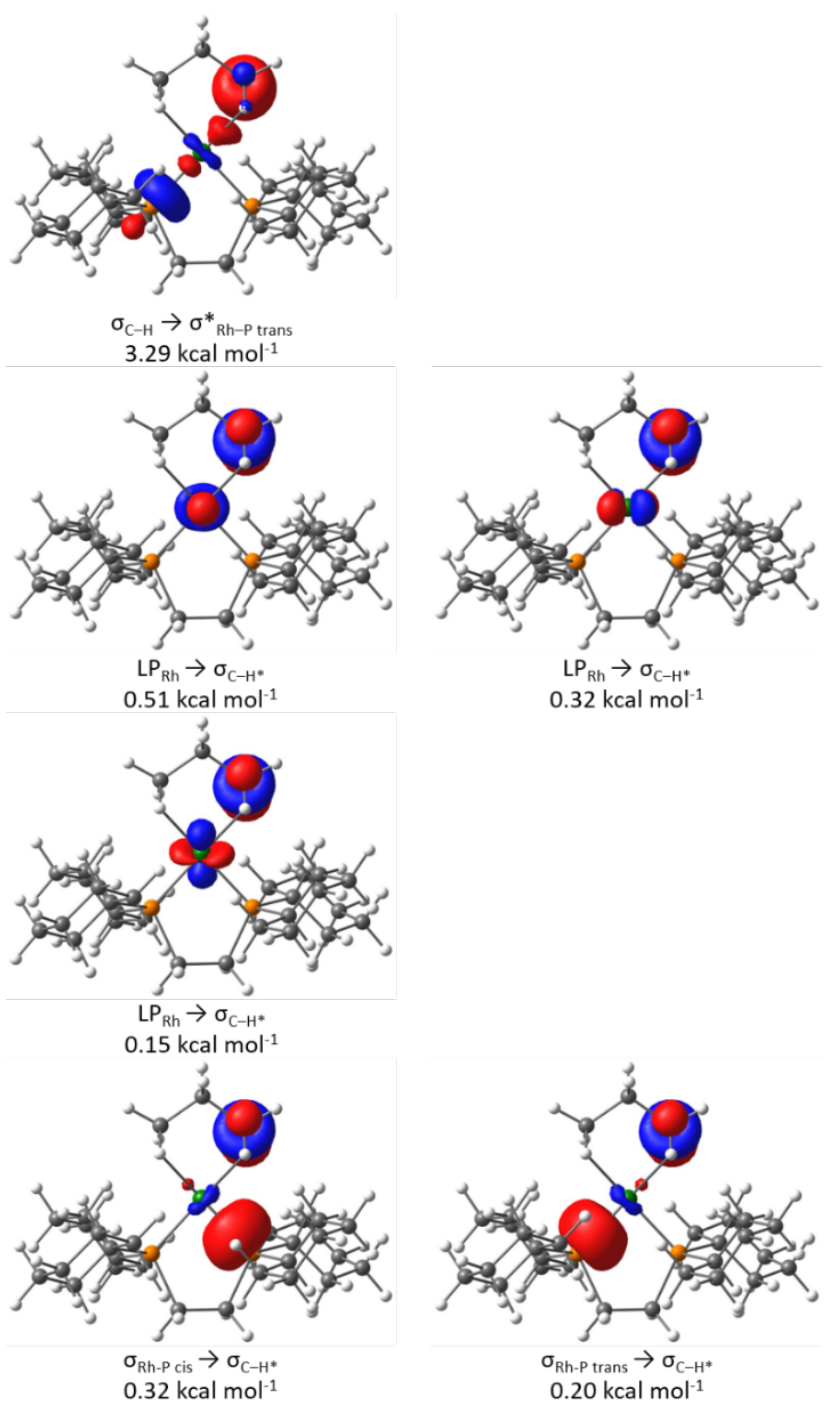

Figure S68: NBO donor acceptor orbital pairs of $\mathrm{C} 1-\mathrm{H} 12$ in [1-propane][BArF ${ }_{4}$ ] (fully optimised) with their associated $2^{\text {nd }}$ order perturbation theory energies.
Figure S67: NBO donor acceptor orbital pairs of $\mathrm{C} 1-\mathrm{H} 12$ in [1-propane][BArF ${ }_{4}$ ] (partially optimised) with their associated $2^{\text {nd }}$ order perturbation theory energies. 

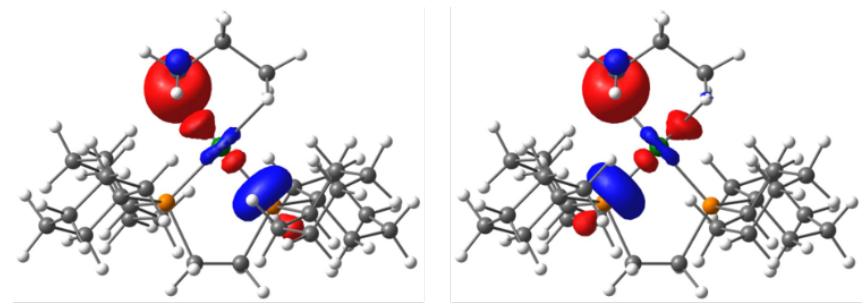

$\sigma_{\mathrm{CH}} \rightarrow \sigma_{\text {Rh-P trats }}$

$\sigma_{\mathrm{C}-\mathrm{H}} \rightarrow \sigma^{*}{ }_{\mathrm{Rh}-\mathrm{P} \text { cis }}$
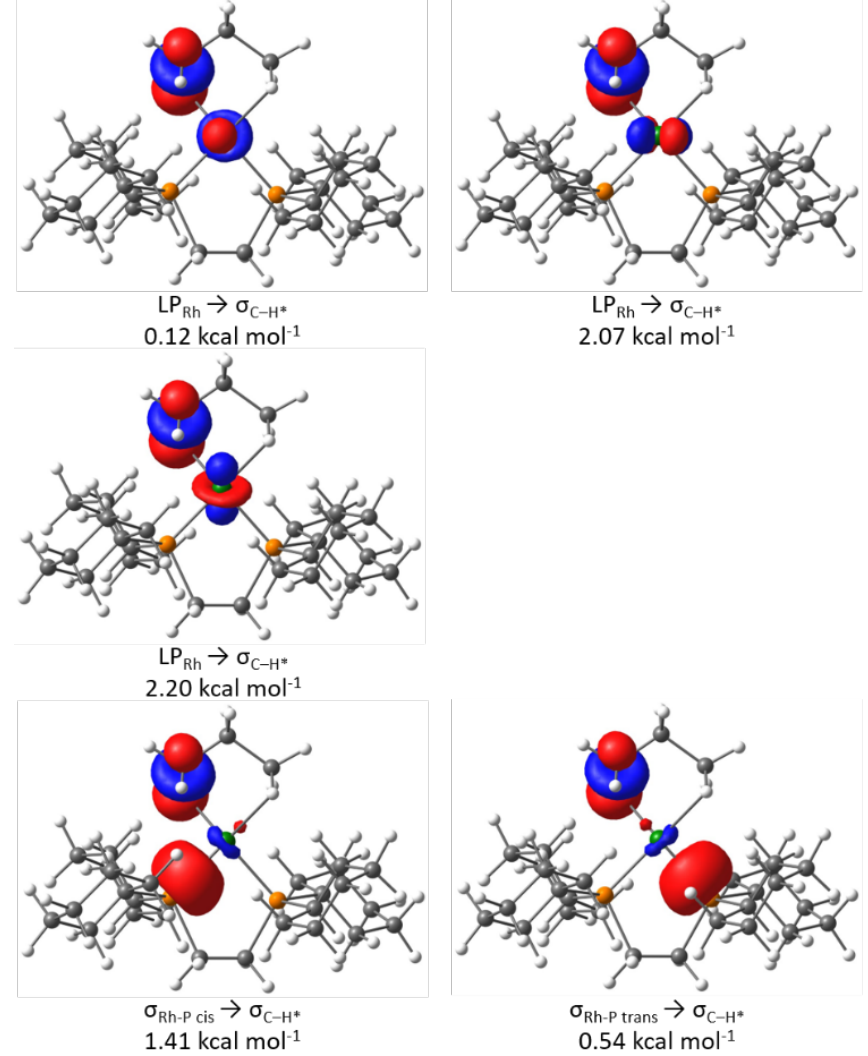

Figure S69: NBO donor acceptor orbital pairs of $\mathrm{C} 3-\mathrm{H} 32$ in [1-propane][BArF $\left.\mathrm{B}_{4}\right]$ (partially optimised) with their associated $2^{\text {nd }}$ order perturbation theory energies.
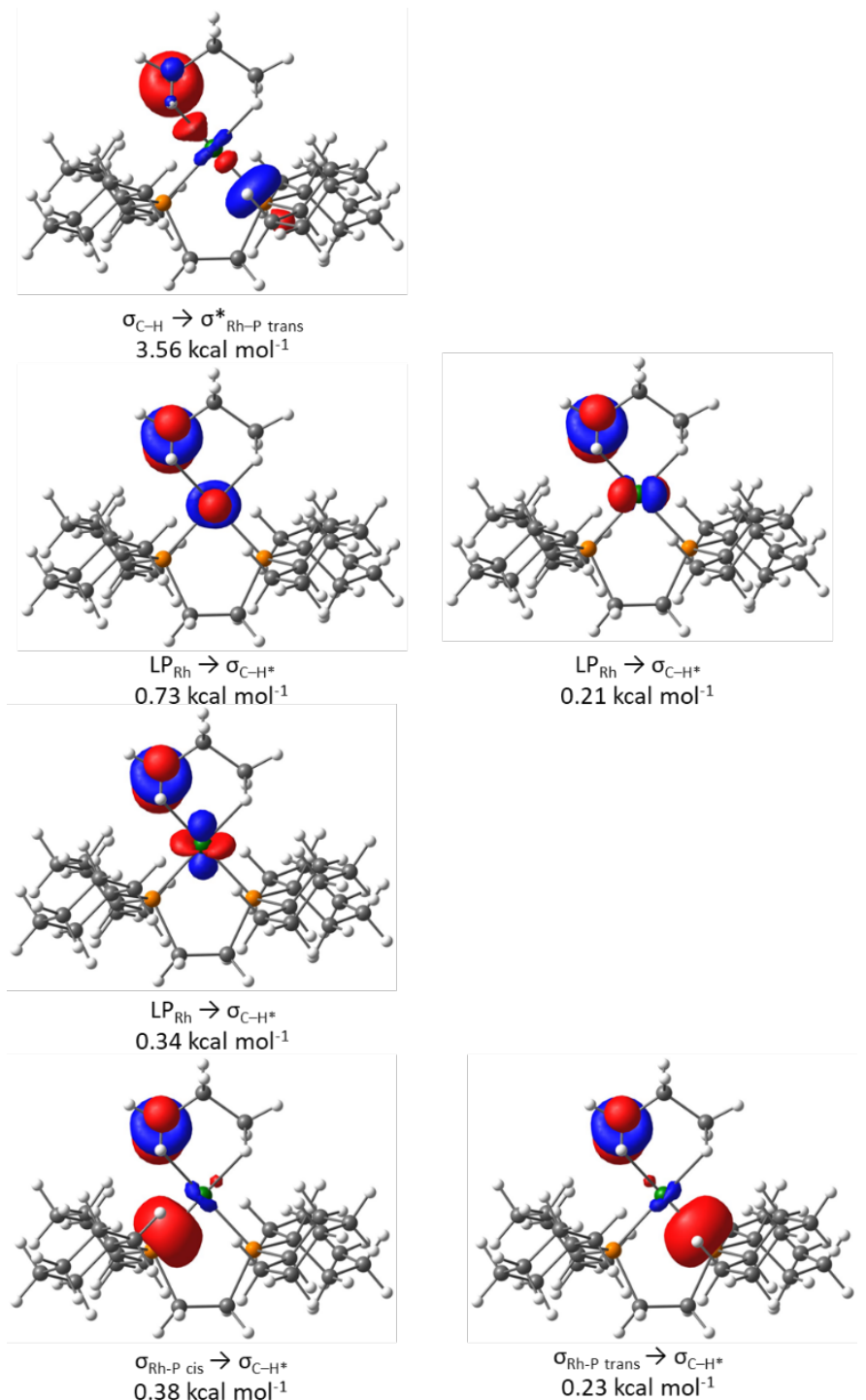

Figure S70: NBO donor acceptor orbital pairs of $\mathrm{C} 3-\mathrm{H} 32$ in [1-propane][BArF ${ }_{4}$ ] (fully optimised) with their associated $2^{\text {nd }}$ order perturbation theory energies. 

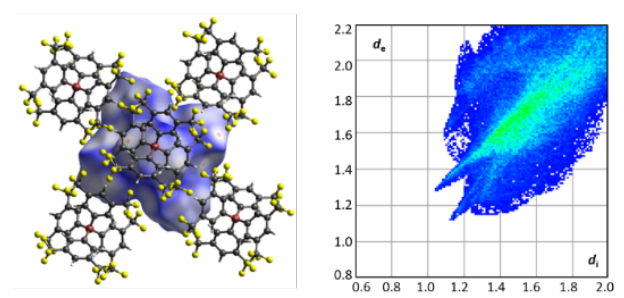

Full System

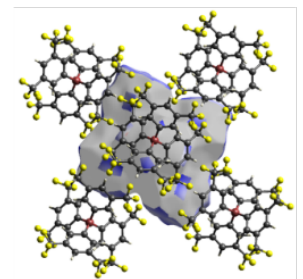

$\mathrm{H} \cdots \mathrm{H}(24.3 \%)$
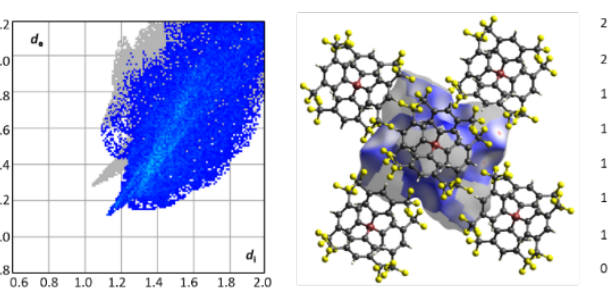

$\mathrm{H} \cdots \mathrm{F}(63.2 \%)$

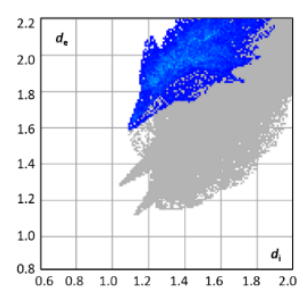

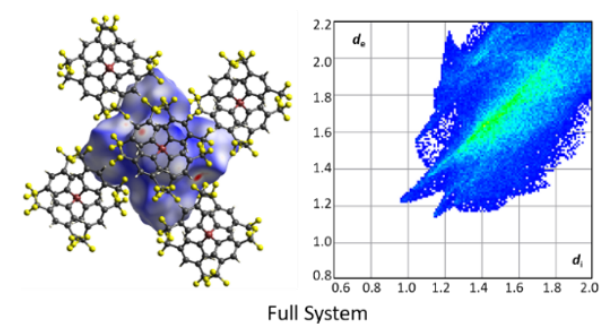
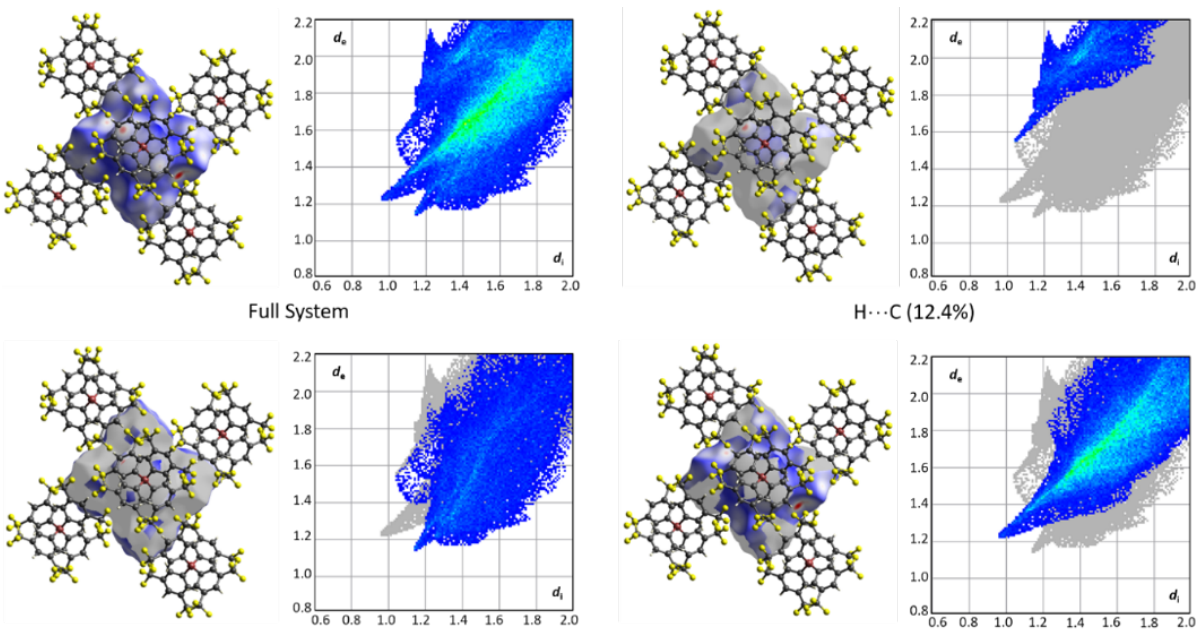

$H \cdots H(24.2 \%)$

$+\cdot+6(63.4 \%)$

Figure S72: Hirshfeld Surfaces showing normalised distance and associated

Figure S71: Hirshfeld Surfaces showing normalised distance and associated fingerprint plots in [1-propane][BAr ${ }_{4}$ ] (fully optimised). Percentage of each fingerprint plots in [1-propane][BAr${ }_{4}$ ] (partially optimised). Percentage of type of contact is shown in brackets.

each type of contact is shown in brackets. 


\section{S.5.2. [1-pentane][BAr $\left.{ }_{4}\right]$}

Note: The structure of $\left[\mathrm{Rh}\left(\mathrm{Cy}_{2} \mathrm{PCH}_{2} \mathrm{CH}_{2} \mathrm{PCy}\right)\left(\mathrm{C}_{5} \mathrm{H}_{12}\right)\right]\left[\mathrm{BAr}_{4}\right]$, [1-pentane][BAr$\left.{ }_{4}\right]$, has been previous reported. ${ }^{\mathrm{S} 1}$ We now report results using the protocol adopted in this paper for consistency.

\section{[1-pentane][BAr $\left.{ }_{4}\right]$ (Partially Optimised)}

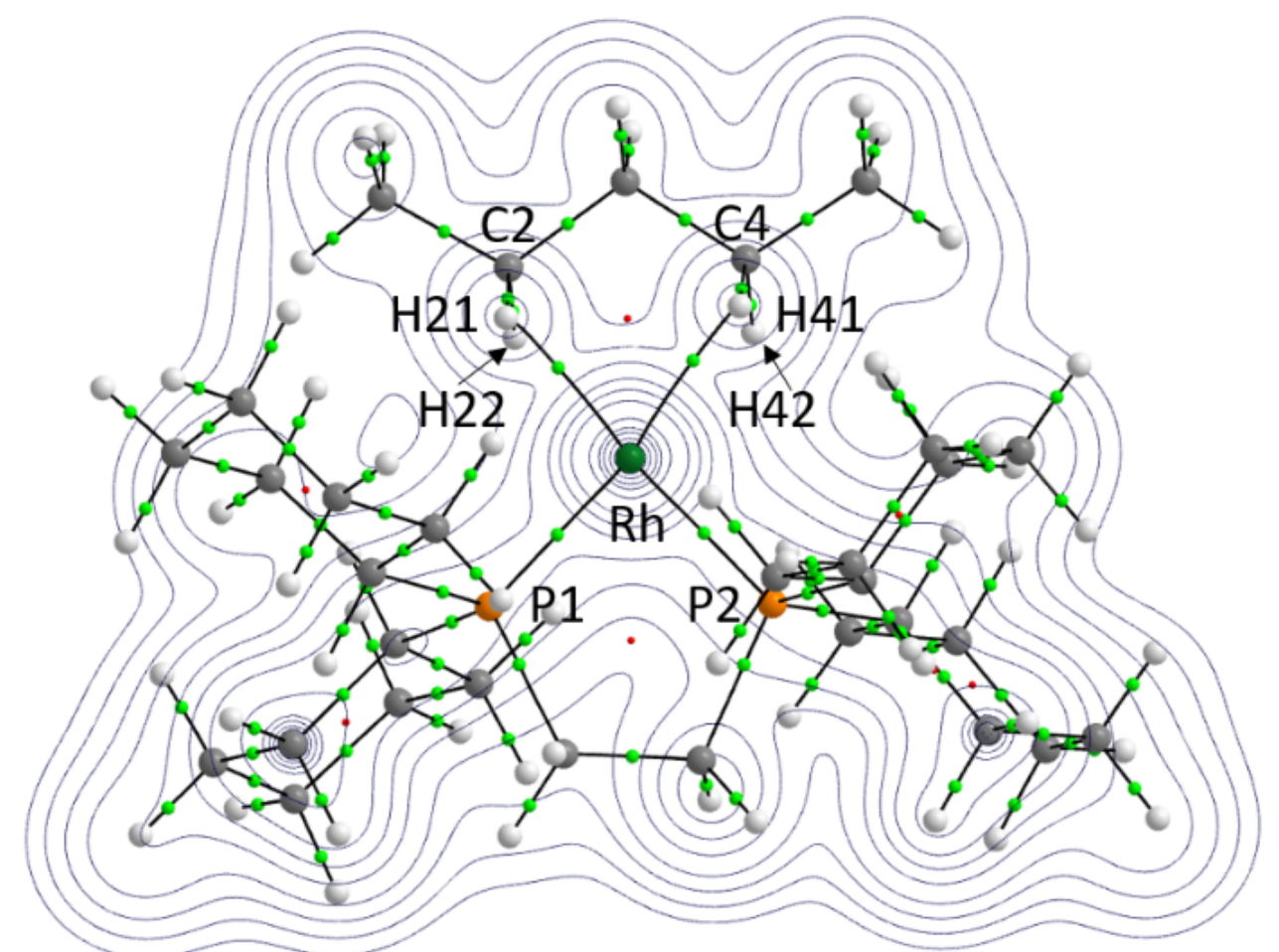

Table S5: QTAIM BCP data and bond lengths for key bonds.

\begin{tabular}{|c|c|c|c|c|}
\hline & Interatomic & & & \\
\hline $\begin{array}{l}\text { Bond } \\
\text { Path }\end{array}$ & $\begin{array}{c}\text { Distance } \\
(\AA)\end{array}$ & $\begin{array}{c}\rho \\
\left(\text { e bohr }^{-3}\right)\end{array}$ & $\begin{array}{c}\nabla^{2} \rho \\
\left(e \text { bohr } r^{-5}\right)\end{array}$ & $H(r)$ \\
\hline $\mathrm{C} 2-\mathrm{H} 21$ & 1.140 & 0.2488 & -0.6912 & -0.2212 \\
\hline $\mathrm{H} 21-\mathrm{Rh}$ & 1.998 & 0.0473 & 0.1770 & -0.0052 \\
\hline C2-Rh & 2.522 & & No BCP & \\
\hline Rh-P1 & 2.197 & 0.1212 & 0.0668 & -0.1388 \\
\hline $\mathrm{C} 4-\mathrm{H} 41$ & 1.136 & 0.2472 & -0.7156 & -0.2299 \\
\hline $\mathrm{H} 41-\mathrm{Rh}$ & 2.029 & 0.0458 & 0.1709 & -0.0043 \\
\hline C4-Rh & 2.513 & & No BCP & \\
\hline Rh-P2 & 2.196 & 0.1216 & 0.0665 & -0.1392 \\
\hline
\end{tabular}

Figure S73: QTAIM molecular graph of [1-pentane][BAr $\left.{ }_{4}\right]$ (partially optimised) with electron density contours in the $\mathrm{H} 21-\mathrm{Rh}-\mathrm{H} 41$ plane. 


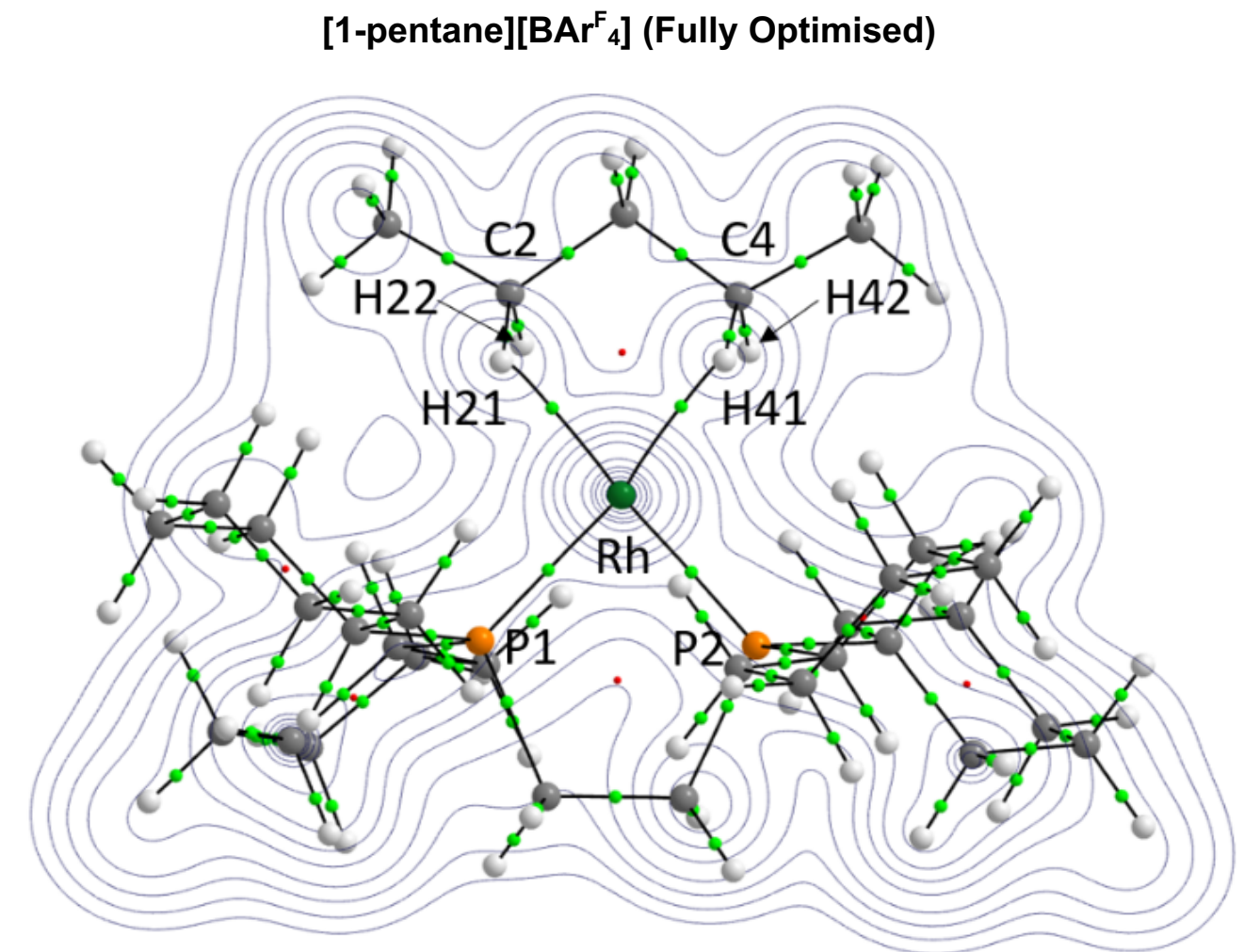

Table S6: QTAIM BCP data and bond lengths for key bonds.

\begin{tabular}{ccccc}
\hline $\begin{array}{c}\text { Bond } \\
\text { Path }\end{array}$ & $\begin{array}{c}\text { Interatomic } \\
\text { Distance } \\
(\mathbf{A})\end{array}$ & $\begin{array}{c}\boldsymbol{\rho} \\
\left(\mathbf{e} \mathbf{b o h h}^{-3}\right)\end{array}$ & $\begin{array}{c}\boldsymbol{\nabla}^{2} \boldsymbol{\rho} \\
(\mathbf{e} \text { bohr }\end{array}$ & $\mathbf{H})$ \\
\hline C2 -H21 & 1.141 & 0.2428 & -0.6843 & -0.2195 \\
H21-Rh & 1.998 & 0.0472 & 0.1742 & -0.0054 \\
C2-Rh & 2.534 & & No BCP & \\
Rh-P1 & 2.207 & 0.1191 & 0.0722 & -0.0591 \\
C4-H41 & 1.137 & 0.2465 & -0.7103 & -0.2256 \\
H41-Rh & 2.020 & 0.0468 & 0.1721 & -0.0048 \\
C4-Rh & 2.509 & & No BCP & \\
Rh-P2 & 2.207 & 0.1189 & 0.0731 & -0.0588 \\
\hline
\end{tabular}

Figure S74: QTAIM molecular graph of [1-pentane][BAr ${ }_{4}$ ] (fully optimised) with electron density contours in the H21-Rh-H41 plane. 

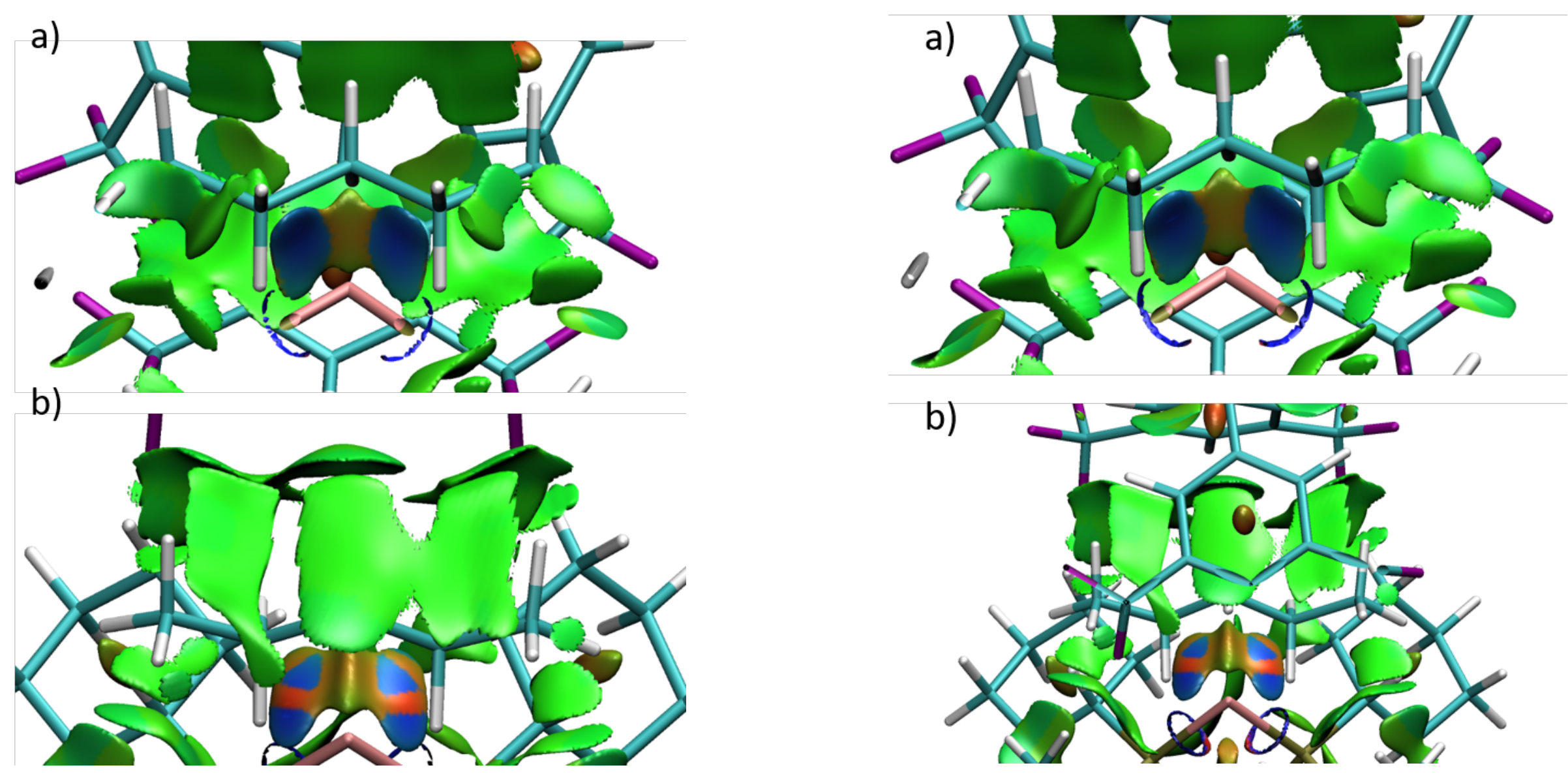

Figure S75: a) $\mathrm{NCl}$ Plot of [1-pentane][BAr${ }_{4}$ ] (partially optimised) with Figure S76: a) $\mathrm{NCl}$ Plot of [1-pentane][BAr ${ }_{4}$ ] (fully optimised) with isosurfaces generated for $s=0.3$ au and $-0.07<\rho<0.07$ au. View from Rh isosurfaces generated for $s=0.3$ au and $-0.07<\rho<0.07$ au. View from Rh looking towards the alkane. b) View from above the alkane looking towards looking towards the alkane. b) View from above the alkane looking towards Rh.

$\mathrm{Rh}$. 
Table S7: NBO data from [1-pentane][BAr ${ }_{4}$ ] (partially optimised) showing the donating and accepting orbitals occupation and the $2^{\text {nd }}$ order perturbation theory interaction energy.

\begin{tabular}{|c|c|c|c|c|}
\hline $\begin{array}{c}\text { Donating } \\
\text { Orbital }\end{array}$ & Occupation & $\begin{array}{l}\text { Accepting } \\
\text { Orbital }\end{array}$ & Occupation & $\begin{array}{c}\text { Energy } \\
\text { (kcal/mol) }\end{array}$ \\
\hline \multicolumn{5}{|c|}{$\mathrm{C} 2-\mathrm{H} 21$} \\
\hline$\sigma(\mathrm{C}-\mathrm{H})$ & 1.92 & $\sigma^{*}\left(\mathrm{Rh}-\mathrm{P}_{\text {trans }}\right)$ & 0.25 & 11.63 \\
\hline$\sigma(\mathrm{C}-\mathrm{H})$ & 1.92 & $\sigma^{*}\left(\mathrm{Rh}-\mathrm{P}_{\mathrm{Cis}}\right)$ & 0.26 & 0.14 \\
\hline $\mathrm{LP}(\mathrm{Rh})$ & 1.96 & $\sigma^{*}(\mathrm{C}-\mathrm{H})$ & 0.04 & 0.98 \\
\hline $\mathrm{LP}(\mathrm{Rh})$ & 1.94 & $\sigma^{*}(\mathrm{C}-\mathrm{H})$ & 0.04 & 1.66 \\
\hline $\mathrm{LP}(\mathrm{Rh})$ & 1.89 & $\sigma^{*}(\mathrm{C}-\mathrm{H})$ & 0.04 & 2.52 \\
\hline$\sigma\left(\mathrm{Rh}-\mathrm{P}_{\text {cis }}\right)$ & 1.83 & $\sigma^{*}(\mathrm{C}-\mathrm{H})$ & 0.04 & 1.96 \\
\hline$\sigma\left(\mathrm{Rh}-\mathrm{P}_{\text {trans }}\right)$ & 1.83 & $\sigma^{*}(\mathrm{C}-\mathrm{H})$ & 0.04 & 1.00 \\
\hline \multicolumn{5}{|c|}{$\mathrm{C} 2-\mathrm{H} 22$} \\
\hline$\sigma(\mathrm{C}-\mathrm{H})$ & 1.96 & $\sigma^{*}\left(\mathrm{Rh}-\mathrm{P}_{\text {trans }}\right)$ & 0.25 & 3.61 \\
\hline $\mathrm{LP}(\mathrm{Rh})$ & 1.96 & $\sigma^{*}(\mathrm{C}-\mathrm{H})$ & 0.25 & 0.58 \\
\hline $\mathrm{LP}(\mathrm{Rh})$ & 1.94 & $\sigma^{*}(\mathrm{C}-\mathrm{H})$ & 0.25 & 0.55 \\
\hline $\mathrm{LP}(\mathrm{Rh})$ & 1.90 & $\sigma^{*}(\mathrm{C}-\mathrm{H})$ & 0.25 & 0.26 \\
\hline $\mathrm{LP}(\mathrm{Rh})$ & 1.89 & $\sigma^{*}(\mathrm{C}-\mathrm{H})$ & 0.25 & 0.14 \\
\hline$\sigma\left(\mathrm{Rh}-\mathrm{P}_{\text {cis }}\right)$ & 1.83 & $\sigma^{*}(\mathrm{C}-\mathrm{H})$ & 0.25 & 0.54 \\
\hline$\sigma\left(\mathrm{Rh}-\mathrm{P}_{\text {trans }}\right)$ & 1.83 & $\sigma^{*}(\mathrm{C}-\mathrm{H})$ & 0.25 & 0.33 \\
\hline \multicolumn{5}{|c|}{$\mathrm{C} 4-\mathrm{H} 41$} \\
\hline$\sigma(\mathrm{C}-\mathrm{H})$ & 1.91 & $\sigma^{*}\left(\mathrm{Rh}-\mathrm{P}_{\text {trans }}\right)$ & 0.26 & 13.06 \\
\hline$\sigma(\mathrm{C}-\mathrm{H})$ & 1.91 & $\sigma^{*}\left(\mathrm{Rh}-\mathrm{P}_{\mathrm{Cis}}\right)$ & 0.25 & 0.06 \\
\hline
\end{tabular}

\begin{tabular}{ccccc} 
LP $(\mathrm{Rh})$ & 1.96 & $\sigma^{*}(\mathrm{C}-\mathrm{H})$ & 0.04 & 0.17 \\
LP $(\mathrm{Rh})$ & 1.94 & $\sigma^{*}(\mathrm{C}-\mathrm{H})$ & 0.04 & 2.21 \\
LP $(\mathrm{Rh})$ & 1.89 & $\sigma^{*}(\mathrm{C}-\mathrm{H})$ & 0.04 & 2.86 \\
$\sigma\left(\mathrm{Rh}-\mathrm{P}_{\text {cis }}\right)$ & 1.83 & $\sigma^{*}(\mathrm{C}-\mathrm{H})$ & 0.04 & 2.27 \\
$\sigma\left(\mathrm{Rh}-\mathrm{P}_{\text {trans }}\right)$ & 1.83 & $\sigma^{*}(\mathrm{C}-\mathrm{H})$ & 0.04 & 0.98 \\
\hline \multicolumn{5}{c}{$\mathrm{C} 4-\mathrm{H} 42$} \\
\hline$\sigma(\mathrm{C}-\mathrm{H})$ & 1.96 & $\sigma^{*}\left(\mathrm{Rh}-\mathrm{P}_{\text {trans }}\right)$ & 0.26 \\
LP $(\mathrm{Rh})$ & 1.96 & $\sigma^{*}(\mathrm{C}-\mathrm{H})$ & 0.22 & 0.84 \\
LP $(\mathrm{Rh})$ & 1.94 & $\sigma^{*}(\mathrm{C}-\mathrm{H})$ & 0.22 & 0.17 \\
LP $(\mathrm{Rh})$ & 1.90 & $\sigma^{*}(\mathrm{C}-\mathrm{H})$ & 0.22 & 0.15 \\
LP $(\mathrm{Rh})$ & 1.89 & $\sigma^{*}(\mathrm{C}-\mathrm{H})$ & 0.22 & 0.09 \\
$\sigma\left(\mathrm{Rh}-\mathrm{P}_{\text {cis }}\right)$ & 1.83 & $\sigma^{*}(\mathrm{C}-\mathrm{H})$ & 0.22 & 0.45 \\
$\sigma\left(\mathrm{Rh}-\mathrm{P}_{\text {trans }}\right)$ & 1.83 & $\sigma^{*}(\mathrm{C}-\mathrm{H})$ & 0.22 & 0.30 \\
\hline
\end{tabular}


Table S8: NBO data from [1-pentane][BAr ${ }_{4}$ ] (fully optimised) showing the donating and accepting orbitals occupation and the $2^{\text {nd }}$ order perturbation theory interaction energy.

\begin{tabular}{|c|c|c|c|c|}
\hline $\begin{array}{c}\text { Donating } \\
\text { Orbital }\end{array}$ & Occupation & $\begin{array}{c}\text { Accepting } \\
\text { Orbital }\end{array}$ & Occupation & $\begin{array}{c}\text { Energy } \\
\text { (kcal/mol) }\end{array}$ \\
\hline \multicolumn{5}{|c|}{$\mathrm{C} 2-\mathrm{H} 21$} \\
\hline$\sigma(\mathrm{C}-\mathrm{H})$ & 1.92 & $\sigma^{*}\left(\mathrm{Rh}-\mathrm{P}_{\text {trans }}\right)$ & 0.26 & 12.01 \\
\hline$\sigma(\mathrm{C}-\mathrm{H})$ & 1.92 & $\sigma^{*}\left(\mathrm{Rh}-\mathrm{P}_{\mathrm{Cis}}\right)$ & 0.25 & 0.17 \\
\hline LP (Rh) & 1.96 & $\sigma^{*}(\mathrm{C}-\mathrm{H})$ & 0.04 & 1.12 \\
\hline LP (Rh) & 1.94 & $\sigma^{*}(\mathrm{C}-\mathrm{H})$ & 0.04 & 1.56 \\
\hline $\mathrm{LP}(\mathrm{Rh})$ & 1.90 & $\sigma^{*}(\mathrm{C}-\mathrm{H})$ & 0.04 & 0.12 \\
\hline $\mathrm{LP}(\mathrm{Rh})$ & 1.89 & $\sigma^{*}(\mathrm{C}-\mathrm{H})$ & 0.04 & 2.58 \\
\hline$\sigma\left(\mathrm{Rh}-\mathrm{P}_{\text {cis }}\right)$ & 1.83 & $\sigma^{*}(\mathrm{C}-\mathrm{H})$ & 0.04 & 1.96 \\
\hline$\sigma\left(\mathrm{Rh}-P_{\text {trans }}\right)$ & 1.83 & $\sigma^{*}(\mathrm{C}-\mathrm{H})$ & 0.04 & 1.02 \\
\hline \multicolumn{5}{|c|}{$\mathrm{C} 2-\mathrm{H} 22$} \\
\hline$\sigma(\mathrm{C}-\mathrm{H})$ & 1.95 & $\sigma^{*}\left(\mathrm{Rh}-\mathrm{P}_{\text {trans }}\right)$ & 0.25 & 4.07 \\
\hline $\mathrm{LP}(\mathrm{Rh})$ & 1.96 & $\sigma^{*}(\mathrm{C}-\mathrm{H})$ & 0.02 & 0.61 \\
\hline $\mathrm{LP}(\mathrm{Rh})$ & 1.94 & $\sigma^{*}(\mathrm{C}-\mathrm{H})$ & 0.02 & 0.64 \\
\hline $\mathrm{LP}(\mathrm{Rh})$ & 1.90 & $\sigma^{*}(\mathrm{C}-\mathrm{H})$ & 0.02 & 0.35 \\
\hline LP (Rh) & 1.89 & $\sigma^{*}(\mathrm{C}-\mathrm{H})$ & 0.02 & 0.11 \\
\hline$\sigma\left(\mathrm{Rh}-\mathrm{P}_{\text {cis }}\right)$ & 1.83 & $\sigma^{*}(\mathrm{C}-\mathrm{H})$ & 0.02 & 0.61 \\
\hline$\sigma\left(\mathrm{Rh}-\mathrm{P}_{\text {trans }}\right)$ & 1.83 & $\sigma^{*}(\mathrm{C}-\mathrm{H})$ & 0.02 & 0.36 \\
\hline \multicolumn{5}{|c|}{$\mathrm{C} 4-\mathrm{H} 41$} \\
\hline$\sigma(\mathrm{C}-\mathrm{H})$ & 1.91 & $\sigma^{*}\left(\mathrm{Rh}-\mathrm{P}_{\text {trans }}\right)$ & 0.26 & 13.25 \\
\hline$\sigma(\mathrm{C}-\mathrm{H})$ & 1.91 & $\sigma^{*}\left(\mathrm{Rh}-\mathrm{P}_{\mathrm{Cis}}\right)$ & 0.25 & 0.07 \\
\hline
\end{tabular}

\begin{tabular}{|c|c|c|c|c|}
\hline LP (Rh) & 1.96 & $\sigma^{*}(\mathrm{C}-\mathrm{H})$ & 0.04 & 0.12 \\
\hline LP (Rh) & 1.94 & $\sigma^{*}(\mathrm{C}-\mathrm{H})$ & 0.04 & 2.28 \\
\hline LP (Rh) & 1.89 & $\sigma^{*}(\mathrm{C}-\mathrm{H})$ & 0.04 & 2.75 \\
\hline$\sigma\left(\mathrm{Rh}-\mathrm{P}_{\text {cis }}\right)$ & 1.83 & $\sigma^{*}(\mathrm{C}-\mathrm{H})$ & 0.04 & 2.28 \\
\hline$\sigma\left(\mathrm{Rh}-\mathrm{P}_{\text {trans }}\right)$ & 1.83 & $\sigma^{*}(\mathrm{C}-\mathrm{H})$ & 0.04 & 1.01 \\
\hline \multicolumn{5}{|c|}{$\mathrm{C} 4-\mathrm{H} 42$} \\
\hline$\sigma(\mathrm{C}-\mathrm{H})$ & 1.96 & $\sigma^{*}\left(\mathrm{Rh}-\mathrm{P}_{\text {trans }}\right)$ & 0.26 & 2.57 \\
\hline LP (Rh) & 1.96 & $\sigma^{*}(\mathrm{C}-\mathrm{H})$ & 0.02 & 0.89 \\
\hline LP (Rh) & 1.94 & $\sigma^{*}(\mathrm{C}-\mathrm{H})$ & 0.02 & 0.15 \\
\hline LP (Rh) & 1.90 & $\sigma^{*}(\mathrm{C}-\mathrm{H})$ & 0.02 & 0.18 \\
\hline $\mathrm{LP}(\mathrm{Rh})$ & 1.89 & $\sigma^{*}(\mathrm{C}-\mathrm{H})$ & 0.02 & 0.06 \\
\hline$\sigma\left(\mathrm{Rh}-\mathrm{P}_{\text {cis }}\right)$ & 1.83 & $\sigma^{*}(\mathrm{C}-\mathrm{H})$ & 0.02 & 0.47 \\
\hline$\sigma\left(\mathrm{Rh}-\mathrm{P}_{\text {trans }}\right)$ & 1.83 & $\sigma^{*}(\mathrm{C}-\mathrm{H})$ & 0.02 & 0.32 \\
\hline
\end{tabular}



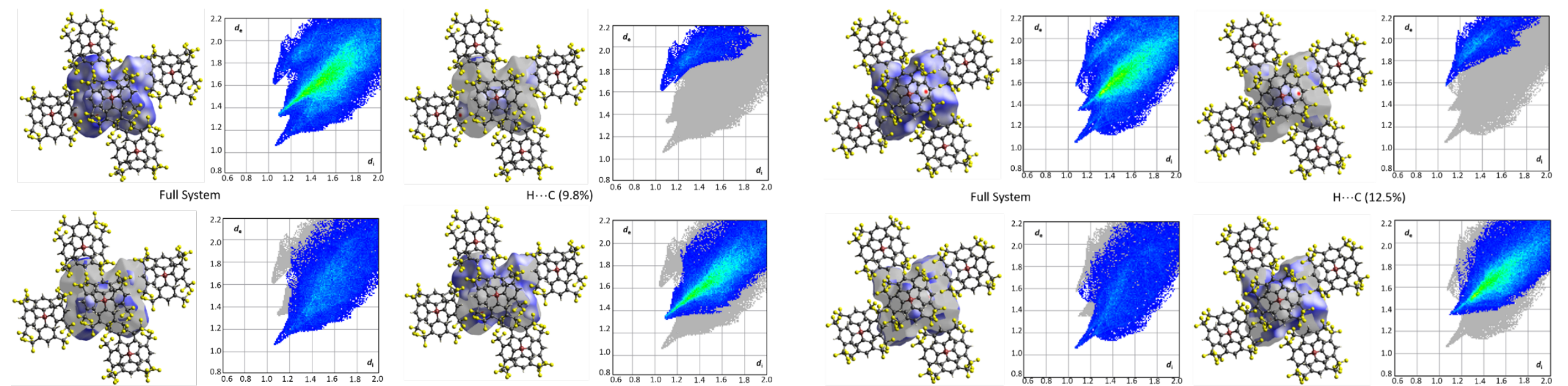

H ...F (56.6\%)

H...H (24.9\%)

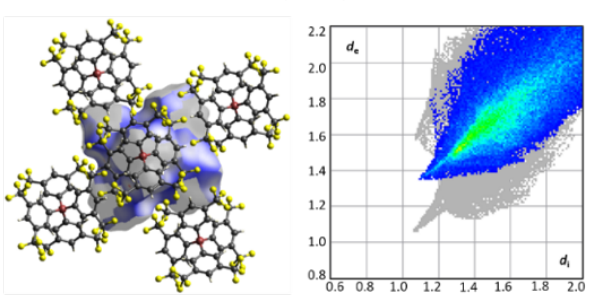

H...F (62.6\%)

Figure S77: Hirshfeld Surfaces of [1-pentane][BAr ${ }_{4}$ ] (partially optimised) Figure S78: Hirshfeld Surfaces of [1-pentane][BAr ${ }_{4}$ ] (fully optimised) showing normalised distance and associated fingerprint plots. Percentage of showing normalised distance and associated fingerprint plots. Percentage of each type of contact is shown in brackets. each type of contact is shown in brackets. 


\section{S.5.3. [1-(2-methylbutane)][BAr $\left.{ }_{4}{ }_{4}\right]$}

\section{[1-(2-methylbutane)][BAr $\left.{ }_{4}\right]$ (Partially Optimised)}

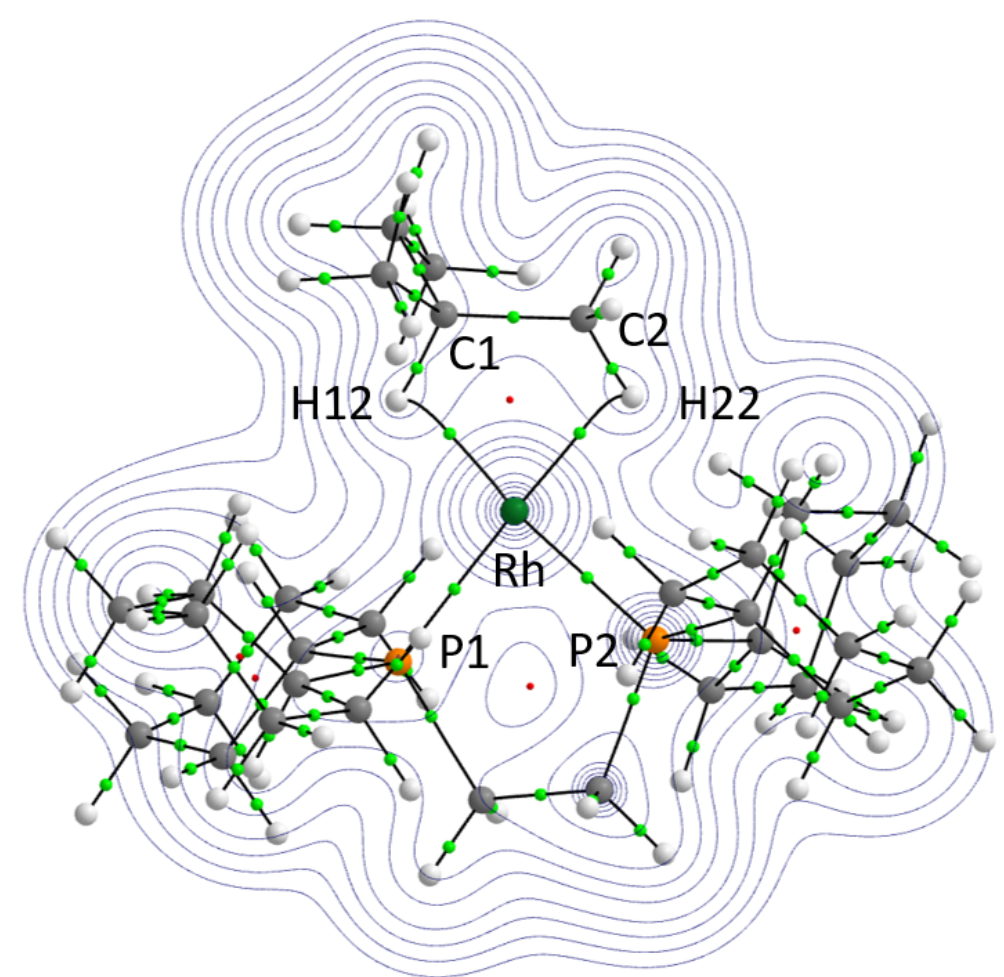

Figure S79: QTAIM molecular graph of [1-(2-methylbutane)][BAr ${ }_{4}$ ] (partially optimised) with electron density contours in the H12-Rh-H22 plane.
Table S9: QTAIM BCP data and bond lengths for key bonds.

\begin{tabular}{|c|c|c|c|c|}
\hline & Interatomic & & & \\
\hline $\begin{array}{l}\text { Bond } \\
\text { Path }\end{array}$ & $\begin{array}{c}\text { Distance } \\
\text { (A) }\end{array}$ & $\begin{array}{c}\rho \\
\left(e^{-} b o h r^{-3}\right)\end{array}$ & $\begin{array}{c}\nabla^{2} \rho \\
\left(e b^{-5}\right)\end{array}$ & $H(r)$ \\
\hline $\mathrm{C} 1-\mathrm{H} 12$ & 1.160 & 0.2312 & -0.5990 & -0.1992 \\
\hline $\mathrm{H} 12-\mathrm{Rh}$ & 1.846 & 0.0646 & 0.2634 & -0.0100 \\
\hline C1-Rh & 2.390 & & No BCP & \\
\hline Rh-P1 & 2.187 & 0.1237 & 0.0666 & -0.0643 \\
\hline $\mathrm{C} 2-\mathrm{H} 22$ & 1.145 & 0.2385 & -0.6560 & -0.2133 \\
\hline $\mathrm{H} 22-\mathrm{Rh}$ & 1.878 & 0.0622 & 0.2580 & -0.0085 \\
\hline C2-Rh & 2.349 & & No BCP & \\
\hline Rh-P2 & 2.185 & 0.1237 & 0.0699 & -0.0643 \\
\hline
\end{tabular}




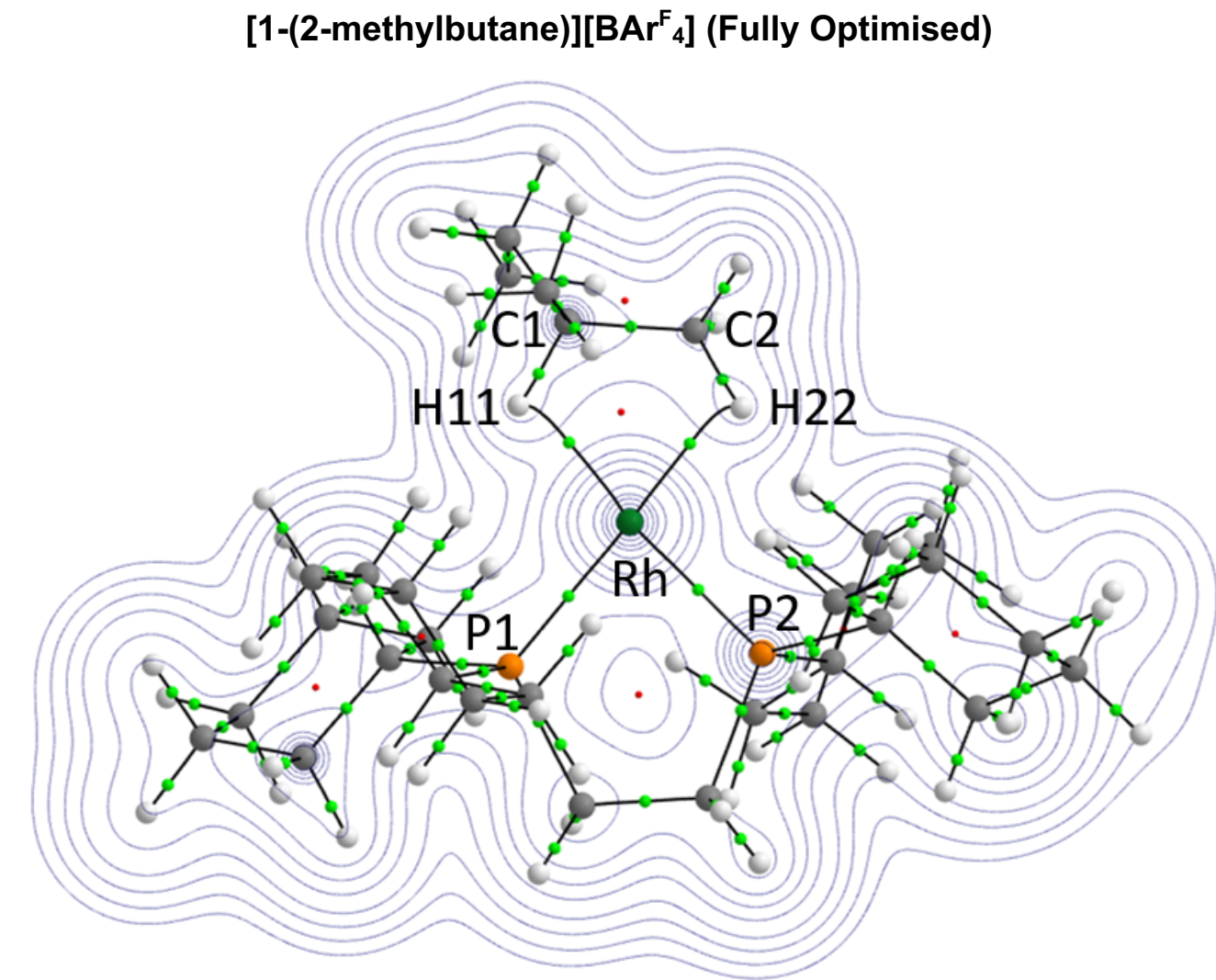

Table S10: QTAIM BCP data and bond lengths for key bonds.

\begin{tabular}{|c|c|c|c|c|}
\hline \multirow[b]{2}{*}{$\begin{array}{l}\text { Bond } \\
\text { Path }\end{array}$} & \multicolumn{4}{|c|}{ Interatomic } \\
\hline & $\begin{array}{c}\text { Distance } \\
(\AA)\end{array}$ & $\begin{array}{c}\rho \\
\left(e b^{-3}\right)\end{array}$ & $\begin{array}{c}\nabla^{2} \rho \\
\left(\text { e bohr } r^{-5}\right)\end{array}$ & $H(r)$ \\
\hline $\mathrm{C} 1-\mathrm{H} 11$ & 1.161 & 0.2304 & -0.5978 & -0.1979 \\
\hline $\mathrm{H} 11-\mathrm{Rh}$ & 1.899 & 0.0568 & 0.2245 & -0.0077 \\
\hline C1-Rh & 2.475 & & No BCP & \\
\hline Rh-P1 & 2.211 & 0.1182 & 0.0782 & -0.0579 \\
\hline $\mathrm{C} 2-\mathrm{H} 22$ & 1.150 & 0.2352 & -0.6331 & -0.2073 \\
\hline $\mathrm{H} 22-\mathrm{Rh}$ & 1.909 & 0.0579 & 0.2413 & -0.0068 \\
\hline $\mathrm{C} 2-\mathrm{Rh}$ & 2.381 & & No $B C P$ & \\
\hline Rh-P2 & 2.200 & 0.1207 & 0.0708 & -0.0608 \\
\hline
\end{tabular}

Figure S80: QTAIM molecular graph of [1-(2-methylbutane)][BAr ${ }_{4}$ ] (fully optimised) with electron density contours in the $\mathrm{H} 11-\mathrm{Rh}-\mathrm{H} 22$ plane. 

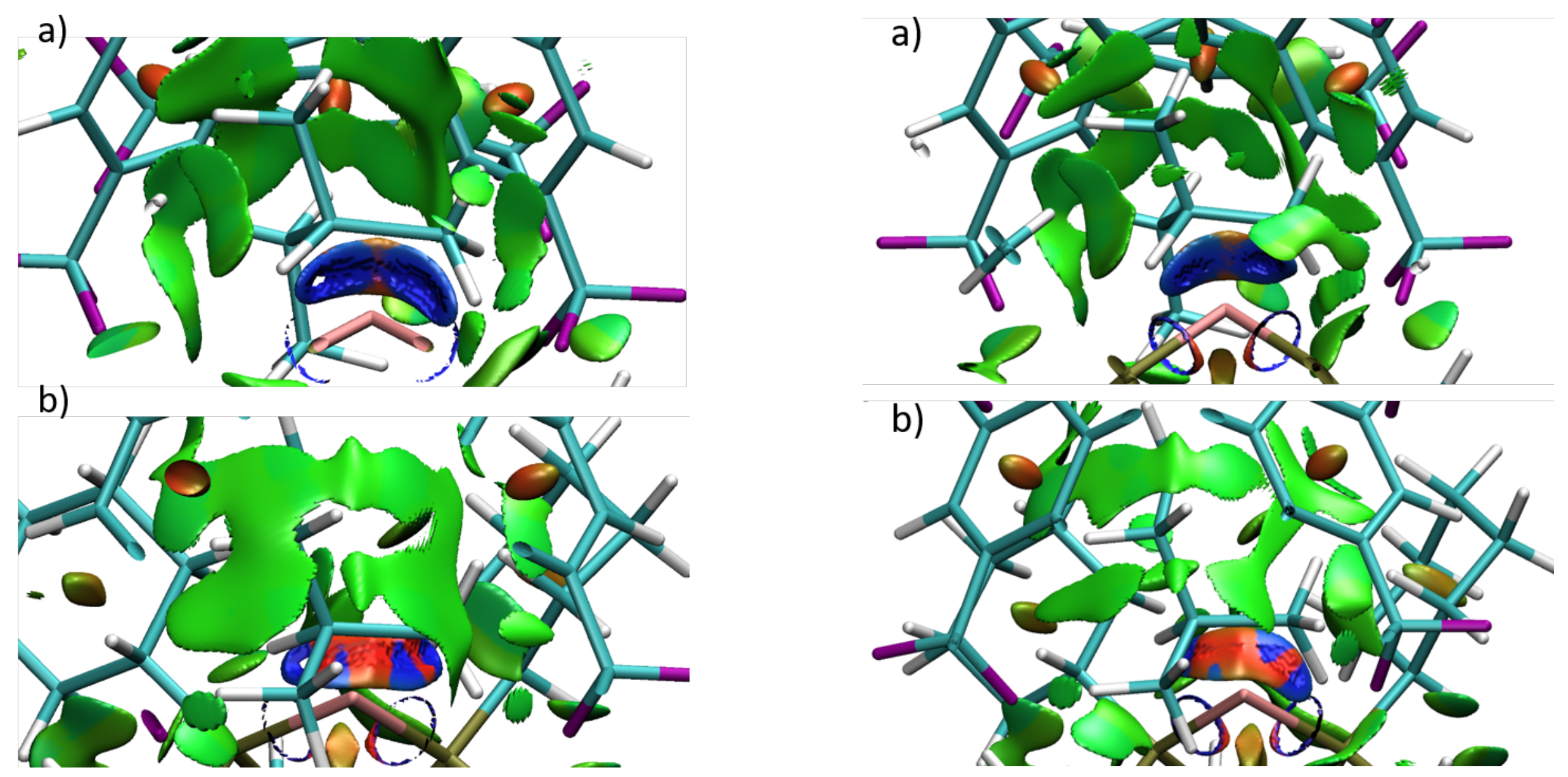

Figure S81: a) NCI Plot of [1-(2-methylbutane)][BAr ${ }_{4}$ ] (partially optimised) Figure S82: a) NCl Plot of [1-(2-methylbutane)][BAr ${ }_{4}$ ] (fully optimised) with with isosurfaces generated for $s=0.3$ au and $-0.07<\rho<0.07$ au. View from isosurfaces generated for $s=0.3$ au and $-0.07<\rho<0.07$ au. View from Rh $\mathrm{Rh}$ looking towards the alkane. b) View from above the alkane looking looking towards the alkane. b) View from above the alkane looking towards towards Rh. 
Table S11: NBO data from [1-(2-methylbutane)][BAr ${ }_{4}$ ] (partially optimised) Table S12: NBO data from [1-(2-methylbutane)][BAr $\left.{ }_{4}\right]$ (fully optimised) showing the donating and accepting orbitals occupation and the $2^{\text {nd }}$ order showing the donating and accepting orbitals occupation and the $2^{\text {nd }}$ order perturbation theory interaction energy.

\begin{tabular}{|c|c|c|c|c|}
\hline $\begin{array}{c}\text { Donating } \\
\text { Orbital }\end{array}$ & Occupation & $\begin{array}{c}\text { Accepting } \\
\text { Orbital }\end{array}$ & Occupation & $\begin{array}{c}\text { Energy } \\
\text { (kcal/mol) }\end{array}$ \\
\hline \multicolumn{5}{|c|}{$\mathrm{C} 1-\mathrm{H} 11$} \\
\hline$\sigma(\mathrm{C}-\mathrm{H})$ & 1.86 & $\sigma^{*}\left(\mathrm{Rh}-\mathrm{P}_{\text {trans }}\right)$ & 0.29 & 22.71 \\
\hline$\sigma(\mathrm{C}-\mathrm{H})$ & 1.86 & $\sigma^{*}\left(\mathrm{Rh}-\mathrm{P}_{\mathrm{CIS}}\right)$ & 0.28 & 0.30 \\
\hline LP (Rh) & 1.97 & $\sigma^{*}(\mathrm{C}-\mathrm{H})$ & 0.05 & 0.07 \\
\hline LP (Rh) & 1.95 & $\sigma^{*}(\mathrm{C}-\mathrm{H})$ & 0.05 & 0.18 \\
\hline LP (Rh) & 1.87 & $\sigma^{*}(\mathrm{C}-\mathrm{H})$ & 0.05 & 3.95 \\
\hline$\sigma\left(\mathrm{Rh}-\mathrm{P}_{\text {cis }}\right)$ & 1.83 & $\sigma^{*}(\mathrm{C}-\mathrm{H})$ & 0.05 & 4.14 \\
\hline $\begin{array}{c}\sigma(\mathrm{Rh}- \\
\left.\mathrm{P}_{\text {trans }}\right)\end{array}$ & 1.83 & $\sigma^{*}(\mathrm{C}-\mathrm{H})$ & 0.05 & 1.50 \\
\hline \multicolumn{5}{|c|}{$\mathrm{C} 2-\mathrm{H} 22$} \\
\hline$\sigma(\mathrm{C}-\mathrm{H})$ & 1.88 & $\sigma^{*}\left(\mathrm{Rh}-\mathrm{P}_{\text {trans }}\right)$ & 0.28 & 24.74 \\
\hline$\sigma(\mathrm{C}-\mathrm{H})$ & 1.88 & $\sigma^{*}\left(\mathrm{Rh}-\mathrm{P}_{\mathrm{CIS}}\right)$ & 0.29 & 0.28 \\
\hline LP (Rh) & 1.95 & $\sigma^{*}(\mathrm{C}-\mathrm{H})$ & 0.03 & 1.07 \\
\hline LP (Rh) & 1.91 & $\sigma^{*}(\mathrm{C}-\mathrm{H})$ & 0.03 & 0.24 \\
\hline LP (Rh) & 1.87 & $\sigma^{*}(\mathrm{C}-\mathrm{H})$ & 0.03 & 4.88 \\
\hline$\sigma\left(\mathrm{Rh}-\mathrm{P}_{\text {cis }}\right)$ & 1.83 & $\sigma^{*}(\mathrm{C}-\mathrm{H})$ & 0.03 & 3.34 \\
\hline$\sigma\left(R h-P_{\text {trans }}\right)$ & 1.83 & $\sigma^{*}(\mathrm{C}-\mathrm{H})$ & 0.03 & 0.76 \\
\hline
\end{tabular}
perturbation theory interaction energy.

\begin{tabular}{ccccc}
\hline $\begin{array}{c}\text { Donating } \\
\text { Orbital }\end{array}$ & Occupation & $\begin{array}{c}\text { Accepting } \\
\text { Orbital }\end{array}$ & Occupation & $\begin{array}{c}\text { Energy } \\
\text { (kcal/mol) }\end{array}$ \\
\hline$\sigma(\mathrm{C}-\mathrm{H})$ & 1.86 & $\sigma^{*}\left(\mathrm{Rh}-\mathrm{P}_{\text {trans }}\right)$ & 0.28 & 19.80 \\
$\sigma(\mathrm{C}-\mathrm{H})$ & 1.86 & $\sigma^{*}\left(\mathrm{Rh}-\mathrm{P}_{\text {cis }}\right)$ & 0.27 & 0.25 \\
LP $(\mathrm{Rh})$ & 1.97 & $\sigma^{*}(\mathrm{C}-\mathrm{H})$ & 0.05 & 0.09 \\
LP $(\mathrm{Rh})$ & 1.95 & $\sigma^{*}(\mathrm{C}-\mathrm{H})$ & 0.05 & 0.12 \\
LP $(\mathrm{Rh})$ & 1.91 & $\sigma^{*}(\mathrm{C}-\mathrm{H})$ & 0.05 & 0.44 \\
LP $(\mathrm{Rh})$ & 1.88 & $\sigma^{*}(\mathrm{C}-\mathrm{H})$ & 0.05 & 2.65 \\
$\sigma\left(\mathrm{Rh}-\mathrm{P}_{\text {cis }}\right)$ & 1.83 & $\sigma^{*}(\mathrm{C}-\mathrm{H})$ & 0.05 & 3.67 \\
$\sigma\left(\mathrm{Rh}-\mathrm{P}_{\text {trans }}\right)$ & 1.83 & $\sigma^{*}(\mathrm{C}-\mathrm{H})$ & 0.05 & 1.79 \\
\hline & & $\mathrm{C} 2-\mathrm{H} 22$ & & \\
\hline$\sigma(\mathrm{C}-\mathrm{H})$ & 1.88 & $\sigma^{*}\left(\mathrm{Rh}-\mathrm{P}_{\text {trans }}\right)$ & 0.27 & 21.93 \\
$\sigma(\mathrm{C}-\mathrm{H})$ & 1.88 & $\sigma^{*}\left(\mathrm{Rh}-\mathrm{P}_{\text {cIS }}\right)$ & 0.28 & 0.24 \\
LP $(\mathrm{Rh})$ & 1.95 & $\sigma^{*}(\mathrm{C}-\mathrm{H})$ & 0.03 & 1.22 \\
LP $(\mathrm{Rh})$ & 1.91 & $\sigma^{*}(\mathrm{C}-\mathrm{H})$ & 0.03 & 1.01 \\
LP $(\mathrm{Rh})$ & 1.88 & $\sigma^{*}(\mathrm{C}-\mathrm{H})$ & 0.03 & 3.42 \\
$\sigma\left(\mathrm{Rh}-\mathrm{P}_{\text {cis }}\right)$ & 1.83 & $\sigma^{*}(\mathrm{C}-\mathrm{H})$ & 0.03 & 2.45 \\
$\sigma\left(\mathrm{Rh}-\mathrm{P}_{\text {trans }}\right)$ & 1.83 & $\sigma^{*}(\mathrm{C}-\mathrm{H})$ & 0.03 & 0.79 \\
\hline
\end{tabular}




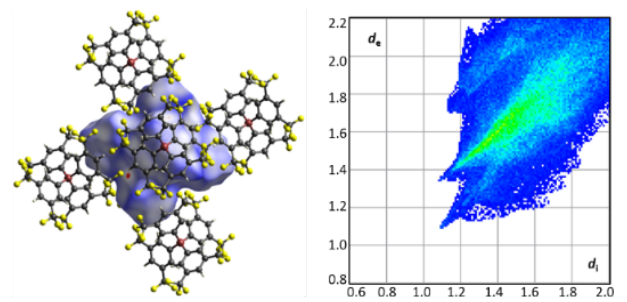

Full System

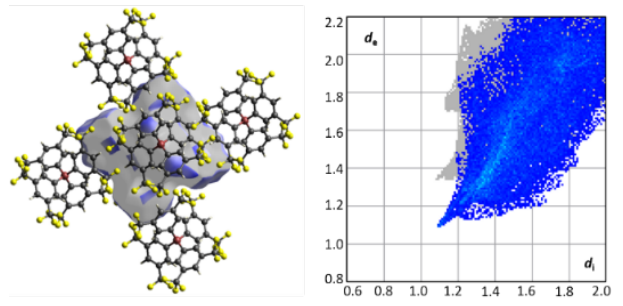

$\mathrm{H} \cdots \mathrm{H}(25.2 \%)$

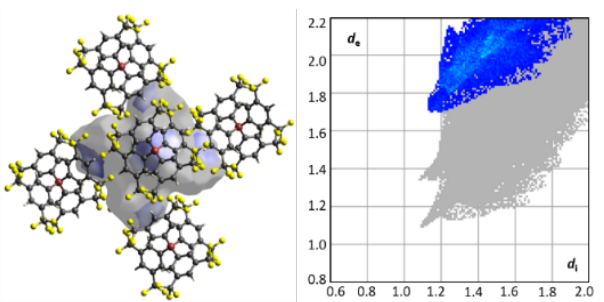

H...C (12.2\%)

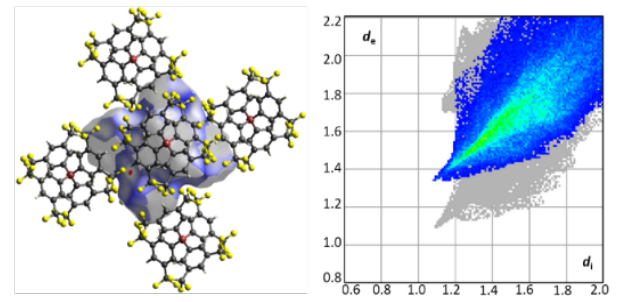

H $\cdots F(62.6 \%)$
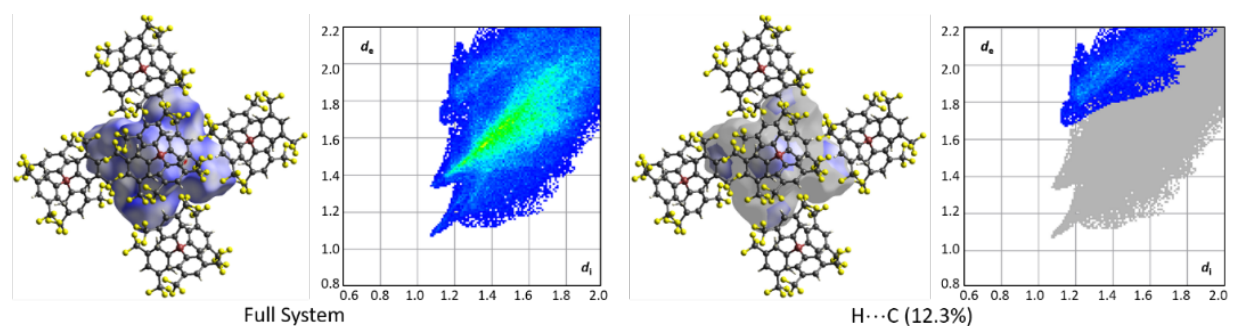

$\mathrm{H} \cdots \mathrm{C}(12.3 \%)$

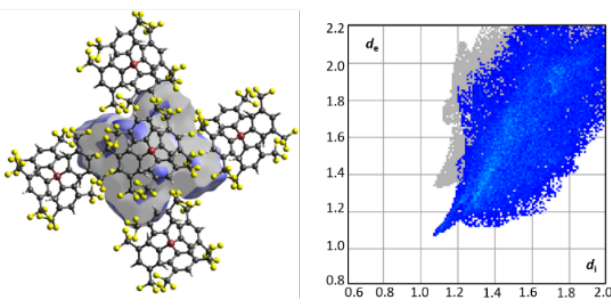

H...H (25.4\%)

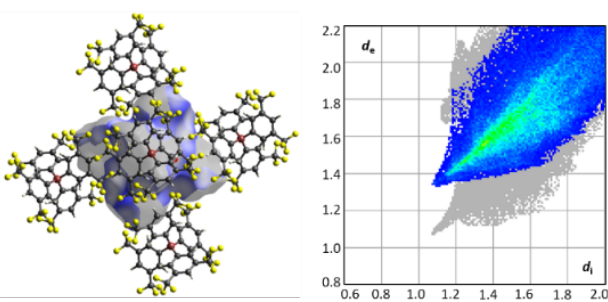

H...F (62.3\%)

Figure S83: Hirshfeld Surfaces of [1-(2-methylbutane)][BAr $\left.{ }_{4}\right]$ (partially Figure S84: Hirshfeld Surfaces of [1-(2-methylbutane)][BAr $\left.{ }_{4}\right]$ (fully optimised) showing normalised distance and associated fingerprint plots. optimised) showing normalised distance and associated fingerprint plots. Percentage of each type of contact is shown in brackets. Percentage of each type of contact is shown in brackets. 


\section{S.5.4. $\left[1-\right.$ hexane $^{2}\left[\mathrm{BAr}_{4}{ }_{4}\right]$}

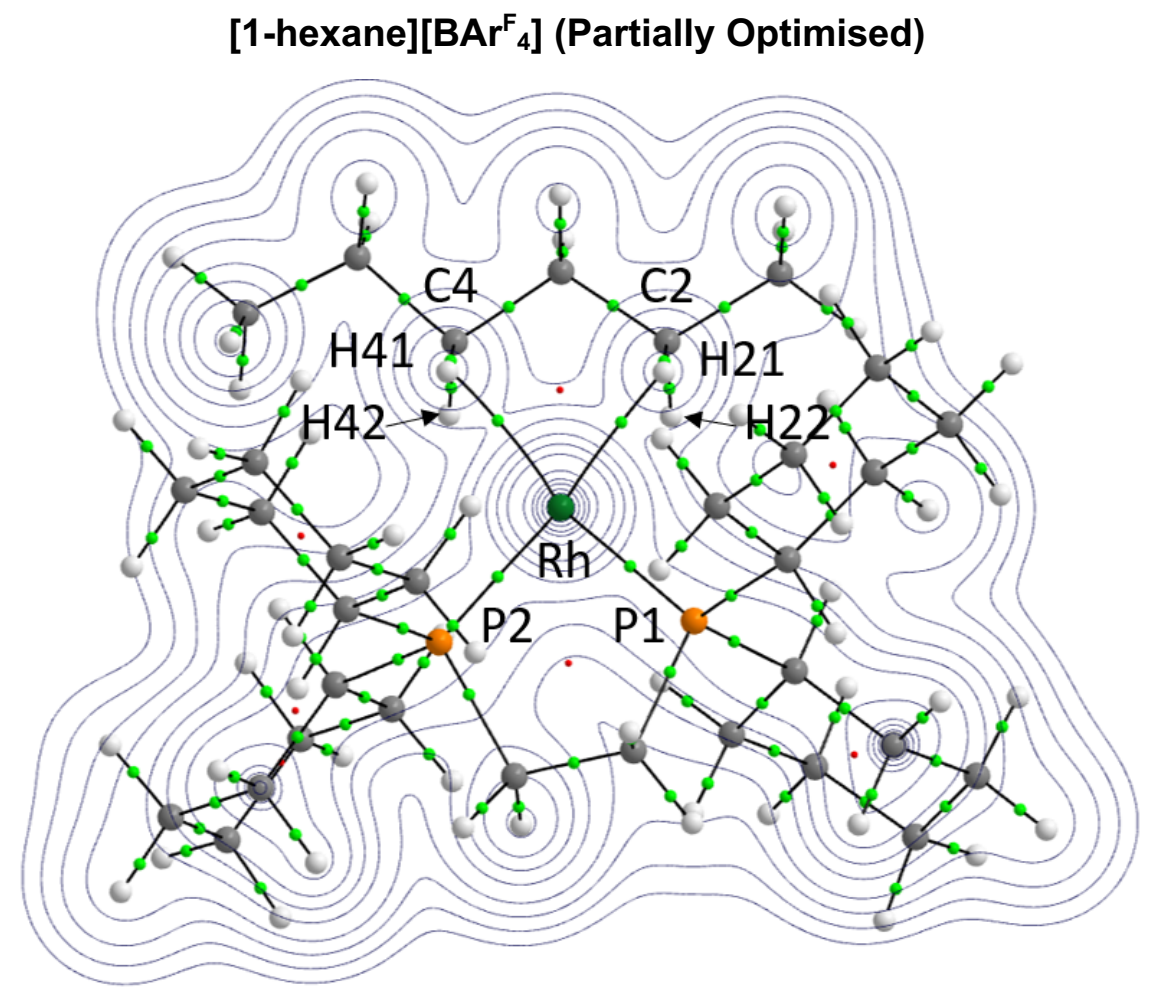

Table S13: QTAIM BCP data and bond lengths for key bonds.

\begin{tabular}{|c|c|c|c|c|}
\hline \multirow[b]{2}{*}{$\begin{array}{l}\text { Bond } \\
\text { Path }\end{array}$} & \multicolumn{4}{|c|}{ Interatomic } \\
\hline & $\begin{array}{c}\text { Distance } \\
\text { (A) }\end{array}$ & $\begin{array}{c}\rho \\
\left(\mathrm{e} b o h r^{-3}\right)\end{array}$ & $\begin{array}{c}\nabla^{2} \rho \\
\left(\text { e bohr } r^{-5}\right)\end{array}$ & $H(r)$ \\
\hline $\mathrm{C} 1-\mathrm{H} 1$ & 1.131 & 0.2489 & -0.7298 & -0.2304 \\
\hline H1-Rh & 2.065 & 0.0418 & 0.1590 & -0.0031 \\
\hline C1-Rh & 2.550 & & No BCP & \\
\hline Rh-P1 & 2.200 & 0.1204 & 0.0722 & -0.0605 \\
\hline $\mathrm{C} 2-\mathrm{H} 2$ & 1.142 & 0.2421 & -0.6781 & -0.2185 \\
\hline H2-Rh & 1.981 & 0.0488 & 0.1794 & -0.0060 \\
\hline C2-Rh & 2.527 & & No BCP & \\
\hline Rh-P2 & 2.191 & 0.1225 & 0.0617 & -0.0631 \\
\hline
\end{tabular}

Figure S85: QTAIM molecular graph of [1-hexane][BAr ${ }_{4}$ ] (partially optimised) with electron density contours in the H41-Rh-H21 plane. 


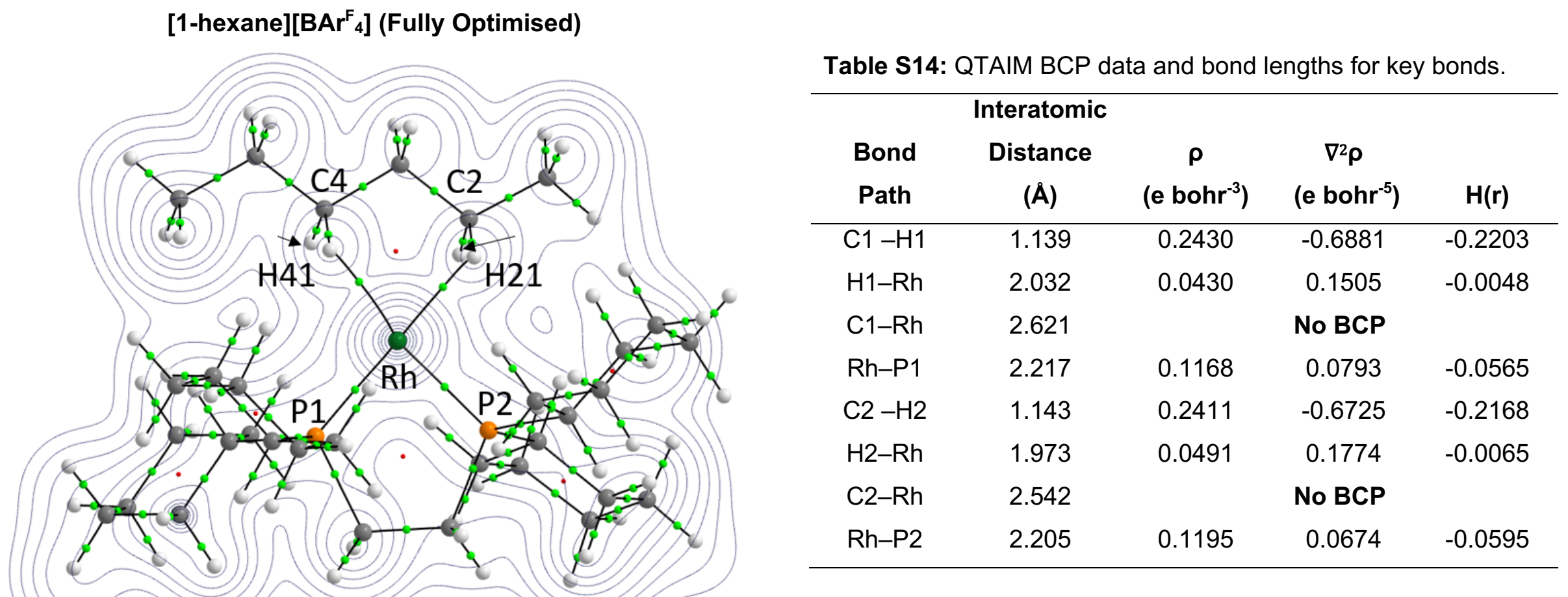

Figure S86: QTAIM molecular graph of [1-hexane][BAr ${ }_{4}$ ] (fully optimised) with electron density contours in the H41-Rh-H21 plane. 

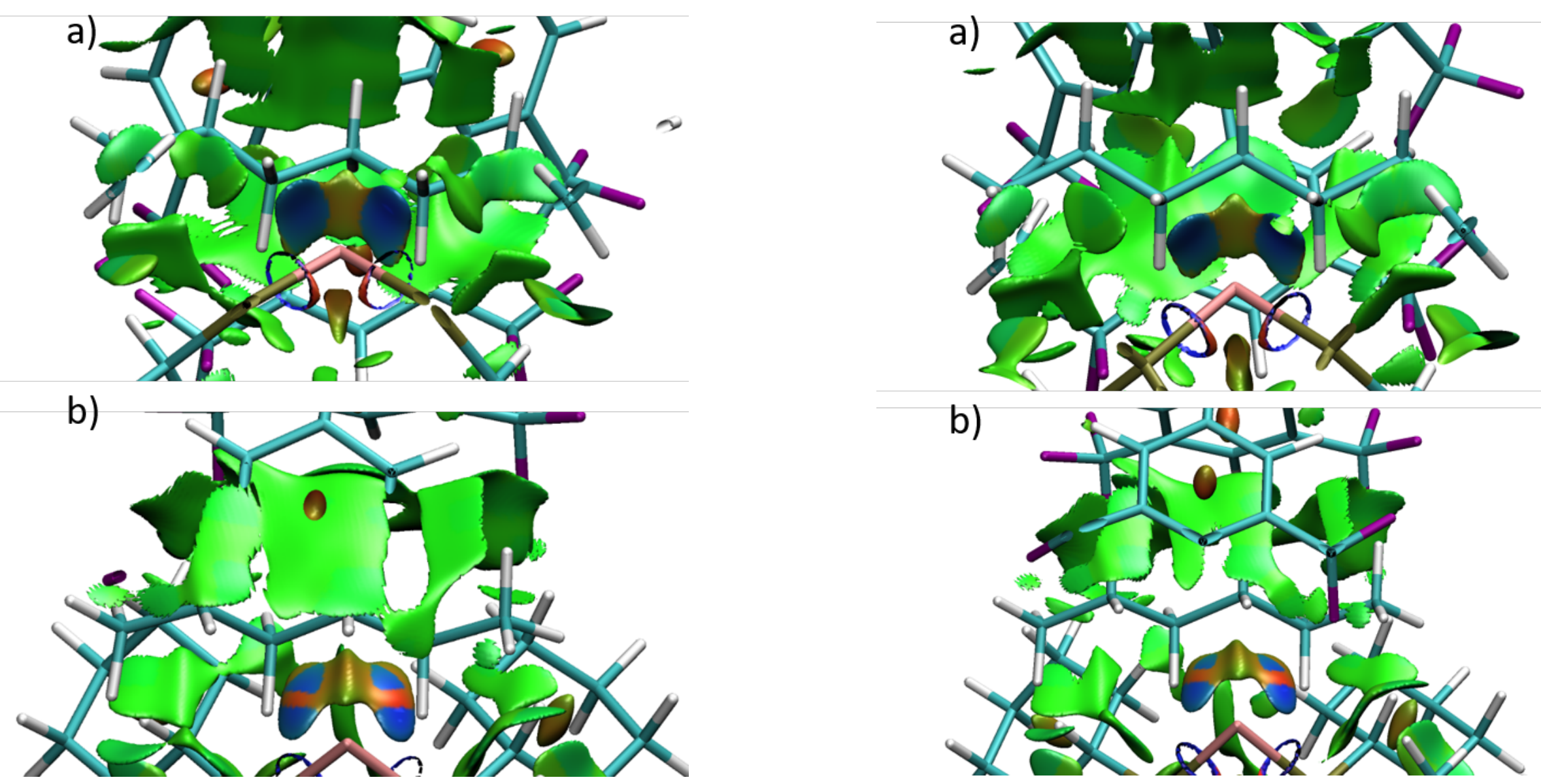

Figure S87: a) $\mathrm{NCl}$ Plot of [1-hexane][BArF ${ }_{4}$ ] (partially optimised) with Figure S88: a) $\mathrm{NCl}$ Plot of [1-hexane][BAr ${ }_{4}$ ] (fully optimised) with isosurfaces generated for $s=0.3$ au and $-0.07<\rho<0.07$ au. View from Rh isosurfaces generated for $s=0.3$ au and $-0.07<\rho<0.07$ au. View from Rh looking towards the alkane. b) View from above the alkane looking towards looking towards the alkane. b) View from above the alkane looking towards Rh. Rh. 
Table S15: NBO data from [1-hexane][BAr ${ }_{4}$ ] (partially optimised) showing the donating and accepting orbitals occupation and the $2^{\text {nd }}$ order perturbation theory interaction energy.

\begin{tabular}{|c|c|c|c|c|}
\hline $\begin{array}{c}\text { Donating } \\
\text { Orbital }\end{array}$ & Occupation & $\begin{array}{c}\text { Accepting } \\
\text { Orbital }\end{array}$ & Occupation & $\begin{array}{c}\text { Energy } \\
\text { (kcal/mol) }\end{array}$ \\
\hline \multicolumn{5}{|c|}{$\mathrm{C} 4-\mathrm{H} 41$} \\
\hline$\sigma(\mathrm{C}-\mathrm{H})$ & 1.92 & $\begin{array}{c}\sigma^{*}(\mathrm{Rh}- \\
\left.P_{\text {trans }}\right)\end{array}$ & 0.26 & 10.14 \\
\hline$\sigma(\mathrm{C}-\mathrm{H})$ & 1.92 & $\begin{array}{c}\sigma^{*}(\mathrm{Rh}- \\
\left.\mathrm{P}_{\mathrm{CIS}}\right)\end{array}$ & 0.25 & 0.10 \\
\hline LP (Rh) & 1.96 & $\sigma^{*}(\mathrm{C}-\mathrm{H})$ & 0.04 & 0.79 \\
\hline LP (Rh) & 1.94 & $\sigma^{*}(\mathrm{C}-\mathrm{H})$ & 0.04 & 1.42 \\
\hline LP (Rh) & 1.89 & $\sigma^{*}(\mathrm{C}-\mathrm{H})$ & 0.04 & 2.18 \\
\hline$\sigma\left(\mathrm{Rh}-\mathrm{P}_{\text {cis }}\right)$ & 1.83 & $\sigma^{*}(\mathrm{C}-\mathrm{H})$ & 0.04 & 1.79 \\
\hline $\begin{array}{c}\sigma(\mathrm{Rh}- \\
\left.\mathrm{P}_{\text {trans }}\right)\end{array}$ & 1.83 & $\sigma^{*}(\mathrm{C}-\mathrm{H})$ & 0.04 & 0.93 \\
\hline \multicolumn{5}{|c|}{$\mathrm{C} 4-\mathrm{H} 42$} \\
\hline$\sigma(\mathrm{C}-\mathrm{H})$ & 1.96 & $\begin{array}{c}\sigma^{*}(\mathrm{Rh}- \\
\left.\mathrm{P}_{\text {trans }}\right)\end{array}$ & 0.25 & 2.11 \\
\hline LP (Rh) & 1.96 & $\sigma^{*}(\mathrm{C}-\mathrm{H})$ & 0.02 & 0.30 \\
\hline $\mathrm{LP}(\mathrm{Rh})$ & 1.94 & $\sigma^{*}(\mathrm{C}-\mathrm{H})$ & 0.02 & 0.27 \\
\hline LP (Rh) & 1.90 & $\sigma^{*}(\mathrm{C}-\mathrm{H})$ & 0.02 & 0.14 \\
\hline$\sigma\left(R h-P_{\text {cis }}\right)$ & 1.83 & $\sigma^{*}(C-H)$ & 0.02 & 0.26 \\
\hline$\sigma(\mathrm{Rh}-$ & 1.83 & $\sigma^{*}(\mathrm{C}-\mathrm{H})$ & 0.02 & 0.18 \\
\hline
\end{tabular}

\begin{tabular}{ccccc}
$\left.P_{\text {trans }}\right)$ & \multicolumn{5}{c}{ C2-H21 } \\
\hline$\sigma(\mathrm{C}-\mathrm{H})$ & 1.90 & $\sigma^{*}(\mathrm{Rh}-$ & 0.26 & 13.78 \\
& & $\left.\mathrm{P}_{\text {trans }}\right)$ & \\
LP $(\mathrm{Rh})$ & 1.96 & $\sigma^{*}(\mathrm{C}-\mathrm{H})$ & 0.04 & 0.10 \\
LP $(\mathrm{Rh})$ & 1.94 & $\sigma^{*}(\mathrm{C}-\mathrm{H})$ & 0.04 & 2.34 \\
LP $(\mathrm{Rh})$ & 1.89 & $\sigma^{*}(\mathrm{C}-\mathrm{H})$ & 0.04 & 2.98 \\
$\sigma\left(\mathrm{Rh}-\mathrm{P}_{\text {cis }}\right)$ & 1.83 & $\sigma^{*}(\mathrm{C}-\mathrm{H})$ & 0.04 & 2.43 \\
$\sigma(\mathrm{Rh}-$ & 1.83 & $\sigma^{*}(\mathrm{C}-\mathrm{H})$ & 0.04 & 0.96 \\
$\left.\mathrm{P}_{\text {trans }}\right)$ & & & & \\
\hline & & $\mathrm{C} 2-\mathrm{H} 22$ & & \\
\hline$\sigma(\mathrm{C}-\mathrm{H})$ & 1.96 & $\sigma^{*}(\mathrm{Rh}-$ & 0.26 \\
& & $\left.\mathrm{P}_{\text {trans }}\right)$ & & 2.38 \\
LP $(\mathrm{Rh})$ & 1.96 & $\sigma^{*}(\mathrm{C}-\mathrm{H})$ & 0.02 & 0.83 \\
LP $(\mathrm{Rh})$ & 1.94 & $\sigma^{*}(\mathrm{C}-\mathrm{H})$ & 0.02 & 0.11 \\
LP $(\mathrm{Rh})$ & 1.90 & $\sigma^{*}(\mathrm{C}-\mathrm{H})$ & 0.02 & 0.15 \\
$\sigma\left(\mathrm{Rh}-\mathrm{P}_{\text {cis }}\right)$ & 1.83 & $\sigma^{*}(\mathrm{C}-\mathrm{H})$ & 0.02 & 0.41 \\
$\sigma\left(\mathrm{Rh}-\mathrm{P}_{\text {trans }}\right)$ & 1.83 & $\sigma^{*}(\mathrm{C}-\mathrm{H})$ & 0.02 & 0.28 \\
\hline
\end{tabular}


Table S16: NBO data from [1-hexane][BAr ${ }_{4}$ ] (fully optimised) showing the donating and accepting orbitals occupation and the $2^{\text {nd }}$ order perturbation theory interaction energy.

\begin{tabular}{|c|c|c|c|c|}
\hline $\begin{array}{c}\text { Donating } \\
\text { Orbital }\end{array}$ & Occupation & $\begin{array}{c}\text { Accepting } \\
\text { Orbital }\end{array}$ & Occupation & $\begin{array}{c}\text { Energy } \\
\text { (kcal/mol) }\end{array}$ \\
\hline \multicolumn{5}{|c|}{$\mathrm{C} 4-\mathrm{H} 41$} \\
\hline$\sigma(\mathrm{C}-\mathrm{H})$ & 1.91 & $\begin{array}{c}\sigma^{*}(\mathrm{Rh}- \\
\left.\mathrm{P}_{\text {trans }}\right)\end{array}$ & 0.25 & 11.45 \\
\hline$\sigma(\mathrm{C}-\mathrm{H})$ & 1.91 & $\begin{array}{c}\sigma^{*}(\mathrm{Rh}- \\
\left.\mathrm{P}_{\mathrm{CIS}}\right)\end{array}$ & 0.26 & 0.17 \\
\hline LP (Rh) & 1.96 & $\sigma^{*}(\mathrm{C}-\mathrm{H})$ & 0.04 & 0.49 \\
\hline LP (Rh) & 1.94 & $\sigma^{*}(\mathrm{C}-\mathrm{H})$ & 0.04 & 1.55 \\
\hline LP (Rh) & 1.90 & $\sigma^{*}(\mathrm{C}-\mathrm{H})$ & 0.04 & 0.23 \\
\hline $\mathrm{LP}(\mathrm{Rh})$ & 1.89 & $\sigma^{*}(\mathrm{C}-\mathrm{H})$ & 0.04 & 2.26 \\
\hline$\sigma\left(\mathrm{Rh}-\mathrm{P}_{\text {cis }}\right)$ & 1.91 & $\sigma^{*}(\mathrm{C}-\mathrm{H})$ & 0.04 & 2.44 \\
\hline \multicolumn{5}{|l|}{$\left.\mathrm{P}_{\text {trans }}\right)$} \\
\hline \multicolumn{5}{|c|}{$\mathrm{C} 4-\mathrm{H} 42$} \\
\hline$\sigma(\mathrm{C}-\mathrm{H})$ & 1.97 & $\begin{array}{c}\sigma^{*}(\mathrm{Rh}- \\
\left.\mathrm{P}_{\text {trans }}\right)\end{array}$ & 0.25 & 1.35 \\
\hline LP (Rh) & 1.97 & $\sigma^{*}(\mathrm{C}-\mathrm{H})$ & 0.02 & 0.32 \\
\hline LP (Rh) & 1.94 & $\sigma^{*}(\mathrm{C}-\mathrm{H})$ & 0.02 & 0.18 \\
\hline LP (Rh) & 1.90 & $\sigma^{*}(\mathrm{C}-\mathrm{H})$ & 0.02 & 0.08 \\
\hline$\sigma\left(R h-P_{\text {cis }}\right)$ & 1.83 & $\sigma^{*}(\mathrm{C}-\mathrm{H})$ & 0.02 & 0.27 \\
\hline
\end{tabular}

\begin{tabular}{ccccc}
$\sigma\left(\mathrm{Rh}-\mathrm{P}_{\text {trans }}\right)$ & 1.83 & $\sigma^{*}(\mathrm{C}-\mathrm{H})$ & 0.02 & 0.22 \\
\hline$\sigma(\mathrm{C}-\mathrm{H})$ & 1.90 & $\sigma^{*}(\mathrm{Rh}-\mathrm{H} 21$ & 0.26 & 14.12 \\
& & $\left.\mathrm{P}_{\text {trans }}\right)$ & & \\
LP $(\mathrm{Rh})$ & 1.97 & $\sigma^{*}(\mathrm{C}-\mathrm{H})$ & 0.04 & 0.08 \\
LP $(\mathrm{Rh})$ & 1.94 & $\sigma^{*}(\mathrm{C}-\mathrm{H})$ & 0.04 & 2.44 \\
LP $(\mathrm{Rh})$ & 1.90 & $\sigma^{*}(\mathrm{C}-\mathrm{H})$ & 0.04 & 0.20 \\
LP $(\mathrm{Rh})$ & 1.89 & $\sigma^{*}(\mathrm{C}-\mathrm{H})$ & 0.04 & 2.91 \\
$\sigma\left(\mathrm{Rh}-\mathrm{P}_{\text {cis }}\right)$ & 1.83 & $\sigma^{*}(\mathrm{C}-\mathrm{H})$ & 0.04 & 2.44 \\
$\sigma(\mathrm{Rh}-$ & 1.83 & $\sigma^{*}(\mathrm{C}-\mathrm{H})$ & 0.04 & 0.95 \\
$\left.\mathrm{P}_{\text {trans }}\right)$ & & & & \\
\hline & & $\mathrm{C} 2-\mathrm{H} 22$ & & \\
\hline$\sigma(\mathrm{C}-\mathrm{H})$ & 1.97 & $\sigma^{*}(\mathrm{Rh}-$ & 0.26 & 2.15 \\
& & $\left.\mathrm{P}_{\text {trans }}\right)$ & & \\
LP (Rh) & 1.97 & $\sigma^{*}(\mathrm{C}-\mathrm{H})$ & 0.02 & 0.81 \\
LP (Rh) & 1.94 & $\sigma^{*}(\mathrm{C}-\mathrm{H})$ & 0.02 & 0.11 \\
LP (Rh) & 1.90 & $\sigma^{*}(\mathrm{C}-\mathrm{H})$ & 0.02 & 0.14 \\
$\sigma\left(\mathrm{Rh}-\mathrm{P}_{\text {cis }}\right)$ & 1.83 & $\sigma^{*}(\mathrm{C}-\mathrm{H})$ & 0.02 & 0.39 \\
$\sigma(\mathrm{Rh}-$ & 1.83 & $\sigma^{*}(\mathrm{C}-\mathrm{H})$ & 0.02 & 0.25 \\
$\left.P_{\text {trans }}\right)$ & & & & \\
\hline
\end{tabular}




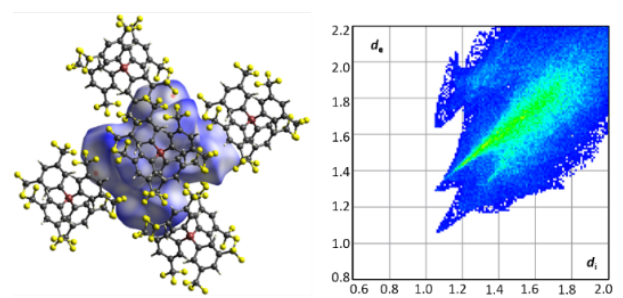

Full System

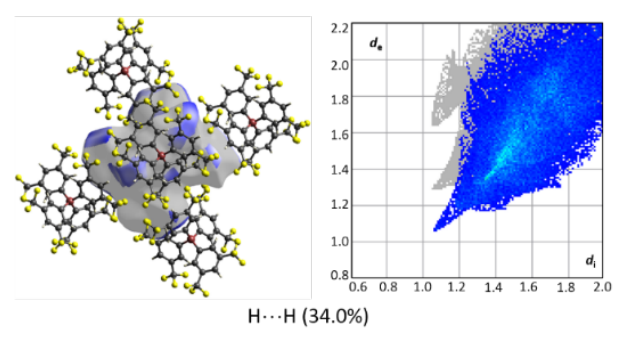

Figure S89: Hirshfeld Surfaces of [1-hexane][BAr ${ }_{4}$ ] (partially optimised) showing normalised distance and associated fingerprint plots. Percentage of showing each type of
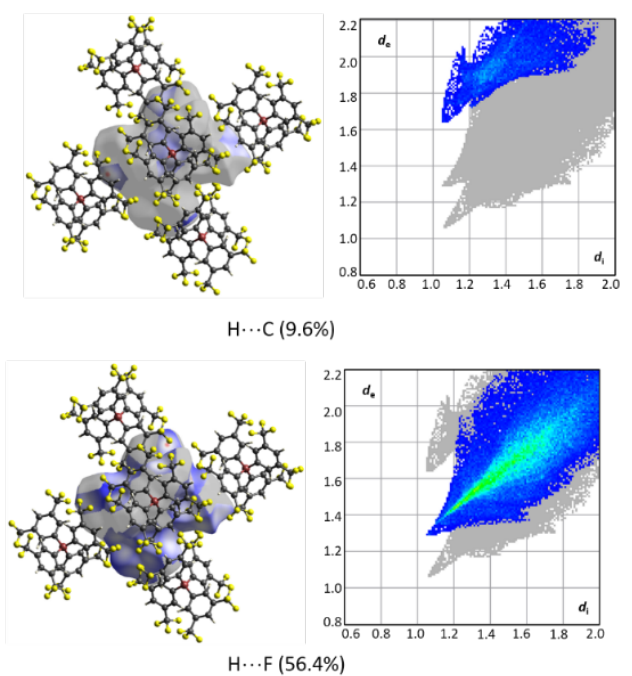

$H \cdots C(9.6 \%)$

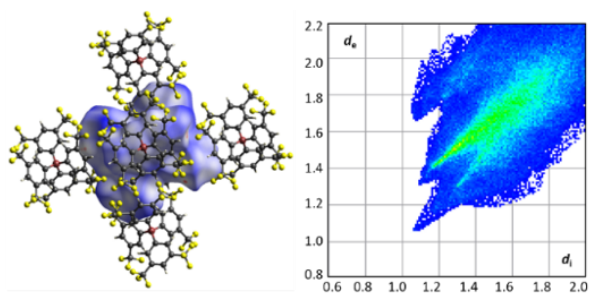

Full System

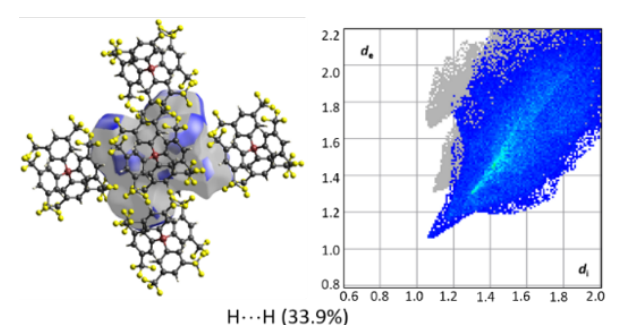

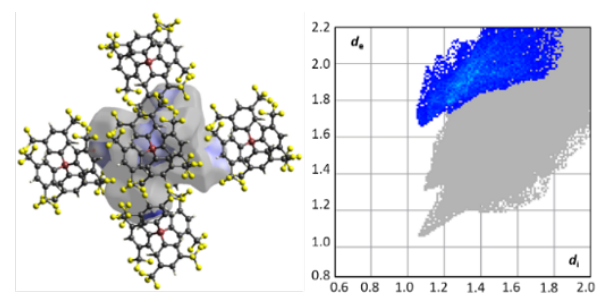

$\mathrm{H} \cdots \mathrm{C}(9.6 \%)$

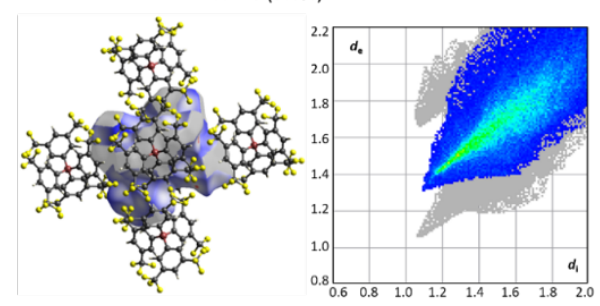

$H \cdots F(56.4 \%)$

Figure S90: Hirshfeld Surfaces of [1-hexane][BArF ${ }_{4}$ ] (fully optimised) 


\section{[1-(3-methylpentane)][BArF $\left.{ }_{4}\right]$ (Partially Optimised)}

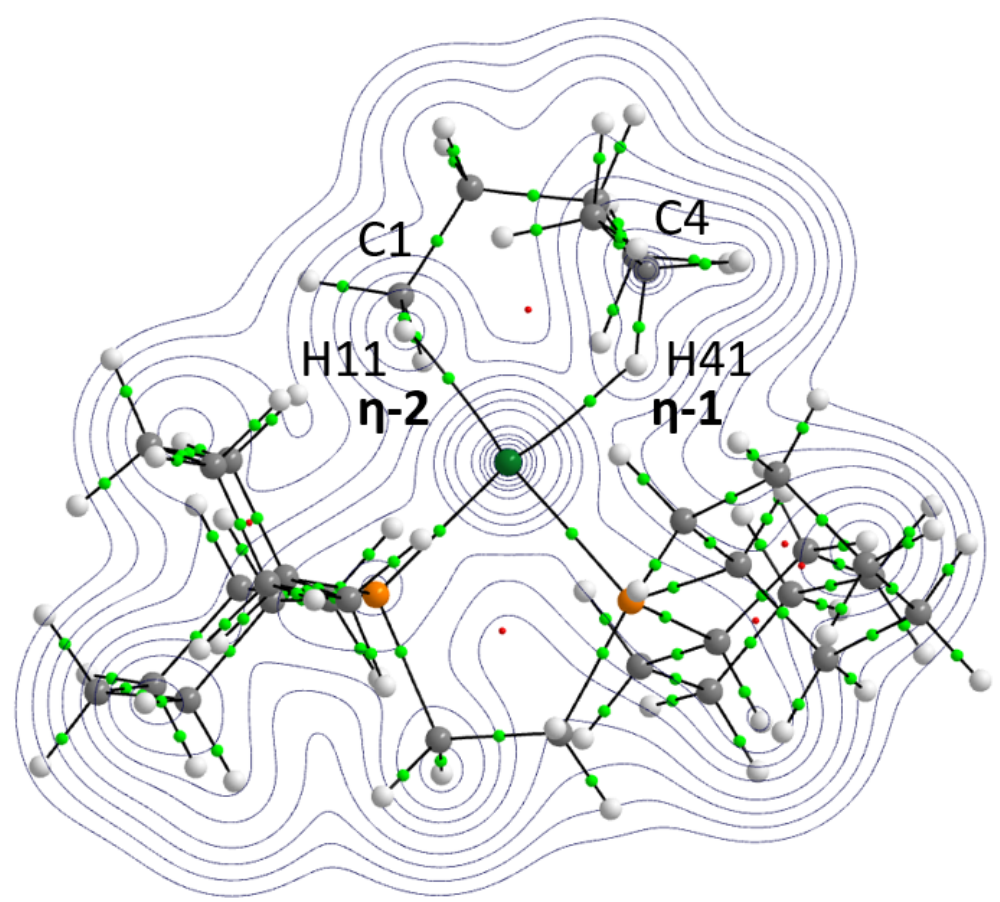

Figure S91: QTAIM molecular graph of [1-(3-methylpentane)][BArF ${ }_{4}$ ] (partially optimised) with electron density contours in the $\mathrm{H} 11-\mathrm{Rh}-\mathrm{H} 41$ plane.
Table S17: QTAIM BCP data and bond lengths for key bonds.

\begin{tabular}{|c|c|c|c|c|}
\hline & Interatomic & & & \\
\hline $\begin{array}{l}\text { Bond } \\
\text { Path }\end{array}$ & $\begin{array}{c}\text { Distance } \\
\text { (A) }\end{array}$ & $\begin{array}{c}\rho \\
\left(\mathrm{e} b o h r^{-3}\right)\end{array}$ & $\begin{array}{c}\nabla^{2} \rho \\
\left(\text { e bohr } r^{-5}\right)\end{array}$ & $H(r)$ \\
\hline $\mathrm{C} 1-\mathrm{H} 11$ & 1.132 & 0.2472 & -0.7132 & -0.2282 \\
\hline $\mathrm{H} 11-\mathrm{Rh}$ & 1.978 & 0.0520 & 0.2059 & -0.0051 \\
\hline C1-Rh & 2.429 & & No $B C P$ & \\
\hline Rh-P1 & 2.196 & 0.1210 & 0.0673 & 0.0615 \\
\hline $\mathrm{C} 4-\mathrm{H} 41$ & 1.136 & 0.2887 & -0.6525 & -0.2045 \\
\hline $\mathrm{H} 41-\mathrm{Rh}$ & 1.885 & 0.0537 & 0.1782 & -0.0104 \\
\hline C4-Rh & 2.788 & & No BCP & \\
\hline Rh-P2 & 2.196 & 0.1217 & 0.0728 & 0.0619 \\
\hline
\end{tabular}




\section{[1-(3-methylpentane)][BAr ${ }_{4}{ }_{4}$ (fully Optimised)}

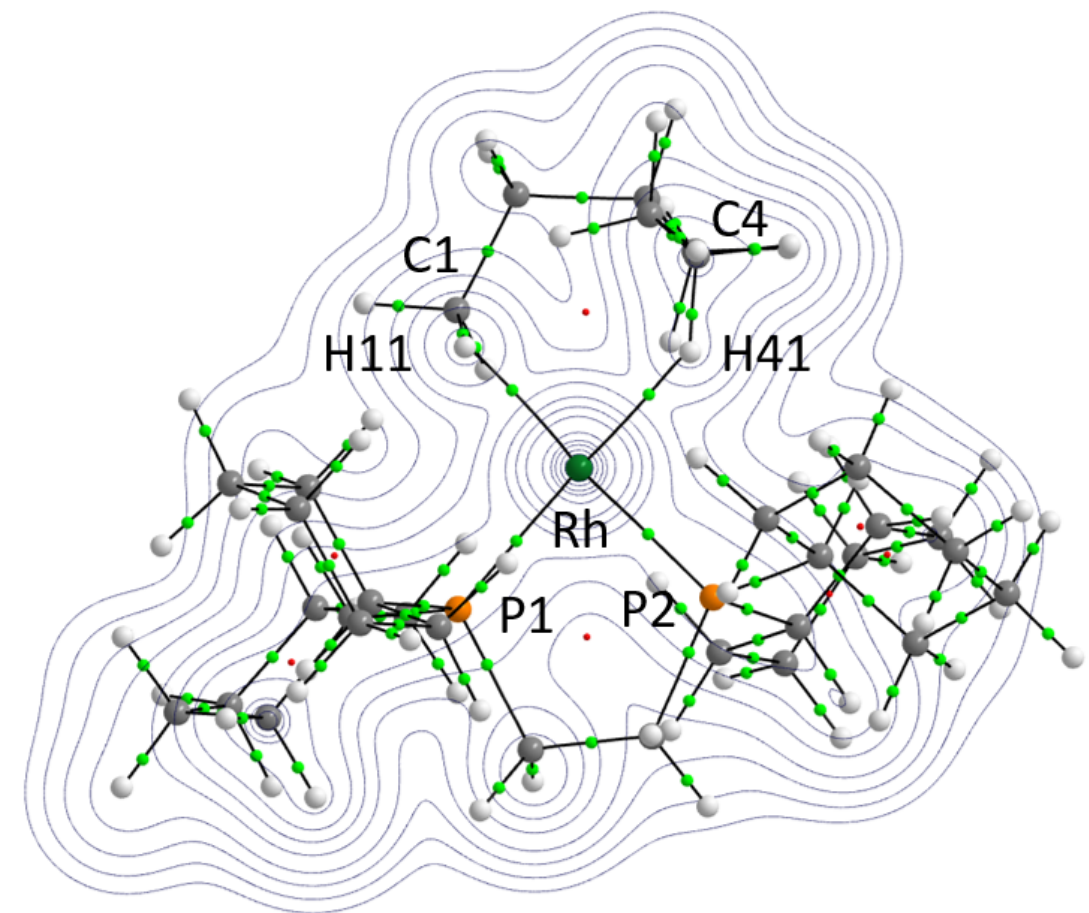

Table S18: QTAIM BCP data and bond lengths for key bonds.

\begin{tabular}{ccccc}
\hline $\begin{array}{c}\text { Bond } \\
\text { Path }\end{array}$ & $\begin{array}{c}\text { Interatomic } \\
\text { Distance } \\
(\mathbf{A})\end{array}$ & $\begin{array}{c}\boldsymbol{\rho} \\
\left(\mathbf{e} \mathbf{b o h h}^{-3}\right)\end{array}$ & $\begin{array}{c}\boldsymbol{\nabla}^{2} \boldsymbol{\rho} \\
(\mathbf{e} \text { bohr }\end{array}$ & $\mathbf{H})$ \\
\hline C1 -H11 & 1.135 & 0.2450 & -0.7003 & -0.2244 \\
H11-Rh & 1.979 & 0.0504 & 0.1963 & -0.0054 \\
C1-Rh & 2.463 & & No BCP & \\
Rh-P1 & 2.200 & 0.1201 & 0.0667 & -0.0607 \\
C4-H41 & 1.141 & 0.2360 & -0.6339 & -0.2094 \\
H41-Rh & 1.928 & 0.0492 & 0.1517 & -0.0074 \\
C4-Rh & 2.891 & & No BCP & \\
Rh-P2 & 2.211 & 0.1185 & 0.0779 & -0.0582 \\
\hline
\end{tabular}

Figure S92: QTAIM molecular graph of [1-(3-methylpentane)][BAr $\left.{ }_{4}\right]$ (fully optimised) with electron density contours in the H11-Rh-H41 plane. 

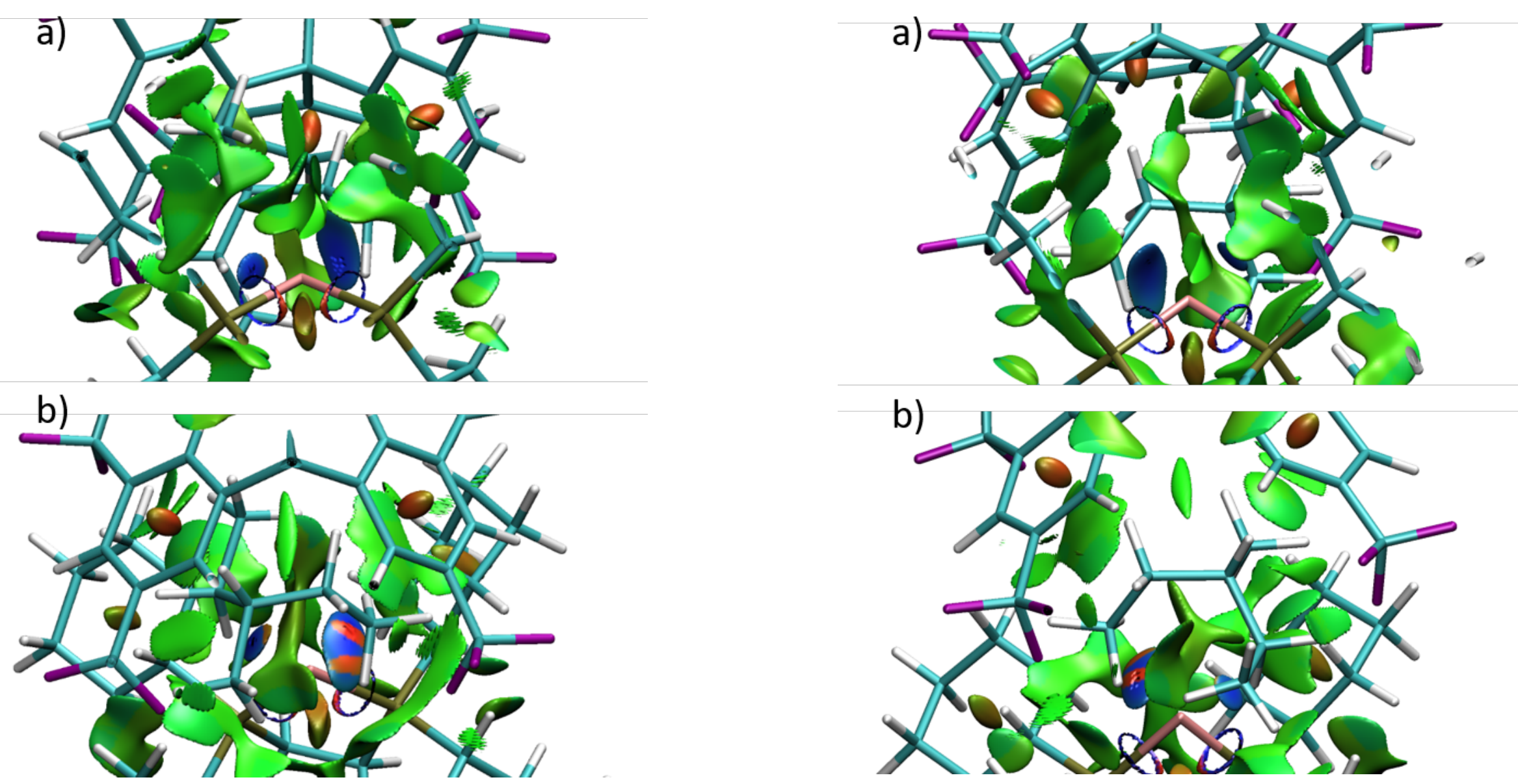

Figure S93: a) NCl Plot of [1-(3-methylpentane)][BAr $\left.{ }_{4}\right]$ (partially optimised) Figure S94: a) NCl Plot of [1-(3-methylpentane)][BAr ${ }_{4}{ }_{4}$ (fully optimised) with isosurfaces generated for $s=0.3$ au and $-0.07<\rho<0.07$ au. View from with isosurfaces generated for $s=0.3$ au and $-0.07<\rho<0.07$ au. View from Rh looking towards the alkane. b) View from above the alkane looking Rh looking towards the alkane. b) View from above the alkane looking towards Rh. towards Rh. 
Table S19: NBO data from [1-(3-methylpentane)][BAr ${ }_{4}$ ] (partially optimised) showing the donating and accepting orbitals occupation and the $2^{\text {nd }}$ order perturbation theory interaction energy.

\begin{tabular}{|c|c|c|c|c|}
\hline $\begin{array}{c}\text { Donating } \\
\text { Orbital }\end{array}$ & Occupation & $\begin{array}{c}\text { Accepting } \\
\text { Orbital }\end{array}$ & Occupation & $\begin{array}{c}\text { Energy } \\
\text { (kcal/mol) }\end{array}$ \\
\hline \multicolumn{5}{|c|}{$\mathrm{C} 1-\mathrm{H} 11$} \\
\hline$\sigma(\mathrm{C}-\mathrm{H})$ & 1.92 & $\begin{array}{c}\sigma^{*}(\mathrm{Rh}- \\
\left.\mathrm{P}_{\text {trans }}\right)\end{array}$ & 0.28 & 13.44 \\
\hline LP (Rh) & 1.96 & $\sigma^{*}(\mathrm{C}-\mathrm{H})$ & 0.04 & 0.24 \\
\hline LP (Rh) & 1.95 & $\sigma^{*}(\mathrm{C}-\mathrm{H})$ & 0.04 & 1.98 \\
\hline LP (Rh) & 1.90 & $\sigma^{*}(\mathrm{C}-\mathrm{H})$ & 0.04 & 0.91 \\
\hline LP (Rh) & 1.89 & $\sigma^{*}(\mathrm{C}-\mathrm{H})$ & 0.04 & 3.05 \\
\hline$\sigma\left(\mathrm{Rh}-\mathrm{P}_{\text {cis }}\right)$ & 1.81 & $\sigma^{*}(\mathrm{C}-\mathrm{H})$ & 0.04 & 2.10 \\
\hline $\begin{array}{c}\sigma(\mathrm{Rh}- \\
\left.\mathrm{P}_{\text {trans }}\right)\end{array}$ & 1.82 & $\sigma^{*}(\mathrm{C}-\mathrm{H})$ & 0.04 & 0.84 \\
\hline \multicolumn{5}{|c|}{$\mathrm{C} 1-\mathrm{H} 12$} \\
\hline$\sigma(\mathrm{C}-\mathrm{H})$ & 1.95 & $\begin{array}{c}\sigma^{*}(\mathrm{Rh}- \\
\left.\mathrm{P}_{\text {trans }}\right)\end{array}$ & 0.28 & 5.90 \\
\hline $\mathrm{LP}(\mathrm{Rh})$ & 1.96 & $\sigma^{*}(\mathrm{C}-\mathrm{H})$ & 0.03 & 2.03 \\
\hline LP (Rh) & 1.95 & $\sigma^{*}(\mathrm{C}-\mathrm{H})$ & 0.03 & 0.12 \\
\hline LP (Rh) & 1.90 & $\sigma^{*}(\mathrm{C}-\mathrm{H})$ & 0.03 & 0.69 \\
\hline LP (Rh) & 1.89 & $\sigma^{*}(\mathrm{C}-\mathrm{H})$ & 0.03 & 0.34 \\
\hline$\sigma\left(\mathrm{Rh}-\mathrm{P}_{\text {cis }}\right)$ & 0.82 & $\sigma^{*}(\mathrm{C}-\mathrm{H})$ & 0.03 & 0.96 \\
\hline
\end{tabular}

\begin{tabular}{ccccc}
$\begin{array}{c}\sigma(\mathrm{Rh}- \\
\left.\mathrm{P}_{\text {trans }}\right)\end{array}$ & 0.81 & $\sigma^{*}(\mathrm{C}-\mathrm{H})$ & 0.03 & 0.55 \\
\hline$\sigma(\mathrm{C}-\mathrm{H})$ & 1.89 & $\sigma^{*}(\mathrm{Rh}-$ & 0.28 & 14.23 \\
& & $\left.\mathrm{P}_{\text {trans }}\right)$ & & \\
\hline LP (Rh) & 1.96 & $\sigma^{*}(\mathrm{C}-\mathrm{H})$ & 0.04 & 0.11 \\
LP (Rh) & 1.95 & $\sigma^{*}(\mathrm{C}-\mathrm{H})$ & 0.04 & 0.38 \\
LP (Rh) & 1.90 & $\sigma^{*}(\mathrm{C}-\mathrm{H})$ & 0.04 & 0.96 \\
LP (Rh) & 1.89 & $\sigma^{*}(\mathrm{C}-\mathrm{H})$ & 0.04 & 1.19 \\
$\sigma\left(\mathrm{Rh}-\mathrm{P}_{\text {cis }}\right)$ & 1.82 & $\sigma^{*}(\mathrm{C}-\mathrm{H})$ & 0.04 & 7.01 \\
$\sigma(\mathrm{Rh}-$ & 1.81 & $\sigma^{*}(\mathrm{C}-\mathrm{H})$ & 0.04 & 2.89 \\
$\left.\mathrm{P}_{\text {trans }}\right)$ & & & & \\
\hline
\end{tabular}


Table S20: NBO data from [1-(3-methylpentane)][BAr ${ }_{4}$ ] (fully optimised) showing the donating and accepting orbitals occupation and the $2^{\text {nd }}$ order perturbation theory interaction energy. Selected donor-acceptor interactions are shown below.

\begin{tabular}{|c|c|c|c|c|}
\hline $\begin{array}{c}\text { Donating } \\
\text { Orbital }\end{array}$ & Occupation & $\begin{array}{c}\text { Accepting } \\
\text { Orbital }\end{array}$ & Occupation & $\begin{array}{c}\text { Energy } \\
\text { (kcal/mol) }\end{array}$ \\
\hline \multicolumn{5}{|c|}{$\mathrm{C} 1-\mathrm{H} 11$} \\
\hline$\sigma(\mathrm{C}-\mathrm{H})$ & 1.91 & $\begin{array}{c}\sigma^{*}(\mathrm{Rh}- \\
\left.\mathrm{P}_{\text {trans }}\right)\end{array}$ & 0.28 & 13.75 \\
\hline LP (Rh) & 1.96 & $\sigma^{*}(\mathrm{C}-\mathrm{H})$ & 0.03 & 0.14 \\
\hline $\mathrm{LP}(\mathrm{Rh})$ & 1.94 & $\sigma^{*}(\mathrm{C}-\mathrm{H})$ & 0.03 & 2.07 \\
\hline LP (Rh) & 1.90 & $\sigma^{*}(\mathrm{C}-\mathrm{H})$ & 0.03 & 0.39 \\
\hline LP (Rh) & 1.89 & $\sigma^{*}(\mathrm{C}-\mathrm{H})$ & 0.03 & 3.54 \\
\hline$\sigma\left(\mathrm{Rh}-\mathrm{P}_{\text {cis }}\right)$ & 0.82 & $\sigma^{*}(\mathrm{C}-\mathrm{H})$ & 0.03 & 2.16 \\
\hline $\begin{array}{c}\sigma(\mathrm{Rh}- \\
\left.\mathrm{P}_{\text {trans }}\right)\end{array}$ & 0.82 & $\sigma^{*}(\mathrm{C}-\mathrm{H})$ & 0.03 & 0.85 \\
\hline \multicolumn{5}{|c|}{$\mathrm{C} 1-\mathrm{H} 12$} \\
\hline$\sigma(\mathrm{C}-\mathrm{H})$ & 1.96 & $\begin{array}{c}\sigma^{*}(\mathrm{Rh}- \\
\left.\mathrm{P}_{\text {trans }}\right)\end{array}$ & 0.28 & 4.83 \\
\hline LP (Rh) & 1.96 & $\sigma^{*}(\mathrm{C}-\mathrm{H})$ & 0.02 & 1.85 \\
\hline $\mathrm{LP}(\mathrm{Rh})$ & 1.94 & $\sigma^{*}(\mathrm{C}-\mathrm{H})$ & 0.02 & 0.14 \\
\hline LP (Rh) & 1.90 & $\sigma^{*}(\mathrm{C}-\mathrm{H})$ & 0.02 & 0.32 \\
\hline $\mathrm{LP}(\mathrm{Rh})$ & 1.89 & $\sigma^{*}(\mathrm{C}-\mathrm{H})$ & 0.02 & 0.41 \\
\hline
\end{tabular}

\begin{tabular}{ccccc}
$\sigma\left(\mathrm{Rh}-\mathrm{P}_{\text {cis }}\right)$ & 0.82 & $\sigma^{*}(\mathrm{C}-\mathrm{H})$ & 0.02 & 0.82 \\
$\sigma(\mathrm{Rh}-$ & 0.82 & $\sigma^{*}(\mathrm{C}-\mathrm{H})$ & 0.02 & 0.51 \\
$\left.\mathrm{P}_{\text {trans }}\right)$ & & & \\
\hline & & $\mathrm{C} 4-\mathrm{H} 41$ & \\
\hline$\sigma(\mathrm{C}-\mathrm{H})$ & 1.89 & $\sigma^{*}(\mathrm{Rh}-$ & 0.28 & 12.61 \\
& & $\left.\mathrm{P}_{\text {trans }}\right)$ & \\
$\sigma(\mathrm{C}-\mathrm{H})$ & 1.89 & $\sigma^{*}\left(\mathrm{Rh}-\mathrm{P}_{\text {cis }}\right)$ & 0.28 & 0.07 \\
$\mathrm{LP}(\mathrm{Rh})$ & 1.94 & $\sigma^{*}(\mathrm{C}-\mathrm{H})$ & 0.04 & 1.22 \\
$\mathrm{LP}(\mathrm{Rh})$ & 1.90 & $\sigma^{*}(\mathrm{C}-\mathrm{H})$ & 0.04 & 0.16 \\
$\mathrm{LP}(\mathrm{Rh})$ & 1.89 & $\sigma^{*}(\mathrm{C}-\mathrm{H})$ & 0.04 & 0.98 \\
$\sigma\left(\mathrm{Rh}-\mathrm{P}_{\text {cis }}\right)$ & 1.82 & $\sigma^{*}(\mathrm{C}-\mathrm{H})$ & 0.04 & $6.25^{\mathrm{a}}$ \\
$\sigma(\mathrm{Rh}-$ & 1.82 & $\sigma^{*}(\mathrm{C}-\mathrm{H})$ & 0.04 & $3.31^{\mathrm{b}}$ \\
$\left.\mathrm{P}_{\text {trans }}\right)$ & & & \\
\hline
\end{tabular}

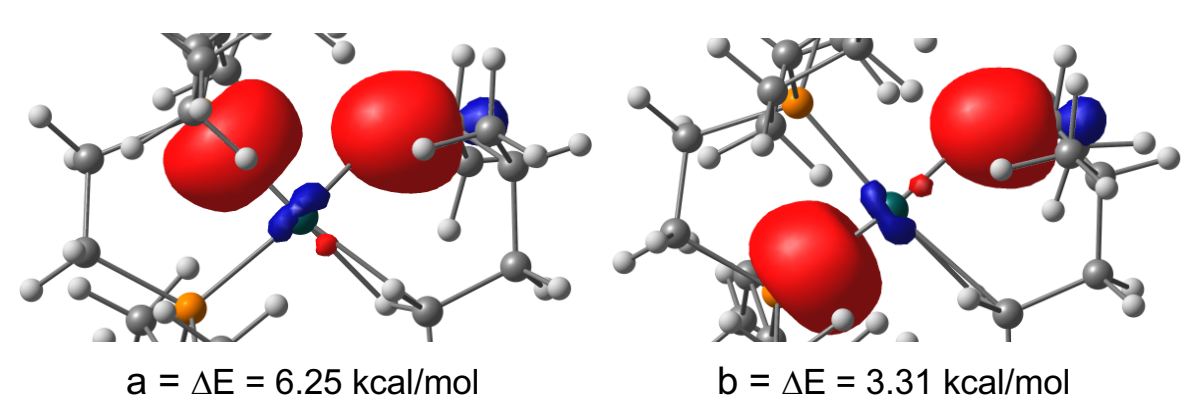




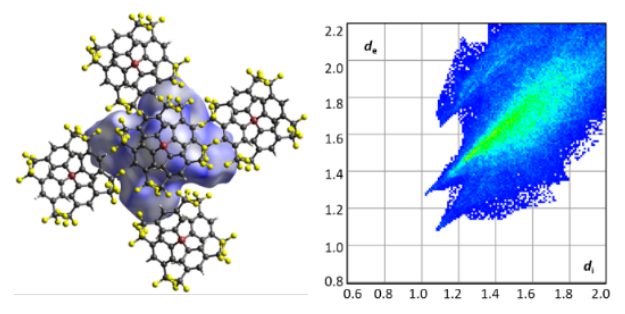

Full System

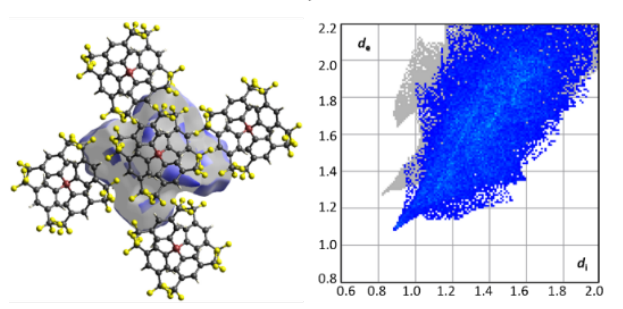

$\mathrm{H} \cdots \mathrm{H}(26.8 \%)$

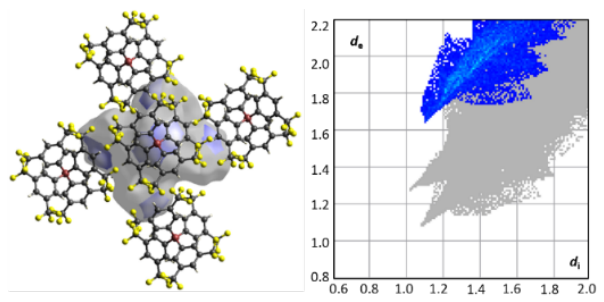

$H \cdots C(12.1 \%)$

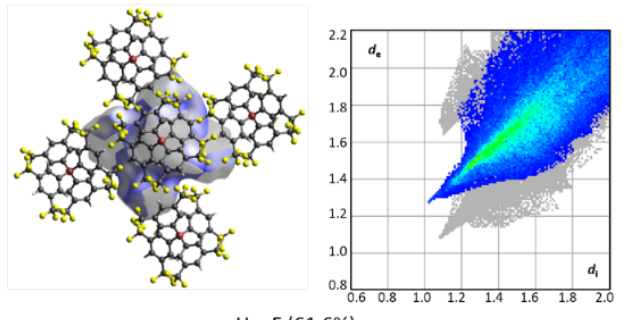

H...F $(61.6 \%)$
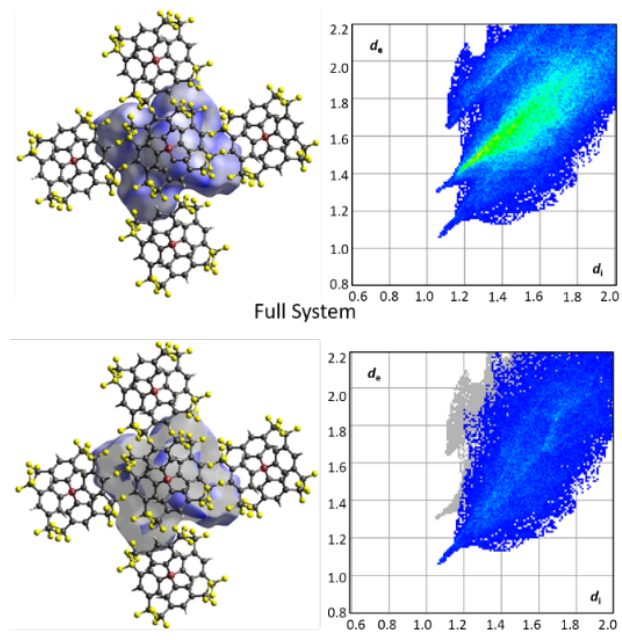

H...H (26.9\%)
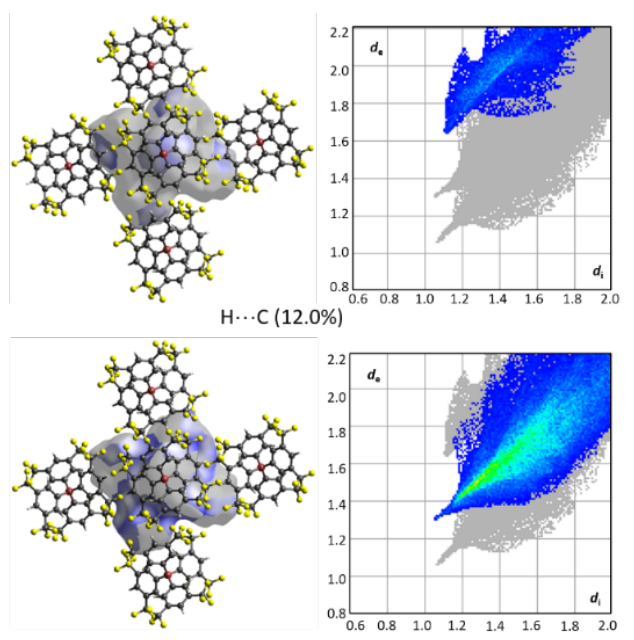

H...F $(61.1 \%)$

Figure S95: Hirshfeld Surfaces of [1-(3-methylpentane)][BAr ${ }_{4}$ ] (partially Figure S96: Hirshfeld Surfaces of [1-(3-methylpentane)][BAr ${ }_{4}$ ] (fully optimised) showing normalised distance and associated fingerprint plots. Percentage of each type of contact is shown in brackets.

optimised) showing normalised distance and associated fingerprint plots. Percentage of each type of contact is shown in brackets. 


\section{S.5.6. [1-cyclohexane][BAr ${ }_{4}$ ]}

Note: The structure of $\left[\mathrm{Rh}\left(\mathrm{Cy}_{2} \mathrm{PCH}_{2} \mathrm{CH}_{2} \mathrm{PCy}\right)\left(\mathrm{C}_{6} \mathrm{H}_{12}\right)\right]\left[\mathrm{BAr}_{4}{ }_{4}\right]$, [1-cylohexane][BAr$\left.{ }_{4}\right]$ has been previous reported. ${ }^{\mathrm{S} 7}$ We now report results using the protocol adopted in this paper for consistency.

\section{[1-cyclohexane][BAr ${ }_{4}$ ] (Partially Optimised)}

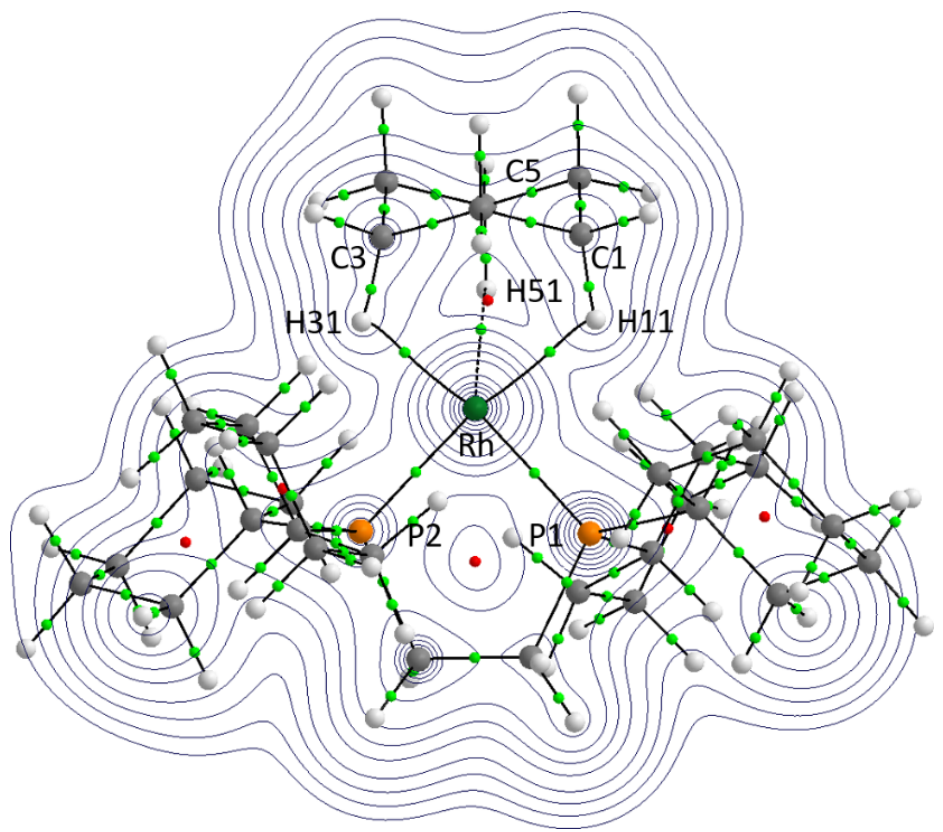

Figure S97: QTAIM molecular graph of [1-cyclohexane][BAr ${ }_{4}$ ] (partially optimised) with electron density contours in the H11-Rh-H31 plane.
Table S21: QTAIM BCP data and bond lengths for key bonds.

\begin{tabular}{ccccc}
\hline $\begin{array}{c}\text { Bond } \\
\text { Path }\end{array}$ & $\begin{array}{c}\text { Interatomic } \\
\text { Distance } \\
(\mathbf{A})\end{array}$ & $\begin{array}{c}\boldsymbol{\rho} \\
\left(\mathbf{e} \mathbf{b o h r}^{-3}\right)\end{array}$ & $\begin{array}{c}\boldsymbol{\nabla}^{2} \mathbf{\rho} \\
(\mathbf{e} \text { bohr }\end{array}$ & $\mathbf{H} \mathbf{( r )}$ \\
\hline C1-H11 & 1.137 & 0.2415 & -0.6706 & -0.2186 \\
H11-Rh & 1.933 & 0.0503 & 0.1815 & -0.0079 \\
C1-Rh & 2.642 & & No BCP & \\
Rh-P1 & 2.192 & 0.1228 & 0.0703 & -0.0630 \\
C3-H31 & 1.141 & 0.2388 & -0.6476 & -0.2139 \\
H31-Rh & 1.852 & 0.0610 & 0.2257 & -0.0114 \\
C3-Rh & 2.530 & & No BCP & \\
Rh-P2 & 2.192 & 0.1226 & 0.0647 & -0.0633 \\
C5-H51 & 1.109 & 0.2660 & -0.8574 & -0.2599 \\
H51-Rh & 2.677 & 0.0148 & 0.0386 & 0.0000 \\
C5-Rh & 3.306 & & No BCP & \\
\hline
\end{tabular}




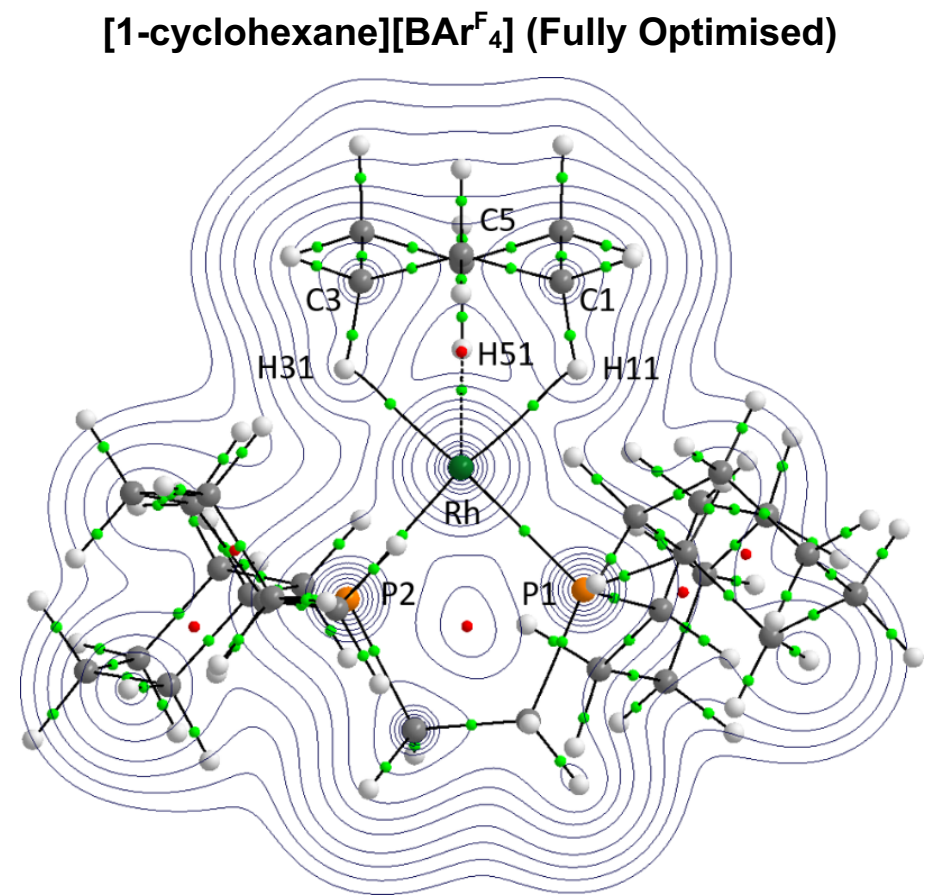

Figure S98: QTAIM molecular graph of [1-cyclohexane][BAr ${ }_{4}$ ] (fully optimised) with electron density contours in the H11-Rh-H31 plane.
Table S22: QTAIM BCP data and bond lengths for key bonds.

\begin{tabular}{ccccc}
\hline $\begin{array}{c}\text { Bond } \\
\text { Path }\end{array}$ & $\begin{array}{c}\text { Interatomic } \\
\text { Distance } \\
(\mathbf{A})\end{array}$ & $\begin{array}{c}\boldsymbol{\rho} \\
\left(\mathbf{e} \mathbf{b o h r}^{-3}\right)\end{array}$ & $\begin{array}{c}\boldsymbol{\nabla}^{2} \mathbf{\rho} \\
(\mathbf{e} \text { bohr }\end{array}$ & $\mathbf{H})$ \\
\hline C1-H11 & 1.139 & 0.2392 & -0.6538 & -0.2148 \\
H11-Rh & 1.923 & 0.0505 & 0.1815 & -0.0082 \\
C1-Rh & 2.681 & & No BCP & \\
Rh-P1 & 2.195 & 0.1216 & 0.0657 & -0.0621 \\
C3-H31 & 1.140 & 0.2388 & -0.6514 & -0.2141 \\
H31-Rh & 1.928 & 0.0495 & 0.1858 & -0.0074 \\
C3-Rh & 2.679 & & No BCP & \\
Rh-P2 & 2.201 & 0.1202 & 0.0712 & -0.0604 \\
C5-H51 & 1.109 & 0.2660 & -0.8574 & -0.2599 \\
H51-Rh & 2.677 & 0.0148 & 0.0386 & 0.0000 \\
C5-Rh & 3.306 & & No BCP & \\
\hline
\end{tabular}



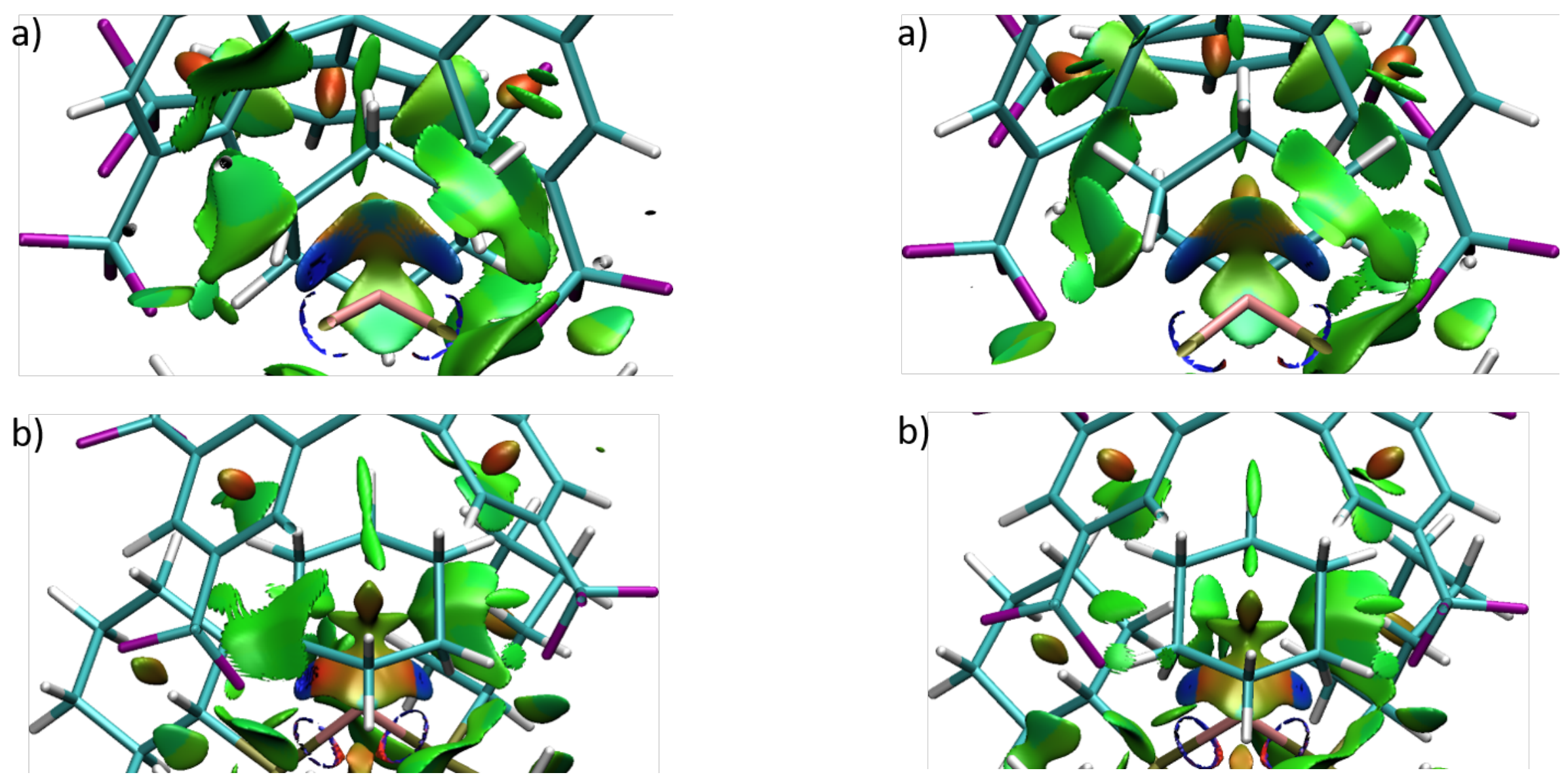

Figure S99: a) NCl Plot of [1-cyclohexane][BAr ${ }_{4}$ ] (partially optimised) with Figure S100: a) NCl Plot of [1-cyclohexane][BAr ${ }_{4}$ ] (fully optimised) with isosurfaces generated for $s=0.3$ au and $-0.07<\rho<0.07$ au. View from Rh isosurfaces generated for $s=0.3$ au and $-0.07<\rho<0.07$ au. View from Rh looking towards the alkane. b) View from above the alkane looking towards looking towards the alkane. b) View from above the alkane looking towards Rh. $\mathrm{Rh}$. 
Table S23: NBO data from [1-cyclohexane][BAr ${ }_{4}$ ] (partially optimised) showing the donating and accepting orbitals occupation and the $2^{\text {nd }}$ order perturbation theory interaction energy.

\begin{tabular}{ccccc}
\hline $\begin{array}{c}\text { Donating } \\
\text { Orbital }\end{array}$ & Occupation & $\begin{array}{c}\text { Accepting } \\
\text { Orbital }\end{array}$ & Occupation & $\begin{array}{c}\text { Energy } \\
\text { (kcal/mol) }\end{array}$ \\
\hline \multicolumn{5}{c}{ C3-H31 } \\
\hline$\sigma(\mathrm{C}-\mathrm{H})$ & 1.89 & $\sigma^{*}(\mathrm{Rh}-$ & 0.28 & 15.13 \\
& & $\left.\mathrm{P}_{\text {trans }}\right)$ & & \\
LP $(\mathrm{Rh})$ & 1.97 & $\sigma^{*}(\mathrm{C}-\mathrm{H})$ & 0.03 & 0.14 \\
LP $(\mathrm{Rh})$ & 1.90 & $\sigma^{*}(\mathrm{C}-\mathrm{H})$ & 0.03 & 0.50 \\
LP $(\mathrm{Rh})$ & 1.88 & $\sigma^{*}(\mathrm{C}-\mathrm{H})$ & 0.03 & 2.60 \\
$\sigma\left(\mathrm{Rh}-\mathrm{P}_{\text {cis }}\right)$ & 1.81 & $\sigma^{*}(\mathrm{C}-\mathrm{H})$ & 0.03 & 5.45 \\
$\sigma(\mathrm{Rh}-$ & 1.81 & $\sigma^{*}(\mathrm{C}-\mathrm{H})$ & 0.03 & 1.76 \\
$\left.\mathrm{P}_{\text {trans }}\right)$ & & & & \\
\hline
\end{tabular}

\begin{tabular}{ccccc}
\multicolumn{5}{c}{$\mathrm{C} 1-\mathrm{H} 11$} \\
\hline$\sigma(\mathrm{C}-\mathrm{H})$ & 1.88 & $\sigma^{*}(\mathrm{Rh}-$ & 0.29 & 20.03 \\
& & $\left.\mathrm{P}_{\text {trans }}\right)$ & & \\
LP $(\mathrm{Rh})$ & 1.97 & $\sigma^{*}(\mathrm{C}-\mathrm{H})$ & 0.04 & 0.08 \\
LP (Rh) & 1.95 & $\sigma^{*}(\mathrm{C}-\mathrm{H})$ & 0.04 & 0.42 \\
LP (Rh) & 1.90 & $\sigma^{*}(\mathrm{C}-\mathrm{H})$ & 0.04 & 0.23 \\
LP (Rh) & 1.88 & $\sigma^{*}(\mathrm{C}-\mathrm{H})$ & 0.04 & 4.26 \\
$\sigma\left(\mathrm{Rh}-\mathrm{P}_{\text {cis }}\right)$ & 1.81 & $\sigma^{*}(\mathrm{C}-\mathrm{H})$ & 0.04 & 5.99
\end{tabular}

\begin{tabular}{ccccc}
$\begin{array}{c}\sigma(\mathrm{Rh}- \\
\left.\mathrm{P}_{\text {trans }}\right)\end{array}$ & 1.81 & $\sigma^{*}(\mathrm{C}-\mathrm{H})$ & 0.04 & 1.63 \\
\hline & & & \\
\hline$\sigma(\mathrm{C}-\mathrm{H})$ & 1.97 & $\sigma^{*}(\mathrm{Rh}-\mathrm{P})$ & 0.28 & 1.01 \\
$\sigma(\mathrm{C}-\mathrm{H})$ & 1.97 & $\sigma^{*}(\mathrm{Rh}-\mathrm{P})$ & 0.29 & 0.68 \\
LP (Rh) & 1.97 & $\sigma^{*}(\mathrm{C}-\mathrm{H})$ & 0.02 & 0.78 \\
LP (Rh) & 1.90 & $\sigma^{*}(\mathrm{C}-\mathrm{H})$ & 0.02 & 0.46 \\
LP (Rh) & 1.88 & $\sigma^{*}(\mathrm{C}-\mathrm{H})$ & 0.02 & 0.07 \\
$\sigma(\mathrm{Rh}-\mathrm{P})$ & 1.81 & $\sigma^{*}(\mathrm{C}-\mathrm{H})$ & 0.02 & 0.89 \\
$\sigma(\mathrm{Rh}-\mathrm{P})$ & 1.81 & $\sigma^{*}(\mathrm{C}-\mathrm{H})$ & 0.02 & 0.95 \\
\hline
\end{tabular}


Table S24: NBO data from [1-cyclohexane][BAr ${ }_{4}$ ] (fully optimised) showing the donating and accepting orbitals occupation and the $2^{\text {nd }}$ order perturbation theory interaction energy. Selected donor-acceptor interactions are shown below.

\begin{tabular}{|c|c|c|c|c|}
\hline $\begin{array}{l}\text { Donating } \\
\text { Orbital }\end{array}$ & Occupation & $\begin{array}{c}\text { Accepting } \\
\text { Orbital }\end{array}$ & Occupation & $\begin{array}{c}\text { Energy } \\
\text { (kcal/mol) }\end{array}$ \\
\hline \multicolumn{5}{|c|}{ C3-H31 } \\
\hline$\sigma(\mathrm{C}-\mathrm{H})$ & 1.89 & $\begin{array}{c}\sigma^{*}(\mathrm{Rh}- \\
\left.\mathrm{P}_{\text {trans }}\right)\end{array}$ & 0.28 & 15.81 \\
\hline LP (Rh) & 1.97 & $\sigma^{*}(\mathrm{C}-\mathrm{H})$ & 0.04 & 0.14 \\
\hline LP (Rh) & 1.90 & $\sigma^{*}(\mathrm{C}-\mathrm{H})$ & 0.04 & 0.21 \\
\hline LP (Rh) & 1.89 & $\sigma^{*}(\mathrm{C}-\mathrm{H})$ & 0.04 & $2.72^{\mathrm{a}}$ \\
\hline$\sigma\left(\mathrm{Rh}-\mathrm{P}_{\text {cis }}\right)$ & 1.81 & $\sigma^{*}(\mathrm{C}-\mathrm{H})$ & 0.04 & $5.31^{b}$ \\
\hline $\begin{array}{c}\sigma(\mathrm{Rh}- \\
\left.\mathrm{P}_{\text {trans }}\right)\end{array}$ & 1.82 & $\sigma^{*}(\mathrm{C}-\mathrm{H})$ & 0.04 & 1.88 \\
\hline \multicolumn{5}{|c|}{$\mathrm{C} 1-\mathrm{H} 11$} \\
\hline$\sigma(\mathrm{C}-\mathrm{H})$ & 1.89 & $\begin{array}{c}\sigma^{*}(\mathrm{Rh}- \\
\left.\mathrm{P}_{\text {trans }}\right)\end{array}$ & 0.28 & 16.51 \\
\hline$\sigma(\mathrm{C}-\mathrm{H})$ & 1.89 & $\sigma^{*}\left(\mathrm{Rh}-\mathrm{P}_{\text {cis }}\right)$ & 0.28 & 0.10 \\
\hline LP (Rh) & 1.97 & $\sigma^{*}(\mathrm{C}-\mathrm{H})$ & 0.03 & 0.08 \\
\hline LP (Rh) & 1.95 & $\sigma^{*}(\mathrm{C}-\mathrm{H})$ & 0.03 & 0.08 \\
\hline LP (Rh) & 1.90 & $\sigma^{*}(\mathrm{C}-\mathrm{H})$ & 0.03 & 0.10 \\
\hline $\mathrm{LP}(\mathrm{Rh})$ & 1.89 & $\sigma^{*}(\mathrm{C}-\mathrm{H})$ & 0.03 & 2.30 \\
\hline
\end{tabular}

\begin{tabular}{ccccc}
$\sigma\left(\mathrm{Rh}-\mathrm{P}_{\text {cis }}\right)$ & 1.82 & $\sigma^{*}(\mathrm{C}-\mathrm{H})$ & 0.03 & 5.54 \\
$\sigma(\mathrm{Rh}-$ & 1.81 & $\sigma^{*}(\mathrm{C}-\mathrm{H})$ & 0.03 & 2.30 \\
$\left.\mathrm{P}_{\text {trans }}\right)$ & & & \\
\hline \multicolumn{5}{c}{$\mathrm{C} 5-\mathrm{H} 51$} \\
\hline$\sigma(\mathrm{C}-\mathrm{H})$ & 1.97 & $\sigma^{*}(\mathrm{Rh}-\mathrm{P})$ & 0.28 & 0.81 \\
$\sigma(\mathrm{C}-\mathrm{H})$ & 1.97 & $\sigma^{*}(\mathrm{Rh}-\mathrm{P})$ & 0.28 & 0.69 \\
LP (Rh) & 1.97 & $\sigma^{*}(\mathrm{C}-\mathrm{H})$ & 0.02 & 1.01 \\
$\mathrm{LP}(\mathrm{Rh})$ & 1.90 & $\sigma^{*}(\mathrm{C}-\mathrm{H})$ & 0.02 & 0.48 \\
$\sigma(\mathrm{Rh}-\mathrm{P})$ & 1.82 & $\sigma^{*}(\mathrm{C}-\mathrm{H})$ & 0.02 & 0.89 \\
$\sigma(\mathrm{Rh}-\mathrm{P})$ & 1.81 & $\sigma^{*}(\mathrm{C}-\mathrm{H})$ & 0.02 & 0.90 \\
\hline
\end{tabular}

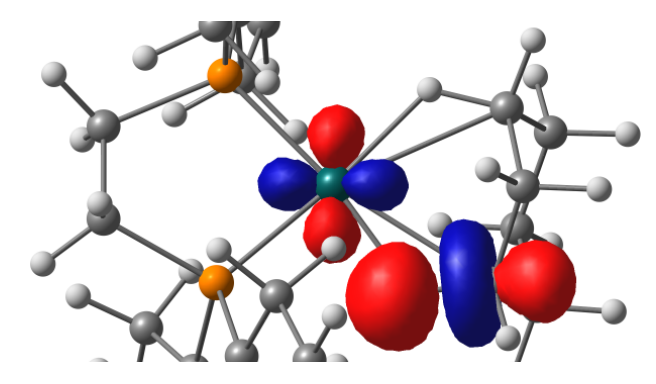

$\mathrm{a}=\Delta \mathrm{E}=2.72 \mathrm{kcal} / \mathrm{mol}$

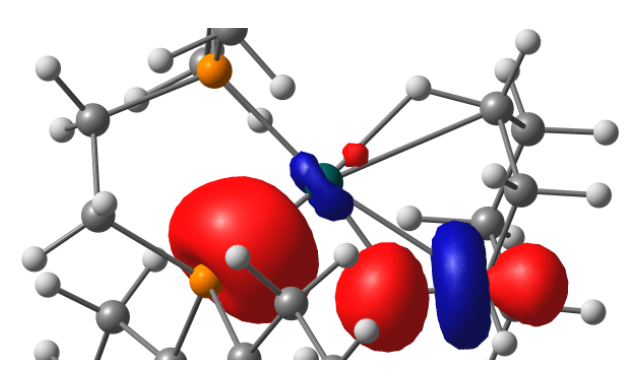

$\mathrm{b}=\Delta \mathrm{E}=5.31 \mathrm{kcal} / \mathrm{mol}$ 

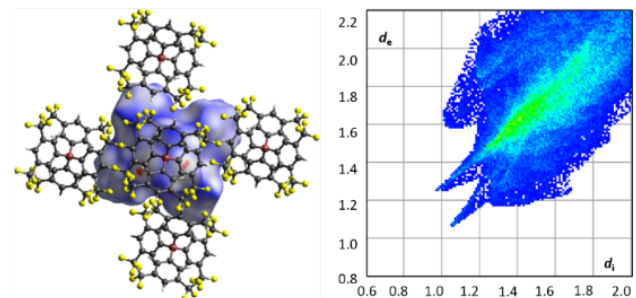

Full System
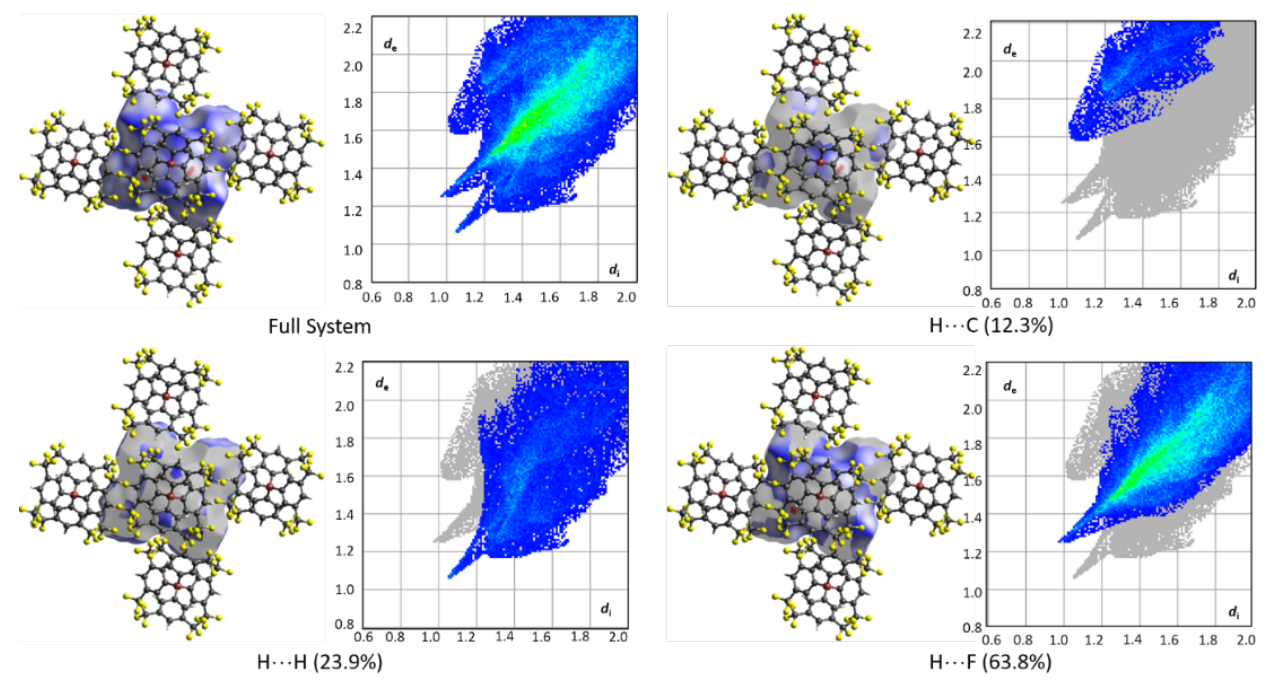

Figure S101: Hirshfeld Surfaces of [1-cyclohexane][BAr $\left.{ }_{4}\right]$ (partially optimised) showing normalised distance and associated fingerprint plots. Percentage of each type of contact is shown in brackets.
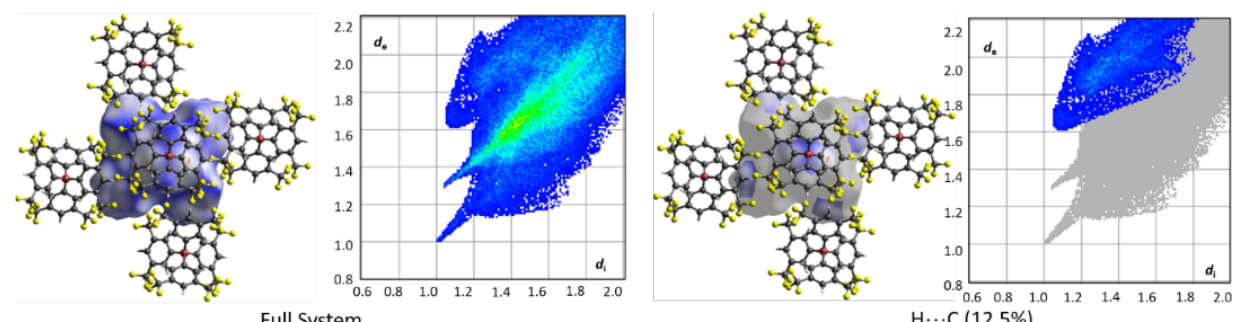

Full System
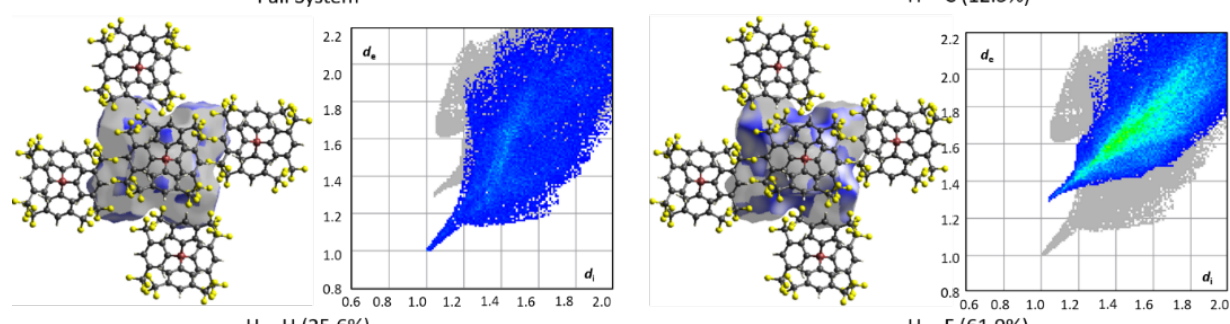

$\mathrm{H} \cdots \mathrm{F}(61.9 \%)$

Figure S102: Hirshfeld Surfaces of [1-cyclohexane][BAr${ }_{4}$ ] (fully optimised) showing normalised distance and associated fingerprint plots. Percentage of each type of contact is shown in brackets. 


\section{S.5.7. $[1-\mathrm{NBA}]\left[\mathrm{BAr}_{4}^{\mathrm{F}}\right]$}

Note: The structure of [1-NBA][BAr$\left.{ }_{4}\right]$ has been previous reported. ${ }^{\mathrm{S2}}$ We now report results using the protocol adopted in this paper for consistency.

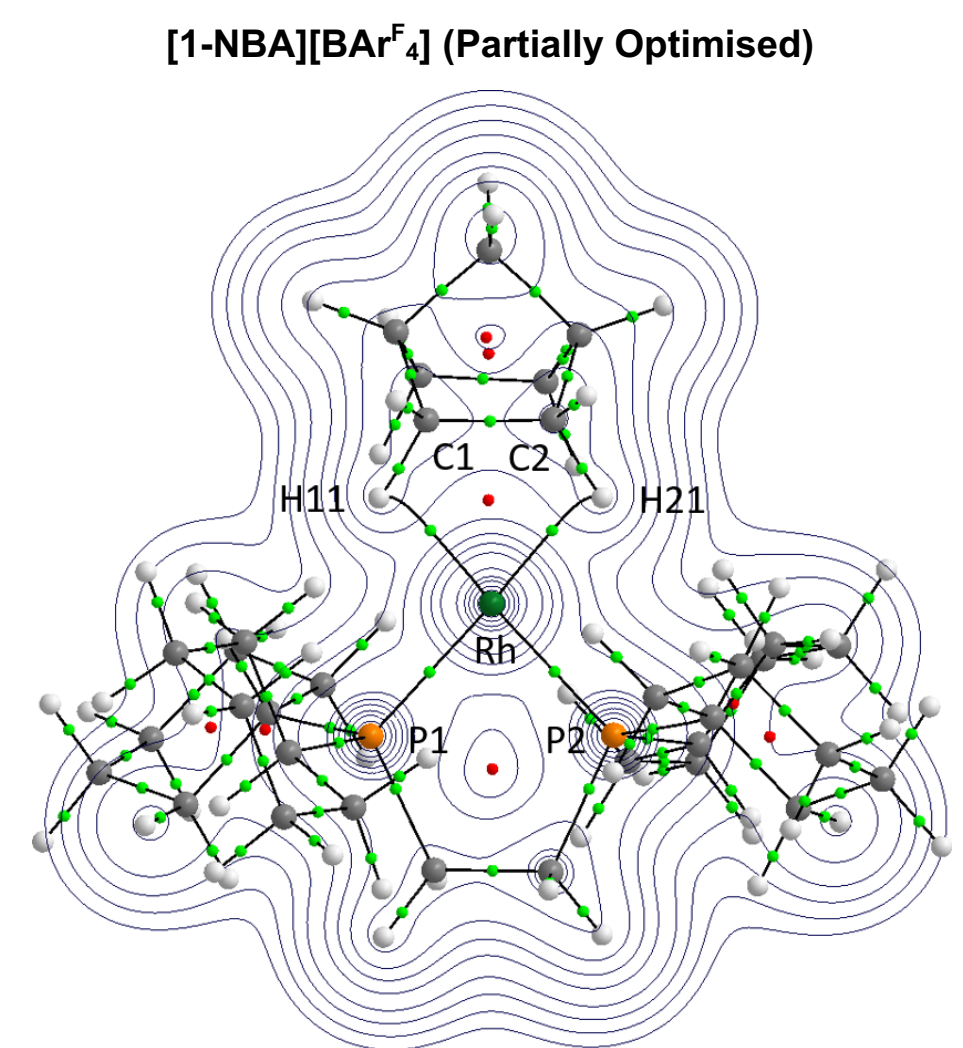

Table S25: QTAIM BCP data and bond lengths for key bonds.

\begin{tabular}{ccccc}
\hline $\begin{array}{c}\text { Bond } \\
\text { Path }\end{array}$ & $\begin{array}{c}\text { Distance } \\
(\AA)\end{array}$ & $\begin{array}{c}\rho \\
\left(\mathbf{e ~ b o h r} \mathbf{r}^{-3}\right)\end{array}$ & $\begin{array}{c}\boldsymbol{\nabla}^{2} \boldsymbol{\rho} \\
(\mathbf{e} \text { bohr }\end{array}$ & $\mathbf{H}(\mathbf{r})$ \\
\hline C1-H11 & 1.153 & 0.2337 & -0.6206 & -0.2040 \\
H11-Rh & 1.889 & 0.0593 & 0.2463 & -0.0075 \\
C1-Rh & 2.400 & & No BCP & \\
Rh-P1 & 2.195 & 0.1218 & 0.0733 & -0.0619 \\
C2 -H21 & 1.155 & 0.2321 & -0.6088 & -0.2012 \\
H21-Rh & 1.872 & 0.0617 & 0.2534 & -0.0086 \\
C2-Rh & 2.389 & & No BCP & \\
Rh-P2 & 2.193 & 0.1221 & 0.0710 & -0.0624 \\
\hline
\end{tabular}

Figure S103: QTAIM molecular graph of [1-NBA] BAr $_{4}{ }_{4}$ ] (partially optimised) with electron density contours in the $\mathrm{H} 11-\mathrm{Rh}-\mathrm{H} 21$ plane. 
[1-NBA][BAr $\left.{ }_{4}\right]$ (Fully Optimised)

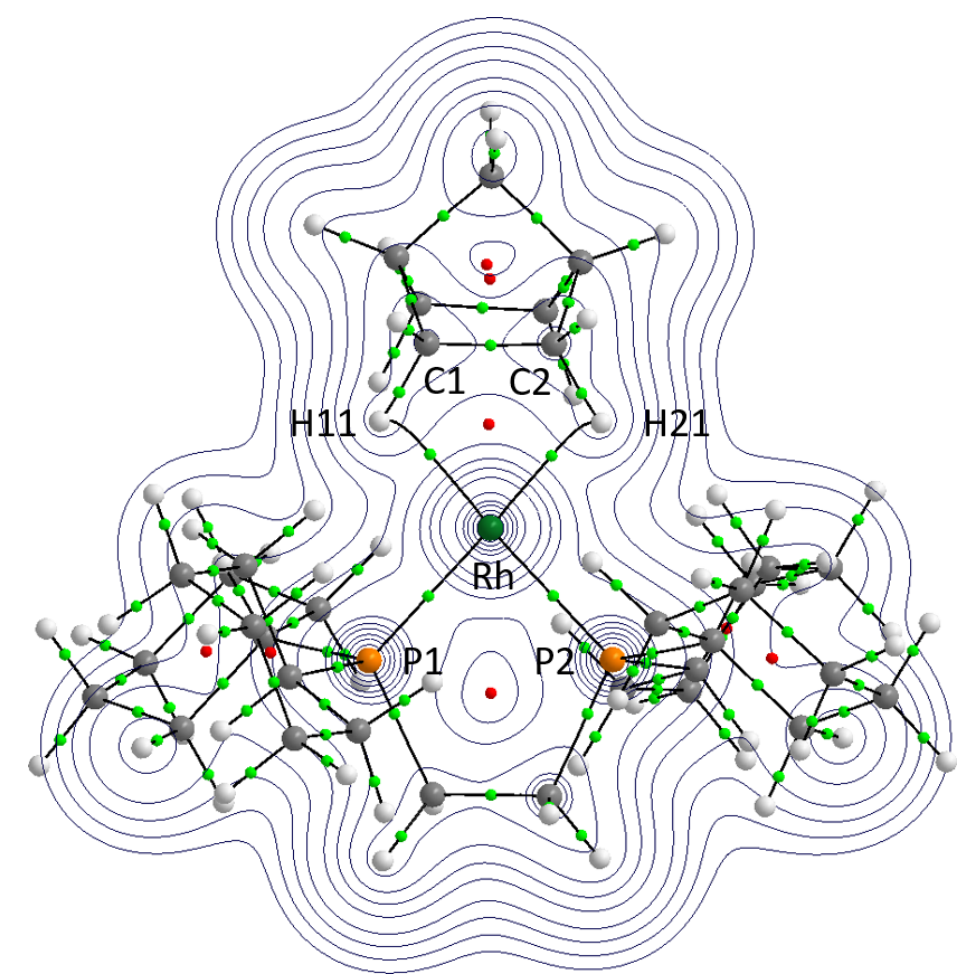

Table S26: QTAIM BCP data and bond lengths for key bonds.

\begin{tabular}{|c|c|c|c|c|}
\hline \multirow[b]{2}{*}{$\begin{array}{l}\text { Bond } \\
\text { Path }\end{array}$} & \multicolumn{4}{|c|}{ Interatomic } \\
\hline & $\begin{array}{c}\text { Distance } \\
(\AA)\end{array}$ & $\begin{array}{c}\rho \\
\left(e^{-} b o h r^{-3}\right)\end{array}$ & $\begin{array}{c}\nabla^{2} \rho \\
\left(e-b o h r^{-5}\right)\end{array}$ & $H(r)$ \\
\hline $\mathrm{C} 1-\mathrm{H} 11$ & 1.153 & 0.2336 & -0.6209 & -0.2038 \\
\hline $\mathrm{H} 11-\mathrm{Rh}$ & 1.892 & 0.0584 & 0.2412 & -0.0073 \\
\hline C1-Rh & 2.415 & & No BCP & \\
\hline Rh-P1 & 2.210 & 0.1186 & 0.0788 & -0.0582 \\
\hline $\mathrm{C} 2-\mathrm{H} 21$ & 1.154 & 0.2325 & -0.6134 & -0.2022 \\
\hline $\mathrm{H} 21-\mathrm{Rh}$ & 1.877 & 0.0604 & 0.2474 & -0.0083 \\
\hline C2-Rh & 2.406 & & No BCP & \\
\hline Rh-P2 & 2.210 & 0.1185 & 0.0774 & -0.0583 \\
\hline
\end{tabular}

Figure S104: QTAIM molecular graph of [1-NBA][BAr ${ }_{4}$ ] (fully optimised) with electron density contours in the $\mathrm{H} 11-\mathrm{Rh}-\mathrm{H} 21$ plane. 
a)

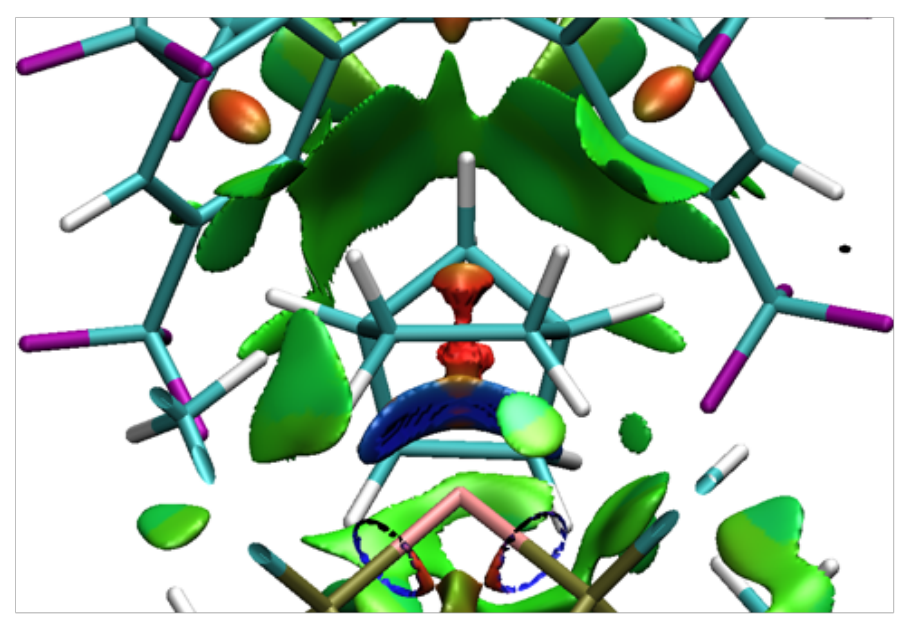

b)

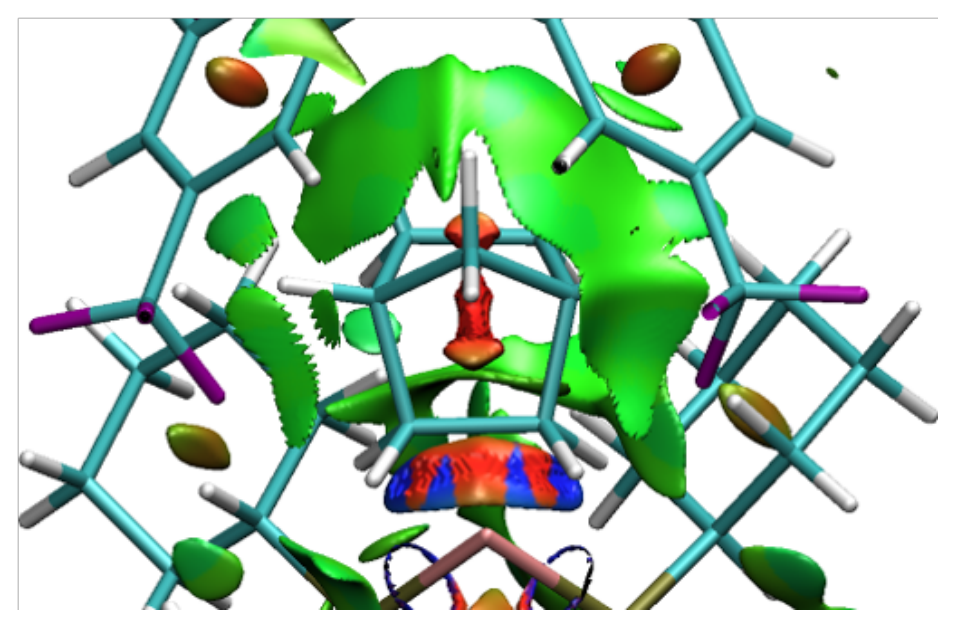

a)

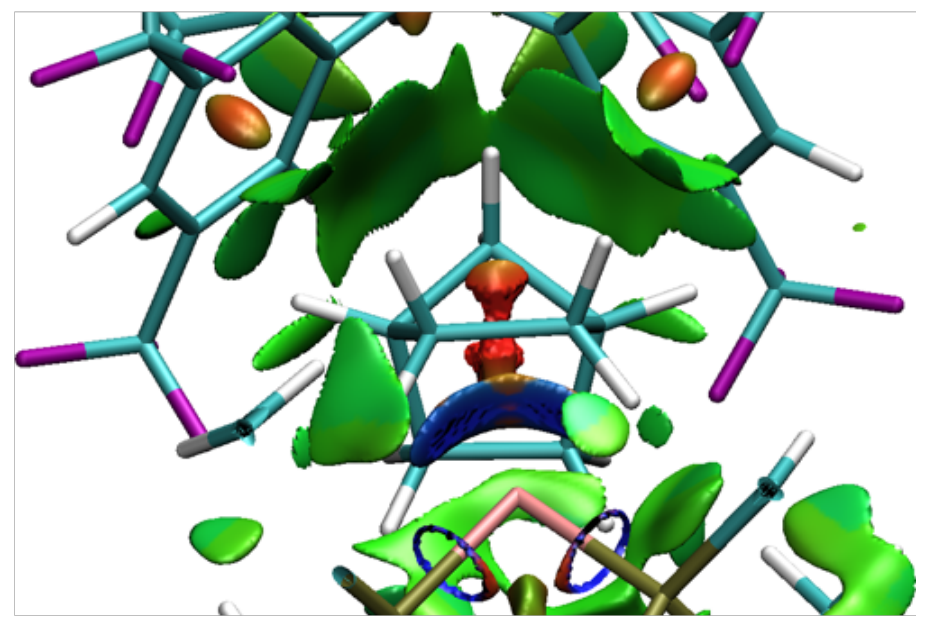

b)

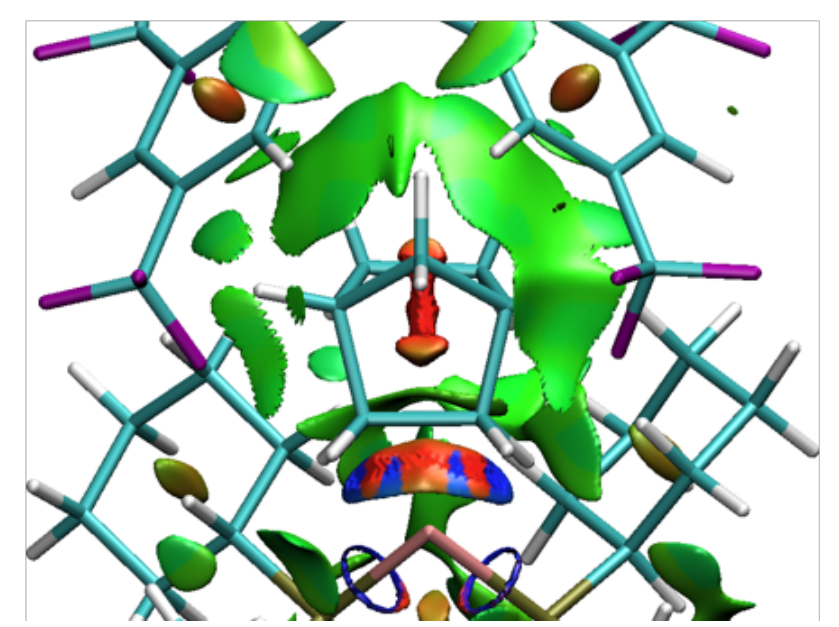

Figure S105: a) NCl Plot of [1-NBA][BAr ${ }_{4}$ ] (partially optimised) with Figure S106: a) NCl Plot of [1-NBA][BAr ${ }_{4}$ ] (fully optimised) with isosurfaces isosurfaces generated for $s=0.3$ au and $-0.07<\rho<0.07$ au. View from Rh generated for $s=0.3$ au and $-0.07<\rho<0.07$ au. View from Rh looking looking towards the alkane. b) View from above the alkane looking towards towards the alkane. b) View from above the alkane looking towards Rh. Rh. 
Table S27: NBO data from [1-NBA][BAr ${ }_{4}$ ] (partially optimised) showing the donating and accepting orbitals occupation and the $2^{\text {nd }}$ order perturbation theory interaction energy.

\begin{tabular}{|c|c|c|c|c|}
\hline $\begin{array}{c}\text { Donating } \\
\text { Orbital }\end{array}$ & Occupation & $\begin{array}{c}\text { Accepting } \\
\text { Orbital }\end{array}$ & Occupation & $\begin{array}{c}\text { Energy } \\
\text { (kcal/mol) }\end{array}$ \\
\hline \multicolumn{5}{|c|}{$\mathrm{C} 2-\mathrm{H} 21$} \\
\hline$\sigma(\mathrm{C}-\mathrm{H})$ & 1.87 & $\begin{array}{c}\sigma^{*}(\mathrm{Rh}- \\
\left.\mathrm{P}_{\text {trans }}\right)\end{array}$ & 0.29 & 20.83 \\
\hline$\sigma(\mathrm{C}-\mathrm{H})$ & 1.87 & $\sigma^{*}\left(\mathrm{Rh}-\mathrm{P}_{\text {cis }}\right)$ & 0.29 & 0.10 \\
\hline LP (Rh) & 1.97 & $\sigma^{*}(\mathrm{C}-\mathrm{H})$ & 0.04 & 0.05 \\
\hline LP (Rh) & 1.95 & $\sigma^{*}(\mathrm{C}-\mathrm{H})$ & 0.04 & 0.25 \\
\hline LP (Rh) & 1.87 & $\sigma^{*}(\mathrm{C}-\mathrm{H})$ & 0.04 & 4.15 \\
\hline$\sigma\left(\mathrm{Rh}-\mathrm{P}_{\text {cis }}\right)$ & 1.83 & $\sigma^{*}(\mathrm{C}-\mathrm{H})$ & 0.04 & 3.73 \\
\hline $\begin{array}{l}\sigma(\mathrm{Rh}- \\
\left.\mathrm{P}_{\text {trans }}\right)\end{array}$ & 1.83 & $\sigma^{*}(\mathrm{C}-\mathrm{H})$ & 0.04 & 1.18 \\
\hline \multicolumn{5}{|c|}{$\mathrm{C} 1-\mathrm{H} 11$} \\
\hline$\sigma(\mathrm{C}-\mathrm{H})$ & 1.87 & $\begin{array}{c}\sigma^{*}(\mathrm{Rh}- \\
\left.P_{\text {trans }}\right)\end{array}$ & 0.29 & 22.35 \\
\hline$\sigma(\mathrm{C}-\mathrm{H})$ & 1.87 & $\sigma^{*}\left(\mathrm{Rh}-\mathrm{P}_{\text {cis }}\right)$ & 0.29 & 0.14 \\
\hline LP (Rh) & 1.95 & $\sigma^{*}(\mathrm{C}-\mathrm{H})$ & 0.04 & 0.12 \\
\hline LP (Rh) & 1.87 & $\sigma^{*}(\mathrm{C}-\mathrm{H})$ & 0.04 & 4.65 \\
\hline$\sigma\left(\mathrm{Rh}-\mathrm{P}_{\text {cis }}\right)$ & 1.83 & $\sigma^{*}(\mathrm{C}-\mathrm{H})$ & 0.04 & 3.62 \\
\hline $\begin{array}{c}\sigma(\mathrm{Rh}- \\
\left.\mathrm{P}_{\text {trans }}\right)\end{array}$ & 1.83 & $\sigma^{*}(\mathrm{C}-\mathrm{H})$ & 0.04 & 1.07 \\
\hline
\end{tabular}

Table S28: NBO data from [1-NBA][BAr ${ }_{4}$ ] (fully optimised) showing the donating and accepting orbitals occupation and the $2^{\text {nd }}$ order perturbation theory interaction energy.

\begin{tabular}{|c|c|c|c|c|}
\hline $\begin{array}{c}\text { Donating } \\
\text { Orbital }\end{array}$ & Occupation & $\begin{array}{c}\text { Accepting } \\
\text { Orbital }\end{array}$ & Occupation & $\begin{array}{c}\text { Energy } \\
\text { (kcal/mol) }\end{array}$ \\
\hline \multicolumn{5}{|c|}{$\mathrm{C} 2-\mathrm{H} 21$} \\
\hline$\sigma(\mathrm{C}-\mathrm{H})$ & 1.87 & $\begin{array}{c}\sigma^{*}(\mathrm{Rh}- \\
\left.P_{\text {trans }}\right)\end{array}$ & 0.28 & 20.46 \\
\hline$\sigma(\mathrm{C}-\mathrm{H})$ & 1.87 & $\sigma^{*}\left(\mathrm{Rh}-\mathrm{P}_{\text {cis }}\right)$ & 0.29 & 0.08 \\
\hline LP (Rh) & 1.97 & $\sigma^{*}(\mathrm{C}-\mathrm{H})$ & 0.04 & 0.06 \\
\hline LP (Rh) & 1.95 & $\sigma^{*}(\mathrm{C}-\mathrm{H})$ & 0.04 & 0.25 \\
\hline $\mathrm{LP}(\mathrm{Rh})$ & 1.87 & $\sigma^{*}(\mathrm{C}-\mathrm{H})$ & 0.04 & 3.99 \\
\hline$\sigma\left(\mathrm{Rh}-\mathrm{P}_{\text {cis }}\right)$ & 1.83 & $\sigma^{*}(\mathrm{C}-\mathrm{H})$ & 0.04 & 3.53 \\
\hline $\begin{array}{c}\sigma(\mathrm{Rh}- \\
\left.\mathrm{P}_{\text {trans }}\right)\end{array}$ & 1.83 & $\sigma^{*}(\mathrm{C}-\mathrm{H})$ & 0.04 & 1.16 \\
\hline \multicolumn{5}{|c|}{$\mathrm{C} 1-\mathrm{H} 11$} \\
\hline$\sigma(\mathrm{C}-\mathrm{H})$ & 1.87 & $\begin{array}{c}\sigma^{*}(\mathrm{Rh}- \\
\left.\mathrm{P}_{\text {trans }}\right)\end{array}$ & 0.29 & 21.82 \\
\hline$\sigma(\mathrm{C}-\mathrm{H})$ & 1.87 & $\sigma^{*}\left(\mathrm{Rh}-\mathrm{P}_{\text {cis }}\right)$ & 0.28 & 0.10 \\
\hline LP (Rh) & 1.97 & $\sigma^{*}(\mathrm{C}-\mathrm{H})$ & 0.04 & 0.06 \\
\hline LP (Rh) & 1.95 & $\sigma^{*}(\mathrm{C}-\mathrm{H})$ & 0.04 & 0.07 \\
\hline LP (Rh) & 1.87 & $\sigma^{*}(\mathrm{C}-\mathrm{H})$ & 0.04 & 4.53 \\
\hline$\sigma\left(\mathrm{Rh}-\mathrm{P}_{\text {cis }}\right)$ & 1.83 & $\sigma^{*}(\mathrm{C}-\mathrm{H})$ & 0.04 & 3.49 \\
\hline $\begin{array}{c}\sigma(\mathrm{Rh}- \\
\left.\mathrm{P}_{\text {trans }}\right)\end{array}$ & 1.83 & $\sigma^{*}(\mathrm{C}-\mathrm{H})$ & 0.04 & 1.05 \\
\hline
\end{tabular}




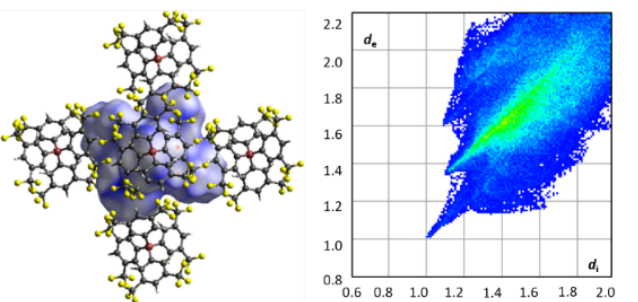

Full System
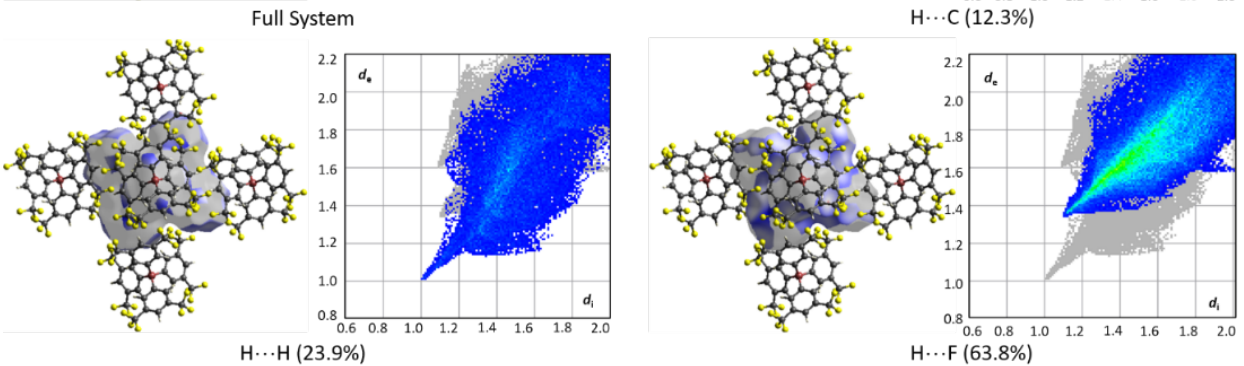

Figure S107: Hirshfeld Surfaces of [1-NBA][BAr ${ }_{4}$ ] (partially optimised) showing normalised distance and associated fingerprint plots. Percentage of each type of contact is shown in brackets.
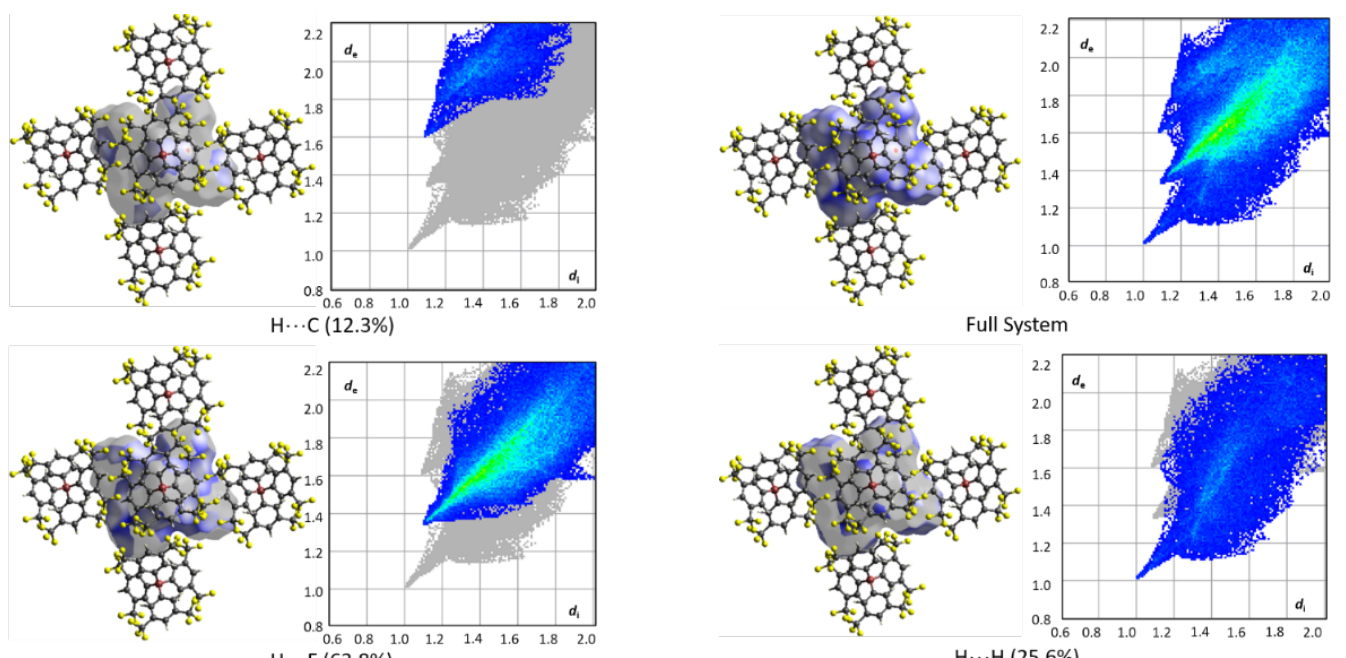

$H \cdots H(25.6 \%)$

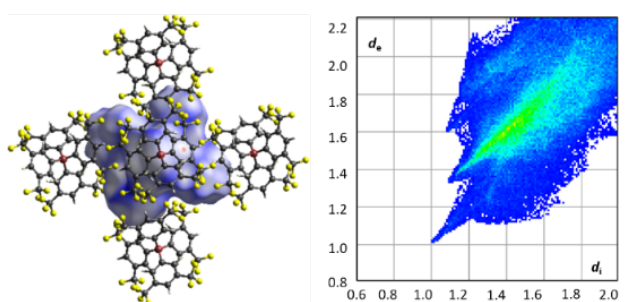

Figure S108: Hirshfeld Surfaces of [1-NBA][BAr ${ }_{4}$ ] (fully optimised) showing normalised distance and associated fingerprint plots. Percentage of each type of contact is shown in brackets.
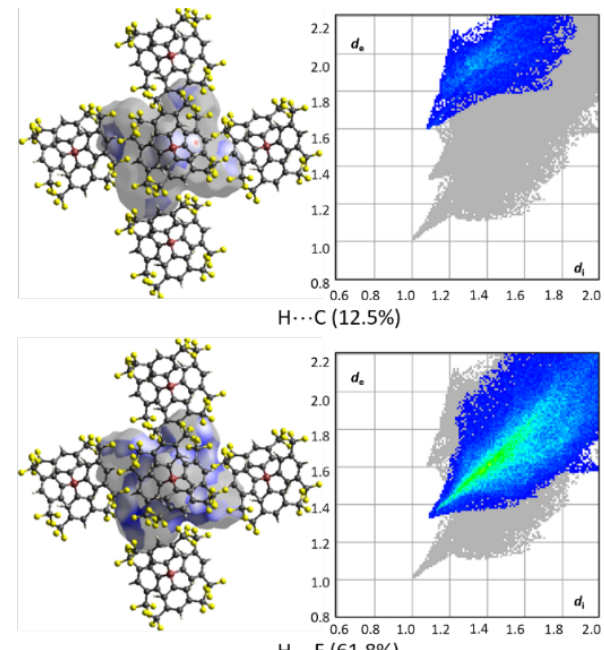

$\mathrm{H} \cdots \mathrm{F}(61.8 \%)$ 


\section{S.5.8. [1-isobutane][BAr $\left.{ }_{4}\right]$}

Note: The structure of [1-isobutane][BAr ${ }_{4}$ ] has been previous reported. ${ }^{57}$ We now report results using the protocol adopted in this paper for consistency.

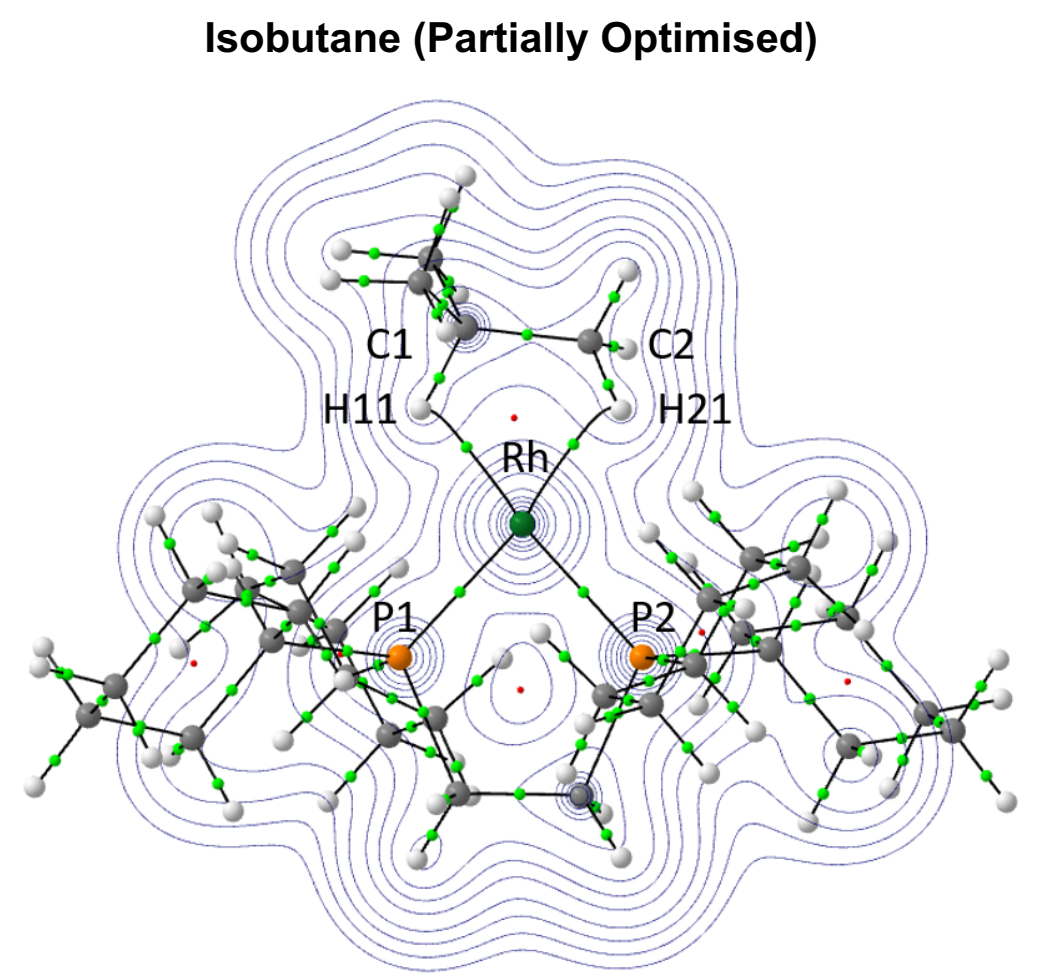

Figure S109: QTAIM molecular graph of [1-isobutane][BAr ${ }_{4}$ ] (partially optimised) with electron density contours in the H11-RhH21 plane.
Table S29: QTAIM BCP data and bond lengths for key bonds.

\begin{tabular}{|c|c|c|c|c|}
\hline & Interatomic & & & \\
\hline $\begin{array}{l}\text { Bond } \\
\text { Path }\end{array}$ & $\begin{array}{c}\text { Distance } \\
(\AA)\end{array}$ & $\begin{array}{c}\rho \\
\left(e b^{-3} r^{-3}\right)\end{array}$ & $\begin{array}{c}\nabla^{2} \rho \\
\left(e b^{2} b r^{-5}\right)\end{array}$ & $H(r)$ \\
\hline $\mathrm{C} 1-\mathrm{H} 11$ & 1.160 & 0.2316 & -0.6036 & -0.1997 \\
\hline $\mathrm{H} 11-\mathrm{Rh}$ & 1.873 & 0.0603 & 0.2414 & -0.0090 \\
\hline C1-Rh & 2.442 & & No BCP & \\
\hline Rh-P1 & 2.191 & 0.1127 & 0.0679 & -0.0631 \\
\hline $\mathrm{C} 2-\mathrm{H} 21$ & 1.148 & 0.2364 & -0.6433 & -0.2093 \\
\hline $\mathrm{H} 21-\mathrm{Rh}$ & 1.933 & 0.0562 & 0.2467 & -0.0042 \\
\hline C2-Rh & 2.363 & & No BCP & \\
\hline Rh-P2 & 2.183 & 0.1243 & 0.0658 & -0.0650 \\
\hline
\end{tabular}




\section{Isobutane (Fully Optimised)}

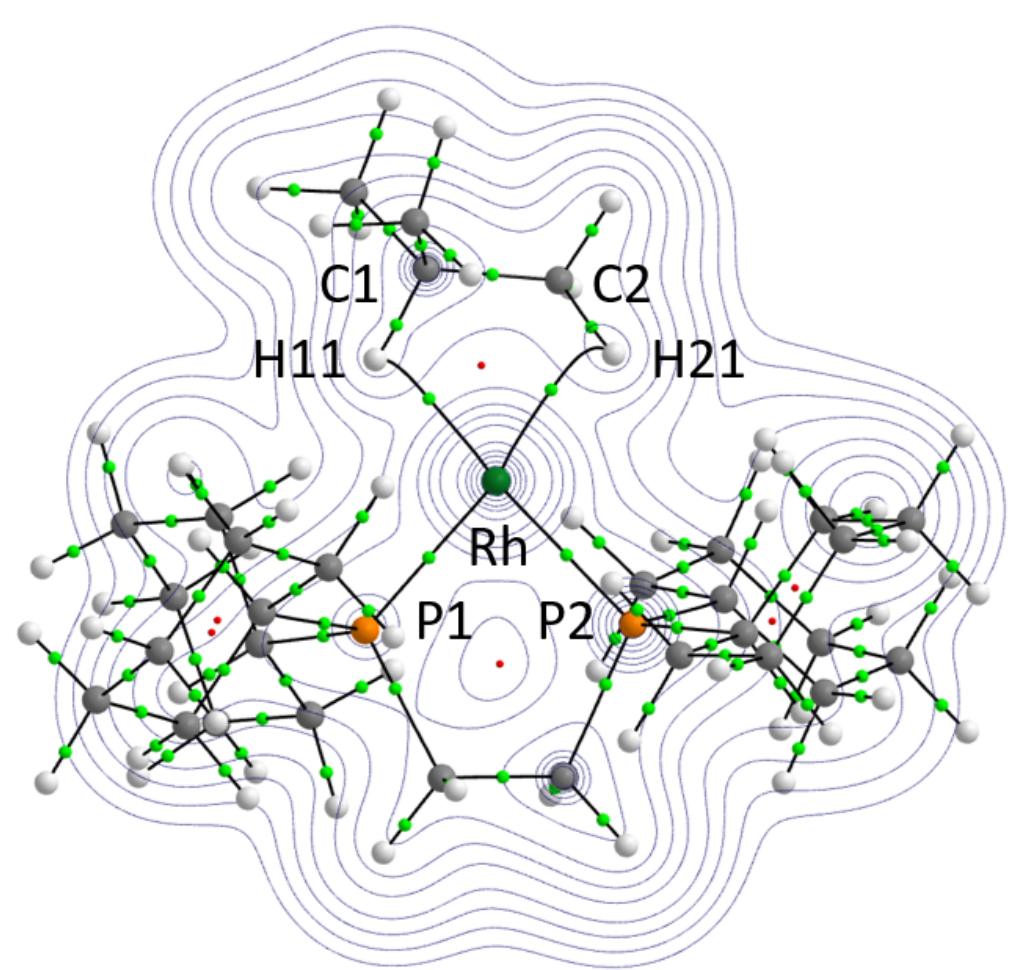

Figure S110: QTAIM molecular graph of [1-isobutane][BAr $\left.{ }_{4}\right]$ (fully optimised) with electron density contours in the H11-Rh-H21 plane.
Table S30: QTAIM BCP data and bond lengths for key bonds.

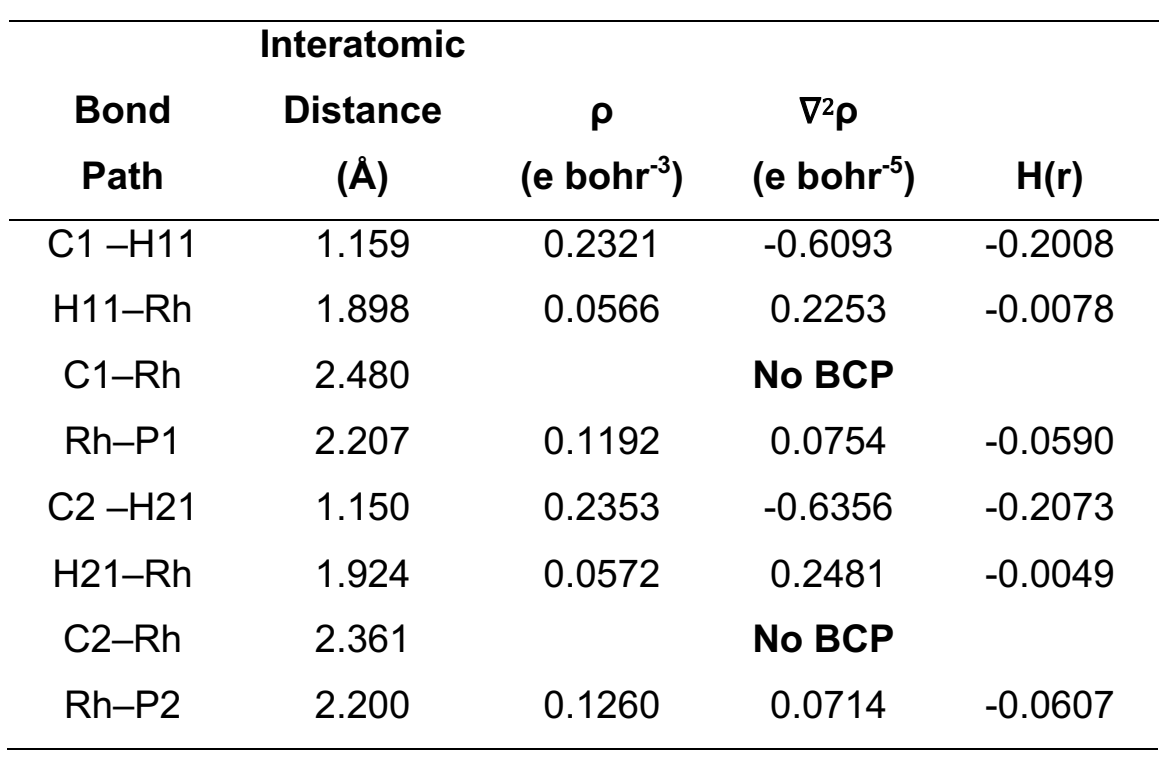




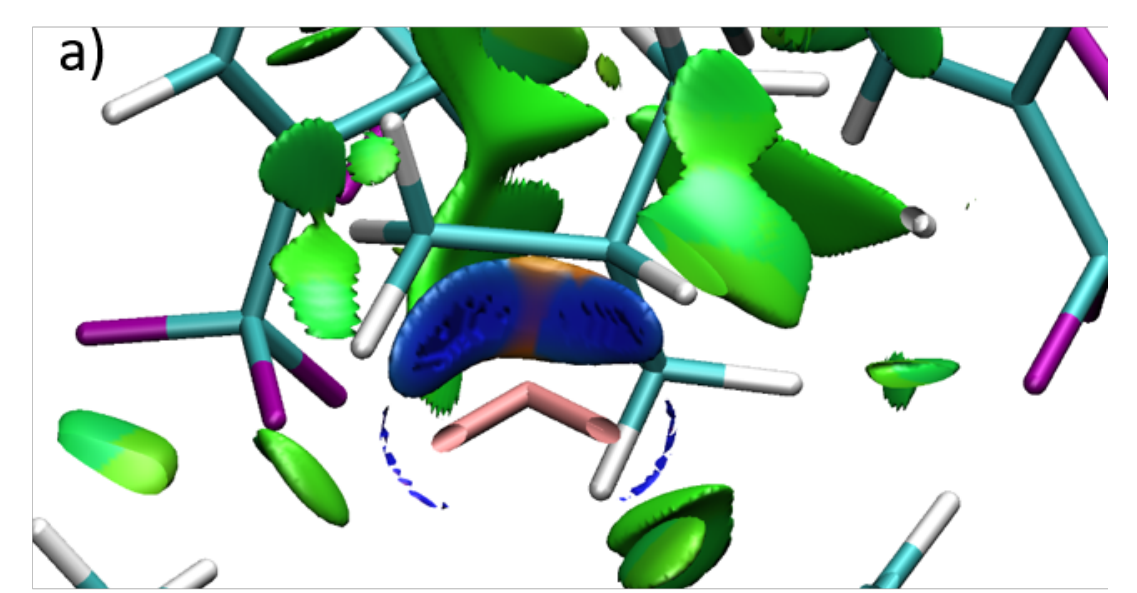

b)

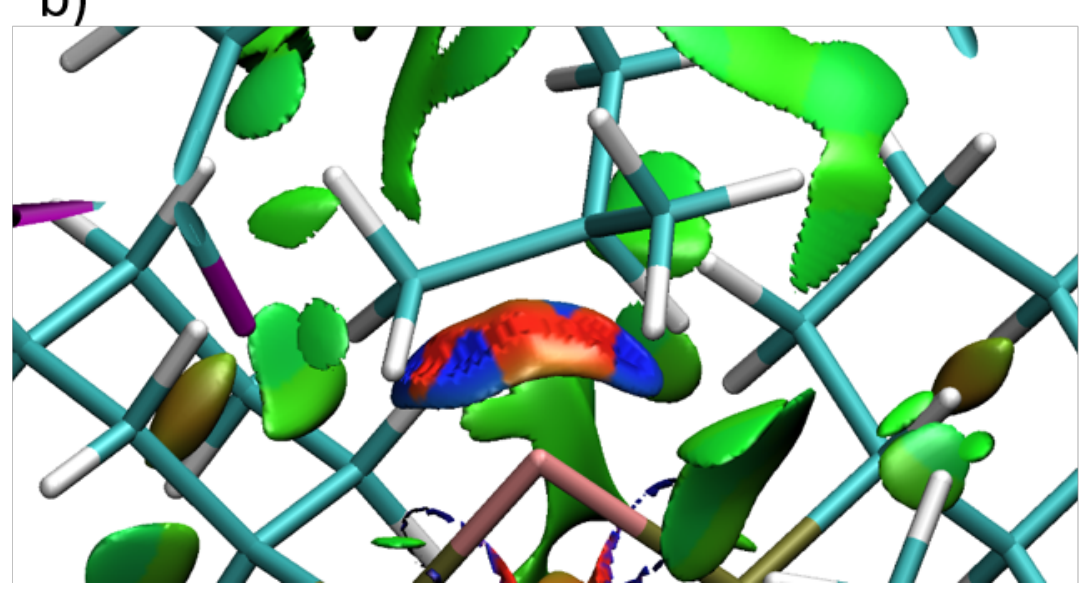

Figure S111: a) $\mathrm{NCl}$ Plot of [1-isobutane][BAr${ }_{4}$ ] (partially optimised) with isosurfaces generated for $s=0.3$ au and $-0.07<\rho<$ $0.07 \mathrm{au}$. View from Rh looking towards the alkane. b) View from above the alkane looking towards $\mathrm{Rh}$. a)
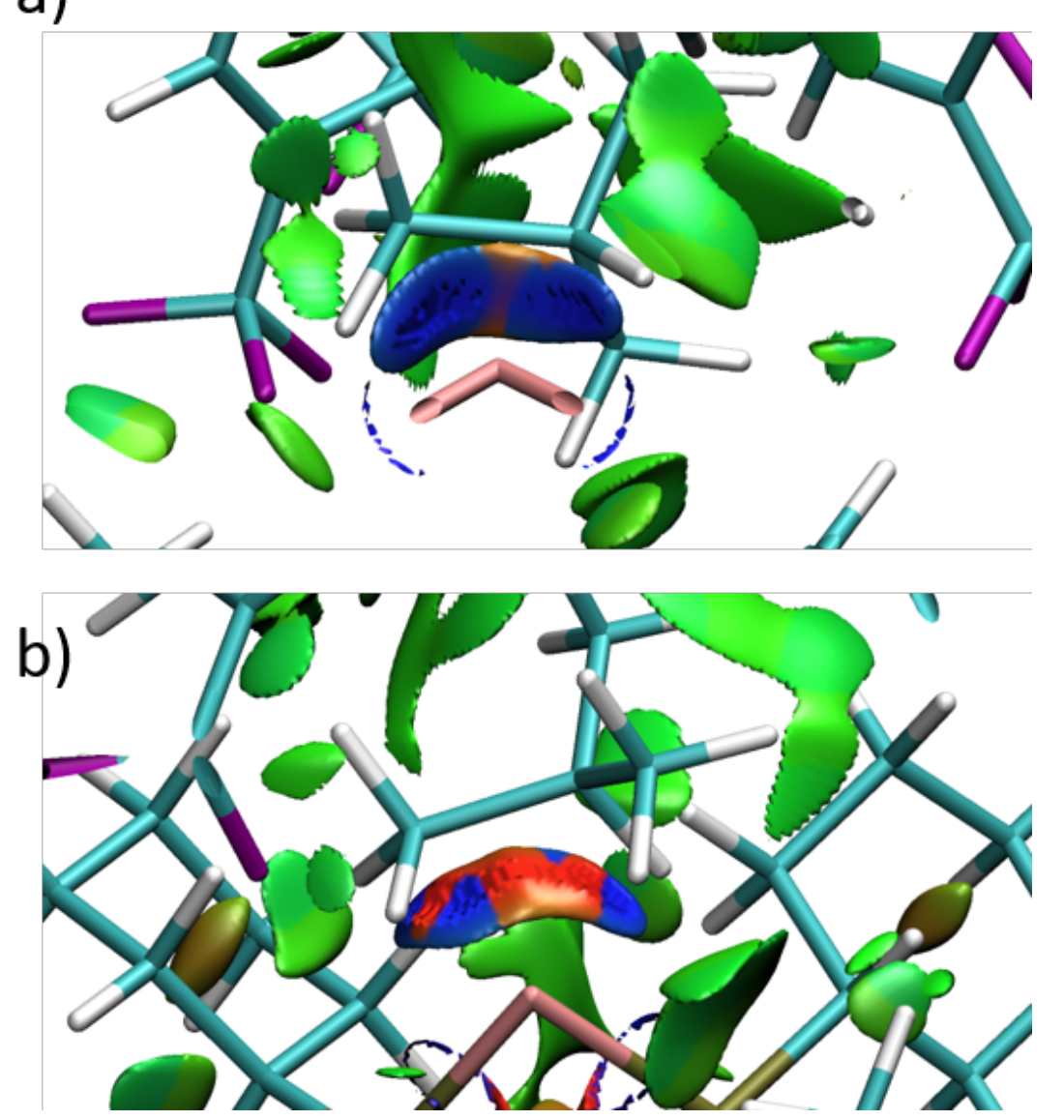

Figure S112: a) $\mathrm{NCl}$ Plot of [1-isobutane][BAr${ }_{4}$ ] (fully optimised) with isosurfaces generated for $\mathrm{s}=0.3$ au and $-0.07<\rho<0.07$ au. View from Rh looking towards the alkane. b) View from above the alkane looking towards Rh. 
Table s31: NBO data from [1-isobutane][BAr $\left.{ }_{4}\right]$ (partially optimised) showing the donating and accepting orbitals occupation and the $2^{\text {nd }}$ order perturbation theory interaction energy.

\begin{tabular}{|c|c|c|c|c|}
\hline $\begin{array}{c}\text { Donating } \\
\text { Orbital }\end{array}$ & Occupation & $\begin{array}{c}\text { Accepting } \\
\text { Orbital }\end{array}$ & Occupation & $\begin{array}{c}\text { Energy } \\
\text { (kcal/mol) }\end{array}$ \\
\hline \multicolumn{5}{|c|}{$\mathrm{C} 1-\mathrm{H} 11$} \\
\hline$\sigma(\mathrm{C}-\mathrm{H})$ & 1.86 & $\begin{array}{c}\sigma^{*}(\mathrm{Rh}- \\
\left.\mathrm{P}_{\text {trans }}\right)\end{array}$ & 0.28 & 21.41 \\
\hline$\sigma(\mathrm{C}-\mathrm{H})$ & 1.86 & $\begin{array}{c}\sigma^{*}(\mathrm{Rh}- \\
\left.\mathrm{P}_{\mathrm{Cis}}\right)\end{array}$ & 0.27 & 0.22 \\
\hline LP (Rh) & 1.97 & $\sigma^{*}(\mathrm{C}-\mathrm{H})$ & 0.03 & 0.05 \\
\hline LP (Rh) & 1.90 & $\sigma^{*}(\mathrm{C}-\mathrm{H})$ & 0.03 & 0.38 \\
\hline LP (Rh) & 1.87 & $\sigma^{*}(\mathrm{C}-\mathrm{H})$ & 0.03 & 3.40 \\
\hline$\sigma\left(\mathrm{Rh}-\mathrm{P}_{\text {cis }}\right)$ & 1.83 & $\sigma^{*}(\mathrm{C}-\mathrm{H})$ & 0.03 & 3.89 \\
\hline $\begin{array}{c}\sigma(\mathrm{Rh}- \\
\left.\mathrm{P}_{\text {trans }}\right)\end{array}$ & 1.83 & $\sigma^{*}(\mathrm{C}-\mathrm{H})$ & 0.03 & 1.63 \\
\hline \multicolumn{5}{|c|}{$\mathrm{C} 2-\mathrm{H} 21$} \\
\hline$\sigma(\mathrm{C}-\mathrm{H})$ & 1.89 & $\begin{array}{c}\sigma^{*}(\mathrm{Rh}- \\
\left.\mathrm{P}_{\text {trans }}\right)\end{array}$ & 0.27 & 22.30 \\
\hline$\sigma(\mathrm{C}-\mathrm{H})$ & 1.89 & $\begin{array}{c}\sigma^{*}(R h- \\
\left.P_{\text {Cis }}\right)\end{array}$ & 0.28 & 0.40 \\
\hline LP (Rh) & 1.95 & $\sigma^{*}(\mathrm{C}-\mathrm{H})$ & 0.05 & 0.44 \\
\hline LP (Rh) & 1.90 & $\sigma^{*}(\mathrm{C}-\mathrm{H})$ & 0.05 & 0.72 \\
\hline LP (Rh) & 1.87 & $\sigma^{*}(\mathrm{C}-\mathrm{H})$ & 0.05 & 3.80 \\
\hline$\sigma\left(\mathrm{Rh}-\mathrm{P}_{\text {cis }}\right)$ & 1.83 & $\sigma^{*}(\mathrm{C}-\mathrm{H})$ & 0.05 & 2.54 \\
\hline $\begin{array}{c}\sigma(\mathrm{Rh}- \\
\left.\mathrm{P}_{\text {trans }}\right)\end{array}$ & 1.83 & $\sigma^{*}(\mathrm{C}-\mathrm{H})$ & 0.05 & 0.69 \\
\hline
\end{tabular}

Table S32: NBO data from [1-isobutane][BAr ${ }_{4}$ ] (fully optimised) showing the donating and accepting orbitals occupation and the $2^{\text {nd }}$ order perturbation theory interaction energy.

\begin{tabular}{|c|c|c|c|c|}
\hline $\begin{array}{c}\text { Donating } \\
\text { Orbital }\end{array}$ & Occupation & $\begin{array}{c}\text { Accepting } \\
\text { Orbital }\end{array}$ & Occupation & $\begin{array}{c}\text { Energy } \\
\text { (kcal/mol) }\end{array}$ \\
\hline \multicolumn{5}{|c|}{$\mathrm{C} 1-\mathrm{H} 11$} \\
\hline$\sigma(\mathrm{C}-\mathrm{H})$ & 1.86 & $\begin{array}{c}\sigma^{*}(\mathrm{Rh}- \\
\left.\mathrm{P}_{\text {trans }}\right)\end{array}$ & 0.28 & 19.75 \\
\hline$\sigma(\mathrm{C}-\mathrm{H})$ & 1.86 & $\begin{array}{c}\sigma^{*}(\mathrm{Rh}- \\
\left.\mathrm{P}_{\mathrm{Cis}}\right)\end{array}$ & 0.27 & 0.17 \\
\hline LP (Rh) & 1.97 & $\sigma^{*}(\mathrm{C}-\mathrm{H})$ & 0.05 & 0.07 \\
\hline LP (Rh) & 1.90 & $\sigma^{*}(\mathrm{C}-\mathrm{H})$ & 0.05 & 0.29 \\
\hline LP (Rh) & 1.87 & $\sigma^{*}(\mathrm{C}-\mathrm{H})$ & 0.05 & 2.92 \\
\hline$\sigma\left(\mathrm{Rh}-\mathrm{P}_{\text {cis }}^{\prime}\right)$ & 1.83 & $\sigma^{*}(\mathrm{C}-\mathrm{H})$ & 0.05 & 3.70 \\
\hline $\begin{array}{c}\sigma(\mathrm{Rh}- \\
\left.\mathrm{P}_{\text {trans }}\right)\end{array}$ & 1.83 & $\sigma^{*}(\mathrm{C}-\mathrm{H})$ & 0.05 & 1.69 \\
\hline \multicolumn{5}{|c|}{$\mathrm{C} 2-\mathrm{H} 21$} \\
\hline$\sigma(\mathrm{C}-\mathrm{H})$ & 1.88 & $\begin{array}{c}\sigma^{*}(\mathrm{Rh}- \\
\left.\mathrm{P}_{\text {trans }}\right)\end{array}$ & 0.27 & 22.55 \\
\hline$\sigma(\mathrm{C}-\mathrm{H})$ & 1.88 & $\begin{array}{c}\sigma^{*}(R h- \\
\left.P_{C i s}\right)\end{array}$ & 0.28 & 0.43 \\
\hline LP (Rh) & 1.95 & $\sigma^{*}(\mathrm{C}-\mathrm{H})$ & 0.05 & 0.67 \\
\hline LP (Rh) & 1.90 & $\sigma^{*}(\mathrm{C}-\mathrm{H})$ & 0.05 & 1.08 \\
\hline LP (Rh) & 1.87 & $\sigma^{*}(\mathrm{C}-\mathrm{H})$ & 0.05 & 3.69 \\
\hline$\sigma\left(\mathrm{Rh}-\mathrm{P}_{\text {cis }}^{\prime}\right)$ & 1.83 & $\sigma^{*}(\mathrm{C}-\mathrm{H})$ & 0.05 & 2.34 \\
\hline $\begin{array}{c}\sigma(\mathrm{Rh}- \\
\left.\mathrm{P}_{\text {trans }}\right)\end{array}$ & 1.83 & $\sigma^{*}(\mathrm{C}-\mathrm{H})$ & 0.05 & 0.70 \\
\hline
\end{tabular}




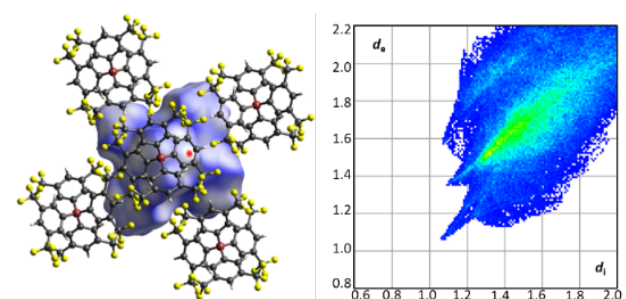

Full System

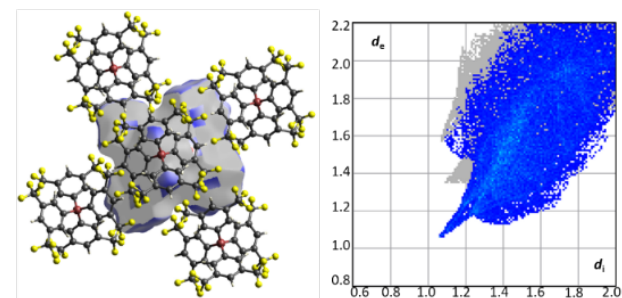

H...H $(24.9 \%)$

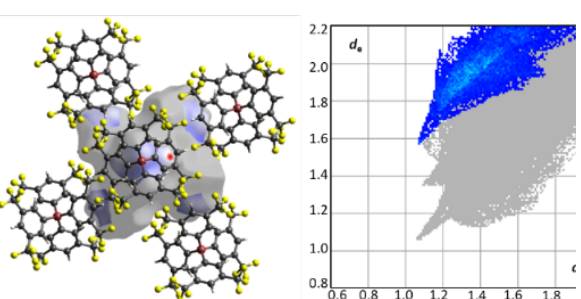

$H \cdots C(12.5 \%)$

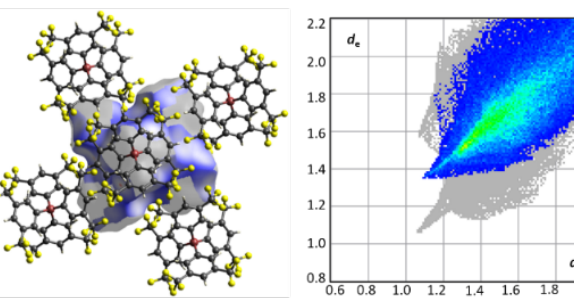

$H \cdots F(62.6 \%)$

Figure S113: Hirshfeld Surfaces of [1-isobutane][BAr${ }_{4}$ ] (partially optimised) showing normalised distance and associated fingerprint plots. Percentage of each type of contact is shown in brackets.

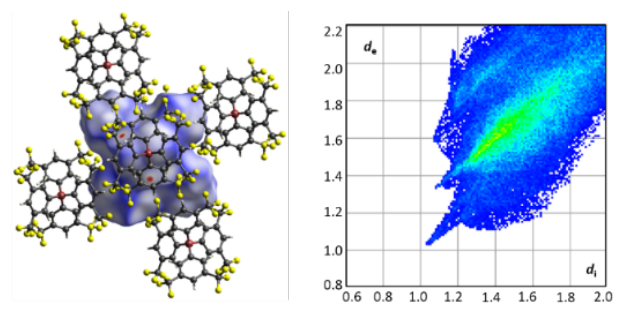

Full System

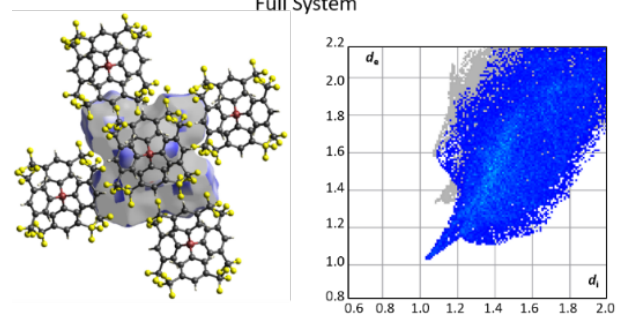

H...H $(25.0 \%)$

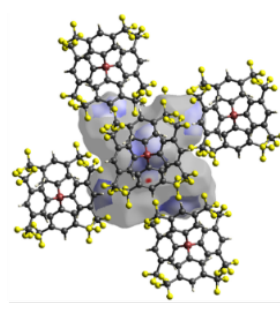

$\mathrm{H} \cdots \mathrm{C}(12.6 \%)$
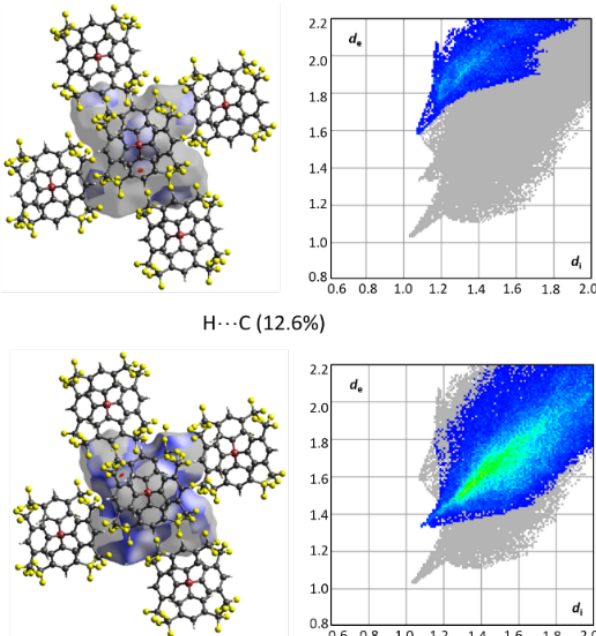

$H \cdots F(64.4 \%)$

Figure S114: Hirshfeld Surfaces of [1-isobutane][BAr ${ }_{4}$ ] (fully optimised) showing normalised distance and associated fingerprint plots. Percentage of each type of contact is shown in brackets. 


\section{S.6. References}

S1 Chadwick, F. M.; Rees, N. H.; Weller, A. S.; Krämer, T.; lannuzzi, M.; Macgregor, S. A. A RhodiumPentane Sigma-Alkane Complex: Characterization in the Solid State by Experimental and Computational Techniques. Angew. Chem. Int. Ed. 2016, 55 (11), 3677-3681.

S2 Pike, S. D.; Chadwick, F. M.; Rees, N. H.; Scott, M. P.; Weller, A. S.; Krämer, T.; Macgregor, S. A. SolidState Synthesis and Characterization of $\sigma$-Alkane Complexes, [Rh(L2) $(\mathrm{H} 2, \mathrm{H} 2-\mathrm{C} 7 \mathrm{H} 12)][\mathrm{BArF} 4](\mathrm{L} 2=$ Bidentate Chelating Phosphine). J. Am. Chem. Soc. 2015, 137 (2), 820-833.

S3 Earl, W. L.; Vanderhart, D. L. Measurement Of13C Chemical Shifts in Solids. J. Magn. Reson. 1982, 48 (1), 35-54.

S4 Lubben, A. T.; Scott Mclndoe, J.; Weller, A. S. Coupling an Electrospray lonwization Mass Spectrometer with a Glovebox: A Straightforward, Powerful, and Convenient Combination for Analysis of Air-Sensitive Organometallics. Organometallics 2008, 27 (13), 3303-3306.

S5 Chadwick, F. M.; McKay, A. I.; Martinez-Martinez, A. J.; Rees, N. H.; Krämer, T.; Macgregor, S. A.; Weller, A. S. Solid-State Molecular Organometallic Chemistry. Single-Crystal to Single-Crystal Reactivity and Catalysis with Light Hydrocarbon Substrates. Chem. Sci. 2017, 8 (9), 6014-6029.

S6 Nowell, H.; Barnett, S. A.; Christensen, K. E.; Teat, S. J.; Allan, D. R. I19, the Small-Molecule SingleCrystal Diffraction Beamline at Diamond Light Source. J. Synchrotron Radiat. 2012, 19 (3), 435-441.

S7 McKay, A. I.; J. Bukvic, A.; E. Tegner, B.; L. Burnage, A.; J. Martínez-Martínez, A.; H. Rees, N.; A. Macgregor, S.; S. Weller, A. Room Temperature Acceptorless Alkane Dehydrogenation from Molecular б-Alkane Complexes. J. Am. Chem. Soc. 2019, 141 (29), 11700-11712.

S8 Benedict, J. B.; Coppens, P. Kinetics of the Single-Crystal to Single-Crystal Two-Photon

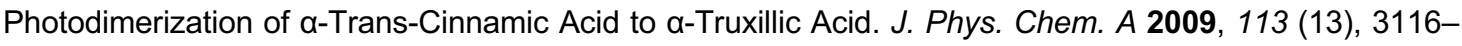
3120.

S9 Hasegawa, K.; Asami, R.; Takahashi, K. 13C NMR Chemcial Shifts and Cationic Reactvity of Linear Conjugated Dienes. Bull. Chem. Soc. Jpn. 1978, 51 (3), 916-920.

S10 (E)-3-methyl-1,3-pentadiene -- AIST: Spectral Database for Organic Compounds -- (SDBS No.: 293) -Date Acc. 25-06-2020 https://sdbs.db.aist.go.jp/sdbs/cgi-bin/landingpage?sdbsno=293 (accessed Jun 25, 2020).

S11 (Z)-3-methyl-1,3-pentadiene -- AIST: Spectral Database for Organic Compounds -- (SDBS No.: 292) -Date Acc. 25-06-2020 https://sdbs.db.aist.go.jp/sdbs/cgi-bin/landingpage?sdbsno=292 (accessed Jun 25, 2020).

S12 2-Ethyl-1-butene -- AIST: Spectral Database for Organic Compounds -- (SDBS No.: 10346) -- Date Acc. 03-06-2020 https://sdbs.db.aist.go.jp/sdbs/cgi-bin/landingpage?sdbsno=10346 (accessed Jun 3, 2020).

S13 3-Methyl-2-pentene, mixture of cis and trans -- Sigma-Aldrich -- (Cat. Number 111775) - Date Acc. 03-07$2020 \mathrm{https} / / /$ www.sigmaaldrich.com/catalog/product/aldrich/111775?lang=en\&region=GB (accessed Jul $3,2020)$.

S14 3-Methyl-1-pentene -- Sigma-Aldrich -- (Cat. Number 111147) -- - Date Acc. 03-07-2020 https://www.sigmaaldrich.com/catalog/product/aldrich/111147?lang=en\&region=GB (accessed Jul 3, 2020).

S15 Oxford Diffraction Ltd.; 2011

S16 Sheldrick, G. M. A Short History of SHELX. Acta Crystallogr. Sect. A Found. Crystallogr. 2008, 64 (1), 112-122.

S17 Sheldrick, G. M. SHELXT - Integrated Space-Group and Crystal-Structure Determination. Acta 
Crystallogr. Sect. A Found. Crystallogr. 2015, 71 (1), 3-8.

S18 Dolomanov, O. V.; Bourhis, L. J.; Gildea, R. J.; Howard, J. A. K.; Puschmann, H. OLEX2: A Complete Structure Solution, Refinement and Analysis Program. J. Appl. Crystallogr. 2009, 42 (2), 339-341.

S19 Cosier, B. J.; Glazer, A. M. A Nitrogen-Gas-Stream Cryostat for General X-Ray Diffraction Studies. J. Appl. Crystallogr. 1986, 19 (2), 105-107.

S20 Pike, S. D.; Thompson, A. L.; Algarra, A. G.; Apperley, D. C.; Macgregor, S. A.; Weller, A. S. Synthesis and Characterization of a Rhodium(I) $\sigma$-Alkane Complex in the Solid State. Science 2012, 337, 1648.

S21 McKay, A. I.; Krämer, T.; Rees, N. H.; Thompson, A. L.; Christensen, K. E.; Macgregor, S. A.; Weller, A. S. Formation of a $\sigma$-Alkane Complex and a Molecular Rearrangement in the Solid-State: [Rh(Cyp 2 PCH

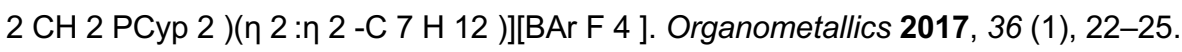

McKay, A. I.; Martínez-Martínez, A. J.; Griffiths, H. J.; Rees, N. H.; Waters, J. B.; Weller, A. S.; Krämer, T.; MacGregor, S. A. Controlling Structure and Reactivity in Cationic Solid-State Molecular Organometallic Systems Using Anion Templating. Organometallics 2018, 37 (20), 3524-3532.

Martínez-Martínez, A. J.; Tegner, B. E.; McKay, A. I.; Bukvic, A. J.; Rees, N. H.; Tizzard, G. J.; Coles, S. J.; Warren, M. R.; Macgregor, S. A.; Weller, A. S. Modulation of $\sigma$-Alkane Interactions in [Rh(L2)(Alkane)]+ Solid-State Molecular Organometallic (SMOM) Systems by Variation of the Chelating Phosphine and Alkane: Access to H2,H2- - -Alkane Rh(I), H1- $\sigma$-Alkane Rh(III) Complexes, and Alkane Encapsulation. J. Am. Chem. Soc. 2018, 140 (44), 14958-14970.

Van de Vondele, J.; Krack, M.; Mohamed, F.; Parrinello, M.; Chassaing, T.; Hutter, J. Comput. Phys. Commun. 2005, 167, 103-128. Hutter, J.; Iannuzzi, M.; Schiffmann, F.; VandeVondele, J. Wires Comput. Mol. Sci. 2014, 4, 15. VandeVondele, J.; Hutter, J. J. Chem. Phys. 2007, 127, 114105.

S27 Hartwigsen, C.; Goedecker, S.; Hutter, J. Phys. Rev. B 1998, 58, 3641.

S28 Goedecker, S.; Teter, M.; Hutter, J. Phys. Rev. B 1996, 54, 1703.

S29 Krack, M. Theor. Chem. Acc. 2005, 114, 145.

S30 Perdew, J. P.; Burke, K.; Ernzerhof, M. Phys. Rev. Lett. 1996, 77, 3865.

S31 Grimme, S.; Antony, J.; Ehrlich, S.; Krieg, H. J. Chem. Phys. 2010, 132, 154104.

S32 Mercury CSD 2.0, Macrae, C. F.; Bruno, I. J.; Chisholm, J. A.; Edgington, P. R.; McCabe, P.; Pidcock, E.; Rodriguez-Monge, L.; Taylor, R.; van de Streek, J.; Wood, P. A. J. Appl. Cryst., 2008, 41, 466-470.

S33 Ghysels, A.; Verstraelen, T.; Hemelsoet, K.; Waroquier, M.; Van Speybroeck, V. J. Chem. Inf. Model. 2010, 50, 1736-1750.

S34 VESTA 3, K. Momma and F. Izumi, J. Appl. Cryst. 2011, 44, 1272-1276.

S35 CrystalExplorer 17, M. J. Turner, J. J. McKinnon, S. K. Wolff, D. J. Grimwood, P. R. Spackman, D. Jayatilaka and M. A. Spackman, 2017, University of Western Australia.

S36 Gaussian 09 (Revision D.01); Frisch, M. J.; Trucks, G. W.; Schlegel, H. B.; Scuseria, G. E.; Robb, M. A.; Cheeseman, J. R.; Scalmani, G.; Barone, V.; Mennucci, B.; Petersson, G. A.; Nakatsuji, H.; Caricato, M.; Li, X.; Hratchian, H. P.; Izmaylov, A. F.; Bloino, J.; Zheng, G.; Sonnenberg, J. L.; Hada, M.; Ehara, M.; Toyota, K.; Fukuda, R.; Hasegawa, J.; Ishida, M.; Nakajima, T.; Honda, Y.; Kitao, O.; Nakai, H.; Vreven, T.; J. A. Montgomery, J.; Peralta, J. E.; Ogliaro, F.; Bearpark, M.; Heyd, J. J.; Brothers, E.; Kudin, K. N.; Staroverov, V. N.; Keith, T.; Kobayashi, R.; Normand, J.; Raghavachari, K.; Rendell, A.; Burant, J. C.; lyengar, S. S.; Tomasi, J.; Cossi, M.; Rega, N.; Millam, J. M.; Klene, M.; Knox, J. E.; Cross, J. B.; Bakken, V.; Adamo, C.; Jaramillo, J.; Gomperts, R.; Stratmann, R. E.; Yazyev, O.; Austin, A. J.; Cammi, R.; Pomelli, C.; Ochterski, J. W.; Martin, R. L.; Morokuma, K.; Zakrzewski, V. G.; Voth, G. A.; Salvador, P.; Dannenberg, J. J.; Dapprich, S.; Daniels, A. D.; Farkas, O.; Foresman, J. B.; Ortiz, J. V.; Cioslowski, J.; Fox, D. J.; Gaussian Inc., Wallingford, CT, 2013. 
S37 Becke, A. D. Phys. Rev. A 1988, 38, 3098.

S38 Perdew, J. P. Phys. Rev. B 1986, 33, 8822.

S39 Andrae, D.; Haussermann, U.; Dolg, M.; Stoll, H.; Preuss, H. Theor. Chim. Acta 1990, 77, 123.

S40 Hollwarth, A.; Bohme, M.; Dapprich, S.; Ehlers, A. W.; Gobbi, A.; Jonas, V.; Kohler, K. F.; Stegmann, R.; Veldkamp, A.; Frenking, G. Chem. Phys. Lett. 1993, 208, 237.

S41 Hehre, W. J.; Ditchfield, R.; Pople, J. A. J. Chem. Phys. 1972, 56, 2257.

S42 Hariharan, P. C.; Pople, J. A. Theor. Chim. Acta 1973, 28, 213.

S43 Bader, R. F. W. Atoms in Molecules: A Quantum Theory; Oxford University Press, 1990.

S44 AIMAll (Version 17.11.14), Todd A. Keith, TK Gristmill Software, Overland Park KS, USA, 2017 (aim.tkgristmill.com)

S45 NBO 6.0. E. D. Glendening, J. K. Badenhoop, A. E. Reed, J. E. Carpenter, J. A. Bohmann, C. M. Morales, C. R. Landis, and F. Weinhold (Theoretical Chemistry Institute, University of Wisconsin, Madison, WI, 2013); http://nbo6.chem.wisc.edu/

S46 E. R. Johnson, S. Keinan, P. Mori-Sanchez, J. Contreras-Garcia, A. J. Cohen, and W. Yang, J. Am. Chem. Soc., 2010, 132, 6498-6506.

S47 J. Contreras-Garcia, E. R. Johnson, S. Keinan, R. Chaudret, J-P. Piquemal, D. N. Beratan, and W. Yang. J. Chem. Theory Comput. 2011, 7, 625-632.

S48 Chemcraft - graphical software for visualization of quantum chemistry computations. https://www.chemcraftprog.com 